\title{
Development of a non-invasive ocular drug delivery device
}


ISBN: 978-94-6419-209-4

Printing: Gildeprint, Enschede Cover design and layout: Christian J.F. Bertens

Chapter 1, Figure 1-1 and 1-2: Rogier Trompert Medical Art

\section{(C) 2021 Christian J.F. Bertens}

All rights reserved. No part of this thesis may be reproduced or transmitted in any form or by any means, electronic, or mechanical, including photocopying, recording or any information storage or retrieval system, without permission in writing from the author, or when appropriate, from the publishers of the publication. 


\title{
Development of a non-invasive ocular drug delivery device
}

\author{
Proefschrift
}

ter verkrijging van de graad van doctor aan de Universiteit Maastricht, op gezag van de Rector Magnificus, prof. dr. Rianne M. Letschert volgens het besluit van het College van Decanen,

in het openbaar te verdedigen

op maandag 7 juni 2021 om 13.00 uur

door

Christian Johannes Franciscus Bertens

Geboren 4 april 1990 te Udenhout 


\section{Promotor}

Prof. dr. Rudy M.M.A. Nuijts

\section{Copromotoren}

Dr. Marlies Gijs

Dr. Frank J.H.M. van den Biggelaar

\section{Beoordelingscommissie}

Prof. dr. Jos H.H.J. Prickaerts, voorzitter

Prof. dr. Harald H.W. Schmidt

Prof. dr. Christine Jérôme (University of Liège, Liège, Belgium)

Dr. Jens C. Thies (Royal DSM, Heerlen, the Netherlands) 


\section{Contents}

Chapter 1. General introduction 1

$\begin{array}{lll}\text { Chapter 2. Topical drug delivery devices: a review } & 17\end{array}$

Chapter 3. Design of the ocular coil, a new device for non-invasive 45 drug delivery

Chapter 4. Pharmacokinetics and efficacy of a ketorolac-loaded ocular coil in New Zealand White rabbits

Chapter 5. Safety and comfort of an innovative drug delivery device in healthy subjects

Chapter 6. Confocal Raman spectroscopy: evaluation of a noninvasive technique for the detection of topically applied ketorolac tromethamine in vitro and in vivo

Chapter 6a. Pipeline for the removal of hardware related artifacts 141 and background noise for Raman spectroscopy

Chapter 6b. In vitro and in vivo datasets of topically applied ketorolac 155 tromethamine in aqueous humor using Raman spectroscopy

Chapter 7. Validation of computerized quantification of ocular 167 redness

Chapter 8. General discussion

Chapter 9. Impact

Summary

Samenvatting

Dankwoord / Acknowledgements

Appendix I. Curriculum Vitae

Appendix II. List of publications 


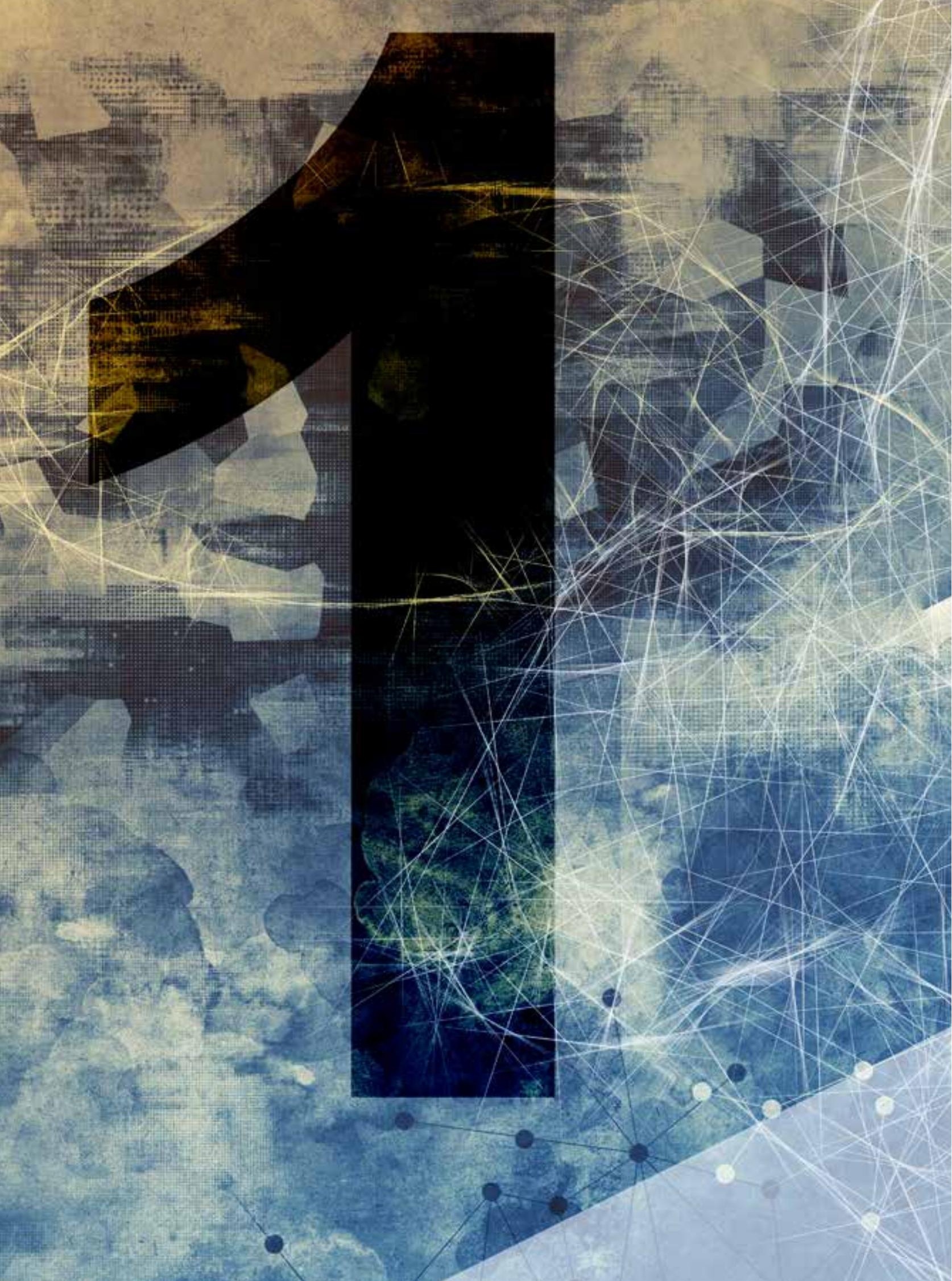




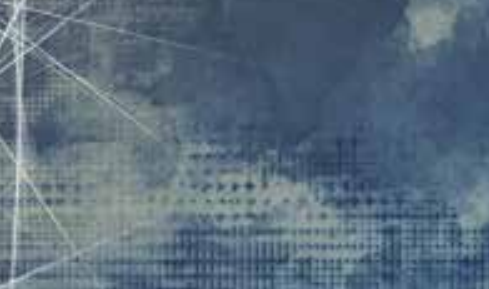

cha[pter 1.

\section{General introduction -}

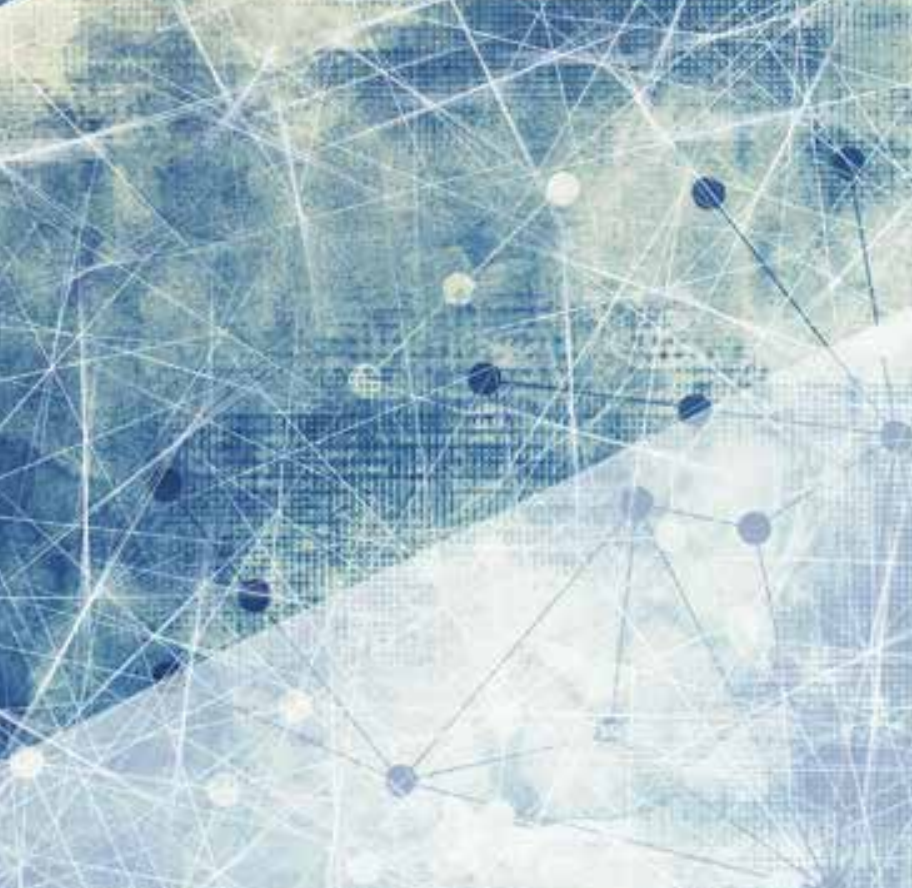

Christian J.F. Bertens 


\subsection{Anatomy of the eye}

The eye is the organ of vision and consists of the eyeball (bulbus oculi) and the optic nerve. The bulbus is located anterior to the orbita and is supported by several adnexa (e.g., eyelids, conjunctiva, lacrimal system, and muscles).

The bulbus measures approximately 23 to $25 \mathrm{~mm}$ in anteroposterior diameter (axial length) [1] and has a spherical arrangement. In the orbita, the bulbus is supported by connective tissue (the fascial sheath) consisting of the bulbar facia (tenon capsule, which forms the socket for the bulbus), and bulbar conjunctiva (mucous membrane covering the bulbus and part of the surface of the eyelids). A loose layer of connective tissue, the episcleral space, lies between the fascial sheath and the outer layer of the bulbus. The eyeball layers consist of a fibrous outer coat (the sclera) and an inner coat (retina).[2] A vascularized layer (the uvea), consisting of the choroid, ciliary body, and iris, covers the inner-sclera.

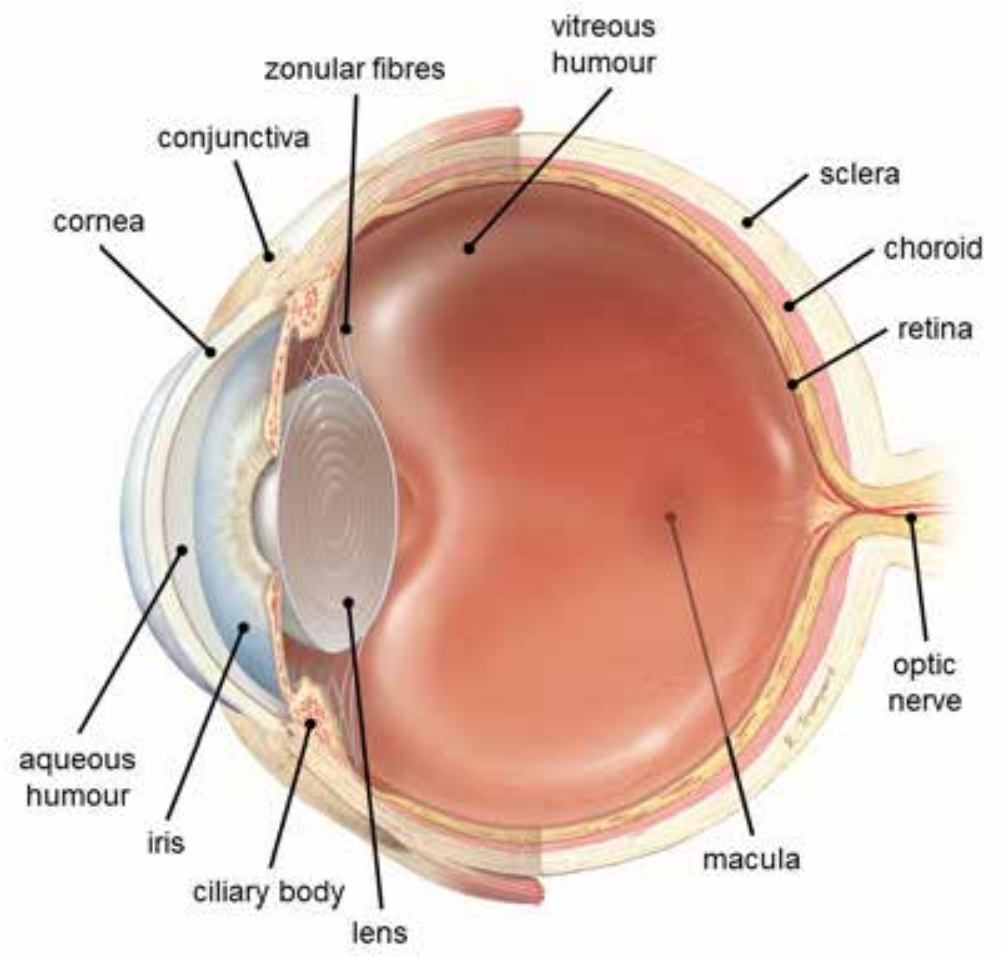

Figure 1-1. Anatomy of the eye. Both the anterior chamber (located between the cornea and the iris) and the posterior chamber (located between the iris and the lens) are filled with aqueous humour. The vitreous cavity lies posterior to the lens and is filled with vitreous humour. The bulbus is formed by the sclera and forms the vitreous cavity. The inside of the vitreous cavity is covered by the retina. The most sensitive area of the retina is named the macula. Between the retinal layers and the sclera (the outside layer of the eye) a highly vascularized layer is located, the choroid. 
From anterior to posterior, the structures of the bulbus are the cornea, anterior chamber, iris, posterior chamber, lens, vitreous chamber, retina, choroid, and sclera (figure 1-1). Furthermore, the bulbus can also be divided in three compartments, i.e. the anterior chamber (between the cornea and iris), the posterior chamber (between the iris and the lens), and the vitreous cavity (behind the lens). The anterior and posterior chamber are connected through the pupil an both filled with aqueous humour (about $200 \mu \mathrm{L}$ for the anterior chamber and about $60 \mu \mathrm{L}$ for the posterior chamber), whereas the vitreous chamber is filled with vitreous humour (about 5 - 6 $\mathrm{mL}) \cdot[1]$

Aqueous humour is produced by the ciliary body and flows via the posterior chamber through the pupil into the anterior chamber. In the anterior chamber, aqueous humour is cleared via the canal of Schlemm ( $90 \%$ of clearance), located in the trabecular meshwork, or via the uveoscleral pathway (10\% clearance) (figure 1-2). From Schlemm's canal, the aqueous humour is transported into systemic circulation by the aqueous vein.[3,4] Aqueous humour provides nutrients to the anterior segment of the eye, removes metabolic waste products, and regulates the intraocular pressure (IOP).[1,4] Vitreous humour is a transparent gel composed of water, collagen, and hyaluronan.[5] It fills the vitreous chamber and, therefore, helps to maintain the eye's spherical shape and transports nutrients and solutes to, and from, the lens.[1]

\subsubsection{The anterior segment of the bulbus}

Anteriorly, the bulbus is covered by clear tissue, i.e. the cornea. The cornea has a stronger curvature (radius of $8 \mathrm{~mm}$ ) compared to the sclera (radius of $12 \mathrm{~mm}$ ).[6] Because of this stronger curvature, the cornea is responsible for about two thirds of the eye's refractive power (approximately +42 diopters). [6] The cornea consists of five layers, i.e. from anterior to posterior: epithelium, Bowman's layer, stroma, Descemet's membrane, and endothelium (figure 1-2). The cornea is completely avascular and receives its nutrition from peripheral capillary beds, internally from the aqueous humour, and externally from lacrimal fluids. The lacrimal fluid also provides oxygen absorbed from the air to the cornea.[2]

Posterior to the anterior segment lies the iris and the lens. The iris consists of the dilator muscle, sphincter muscle and pigmented epithelium. The muscles of the iris are capable to adapt the pupil size in order to regulate the amount of light on the retina.[3] The lens is a transparent structure of crystalline proteins and is responsible for the refraction and transmission of light to the retina. A collagen type IV membrane, called lens capsule, encapsulates the entire lens. The anterior lens surface is lined by a single layer of lens epithelial cells. The nucleus of the lens is prenatally formed from embryonic and foetal lens nuclei, whereas the lens cortex 
continuously grows from proliferating lens epithelial cells that mature into lens fibres. Therefore, the lens is the only tissue in the body that never stops growing.[7]

The lens is attached to the ciliary body that helps to change the shape of the lens in order to bring an image into focus on the retina. When the muscle of the ciliary body contracts, the zonular fibres relax resulting in less tension on the lens capsule and the lens becomes more convex. This mechanism is called accommodation.[8]

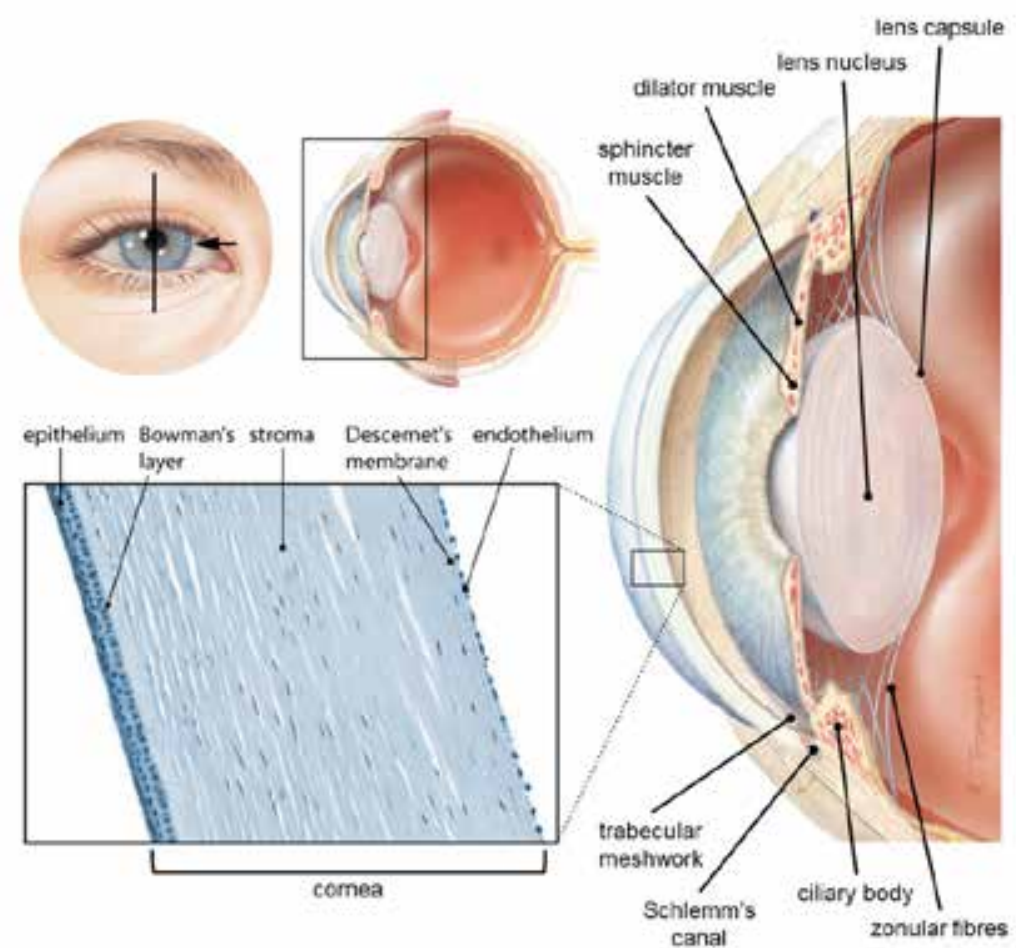

Figure 1-2. Anatomy of the anterior segment of the eye. The inset shows a sagittal section of the layers of the cornea: epithelium, Bowman's layer, stroma, Descemet's membrane, and endothelium.

\subsubsection{The posterior segment of the bulbus}

The inner part of the vitreous cavity is covered by the retina that converts light into neural signals and sends these signals to the brain for visual recognition. The central area of the retina is the macula. It measures about $5.5 \mathrm{~mm}$ in diameter.[5] The central $1.5 \mathrm{~mm}$ of the macula is the fovea that is specialised in high spatial acuity (the ability to resolve fine details) and colour vision. The highly structured retina contains multiple layers. Light must travel through the full thickness of the retina to reach the photoreceptors (rods and cones). When light reaches the phototoreceptors, photoreceptive pigment in the cells is modified (phototransduction) and a signal is 
transmitted to the brain. The brain translates the signal and forms a representation of the observed. [5]

Rods and cones rest on the retinal pigment epithelium (RPE), a monolayer of pigmented cells that are connected via the Bruch's membrane to the choroid. Blood enters the choroid via the ciliary arteries and is transported back into systemic circulation by the vortex veins. The choroid is covered by the sclera, the protective opaque outer layer of the eye ('white of the eye'). The sclera is composed of irregularly arranged lamellae of collagen fibrils interspersed with proteoglycans and non-collagenous glycoproteins.[5]

\subsubsection{The conjunctiva and tear film}

The bulbus is protected by its location within the orbita, but also by the eyelids, and tear film. A thin mucous membrane, i.e. the conjunctiva, connects the bulbus to the eyelids. The conjunctiva is connected to the bulbus at the limbus (transition from cornea to sclera) and runs to the tip of the eyelids (mucocutaneous junction).[3] The conjunctival layer on the bulbus is called the bulbar conjunctiva. This forms a small pocket, cul de sac or conjunctival fornix, and continues on the posterior part of the eyelids, i.e. the palpebral conjunctiva.[1,3] The conjunctiva holds numerous goblet cells and a thin, richly vascularized substantia propria containing lymphatic vessels, plasma cells, macrophages, and mast cells.[1]

The tear film helps to remove debris from the eye, lubricates the cornea-eyelid interface and protects the eye from dehydration. The tear film consists of three layers: an outer lipid layer, a middle aqueous layer and an inner mucin layer. The lipid layer is produced by the meibomian glands in the upper and lower eyelids and prevents the tear film from evaporating. The aqueous layer is produced by the accessory lacrimal glands located in the conjunctival fornix and consists of electrolytes, water, and proteins. It supplies oxygen to the cornea and provides an anti-bacterial and anti-viral defence. The mucus layer is produced by the conjunctival goblet-cells and converts the corneal epithelium from a hydrophobic layer into a hydrophilic layer and lowers the surface tension to stabilize the tear film.[1]

\subsection{Topical drug delivery}

Most ocular conditions are treated with topically applied drugs, such as eye drops or ointments. To be effective, drugs must penetrate across the eye's tissue barriers (e.g., cornea, sclera, and conjunctiva).[9] There are two main topical drug delivery routes. First, in the corneal delivery route, drug penetrates through the cornea into the anterior chamber and is cleared (mainly) via the trabecular meshwork.[10-14] Second, in the non-corneal drug delivery route, drugs penetrate via the conjunctiva through the sclera into the choroid, and to some extent, through the retina into the 
vitreous cavity.[15-18] In the vitreous cavity, small drug molecules are cleared via the blood-retina barrier. However, most of the drug will reach the anterior chamber via aqueous humour flow, and are cleared via the trabecular meshwork.[14,19,20]

\subsubsection{Eye drops}

Eye drops are the most commonly prescribed form of topical drugs for the treatment and prevention of ocular diseases.[21] Despite the high prescription rate of eye drops and the achieved therapeutic concentrations in anterior segment tissues, eye drops have significant disadvantages. Besides systemic side effects [22] and local toxicity $[23,24]$, the main disadvantages of eye drops are low drug bioavailability $[19,25,26]$ and poor patient compliance (the degree to which a patient correctly follows medical advice).[27-29]

Low bioavailability of the drug is caused by pre-corneal loss of the drug (by systemic conjunctival elimination, blinking, induced lacrimation, the tear film and rapid tear turnover).[11,12,30-32] Typically, less than $5 \%$ of the total administered dose reaches its target destination in the anterior chamber.[11,31,32]

In order to maintain effective concentrations, ocular drugs need to be administered frequently resulting in poor patient compliance.[33-35] Non-compliance compromises the effectiveness of the prescribed therapy.[27,29] Frequently reported reasons for non-compliance include forgetfulness $(26.7 \%$ of patients treated with eye drops), limited access to eye drops (20\%), and insufficient ability to properly selfinstil the eye drops (16.2\%).[27] Because of these reasons, formal or informal care is often required, which increases healthcare costs. Not only treatments for chronic ocular conditions (e.g., glaucoma [36,37]) encounter these problems, also postoperative treatments (e.g., after cataract surgery [28]) are affected by high noncompliance.

\subsection{The need for innovation}

To encounter the low drug bioavailability and high non-compliance, a new method for topical drug delivery is needed. This new method needs to be comfortable, should not interfere with vision, and have the capacity to deliver drugs over a prolonged period of time in order to remove (or lower) the number of application moments. Besides a new method for drug delivery, there is a need for objective evaluation methods for efficacy and safety of drug delivery devices.

\subsubsection{Drug delivery device}

In this research project, an ocular coil has been developed. The ocular coil consists of a coiled and coated surgical steel wire, filled with a drug eluting matrix in the innerlumen, and is capped on both extremities with a dome-shaped UV-curable acrylate 
urethane cap to soften its extremities and to prevent the matrix from escaping. The ocular coil is designed for insertion in the inferior ocular conjunctival fornix.

In previous studies, the ocular coil was coated with a SlipSkin ${ }^{\circledR}$ drug eluting coating.[38] Since drug release from the coating was too low for clinical effect, straight pieces of coated wire were inserted in the inner lumen of the ocular coil. Consequently, the ocular coil became more rigid. For short term use (two hours), the ocular coil was felt in the eye, and although patients did not experience discomfort, mild ocular hyperaemia occurred.[39] Due to the increased rigidity and low drug loading, filling alternatives for the inner-lumen of the ocular coil were investigated. Microspheres minimally affected the flexibility of the ocular coil and increased the drug loading capacity of the ocular coil.[40] In parallel, multiple drugs have been tested, i.e. antibiotics (e.g., pradofloxacin [41,42] and chloramphenicol [38]), a mydriatic agent (e.g., atropine [38]), and a colouring dye (e.g., fluorescein [38]). Since the results for short term drug delivery were positive, prolonged drug delivery (e.g., postoperative treatments) was suggested.[42]

\subsubsection{Drug detection method}

Knowledge of the drug delivery capacity and the pharmacokinetic properties of new drug delivery devices in vivo is essential. It shows whether the in vitro translation went well and helps to optimize the device. However, measuring in vivo drug concentrations is challenging. Ocular tissues or fluids cannot be harvested without interfering with the anatomical integrity of the eye (e.g., during intraocular surgery). Due to the limited accessibility of samples from humans, and the destructiveness of the method, pharmacokinetic research is relying on large quantities of animals, e.g. rabbits, dogs, pigs, and monkeys.[43] Therefore, animal experiments have been widely criticized for both, ethical and economic reasons.[44]

Currently, there is no non-invasive drug detection technique available for in vivo qualification and quantification of ocular drugs. The gold standard for drug detection and quantification is HPLC. This separation technique uses the chemical binding characteristics of a drug, to separate the active component. Characterization of the drug is done after separation using spectroscopy (e.g., mass spectroscopy or UVspectroscopy) and quantification of drug is very accurate (ng/mL).[45] Because HPLC only works with fluids, invasive sampling in combination with purification hinders real-time drug detection.

A technique that is potentially suitable for non-invasive, in vivo detection of ocular drugs is Raman spectroscopy. Raman spectroscopy identifies molecules, based on the specific inelastic scattering properties of their rotational and vibrational modes.[46-48] This technique enables real-time detection of molecules without preprocessing and damaging tissue. Compared to infrared (IR) spectroscopy, Raman spectroscopy bears the advantage that molecules do not have to possess a 
permanent dipole moment. Therefore, more molecules can be detected. Furthermore, Raman spectroscopy is not affected by aqueous samples, and absorption bands are better separated (resulting in higher specificity), in contrast to IR and near-infrared spectroscopy, respectively.[49]

\subsubsection{Quantification of ocular redness}

Safety of the ocular coil is essential for a new drug delivery device in order to serve as a valuable alternative to eye drops and to assure high patient compliance. However, objective scoring of the safety is difficult. Safety is related to multiple parameters i.e., tissue damage and ocular redness. To some extent, the degree of redness may reflect the severity of the disease. Redness of the eye is a sign of ocular inflammation.[50] It is regularly observed in contact lens wearers [51], and was also used as a safety outcome parameter in a previous study with the ocular coil.[39] It is an important diagnostic feature for the diagnosis and monitoring of ocular diseases. In this context, quantification of ocular redness can be of use in both clinical and research settings.

Current ocular redness grading methods rely on grading scales, such as the McMonnies and Chapman-Davies scale [52], Efron's grading scale [53], the Institute for Eye Research scale (also known as CCLRU) [54], and the validated bulbar redness scale.[55] However, those methods are highly subjective, do not offer a continuous evaluation scale, and are often not reproducible because the lack of photographic evidence. Hence, there is no gold standard, despite a relatively high number of existing methods. Using an automated tool would increase the objectivity, due to elimination of inter- and intra-observer variability. It would lower the amount of resources (man-power) needed for evaluation of ocular redness, and allows absolute measurements.

\subsection{OCDC project}

In order to develop and explore innovative methods in ocular drug delivery, the Ocular Coil Drug delivery and Comfort (OCDC) project was launched. A proof-ofconcept of the ocular coil was designed to serve as postoperative treatment. As drug, ketorolac tromethamine (a non-steroidal anti-inflammatory drug (NSAID)) was used because of its high effectiveness in the prevention of ocular inflammation $[56,57]$ and its generic (off-patented) availability. Another additional advantage is the availability of a commercial ophthalmic solution (Acular ${ }^{\mathrm{TM}}$, Allergan, Dublin, IR), and the strong Raman activity of ketorolac [58] to compare effectiveness. 


\subsubsection{Proof-of-concept: postoperative treatment of cataract surgery}

Currently, cataract is the most common cause of blindness worldwide.[59] With advancing age, new layers are added to the ocular lens, compressing and hardening the lens. This mechanical alteration of the lens reduces its transparency and the lens becomes opaque or 'cloudy', called cataract.[7,60] In Western society, cataract can easily be treated with a surgical procedure. During this procedure, the cataractous lens is replaced by an intraocular lens (IOL) engineered from polymers. In the Netherlands, about 180,000 cataract surgeries are performed each year.[61] Although modern cataract surgery has a success rate above $92 \%$, cataract surgery still encounters postoperative complications.[62] Technical advances in cataract surgery have reduced the level of trauma to the intraocular tissue; nonetheless, postoperative complications such as infections and intraocular inflammation may occur.[63]

Postoperative inflammation is caused by breakdown of the cell membranes as result of tissue injury. Surgical trauma activates phospholipase A2 that releases arachidonic acid from the cell membrane phospholipids. Arachidonic acid is metabolized by cyclooxygenases (COX1 and COX2) into prostaglandin $\mathrm{G}_{2}\left(\mathrm{PGG}_{2}\right.$ ) that is converted to prostaglandin $\mathrm{H}_{2}\left(\mathrm{PGH}_{2}\right)$. This leads to the formation of more prostaglandins (figure 1-3). The prostaglandins increase vascular permeability and lead to local vasodilation. Symptoms of intraocular inflammation include hyperaemia, miosis, pain, photophobia, reduced visual acuity, and cystoid macular oedema (CME).[64] CME remains one of the most occurring (about 3.4\%) [65] complications after cataract surgery resulting in suboptimal visual acuity.[66,67]

To prevent postoperative complications, patients are treated with eye drops containing antibiotics, corticosteroids and/or NSAIDs. Corticosteroids inhibit phospholipase A2, thereby blocking the release of arachidonic acid. Without arachidonic acid, eicosanoids (such as prostaglandins) will not be produced (figure 1-3).[64] NSAIDs specifically inhibit the activity of the COX enzymes. NSAIDs act on the same inflammatory pathway as corticosteroids, however, NSAIDs only block one-arm of the inflammatory cascade whereas corticosteroids affect both arms (figure 1-3).[64]

A recent European multicentre study (the ESCRS PREMED study) demonstrated that the combination of topical corticosteroids and topical NSAIDs results in the lowest risk of developing CME after cataract surgery. [65] Although cataract surgery is considered one of the most cost-effective health care interventions,[68] noncompliance compromises effectiveness of the prescribed treatment. Post-operative eye drops are administered one to four times daily in a tapering scheme for four weeks.[69] In total, around 70 eye drop administrations take place during the postoperative period. 


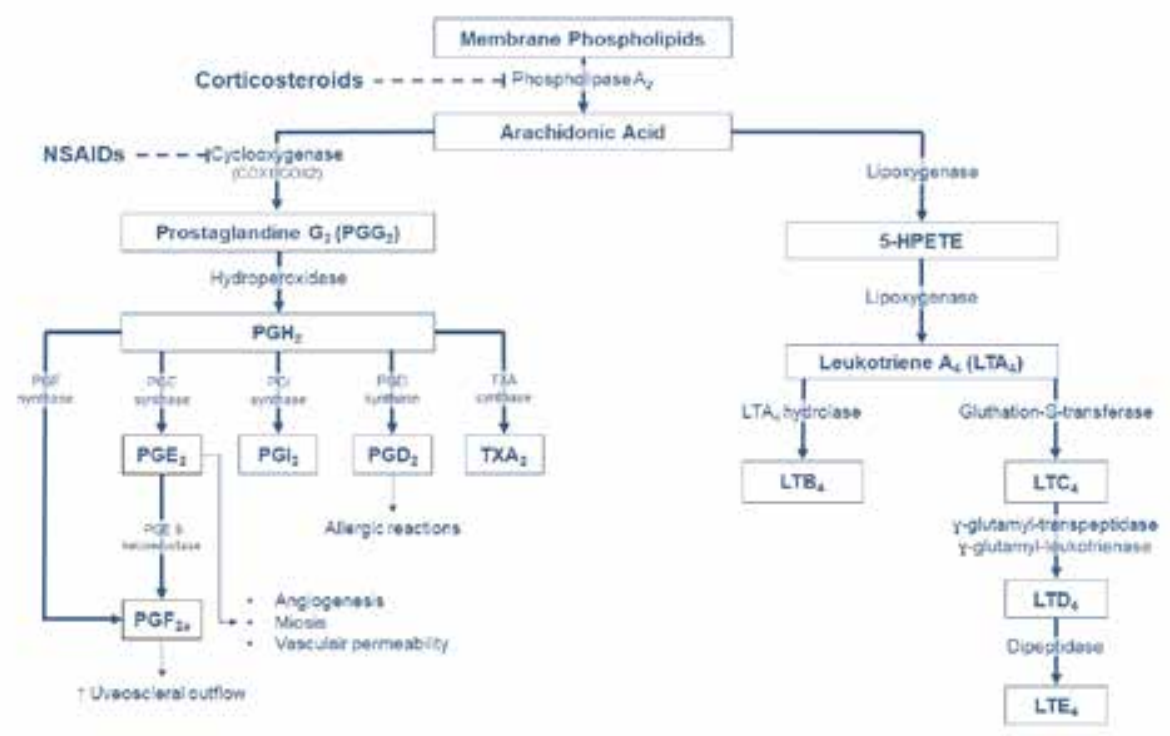

Figure 1-3. Inflammatory pathway and the interaction of corticosteroids and NSAIDs. Corticosteroids block both arms of the pathway and NSAIDs only block the production of COX1 and COX2. Modified from Marcus et al. [70].

\subsubsection{Chemelot InSciTe}

The OCDC project was realized within the framework of the Chemelot Institute for Science and Technology (InSciTe), a public-private partnership of the Maastricht University Medical Center+ (MUMC+), Maastricht University (UM), Eindhoven University of Technology (TU/e), DSM, and the province of Limburg. Chemelot InSciTe was established in 2015 with three main interests in the biomedical field: cardiovascular, orthopaedics, and ophthalmology. Within the ophthalmic field, the OCDC project was first to start.

In the OCDC project, the MUMC+, UM, TU/e, and Eyegle bv. collaborate to deliver a drug delivery device for evaluation in a human comfort and safety study, an animal pharmacokinetic study, and an animal efficacy study.

\subsection{Aims and outline of the thesis}

The aim of this thesis is to provide a clear understanding of the mechanical and functional properties of the ocular coil. Furthermore, innovative techniques in ocular drug detection and objective scoring of ocular redness have been investigated in relation to the ocular coil.

In chapter 2, we reviewed the current state-of-the-art regarding topical ocular drug delivery devices. Different categories of drug delivery devices including resorbable 
devices, oval- and ring-shaped devices, rod-shaped devices, punctum plugs, contact lenses, and corneal shields were described. Most of these non-invasive devices have been developed in order to remove the daily burden of instilling eye drops and, therefore, to improve patient compliance.

To improve patient compliance and drug delivery, the ocular coil was developed.[3842] The ocular coil is a coiled and coated wire that is filled with drug-loaded microspheres. The technical details of the ocular coil are described in chapter 3. First, we designed multiple prototypes of the ocular coil with ranging wire thicknesses and outer diameters. Those prototypes were tested on flexibility and their gap-sizes (i.e. the size between the ocular coil windings) were calculated. With these calculated gap-sizes, potential escape of microspheres between the ocular coil windings was prevented. This was experimentally tested by fixing the ocular coil on a bending and stretching platform. Furthermore, we developed an ocular coil filled with ketorolac encapsulated microspheres and tested the in vitro drug release in an artificial lacrimal system. In chapter 4, we evaluated the pharmacokinetics of the drug loaded ocular coil in New Zealand White rabbits. This was followed by evaluation of the efficacy of the ketorolac loaded ocular coil. After inducing a (surgical) ocular trauma, the rabbit's eye was treated with either a drug loaded ocular coil, eye drops, or was left untreated.

The findings of our first-in-man study with the placebo ocular coil are presented in chapter 5. We tested two designs of ocular coils (a straight and a curved version) in two cohorts of healthy volunteers. The volunteers were asked to wear the ocular coil in one eye for 28 days. During several follow-up visits, safety and comfort of the ocular coil was evaluated through slit lamp examinations, questionnaires, and imaging.

In parallel, we developed two new detection methods to evaluate the ocular coil. Chapter 6, 6a, and $\mathbf{6 b}$ describe a new in vivo method for the detection of ketorolac tromethamine using Raman spectroscopy. First, we created a Raman spectroscope set-up for in vitro samples. Detection of ketorolac using in vitro Raman spectroscopy was compared to high-performance liquid chromatography (HPLC). Second, the in vivo detection of ketorolac using Raman spectroscopy was evaluated in New Zealand White rabbits.

Chapter 7 discusses quantification of ocular redness using deep-learning technology. Ocular redness was, amongst others, one of the parameters that was used to evaluate the safety of the ocular coil. In chapter $\mathbf{8}$, a general discussion is provided and the impact of the research is outlined in chapter $\mathbf{9}$. 


\subsection{References}

1. American Academy of Ophthalmology., Fundamentals and principles of ophthalmology, in Basic and clinical science course. 2014-2015, American Academy of Ophthalmology: San Francisco, CA. p. 449.

2. Moore, K.L., Dalley, A.F., and Agur, A.M.R., Clinically oriented anatomy. 6th ed. 2010, Philadelphia: Wolters Kluwer/Lippincott Williams \& Wilkins. xxix, p.1134.

3. Levin, L.A. and Kaufman, P.L., Adler's physiology of the eye : clinical application. 11th ed. 2011, Edinburgh ; New York: Saunders/Elsevier. xii, p. 795.

4. Goel, M., Picciani, R.G., Lee, R.K., and Bhattacharya, S.K., Aqueous humor dynamics: a review. Open Ophthalmol J, 2010. 4: p. 52-59.

5. American Academy of Ophthalmology., Retina and Vitreous, in Basic and clinical science course. 2014-2015, American Academy of Ophthalmology: San Francisco, CA. p. 438.

6. American Academy of Ophthalmology., Refractive Surgery, in Basic and clinical science course. 2014-2015, American Academy of Ophthalmology: San Francisco, CA. p. 272.

7. American Academy of Ophthalmology., Lens and Cataract, in Basic and clinical science course. 2014-2015, American Academy of Ophthalmology: San Francisco, CA. p. 271.

8. American Academy of Ophthalmology., Clinical Optics, in Basic and clinical science course. 2014-2015, American Academy of Ophthalmology: San Francisco, CA. p. 383.

9. Prausnitz, M.R. and Noonan, J.S., Permeability of cornea, sclera, and conjunctiva: a literature analysis for drug delivery to the eye. J Pharm Sci, 1998. 87(12): p. 1479-1488.

10. Ambati, J., Canakis, C.S., Miller, J.W., Gragoudas, E.S., Edwards, A., Weissgold, D.J., Kim, I., Delori, F.C., and Adamis, A.P., Diffusion of high molecular weight compounds through sclera. Invest Ophthalmol Vis Sci, 2000. 41(5): p. 1181-1185.

11. Hughes, P.M., Olejnik, O., Chang-Lin, J.E., and Wilson, C.G., Topical and systemic drug delivery to the posterior segments. Adv Drug Deliv Rev, 2005. 57(14): p. 2010-2032.

12. Bauer, N.J., Motamedi, M., Wicksted, J.P., March, W.F., Webers, C.A., and Hendrikse, F., Noninvasive assessment of ocular pharmacokinetics using Confocal Raman Spectroscopy. J Ocul Pharmacol Ther, 1999. 15(2): p. 123-134.

13. Lee, S.J., Kim, S.J., Kim, E.S., Geroski, D.H., McCarey, B.E., and Edelhauser, H.F., Transscleral permeability of Oregon green 488. J Ocul Pharmacol Ther, 2008. 24(6): p. 579-586.

14. Del Amo, E.M. and Urtti, A., Rabbit as an animal model for intravitreal pharmacokinetics: Clinical predictability and quality of the published data. Exp Eye Res, 2015. 137: p. 111-124.

15. Shikamura, Y., Yamazaki, Y., Matsunaga, T., Sato, T., Ohtori, A., and Tojo, K., Hydrogel Ring for Topical Drug Delivery to the Ocular Posterior Segment. Curr Eye Res, 2016. 41(5): p. 653661.

16. Zhang, W., Prausnitz, M.R., and Edwards, A., Model of transient drug diffusion across cornea. J Control Release, 2004. 99(2): p. 241-258.

17. Ahmed, I. and Patton, T.F., Importance of the noncorneal absorption route in topical ophthalmic drug delivery. Invest Ophthalmol Vis Sci, 1985. 26(4): p. 584-587.

18. Kim, H., Robinson, M.R., Lizak, M.J., Tansey, G., Lutz, R.J., Yuan, P., Wang, N.S., and Csaky, K.G., Controlled drug release from an ocular implant: an evaluation using dynamic threedimensional magnetic resonance imaging. Invest Ophthalmol Vis Sci, 2004. 45(8): p. 27222731.

19. Del Amo, E.M., Rimpela, A.K., Heikkinen, E., Kari, O.K., Ramsay, E., Lajunen, T., Schmitt, M., Pelkonen, L., Bhattacharya, M., Richardson, D., Subrizi, A., Turunen, T., Reinisalo, M., Itkonen, J., Toropainen, E., Casteleijn, M., Kidron, H., Antopolsky, M., Vellonen, K.S., Ruponen, M., and Urtti, A., Pharmacokinetic aspects of retinal drug delivery. Prog Retin Eye Res, 2017. 57: p. 134-185.

20. Kidron, H., Del Amo, E.M., Vellonen, K.S., and Urtti, A., Prediction of the vitreal half-life of small molecular drug-like compounds. Pharm Res, 2012. 29(12): p. 3302-3311.

21. Urtti, A., Challenges and obstacles of ocular pharmacokinetics and drug delivery. Adv Drug Deliv Rev, 2006. 58(11): p. 1131-1135.

22. Farkouh, A., Frigo, P., and Czejka, M., Systemic side effects of eye drops: a pharmacokinetic perspective. Clin Ophthalmol, 2016. 10: p. 2433-2441.

23. Kaszli, F.A. and Krieglstein, G.K., Tear film deficiencies pharmacology of eye drops and toxicity. Curr Opin Ophthalmol, 1996. 7(4): p. 12-16.

24. Palmer, R.M. and Kaufman, H.E., Tear film, pharmacology of eye drops, and toxicity. Curr Opin Ophthalmol, 1995. 6(4): p. 11-16. 
25. Ramsay, E., Del Amo, E.M., Toropainen, E., Tengvall-Unadike, U., Ranta, V.P., Urtti, A., and Ruponen, M., Corneal and conjunctival drug permeability: Systematic comparison and pharmacokinetic impact in the eye. Eur J Pharm Sci, 2018. 119: p. 83-89.

26. Pelkonen, L., Tengvall-Unadike, U., Ruponen, M., Kidron, H., Del Amo, E.M., Reinisalo, M., and Urtti, A., Melanin binding study of clinical drugs with cassette dosing and rapid equilibrium dialysis inserts. Eur J Pharm Sci, 2017. 109: p. 162-168.

27. Olthoff, C.M., Hoevenaars, J.G., van den Borne, B.W., Webers, C.A., and Schouten, J.S., Prevalence and determinants of non-adherence to topical hypotensive treatment in Dutch glaucoma patients. Graefes Arch Clin Exp Ophthalmol, 2009. 247(2): p. 235-243.

28. An, J.A., Kasner, O., Samek, D.A., and Levesque, V., Evaluation of eyedrop administration by inexperienced patients after cataract surgery. J Cataract Refract Surg, 2014. 40(11): p. 18571861.

29. Bertens, C.J.F., Gijs, M., van den Biggelaar, F., and Nuijts, R., Topical drug delivery devices: A review. Exp Eye Res, 2018. 168: p. 149-160.

30. Gaudana, R., Ananthula, H.K., Parenky, A., and Mitra, A.K., Ocular drug delivery. AAPS J, 2010. 12(3): p. 348-360.

31. Lee, S.B., Geroski, D.H., Prausnitz, M.R., and Edelhauser, H.F., Drug delivery through the sclera: effects of thickness, hydration, and sustained release systems. Exp Eye Res, 2004. 78(3): p. 599-607.

32. Urtti, A. and Salminen, L., Minimizing systemic absorption of topically administered ophthalmic drugs. Surv Ophthalmol, 1993. 37(6): p. 435-456.

33. Hermann, M.M., Ustundag, C., and Diestelhorst, M., Electronic compliance monitoring of topical treatment after ophthalmic surgery. Int Ophthalmol, 2010. 30(4): p. 385-390.

34. Salyani, A. and Birt, C., Evaluation of an eye drop guide to aid self-administration by patients experienced with topical use of glaucoma medication. Can J Ophthalmol, 2005. 40(2): p. 170174.

35. Nordmann, J.P., Baudouin, C., Renard, J.P., Denis, P., Lafuma, A., Laurendeau, C., Jeanbat, V., and Berdeaux, G., Measurement of treatment compliance using a medical device for glaucoma patients associated with intraocular pressure control: a survey. Clin Ophthalmol, 2010. 4: p. 731-739.

36. Rajurkar, K., Dubey, S., Gupta, P.P., John, D., and Chauhan, L., Compliance to topical antiglaucoma medications among patients at a tertiary hospital in North India. J Curr Ophthalmol, 2018. 30(2): p. 125-129.

37. Davis, S.A., Sleath, B., Carpenter, D.M., Blalock, S.J., Muir, K.W., and Budenz, D.L., Drop instillation and glaucoma. Curr Opin Ophthalmol, 2018. 29(2): p. 171-177.

38. Pijls, R.T., Hanssen, H.H., Nuijts, R.M., and Koole, L.H., Flexible coils with a drug-releasing hydrophilic coating: a new platform for controlled delivery of drugs to the eye? Biomed Mater Eng, 2004. 14(4): p. 383-393.

39. Pijls, R.T., Cruysberg, L.P., Nuijts, R.M., Dias, A.A., and Koole, L.H., Capacity and tolerance of a new device for ocular drug delivery. Int J Pharm, 2007. 341(1-2): p. 152-161.

40. Pijls, R.T., Hanssen, H.H., Nuijts, R.M., Daube, G.W., and Koole, L.H., In vivo tolerance and kinetics of a novel ocular drug delivery device. J Control Release, 2006. 116(2): p. e47-49.

41. Pijls, R.T., Lindemann, S., Nuijts, R.M.M.A., Daube, G.W., and Koole, L.H., Pradofloxacin release from the OphthaCoil: a new device for sustained delivery of drugs to the eye. Journal of Drug Delivery Science and Technology, 2007. 17(1): p. 87-91.

42. Pijls, R.T., Sonderkamp, T., Daube, G.W., Krebber, R., Hanssen, H.H., Nuijts, R.M., and Koole, L.H., Studies on a new device for drug delivery to the eye. Eur J Pharm Biopharm, 2005. 59(2): p. 283-288.

43. Schoenwald, R.D., Ocular drug delivery. Pharmacokinetic considerations. Clin Pharmacokinet, 1990. 18(4): p. 255-269.

44. Hornof, M., Toropainen, E., and Urtti, A., Cell culture models of the ocular barriers. Eur J Pharm Biopharm, 2005. 60(2): p. 207-225.

45. Buckley, K. and Ryder, A.G., Applications of Raman Spectroscopy in Biopharmaceutical Manufacturing: A Short Review. Appl Spectrosc, 2017. 71(6): p. 1085-1116.

46. Raman, C.V. and Krishnan, K.S., A New Type of Secondary Radiation. Nature, 1928. 121: p. 501-502.

47. Bauer, N.J.C., Potential applications of confocal Raman spectroscopy in ophthalmology, in Ophthalmology. 1999, Datawyse: Maastricht. p. 160.

48. Erckens, R.J., Development of the Raman spectroscopic technique for in vivo applications in the eye, in Ophthalmology. 2001, Unigraphic: Maastricht. p. 140. 
49. Gowen, A.A., Marini, F., Tsuchisaka, Y., De Luca, S., Bevilacqua, M., O'Donnell, C., Downey, G., and Tsenkova, R., On the feasibility of near infrared spectroscopy to detect contaminants in water using single salt solutions as model systems. Talanta, 2015. 131: p. 609-618.

50. Cronau, H., Kankanala, R.R., and Mauger, T., Diagnosis and management of red eye in primary care. Am Fam Physician, 2010. 81(2): p. 137-144.

51. McMonnies, C.W. and Chapman-Davies, A., Assessment of conjunctival hyperemia in contact lens wearers. Am J Optom Physiol Op, 1987. 64(4): p. 246-250.

52. McMonnies, C.W. and Chapman-Davies, A., Assessment of conjunctival hyperemia in contact lens wearers. Part II. Am J Optom Physiol Opt, 1987. 64(4): p. 251-255.

53. Efron, N., Grading scales for contact lens complications. Ophthalmic Physiol Opt, 1998. 18(2): p. $182-186$.

54. IER. IER grading scales. 2007 [cited 2018 Jan 18]; Available from: http://www.ier.org.au/.

55. Schulze, M.M., Jones, D.A., and Simpson, T.L., The development of validated bulbar redness grading scales. Optom Vis Sci, 2007. 84(10): p. 976-983.

56. Waterbury, L.D. and Flach, A.J., Comparison of ketorolac tromethamine, diclofenac sodium, and loteprednol etabonate in an animal model of ocular inflammation. J Ocul Pharmacol Ther, 2006. 22(3): p. 155-159.

57. Bucci, F.A., Jr., Waterbury, L.D., and Amico, L.M., Prostaglandin E2 inhibition and aqueous concentration of ketorolac $0.4 \%$ (acular LS) and nepafenac $0.1 \%$ (nevanac) in patients undergoing phacoemulsification. Am J Ophthalmol, 2007. 144(1): p. 146-147.

58. Elshout, M., Erckens, R.J., Webers, C.A., Beckers, H.J., Berendschot, T.T., de Brabander, J., Hendrikse, F., and Schouten, J.S., Detection of Raman spectra in ocular drugs for potential in vivo application of Raman spectroscopy. J Ocul Pharmacol Ther, 2011. 27(5): p. 445-451.

59. Bourne, R.R., Stevens, G.A., White, R.A., Smith, J.L., Flaxman, S.R., Price, H., Jonas, J.B., Keeffe, J., Leasher, J., Naidoo, K., Pesudovs, K., Resnikoff, S., Taylor, H.R., and Vision Loss Expert, G., Causes of vision loss worldwide, 1990-2010: a systematic analysis. Lancet Glob Health, 2013. 1(6): p. e339-349.

60. Harding, J.J., Viewing molecular mechanisms of ageing through a lens. Ageing Research Reviews, 2002. 1(3): p. 465-479.

61. Henry, Y.P. Practice styles and Preferences of Dutch Cataract and Refractive surgeons. 2016 survey. in NOG 2018. 2018. Groningen.

62. (UK), N.I.f.H.a.C.E., Cataracts in adults: management. 2017, National Institute for Health and Care Excellence (UK): London. p. 13, Postoperative assessment.

63. Simone, J.N. and Whitacre, M.M., Effects of anti-inflammatory drugs following cataract extraction. Curr Opin Ophthalmol, 2001. 12(1): p. 63-67.

64. Kohnen, T., Treating inflammation after lens surgery. J Cataract Refract Surg, 2015. 41(10): $p$. 2035.

65. Wielders, L.H.P., Schouten, J., Winkens, B., van den Biggelaar, F., Veldhuizen, C.A., Findl, O., Murta, J.C.N., Goslings, W.R.O., Tassignon, M.J., Joosse, M.V., Henry, Y.P., Rulo, A.H.F., Guell, J.L., Amon, M., Kohnen, T., Nuijts, R., and Group, E.P.S., European multicenter trial of the prevention of cystoid macular edema after cataract surgery in nondiabetics: ESCRS PREMED study report 1. J Cataract Refract Surg, 2018. 44(4): p. 429-439.

66. McCafferty, S., Harris, A., Kew, C., Kassm, T., Lane, L., Levine, J., and Raven, M., Pseudophakic cystoid macular edema prevention and risk factors; prospective study with adjunctive once daily topical nepafenac $0.3 \%$ versus placebo. BMC Ophthalmol, 2017. 17(1): p. 16.

67. Kim, S.J., Schoenberger, S.D., Thorne, J.E., Ehlers, J.P., Yeh, S., and Bakri, S.J., Topical Nonsteroidal Anti-inflammatory Drugs and Cataract Surgery: A Report by the American Academy of Ophthalmology. Ophthalmology, 2015. 122(11): p. 2159-2168.

68. Wang, W., Yan, W., Fotis, K., Prasad, N.M., Lansingh, V.C., Taylor, H.R., Finger, R.P., Facciolo, D., and He, M., Cataract Surgical Rate and Socioeconomics: A Global Study. Invest Ophthalmol Vis Sci, 2016. 57(14): p. 5872-5881.

69. O'Brien, T.P., Emerging guidelines for use of NSAID therapy to optimize cataract surgery patient care. Curr Med Res Opin, 2005. 21(7): p. 1131-1137.

70. Marcus, A.J., The eicosanoids in biology and medicine. J Lipid Res, 1984. 25(13): p. 15111516. 
General introduction 


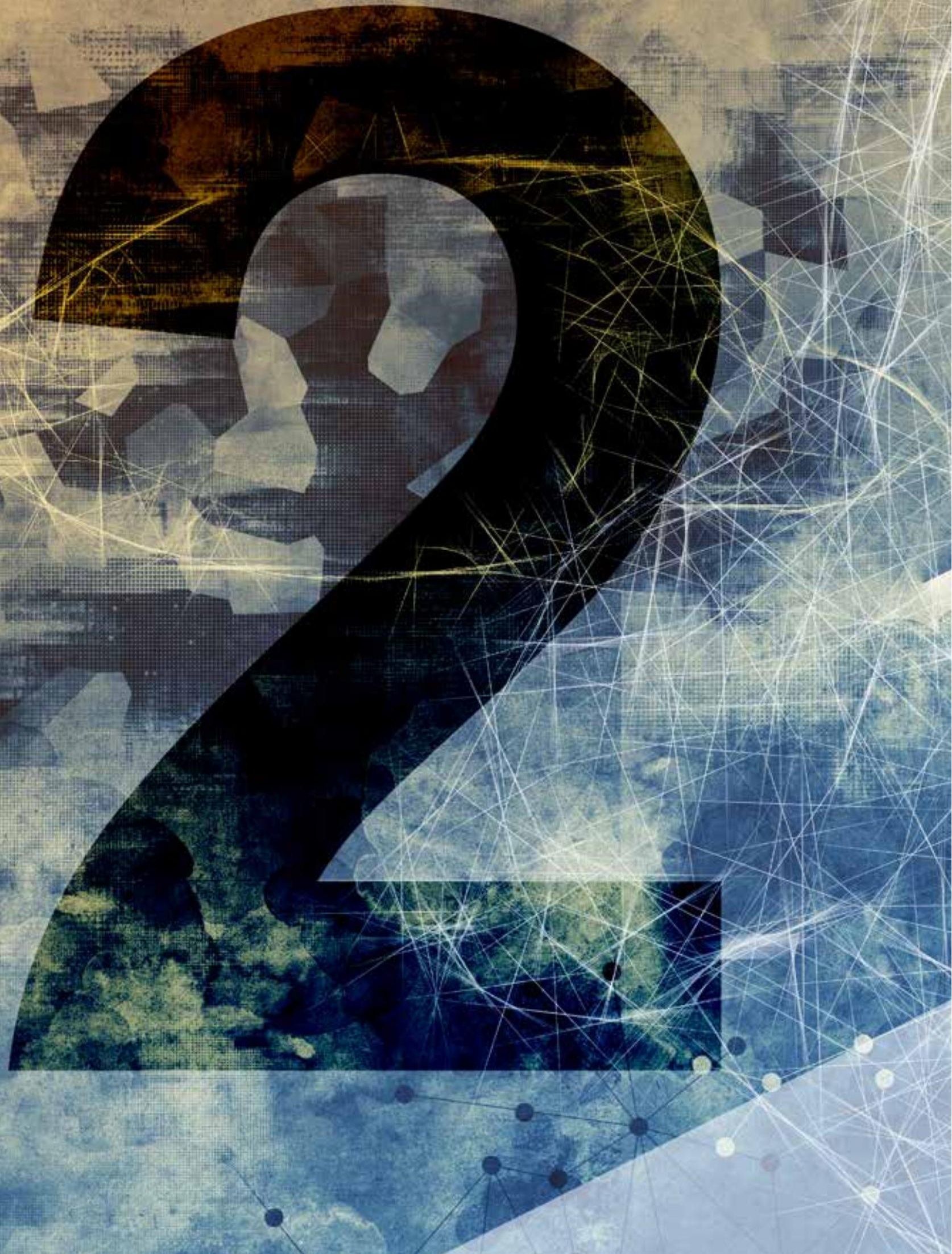




\section{Abstract}

For the treatment and prevention of ocular diseases, most patients are treated with conventional drug delivery formulations such as eye drops or ointments. However, eye drops and ointments suffer from low patient compliance and low effective drug concentration at the target site. Therefore, new medical devices are being explored to improve drug delivery to the eye. Over the years, various delivery devices have been developed including resorbable devices, oval- and ring- shaped devices, rodshaped devices, punctum plugs, and contact lenses and corneal shields. Only a few devices (e.g. Mydriasert ${ }^{\circledR}$, Ozurdex $^{\circledast}$, Surodex $^{\circledR}$, lluvien ${ }^{\circledast}$, Lacrisert $^{\circledR}$ and Retisert ${ }^{\circledast}$ ) have made it to the market while others are being investigated in clinical trials.

Altogether, there is a need for enhanced topical drug delivery. Only by working together (academia, industry, and authorities) and by exploring parallel strategies (new drug delivery devices, enhanced drug formulations, better understanding of the pharmacokinetic properties), the therapeutic effect of drug treatments can be improved. 


\subsection{Introduction}

Due to the increased prevalence of ocular diseases in the aging population (such as presbyopia, cataract, dry eyes, and glaucoma), there is an increased demand for treatment of eye diseases. Aging of the lens may lead to loss of accommodation (presbyopia) or protein aggregation (cataract). Although revolutionary treatments have been discovered, for example lipoic acid (LA) to prevent the loss of accommodation[1], compound 29 and lanosterol to restore lens transparency in (congenital) cataract[2,3] or Kinostat ${ }^{\circledR}$ to prevent cataract in diabetic dogs[4], they mostly require life-long or long-term administration of eye drops. Also, ocular therapies for dry eyes consist of life-long topical administered artificial tears, gels, ointments or lubricants to relieve symptoms. [5] Glaucoma therapy consists of topical applied beta blockers or prostaglandin analogs, laser therapy or surgery to lower the intra ocular pressure (IOP).[6-8]

Ocular surgeries range from routine cataract extraction and lens implantation, the most commonly performed surgery worldwide, to rarely performed surgeries such as keratoprosthesis. Due to an increase in the number of surgeries, the odds of infections and inflammations increase if postoperative care is not taken care of properly. A variety of postoperative complications may occur such as cystoid macular edema (CME) (incidence 1.2\% to 3.5\%) after cataract surgery.[9-11] In order to prevent postoperative complications, patients are treated with ophthalmic anti-inflammatory drugs such as corticosteroids or non-steroidal anti-inflammatory drugs (NSAIDs), and antibiotics.[10-16]

In all ocular therapies and surgeries involving medicines, the route of drug delivery plays an important role. Although systemic drugs do reach the ocular tissues[17], high doses are required which often lead to side effects. Therefore, the most preferred way of drug delivery to the eye is topically. Drugs administered topically are absorbed through the corneal or non-corneal absorption route (figure 2-1). Drug molecules with high corneal permeability (e.g. small molecules with a hydroxyl group) prefer the corneal route (figure 2-1).[18,19] This route starts with passive diffusion of drug molecules via the epithelium, through the stroma and endothelium into the anterior chamber, where the drug will exert its pharmacological function [1922] or bind to the melanin pigment in the iris and ciliary body[23] or to plasma proteins[24] to prolong its pharmacological function. Remaining drugs and drug waste products will be cleared via the trabecular meshwork through Schlemm's channel into the systemic blood circulation (conventional pathway) or via the iris to the uveoscleral tissue (unconventional pathway) and subsequently into the systemic blood circulation. [25-28] A minor part of the drug (dependent on the molecular weight and lipophilicity) will reach the posterior chamber via penetration of the iris and diffusion via the aqueous humor flow resulting in drug concentrations in the vitreous 
which are 10 and 100 times less than in the aqueous humor and cornea, respectively.[18] It must be noted that these pharmacokinetics can be altered due to eye rotations[29,30] and ocular diseases.[22,31,32]

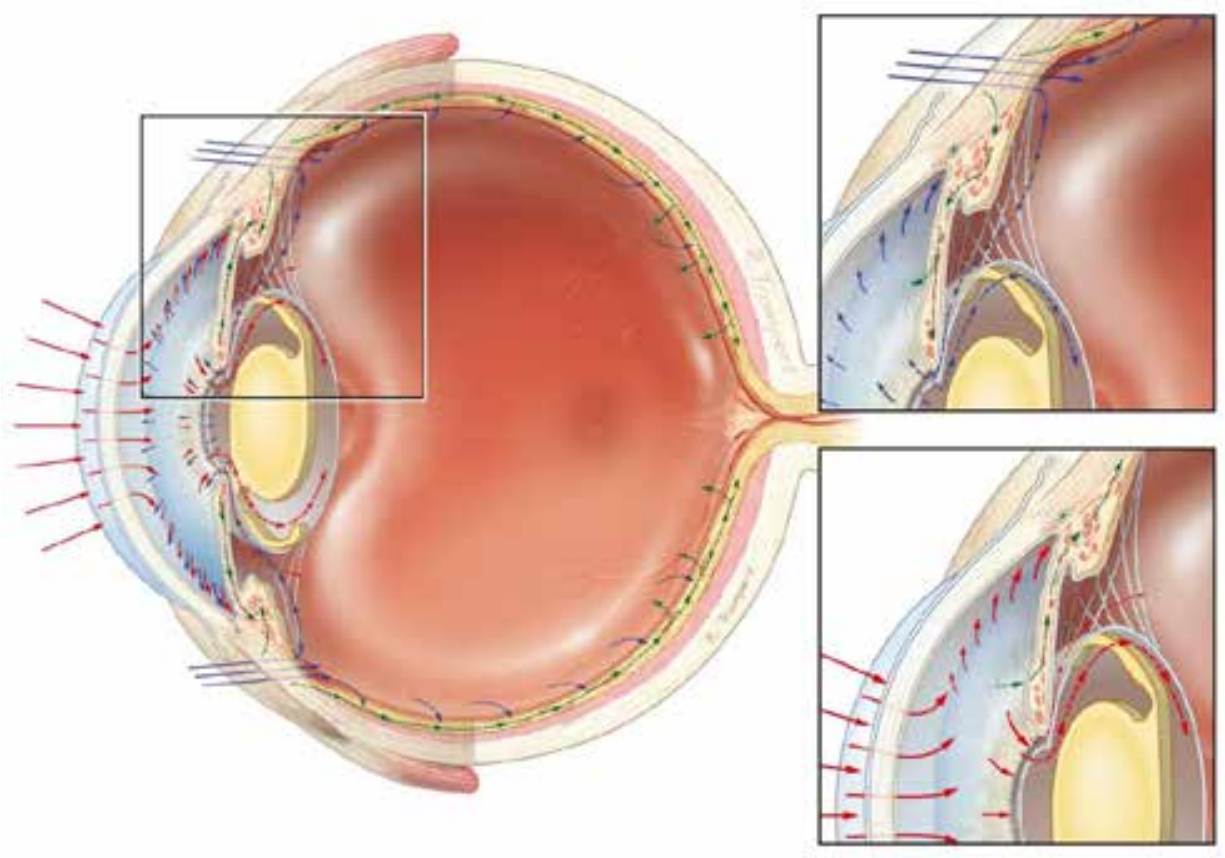

Figure 2-1. Drug delivery routes in the eye. Red arrows show the corneal delivery route with penetration through the cornea into the anterior chamber and clearance via the trabecular meshwork (anterior elimination route). A small part of the drug (dependent on the molecular weight and lipophilicity) will migrate into tissue of the iris or bind to the melanin pigment, from where it can get into the posterior chamber (green arrows). The lower inset shows a magnification of the corneal route. Blue arrows show the non-corneal delivery route, drugs penetrate via the conjunctiva through the sclera into the choroid and to some extend through the retina into the vitreous cavity (green arrows). In the vitreous cavity, small drug molecules $(<2 \mathrm{~nm}$ ) will be cleared via the blood-retina barrier (posterior elimination route, blue arrows). Most of the drug will reach the anterior chamber via aqueous humor flow. The upper inset provides a magnification of the non-corneal drug delivery route and drug transport or diffusion into the anterior chamber. The figure is drawn with an IOL to address that an IOL due to its anatomical position could be used as drug delivery system.

Drugs with low corneal permeability (e.g. large molecules and proteins) will penetrate the eye via the conjunctiva and/or the sclera, the so-called non-corneal absorption route (figure 2-2).[25,33-36] This route delivers drugs to the vitreous cavity via passive diffusion. Moreover, it is hypothesized that active transport also plays a role. Drugs penetrate or diffuse via the conjunctiva through the sclera into the choroid and through the retina (retinal pigment epithelium (RPE) cells and retinal capillary 
endothelial cells) into the vitreous cavity.[20,21,37,38] Once inside the vitreous chamber the drug will be transported towards the anterior chamber by the flow of aqueous humor or will be cleared via passive diffusion (determined by the LogD7.4 and hydrogen bonding capacity of the drug) by the RPE and retinal capillary endothelial cells (which form the blood-retinal barrier) through the choroidal circulation into the systemic blood flow.[18,25,39] The non-corneal absorption route delivers 20 times lower drug concentration into the anterior chamber compared to the corneal absorption route.[37]

Conventional ocular dosage forms, such as eye drop solutions (figure 2-2,10) and ointments, account for approximately $90 \%$ of currently marketed ophthalmic pharmaceuticals. Their biggest advantages are ease of administration and low costs. Moreover, eye drops are well accepted by most patients and have a rapid and localized drug action.[40] Nevertheless, eye drop delivery is associated with several disadvantages. Next to systemic side effects[41] and toxicity[42], the main disadvantages of eye drop delivery are low drug bioavailability and poor patient compliance (Table 2-1). Pre-corneal loss of the drug (by systemic conjunctival elimination, blinking, induced lacrimation, the tear film and rapid tear turnover, see Table 2-1) results in a very low ocular bioavailability of the drug at its target destination. Typically, less than $5 \%$ of the total administered dose reaches the anterior chamber.[34,43,44] In addition, high aqueous humor turnover washes out the drugs relatively fast $(1.0 \%-1.5 \%$ of the anterior chamber volume per minute)[26,40], and drug-melanin binding could affect the pharmacological function of the drug[18,24] (Table 2-1). Another excreting factor influencing pre-corneal loss is lacrimal clearance, which clears aqueous solutions in 60 seconds and higher viscose solutions such as hydroxypropyl methylcellulose in 4 minutes (depending on its concentration).[45]

In order to maintain minimum inhibitory concentrations, ocular drugs need to be administered frequently resulting in poor patient compliance[46-48](Table 2-1). Low patient compliance is mainly caused by incorrect instillment (time wise and dropwise) of the eye drops, which typically occurs in more cases than assumed by physicians and patients[48-51](Table 2-1). Low patient compliance may result in an increased incidence and severity of postoperative complications (such as inflammation) and under-treatment of ocular diseases (such as glaucoma), which create a significant burden for the health care system. Therefore, new methods for ocular drug delivery are needed within the ophthalmic field. In this review, an overview of current state of knowledge on topical drug delivery devices is provided. 


\section{Chapter 2}

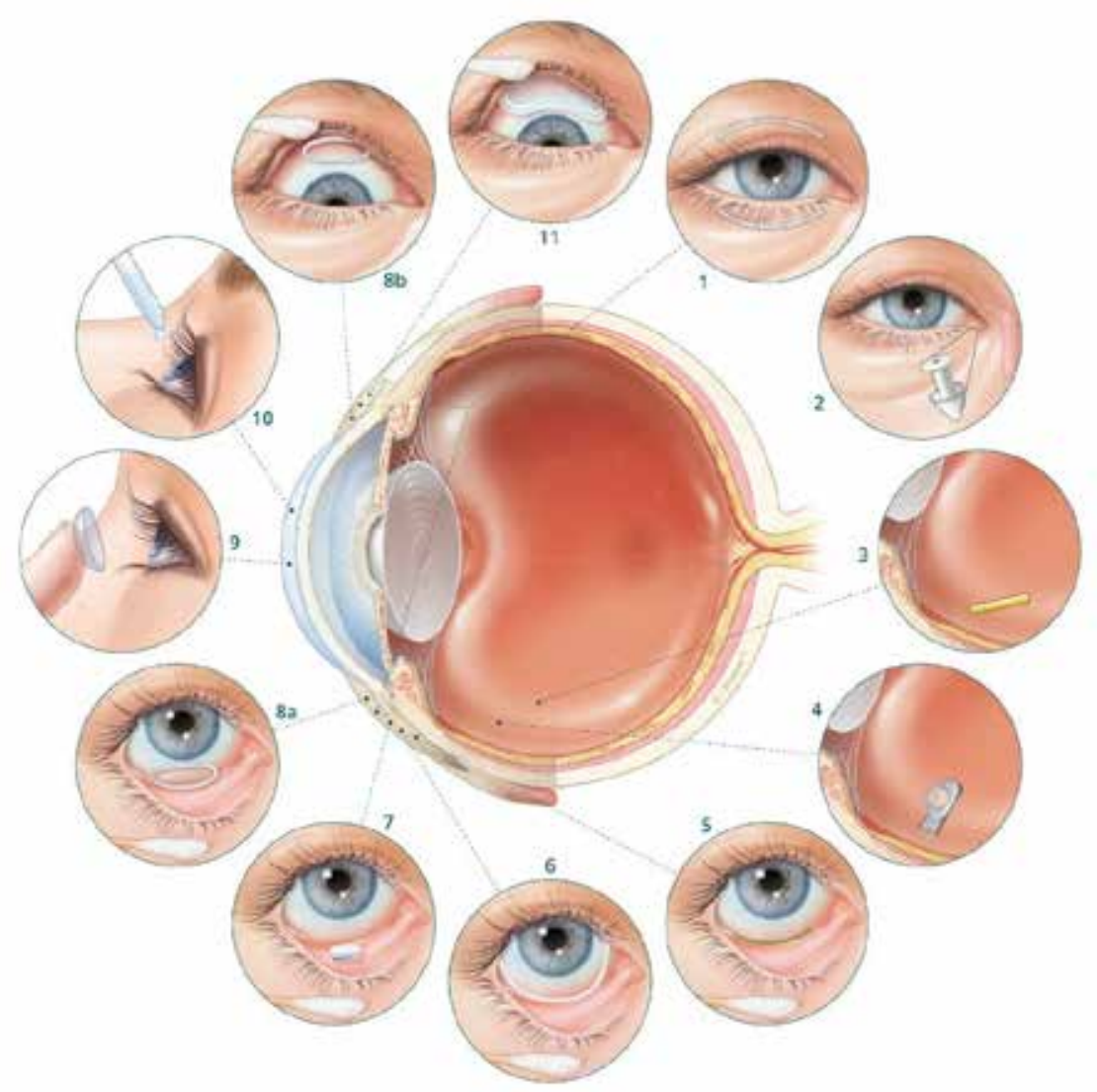

Figure 2-2. Overview of drug delivery devices.

1. Conjunctival insert Ocufit $S R^{\circledR}$, which can be placed in both, the inferior and superior conjunctival fornix.

2. Punctum plug such as Dextenza ${ }^{\circledR}$ or Evolute ${ }^{\circledR}$ should be inserted in the inferior punctum.

3. Intravitreal insert such as Illuven ${ }^{\circledR}$ or Surodex ${ }^{\circledR}$ which are implanted or injected into the vitreous chamber.

4. Intravitreal insert Retrisert ${ }^{\circledR}$, which is implanted into the vitreous chamber and is stitched to the sclera.

5. Conjunctival insert OphthaCoil, is a device placed behind the lower eyelid in the fornix of the conjunctiva.

6. Conjunctival insert Helios ${ }^{\mathrm{TM}}$. The ring is placed round the globe into the inferior and superior conjunctival fornix.

7. Conjunctival tablet, Lacrisert ${ }^{\circledR}$ or Mydriasert $^{\circledR}$. Both tablets are placed in the inferior conjunctival fornix.

8. Conjunctival insert Ocusert ${ }^{\circledR}$, can be placed in the inferior (8a) and superior (8b) conjunctival fornix.

9. Contact lens for drug delivery, to be placed on the cornea.

10. Eye drops.

11. Conjunctival insert for the superior conjunctiva, TODDD ${ }^{\mathrm{TM}}$. 
Table 2-1. Examples of disadvantages related to eye drops.

\begin{tabular}{|c|c|}
\hline Disadvantage & Reference \\
\hline Systemic side effects & {$[41]$} \\
\hline Toxicity & [42] \\
\hline $\begin{array}{l}\text { Low ocular bioavailability: } \\
\text { Pre-corneal loss of the drug due to systemic } \\
\text { conjunctival elimination, blinking, induced } \\
\text { lacrimation, the tear film and rapid tear turnover. }\end{array}$ & {$[34,35,43,44,52]$} \\
\hline $\begin{array}{l}\text { Low ocular bioavailability: } \\
\text { Fast drug wash-out due to high aqueous humor } \\
\text { turnover or lacrimal clearance }\end{array}$ & {$[26,40,45]$} \\
\hline $\begin{array}{l}\text { Low ocular bioavailability: } \\
\text { Drug binding to proteins }\end{array}$ & {$[18,24]$} \\
\hline $\begin{array}{l}\text { Poor patient compliance: } \\
\text { Drop instillment is (too) frequent }\end{array}$ & [48-51] \\
\hline $\begin{array}{l}\text { Poor patient compliance: } \\
\text { Drop instillment is incorrectly performed }\end{array}$ & [48-51] \\
\hline
\end{tabular}

\subsection{Topical Drug delivery devices}

In response to the obstacles of conventional drug dosage forms, alternatives have been explored. These are mainly drug-loaded medical devices which use the noncorneal absorption route (figure 2-2). Historically, the first precursors of ocular inserts were small sections of filter paper impregnated with drug solutions (e.g., atropine sulfate, pilocarpine hydrochloride).[53] In the late 1800s, polymeric inserts containing cocaine for local anesthesia were already used in the United Kingdom (U.K.).[54] In the 1970s, soluble ophthalmic drug inserts (SODIs) were introduced in the Union of Soviet Socialist Republics (U.S.S.R.).[55] SODIs were oval plates made from polyvinyl alcohol (PVA) and impregnated with several drugs. According to a trial in which 500 patients participated, SODIs had good tolerance.[55,56] So far, most drug delivery devices have been explored for posterior drug delivery (e.g. intraocular pressure lowering drugs for glaucoma) and research has been primarily initiated by the industry. Only vitreous implants have made it to the market, as episcleral implants were not able to deliver enough drugs into the vitreous cavity.[32,38] A few vitreous implants are commercially available, including Ozurdex ${ }^{\circledR}$ (Allergan inc., Irvine, California, USA) (figure 2-2,3) (0.7 mg dexamethasone for 60-90 days), Surodex ${ }^{\circledR}$ (Oculex Pharmaceuticals Inc. taken over by Allergan Inc., Irvine, California, USA in 2003) (60 $\mu \mathrm{g}$ dexamethasone for 7-10 days), lluvien ${ }^{\circledR}$ (Alimera Sciences Inc., Alpharetta, Georgia, USA) (figure 2-2,3) (0.23-0.45 $\mu \mathrm{g} / \mathrm{day}$ fluocinolone for 18-36 months) and Retisert ${ }^{\circledR}$ (Bausch \& Lomb, Bridgewater, New 
Jersey, USA) (figure 2-2,4) (0.59 mg fluocinolone acetonide for 30 months). These devices are placed in the vitreous chamber by implantation or injection.

Today, there is also interest in exploring these devices for the anterior segment. However, it is difficult to create an implant for the anterior chamber, since it will move due to the low viscosity of the aqueous humor, and thereby causing irreversible damage to the endothelial cells.[57] Although, when Surodex ${ }^{\circledR}$ (Allergan, Inc., Irvine, California, USA) was injected in the anterior chamber, it did not result in irreversible endothelial cell damage.[58,59]

Another focus is on improving the bioavailability of ocular drugs through the use of different formulations, such as microspheres[60], nanoparticles[61], liposomes, micelles, and prodrugs. These solutions are promising but do not guarantee higher patient compliance than eye drops.[52,62,63]

Finally, ocular treatments can be improved by better understanding the basic ocular and corneal pharmacokinetics.[43,64-67] Therefore, fundamental research is indispensable and essential for the applied sciences.

In this review, we will cover resorbable devices, oval- and ring-shaped devices, rodshaped devices, punctum plugs, and contact lenses and ocular shields. The characteristics of all these devices are summarized in Table 2-2.

\subsubsection{Resorbable conjunctival devices}

Resorbable drug delivery devices are devices which can be placed in the conjunctival sac and which dissolve and secrete drugs over time. The advantages of resorbable devices are that they are often non-invasive and do not need to be removed. However, most resorbable devices have a limited time of action (typically less than 24 hours) and thus may require frequent administration. [68] Moreover, it is challenging to develop resorbable devices since the complete material and its metabolites should be non-toxic. Other challenges are the prevention of accidental loss of the device, which is not always noticed and the increase in tear production after placement which increases the risk of bulk release of drugs.[68]

The only resorbable conjunctival device which is on the market is Lacrisert ${ }^{\circledR}$ (Aton Pharma, Lawrenceville, New Jersey, USA) (figure 2-2,7). Lacrisert ${ }^{\circledR}$ is a small hydroxypropyl cellulose tablet which has to be placed in the lower conjunctival fornix (Table 2). It slowly dissolves and creates an artificial tear film to treat dry eyes. [69] However, Luchs et al. showed that wearing a Lacrisert ${ }^{\circledR}$ insert can lead to blurred vision which warranted removal of the device in $8.7 \%$ of the participants.[70] Other known adverse effects related to wearing a Lacrisert ${ }^{\circledR}$ insert are ocular discomfort or ocular irritation because of foreign body sensations, stickiness of eyelashes, photophobia, hypersensitivity, eyelid edema, and hyperemia.[70,71]

Another resorbable device called New Ophthalmic Delivery System (NODS ${ }^{\circledR}$ ) was developed by Smith and Nephew Pharmaceuticals Ltd. (Gilston Park, Harlow, 
Essex, UK). The NODS ${ }^{\circledR}$ is made from water-soluble polyvinyl alcohol (PVA) and should be placed in the cul-de-sac of the lower eyelid. The NODS ${ }^{\circledR}$ has been loaded with different drugs e.g. pilocarpine, chloramphenicol and tropicamide.[72]

This device assured an eight-fold increase in drug bioavailability compared to eye drops in healthy volunteers.[73-75] A small clinical trial with twelve volunteers revealed intense miosis in all test subjects as a side effect.[75] In a larger study with twenty-nine volunteers, there were some problems with the detachment of the NODS $^{\circledR}$ from its applicator.[74] Eventually, only the tropicamide loaded NODS ${ }^{\circledR}$ was introduced into the market. Although the price was comparable to that of a tropicamide Minims ${ }^{\circledR}$ (eye drops), the product was not commercially successful since it could only be used for diagnostic purposes.[72] Therefore, many of the benefits, such as absence of preservative, improved bioavailability, and convenience of storage were not relevant.[72]

Another group investigated Gelfoam ${ }^{\circledR}$ discs (Pharmacia \& Upjohn Company LLC, Peapack, New Jersey, USA) as an alternative drug delivery system. The Gelfoam ${ }^{\circledR}$ discs are made of resorbable gelatin and impregnated with insulin for diabetic patients or mydriatic drugs to widen the pupil. The Gelfoam ${ }^{\circledR}$ discs should be placed in the lower conjunctival fornix and have been tested extensively in rabbits and human volunteers.[76-79] Some volunteers (6/20) developed a palpebral conjunctival infection (hyperemia), while other volunteers $(3 / 20)$ developed superficial punctate erosion.[76] No further use of this device was reported.

\subsubsection{Oval- and ring-shaped conjunctival devices}

Several non-resorbable devices shaped as a ring or oval structure have been developed (Table 2-2). These devices are placed under the upper and/or lower eyelid in the conjunctival fornices and use the non-corneal route to distribute drugs. One of the first breakthroughs within this field came from ALZA Corporation (Mountain View, California, USA) (acquired by Johnson \& Johnson, New Brunswick, New Jersey, USA in 2001). ALZA invented an oblong-shaped device (figure 2-2,8), called Ocusert ${ }^{\circledR}$, which consisted of two ethylene-vinyl acetate (EVA) membranes filled with pilocarpine and covered by a ring of titanium dioxide impregnated EVA (Table 2-2).[73] The preferred location to place the Ocusert ${ }^{\circledR}$ was the upper conjunctival sac (figure 2-2,8b) and resulted in one week of drug delivery.[80] Ocusert $^{\circledR}$ came on the market in July 1974 and was available in two doses, the Ocusert ${ }^{\circledR}$ Pilo-20 (release of $20 \mu \mathrm{g} / \mathrm{hr}$ ) and the Ocusert ${ }^{\circledR}$ Pilo-40 (release of $40 \mu \mathrm{g} / \mathrm{hr}$ ). However, the Ocusert ${ }^{\circledR}$ was discontinued because of foreign body sensation, retention issues, difficulty in handling, and only marginal IOP reduction. [73,80-83] Because of these adverse effects and the low efficacy, the acceptance of Ocusert ${ }^{\circledR}$ by the ophthalmic market was low. $[82,84]$ 


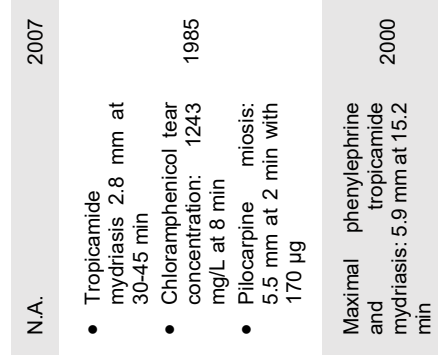

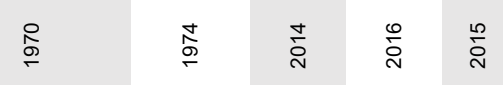

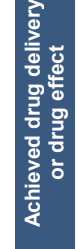

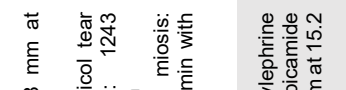

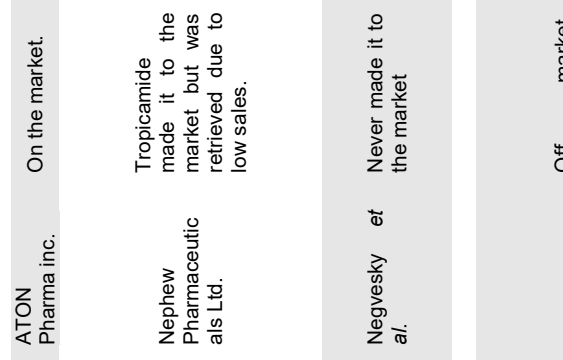

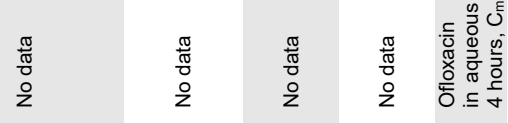

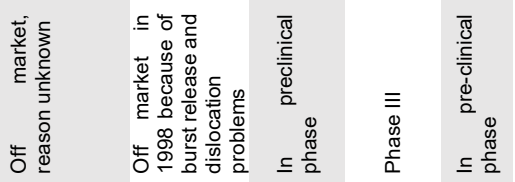

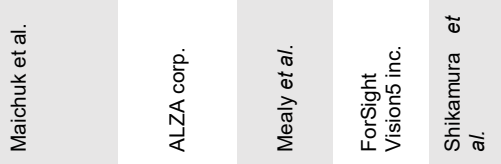

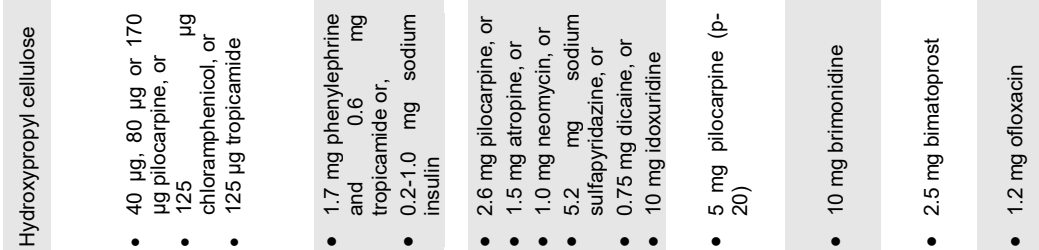

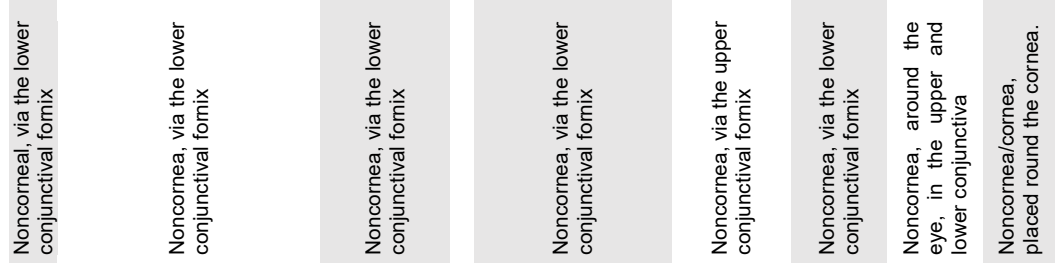

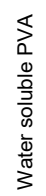

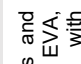

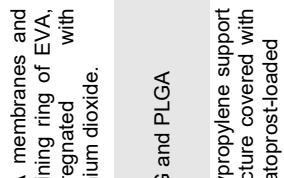

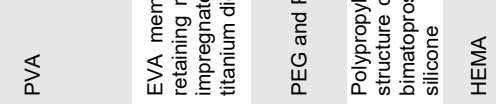

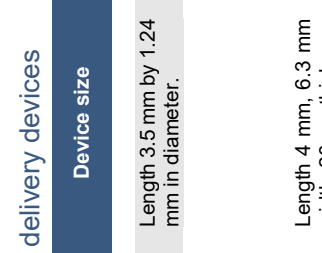

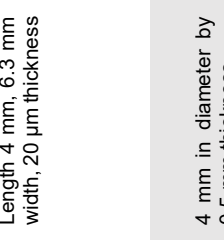

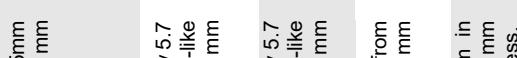

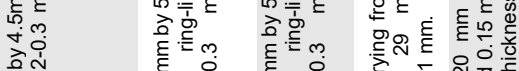
\begin{tabular}{llll} 
& & \\
\hline
\end{tabular}

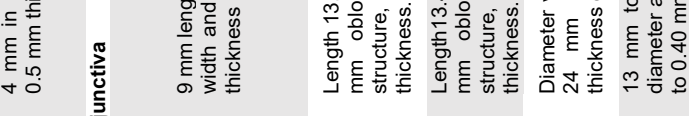
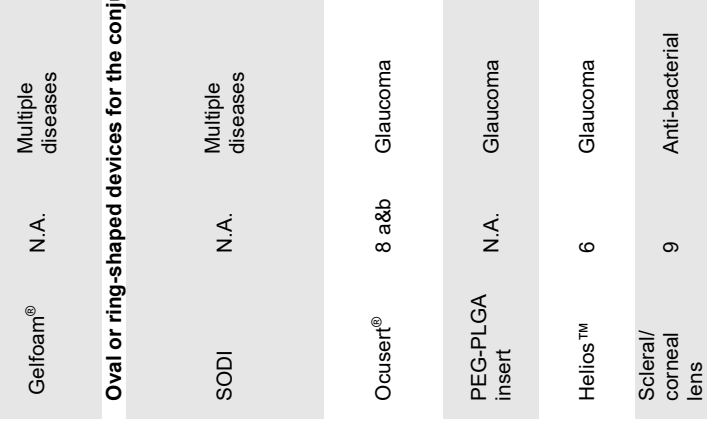


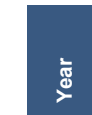

亭

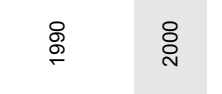

蒙

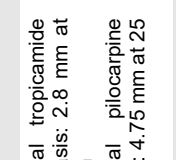

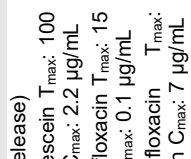

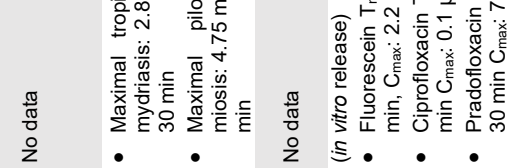

递

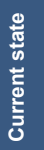

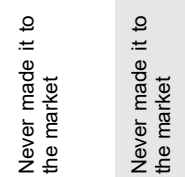

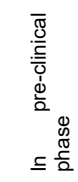

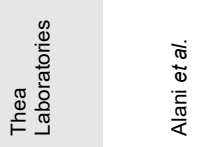

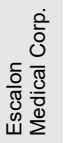

을 요

产号棺

है

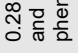

辤

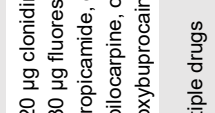

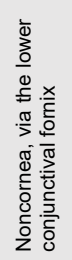

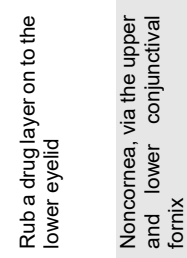

这亭

产

$\frac{\overline{\frac{\pi}{\pi}}}{\frac{\bar{m}}{\frac{\mathrm{m}}{2}}}$

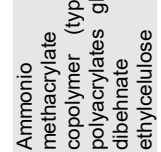

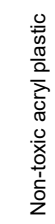

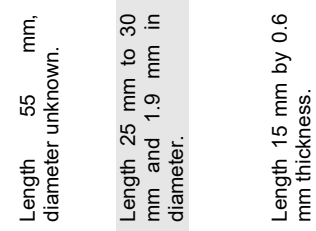

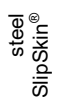

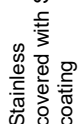

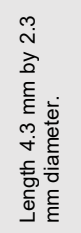

흥

产

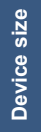

옿

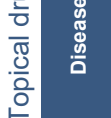

흠

竞

กิ่

$\frac{0}{\frac{0}{0}}$

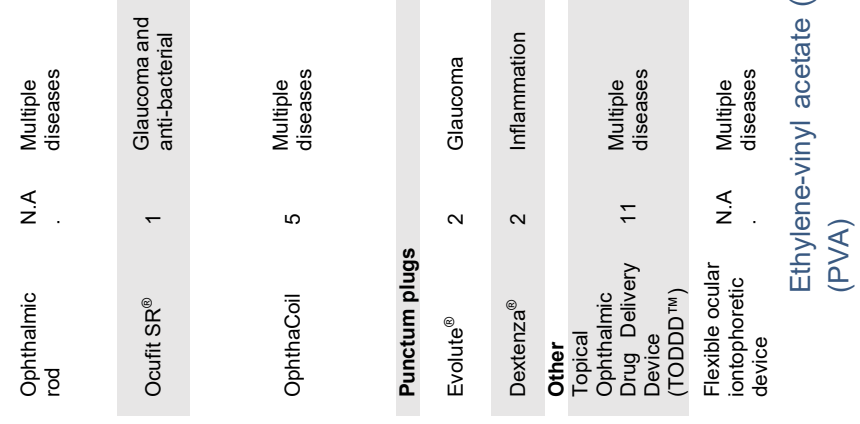


More recently, a polyethylene glycol (PEG) and polylactic-co-glycolic acid (PLGA) elliptical insert was created with a similar size and shape to the Ocusert ${ }^{\circledR}$ ring. An in vitro study showed that the brimonidine tartrate-loaded insert produced a linear drugrelease profile for one month. Further investigation is needed to demonstrate the potential of this drug-eluting device in the treatment of glaucoma.[83]

One particular example of a drug-loaded ring structure was developed by a Japanese research group. They developed a 2-hydroxyethyl methacrylate (HEMA) contact lens with a central hole. The device (loaded with $0.3 \%$ ofloxacin) could deliver ofloxacin to the anterior and even the posterior parts of the eye in rabbits. Drug delivery to the posterior part of the eye was achieved in 15-60 minutes after application of the device, via penetration of the conjunctiva and sclera into the choroid. Drug concentrations in the posterior tissue were more than ten times lower than those in the anterior tissue. However, compared to drug delivery from eye drops and corneal hydrogel lenses, drug concentrations in posterior tissue were ten to forty times higher, respectively.[20]

A ring-shaped device (Helios ${ }^{\mathrm{TM}}$ ) (figure 2-2,6, Table 2-2) was developed by ForSight Vision5 Inc. (Menlo Park, California, USA). This ring (24-29 mm diameter, $1 \mathrm{~mm}$ thickness) consisted of an internal polypropylene support covered with bimatoprostloaded silicone. The Helios ${ }^{\mathrm{TM}}$ ring had to be placed around the eye and can be used for the reduction of the IOP in glaucoma patients.[85,86] A phase II clinical trial $(130$ patients) demonstrated that the Helios ${ }^{\mathrm{TM}}$ ring reduced the IOP $(4-6 \mathrm{mmHg})$ over a six month period. However, this IOP reduction was not significantly different when compared to regular unpreserved timolol $0.5 \%$ ophthalmic solution (Valeant Ophthalmics, Bridgewater, New Jersey, USA) (after 6 months a reduction of $3.25 \pm$ $0.32 \mathrm{mmHg}$ with bimatoprost compared to $4.24 \pm 0.37$ to timolol $0.5 \%$ ophthalmic solution). In addition, the drop-out rate was higher in the patient group with the Helios $^{\mathrm{TM}}$ device (8 versus 2 in the eye drop group).[86] A 13 month safety study (with a 6-month and 7-month interval) showed a safety profile consistent with bimatoprost exposure except for an increased incidence of ocular discharge (mucus). The retention rate after 13 months was $94.7 \%$ suggesting that retention improves as patients gain more experience using the ring.[87]

\subsubsection{Rod-shaped conjunctival devices}

Another group of non-resorbable devices are the rod-shaped devices. These devices should also be placed in the upper or lower conjunctival fornix to deliver drugs via the non-corneal absorption route.

One small rod-shaped device that is available on the European market since 2004 is Mydriasert ${ }^{\circledR}$ (Thea Laboratories, Clermont-Ferrand, France) (figure 2-2,7, Table 22). Mydriasert ${ }^{\circledR}$ is an ethyl cellulose tablet which is loaded with tropicamide and phenylephrine hydrochloride to deliver mydriasis two hours before surgery. 
However, when compared to topical mydriatic eye drops, there was no significant difference in pupil dilatation. In addition, topical mydriatic eye drops dilate the pupil faster (within 15 minutes) compared to the Mydriasert ${ }^{\circledR}$ insert. $[88,89]$ The economic benefits of Mydriasert ${ }^{\circledR}$ were investigated in a cohort of 1763 patients in the U.K. Although an insert is more expensive compared to eye drops ( $£ 4.20$ per insert compared to $£ 0.41$ per vial for tropicamide $1 \%$ and $£ 0.49$ per vial of phenylephrine hydrochloride $10 \%$ ), nurse time could be decreased thereby saving $£ 1.20$ per patient. This resulted in a decrease of $18 \%$ in the total annual costs.[90]

In the early nineties, the ophthalmic rod was developed.[91,92] This rod was intended as a single-dose sterile applicator of drugs in order to avoid the problems of preservation, sterility, cross-infection, and cross-contamination of eye drops. The non-toxic acrylic plastic rod could be loaded on one end with drugs (e.g. tropicamide, oxybuprocaine, fluorescein, or pilocarpine) by dipping the rod into an alcohol-drug solution. After evaporation of the alcohol, the drug-film could be used. The drugs were released in the lower conjunctiva by introducing the tip of the rod in the conjunctival sac and rubbing it against the palpebral conjunctiva of the lower lid. In this way, a small drug-film was created on the conjunctiva which was slowly dissolved by the tear film. The development of the rod was discontinued because of the induced mechanical stress on the tissue, drug preservation issues and the problem of its use in combination with other eye drops.[91,92]

Escalon Medical Corp. (Wayne, Pennsylvania, USA) patented Ocufit SR ${ }^{\circledR}$, a drugeluting rod-shaped ocular device which could be placed in the lower and upper conjunctival fornix (figure 2-2,1, Table 2).[69] The cylindrical rod was made of a silicone elastomer and loaded with drugs for the treatment of glaucoma or with antibiotics.[73,93] Although the placebo device could be retained in the upper fornix of the eye for over two weeks in $70 \%$ of volunteers,[73] the phase I study with the Ocufit $\mathrm{SR}^{\circledR}$ device was discontinued in 2000 because of reallocation of the company's research and development interests.[94]

Finally, our group developed the OphthaCoil (figure 2-2,5, Table 2-2), a coiled stainless steel wire, which is placed in the lower conjunctival sac. The device can be filled with drugs inside its lumen (loaded on microspheres or filaments) or outside on the SlipSkin ${ }^{\circledast}$ coating.[95-100] The OphthaCoil was loaded with pradofloxacin and mydriatic agens (phenylephrine hydrochloride and tropicamide) and tested in Beagle dogs and horses. The pradofloxacin-loaded OphthaCoil resulted in drug delivery concentrations higher than the minimum inhibitory concentration (MIC) and the mydriatic-loaded OphthaCoil resulted in complete dilation one hour after placement which lasted for one to four hours after removal of the OphthaCoil.[101] Although tolerability of the OphthaCoil was excellent, the device was lost overnight in dogs and horses (probably because of the third eyelid, also called the nictitating membrane, which covers and protects the eyes during sleep). This is unlikely to 
occur in humans since humans do not have a third eyelid.[100] In humans (pilot trials), short-term high tolerance and comfort of the device was demonstrated for a period of two hours.[99,101] Currently, new preclinical and clinical trials are being executed in order to further explore the potential of an ocular coil as an ocular drug delivery device for an extended period of time, up to 28 days.

\subsubsection{Punctum plugs}

Other types of ocular devices that show potential to be used as drug delivery devices are punctum plugs (figure 2-2,2, Table 2-2). These small plugs must be placed in the tear duct and were initially invented for patients with keratoconjunctivitis sicca (dry eye syndrome).[102-104] The first punctum plug used as an ocular drug delivery device was already developed in 1974.[105]

In 2012 Mati Therapeutics (Austin, Texas, USA) and QLT Inc. (Vancouver, British Columbia, Canada) started collaborating in the field of punctum plugs.[106-108] They developed a latanoprost punctal plug delivery system (known as L-PPDS or Evolute ${ }^{\circledR}$ ) with a small drug reservoir in the head of the plug. After placement, the plug was able to deliver drugs via the lacrimal system into the tear film and to the tear duct.[109] In 2015, a phase Ilb multicenter trial was started to evaluate the efficacy of the L-PPDS. So far, no results or details have been reported.[110,111] Ocular Therapeutix Inc. (Bedford, Massachusetts, USA) developed Dextenza ${ }^{\circledR}$, a 0.4 mg dexamethasone containing PEG punctum plug (figure 2-2.2, Table 2-2) for the treatment of inflammatory eye conditions up to 30 days after a cataract surgery. $A$ phase II trial $(n=60)$ showed that Dextenza ${ }^{\circledR}$ was effective in stopping itching and providing pain relief after cataract surgery.[112] Another Phase II clinical trial in a group of 28 patients with allergic conjunctivitis (versus 31 patients in the vehicle group) showed improvement of allergic signs and symptoms in a 6 week trial. However, no significant difference in itching and ocular redness was observed between the Dextenza ${ }^{\circledR}$ group and the vehicle group.[113] A phase III trial to demonstrate treatment of ocular itching associated with allergic conjunctivitis was also successful.[110,111] Another phase III trial investigated the use of Dextenza ${ }^{\circledR}$, for the treatment of ocular inflammation and pain after ophthalmic surgery (e.g. cataract). Absence of anterior chamber cells and ocular pain on days 4,14 , and 30 after insertion of the insert was shown.[114] With these results, Ocular Therapeutix announced their intention to file a FDA new drug application (NDA) to bring their product to the market.[111,112,115-117] In July 2017, the FDA rejected Ocular Therapeutix's NDA due to deficiencies in the manufacturing process and analytical testing identified during a pre-NDA approval inspection of an Ocular Therapeutix manufacturing facility.[118] 


\subsubsection{Contact lenses and corneal shields}

Contact lenses and corneal shields have also been investigated for ocular drug delivery (figure 2-2,9). They are able to transport drugs via the corneal route, but must remain transparent in order to prevent vision loss. One potential advantage is that they could simultaneously deliver drugs and enhance vision by correcting the refractive error. After placement of a drug-loaded contact lens on the eye, the drug slowly diffuses into a thin fluid layer between the lens and the cornea, called the preocular or post-lens tear film (POTF), and diffuses slowly through the surrounding tissues (via the cornea, limbus, and conjunctiva) into the anterior segment of the eye.[119,120]

Since 1960, contact lenses and shields have been investigated to deliver drugs to the eye.[121-126] Most of them were made by simply dipping the material (often a hydrogel) into a drug solution.[127] This 'soak and release' approach did not lead to a successful clinical product, mainly because of the short duration of release.[128] Currently, more innovative ways of drug-loading are being explored, for example by molecular imprinting or entrapping of nanoparticles into the polymer structure.[120,124,129-131]

Although drug-loaded contact lenses result in an increased bioavailability of the drug over eye drops, and in silico and animal studies have proven safety and efficacy[120,129,132], drug-eluting contact lenses have not yet reached the market. Contact lenses are associated with an increased risk of contact lens-related corneal damage and infections.[133-135]

The following examples of drug-loaded contact lenses are promising: a latanoprost secreting PLGA contact lens developed by the department of Ophthalmology Massachusetts Eye and Ear Infirmary from Harvard Medical School (Boston, Massachusetts, USA), which was tested in glaucoma induced monkeys[136], a poly$\varepsilon$-lysine (p\&K) hydrogel bandage lens containing amphotericin B for treatment of fungal keratitis which was tested in vitro, and is currently under development by the department of Eye and Vision Science, Institute of Ageing and Chronic Diseases from the University of Liverpool (Liverpool, UK)[137], and a brimonidine eluting thermosensitive hydrogel (consisting of PLGA-PEG-PLGA) with nanoparticles, which was developed by the department of Ophthalmology \& Visual Science and the Department of Pharmacy from the Eye \& ENT Hospital (Shanghai, China). This thermosensitive gel is applied between a soft contact lens and the cornea and was tested in vitro and in animals for its drug-secreting capacity.[138] More contact lens and bandage lens related technologies are reviewed in.[131,139,140]

\subsubsection{Other devices}

Another example of a drug-delivery device with a particular shape has been developed by Amorphex Therapeutics (Dundee Park. Andover, Massachusetts, 
USA) (Table 2-2). The device called TODDD ${ }^{\mathrm{TM}}$ (Topical Ophthalmic Drug Delivery Device) is an 'eight-shaped' (figure 2-2,11) timolol or prostaglandin-containing elastomer ( $20 \mathrm{~mm}$ length, about $8 \mathrm{~mm}$ width, and $1 \mathrm{~mm}$ thickness) which should be placed on the sclera below the upper eyelid of glaucoma patients.[141] In a human trial, $(n=20)$ the timolol-loaded device showed that the IOP was reduced by $16 \%$ to $22 \%$ in glaucoma patients after 6 months.[115]

Finally, it is expected that a significant part of the population will have intraocular lenses (IOLs) implanted in the near future based on aging and extended life expectancies. So far, only few attempts have been made to use IOLs as drug delivery agents to prevent postoperative infection[142-145] and inflammation[144] or posterior capsule opacification, the most frequent complication of cataract surgery.[146,147] As far as we know, this has not resulted in commercial applications.

\subsection{Challenges in pharmacokinetics}

Ocular drug delivery devices have demonstrated improved drug uptake over conventional drug formulations. For example, higher uptake was measured through a corneal ring (13 $\mu \mathrm{g} / \mathrm{g}$ in the cornea and $4 \mu \mathrm{g} / \mathrm{mL}$ in the aqueous humour)[20], as compared to a topical solution $(6.95 \mu \mathrm{g} / \mathrm{g}$ in the cornea and $1.42 \mathrm{ng} / \mathrm{\mu g}$ in the aqueous humor).[148] However, besides on the delivery route, the level of drug uptake can be influenced significantly by a number of factors. Pharmacokinetics of eye drops can be improved by adapting the $\mathrm{pH}$ (affecting the logD7.4)[18] or adding additives to the formulation like frinstens benzalkonium chloride (BAC), that adds antimicrobial properties and enhances corneal permeability.[149] According to some studies, EDTA enhances corneal penetration by chelation of calcium ions involved in opening of tight junctions. [150-153] Other studies however show no effect of EDTA in hydrophilic drugs $[154,155]$ or lipophilic drugs.[153,156,157] Another enhancer for corneal permeability of lipophilic drugs is alpha cyclodextrin, which increases the solubility.[155,158] Enhanced uptake of drugs can also be achieved by esterified compounds which are often more lipophilic.[159] For example, compared to mice instilled with BAC enriched LA, aqueous humour concentrations of LA were five times higher in eyes of mice following treatment with LA and its choline ester (LACE)[1]. Another example of an esterified drug is latanoprost (a prodrug from prostaglandin $\mathrm{F} 2 \mathrm{a}$ ) which increases uveoscleral outflow, thereby lowering the IOP by $20 \%-35 \%$ in patients with open-angle glaucoma.[160] Latanoprost was instilled in rabbits ( $30 \mu \mathrm{L}, 0.005 \%$ solution), which resulted in concentrations of $82 \pm 35 \mathrm{pg} / \mathrm{uL}$ in the aqueous humor ( $T_{\max } 1$ hour), $90 \pm 34 \mathrm{pg} / \mathrm{mg}$ in the conjunctiva ( $T_{\max } 15 \mathrm{~min}$ ), $1,202 \pm 576 \mathrm{pg} / \mathrm{mg}$ in the cornea ( $T_{\max } 15 \mathrm{~min}$ ), and $264 \pm 157 \mathrm{pg} / \mathrm{mg}$ in the ciliary body $\left(T_{\max } 15 \mathrm{~min}\right)$.[161] These concentrations are in line with results obtained in 
monkeys.[160,162] After topical $(50 \mu \mathrm{g} / \mathrm{mL})$ administration in humans, systemic latanoprost bioavailability was $45 \%$ with a maximum concentration $\left(\mathrm{C}_{\max }\right)$ of 53 $\mathrm{pg} / \mathrm{mL}$ after $5 \mathrm{~min}\left(\mathrm{~T}_{\max }\right)$. About $88 \%$ of the available drug was recovered by the kidneys.[160]

Besides additives and chemical modifications, oral supplementation of antioxidants in combination with topical nutraceutical components leads to decreased reactive oxygen species in the retina and lens, and enhances corneal permeability.[4] Intravenous injections of the combretastatin A-4 prodrug (vascular disrupting agent) lead to effective drug concentrations for the prevention of neovascular age-related macular degeneration in galactose-fed dogs[163] and in patients.[164]

Furthermore, pharmacokinetics can be altered due to eye rotations[29,30], ocular diseases[22,31,32], ocular surgery[165] and coating and modification of IOL materials.[142,145,147,166,167]

\subsection{Discussion and future prospectives}

The continuous increase in the number of ocular surgeries, combined with low patient compliance and low drug bioavailability, warrants the development of new drug delivery methods to the eye. Fortunately, progress has been made in recent years. Although conventional drug delivery formulations, such as eye drops and ointments, are easy to use, inexpensive new drug delivery devices should guarantee higher patient compliance and higher drug concentrations at the target site. Furthermore, drug treatments can be improved by combining next generation drug formulations (such as microspheres, nanoparticles and micelles) into the new drug delivery devices and by increasing fundamental knowledge on drug pharmacokinetics.

However, the public domain often lacks crucial information, in particular on achieved drug concentrations and effects in the various intraocular tissues. This may be due to the fact that companies are not keen on sharing proprietary information. Another major reason for the paucity of pharmacokinetic data of intraocular drug concentrations is that there are no non-invasive diagnostic methods to gather these data. Only invasive sampling at the time of surgery can be used or indirect ways of measuring drug effects, e.g. measuring mydriasis when using dilating eye drops or drug delivery systems (see Table 2-2). This diagnostic measurement barrier makes it complex to compare new drug delivery devices to eye drops.

An important consideration with drug delivery devices is the shape of the device, since this is essential in terms of drug capacity, dislocation/retention and comfort. The question of which shape of device is most functional remains unanswered. In 1977 Katz et al. showed in a comparison study with 68 volunteers (128 rod-shaped devices and 127 oval-shaped devices) that rod-shaped devices were better tolerated 
compared to oval-shaped devices. Nevertheless, a number of devices of both shapes were lost after waking up by 'rubbing the sleep out of the eyes'.[168]

The idea of ocular ring structures was already patented in 1979 (U.S. 3,995,635[85]) the Helios ${ }^{\mathrm{TM}}$ ring is visible whilst worn which puts forward the question of whether this is desirable from an aesthetic point of view. Other devices such as the TODDD ${ }^{\mathrm{TM}}$ or the OphthaCoil are less visible since they are covered by the eyelids. Both devices are still in the developmental phase and there is no extensive data on retention and comfort outcome parameters in humans available yet. So far, an animal study showed that the OphthaCoil was not retained in dogs, most likely due to the animal's third eyelid (nictitating membrane). Similarly, dislocation of the Ocusert ${ }^{\circledR}$ was described and was one of the reasons for market retrieval.[73]

Contact lenses seem promising topical drug delivery devices. However, as they need to remain transparent and oxygen permeable[169] drug delivery via contact lenses is challenging. The newest advanced technologies (e.g. nanoparticles[170], micelles[171], and liposomes[172]) will help contact lenses to become another player in the drug delivery field.

Another way to deliver drugs is by a punctum plug, as currently executed by Ocular Therapeutix. During the first patent of the punctum plug, the possibility of ocular drug delivery was already covered (U.S. 3,949,750[105]). Although punctum plugs have shown effective drug delivery up to six weeks[113], they have a limited drug loading capacity due to their small size.

Despite these challenges in drug capacity, potential dislocation and discomfort, we believe that the trend towards using ocular devices for drug delivery is inevitable, as this may take away the daily burden of administration of eye drops. For cataract surgery the concept of "dropless cataract surgery", implying no use of eye drops around the surgical procedure anymore, has recently been introduced.[173] Although the applied dose of drugs is often lower in the medical devices, the continuous and more stable release of drugs seems be more effective and more preferred by the tissues compared to the intervals with higher doses from eye drops.[86] Another advantage of ocular devices is the absence of preservatives and absorption enhancers. It is well known that these molecules (e.g. benzalkonium chloride and EDTA) can have serious side-effects on the cornea and could eventually lead to the development of intolerable discomfort and allergies. Finally, decreased use of homecare for installation of eye drops in the elderly ophthalmic patient population will result in economic benefits.[90]

With respect to the ophthalmic market, today there are only two conjunctival inserts available for 'non-invasive' drug delivery, i.e. Mydriasert ${ }^{\odot}$ and Lacrisert ${ }^{\odot}$. Although the new European Medical Device Regulation (MDR) will require more evidence on the effectiveness of new devices (for which patient compliance is pivotal, see figure 2-2), more inserts are expected to join the market in the following years. The FDA 
has also recognized the need for new drug delivery devices for ocular use. The FDA now accepts first-in-human trials earlier and promised to simplify the approval process of new devices.[174] This provides opportunities for researchers, ophthalmologists and the ophthalmic industry to realize the goal of improved ocular drug delivery. Only by working together (academia, industry, and authorities) and by exploring parallel strategies (new drug delivery devices, enhanced drug formulations, better understanding of the pharmacokinetic properties), the therapeutic effect of drug treatments can be improved.

\subsection{References}

1. Garner, W.H. and Garner, M.H., Protein Disulfide Levels and Lens Elasticity Modulation: Applications for Presbyopia. Invest Ophthalmol Vis Sci, 2016. 57(6): p. 2851-2863.

2. Makley, L.N., McMenimen, K.A., DeVree, B.T., Goldman, J.W., McGlasson, B.N., Rajagopal, P., Dunyak, B.M., McQuade, T.J., Thompson, A.D., Sunahara, R., Klevit, R.E., Andley, U.P., and Gestwicki, J.E., Pharmacological chaperone for alpha-crystallin partially restores transparency in cataract models. Science, 2015. 350(6261): p. 674-677.

3. Zhao, L., Chen, X.J., Zhu, J., Xi, Y.B., Yang, X., Hu, L.D., Ouyang, H., Patel, S.H., Jin, X., Lin, D., Wu, F., Flagg, K., Cai, H., Li, G., Cao, G., Lin, Y., Chen, D., Wen, C., Chung, C., Wang, Y., Qiu, A., Yeh, E., Wang, W., Hu, X., Grob, S., Abagyan, R., Su, Z., Tjondro, H.C., Zhao, X.J., Luo, H., Hou, R., Jefferson, J., Perry, P., Gao, W., Kozak, I., Granet, D., Li, Y., Sun, X., Wang, J., Zhang, L., Liu, Y., Yan, Y.B., and Zhang, K., Lanosterol reverses protein aggregation in cataracts. Nature, 2015. 523(7562): p. 607-611.

4. Kador, P.F., Guo, C., Kawada, H., Randazzo, J., and Blessing, K., Topical nutraceutical Optixcare EH ameliorates experimental ocular oxidative stress in rats. J Ocul Pharmacol Ther, 2014. 30(7): p. 593-602.

5. Pucker, A.D., Ng, S.M., and Nichols, J.J., Over the counter (OTC) artificial tear drops for dry eye syndrome. Cochrane Database Syst Rev, 2016. 2: p. CD009729.

6. Trese, M.G.J., Lewis, A.W., Blachley, T.S., Stein, J.D., and Moroi, S.E., Changing Initial Glaucoma Medical Therapy Increases Healthcare Resource Utilization. J Ocul Pharmacol Ther, 2017. 33(8): p. 591-597.

7. Jonas, J.B., Aung, T., Bourne, R.R., Bron, A.M., Ritch, R., and Panda-Jonas, S., Glaucoma. Lancet, 2017. 390(10108): p. 2183-2193.

8. Lavia, C., Dallorto, L., Maule, M., Ceccarelli, M., and Fea, A.M., Minimally-invasive glaucoma surgeries (MIGS) for open angle glaucoma: A systematic review and meta-analysis. PLoS One, 2017. 12(8): p. e0183142.

9. Jick, S., Lens and Cataract. 2016-2017 ed. Basic and Clinical Science Course, ed. A.A.o. Ophthalmology. Vol. 2016-2017. 2016, San Francisco, Calif.: American Academy of Ophthalmology. 256.

10. Shah, N., Maguire, M.G., Martin, D.F., Shaffer, J., Ying, G.S., Grunwald, J.E., Toth, C.A., Jaffe, G.J., Daniel, E., and Comparison of Age-related Macular Degeneration Treatments Trials Research, G., Angiographic Cystoid Macular Edema and Outcomes in the Comparison of AgeRelated Macular Degeneration Treatments Trials. Ophthalmology, 2016. 123(4): p. 858-864.

11. Wielders, L.H., Schouten, J.S., Aberle, M.R., Lambermont, V.A., van den Biggelaar, F.J., Winkens, B., Simons, R.W., and Nuijts, R.M., Treatment of cystoid macular edema after cataract surgery. J Cataract Refract Surg, 2017. 43(2): p. 276-284.

12. Kim, S.J., Schoenberger, S.D., Thorne, J.E., Ehlers, J.P., Yeh, S., and Bakri, S.J., Topical Nonsteroidal Anti-inflammatory Drugs and Cataract Surgery: A Report by the American Academy of Ophthalmology. Ophthalmology, 2015. 122(11): p. 2159-2168.

13. Kim, S.J., Patel, S.N., and Sternberg, P., Jr., Routine Use of Nonsteroidal Anti-inflammatory Drugs with Corticosteroids in Cataract Surgery: Beneficial or Redundant? Ophthalmology, 2016. 123(3): p. 444-446.

14. Wielders, L.H., Lambermont, V.A., Schouten, J.S., van den Biggelaar, F.J., Worthy, G., Simons, R.W., Winkens, B., and Nuijts, R.M., Prevention of Cystoid Macular Edema After Cataract 
Surgery in Nondiabetic and Diabetic Patients: A Systematic Review and Meta-Analysis. Am J Ophthalmol, 2015. 160(5): p. 968-981 e933.

15. Kessel, L., Tendal, B., Jorgensen, K.J., Erngaard, D., Flesner, P., Andresen, J.L., and Hjortdal, J., Post-cataract prevention of inflammation and macular edema by steroid and nonsteroidal anti-inflammatory eye drops: a systematic review. Ophthalmology, 2014. 121(10): p. 19151924.

16. Cantor, L.B., Cioffi, G.A., and Rapuano, C.J., Glaucoma. 2014-2015. ed. Basic and clinical science course, ed. A.A.o. Ophthalmology. Vol. 2014-2015. 2014, San Francisco, Calif.: American Academy of Ophthalmology. i-263.

17. Vellonen, K.S., Soini, E.M., Del Amo, E.M., and Urtti, A., Prediction of Ocular Drug Distribution from Systemic Blood Circulation. Mol Pharm, 2016. 13(9): p. 2906-2911.

18. Del Amo, E.M., Rimpela, A.K., Heikkinen, E., Kari, O.K., Ramsay, E., Lajunen, T., Schmitt, M., Pelkonen, L., Bhattacharya, M., Richardson, D., Subrizi, A., Turunen, T., Reinisalo, M., Itkonen, J., Toropainen, E., Casteleijn, M., Kidron, H., Antopolsky, M., Vellonen, K.S., Ruponen, M., and Urtti, A., Pharmacokinetic aspects of retinal drug delivery. Prog Retin Eye Res, 2017. 57: p. 134-185.

19. Edward, A. and Prausnitz, M.R., Predicted permeability of the cornea to topical drugs. Pharm Res, 2001. 18(11): p. 1497-1508.

20. Shikamura, Y., Yamazaki, Y., Matsunaga, T., Sato, T., Ohtori, A., and Tojo, K., Hydrogel Ring for Topical Drug Delivery to the Ocular Posterior Segment. Curr Eye Res, 2016. 41(5): p. 653661.

21. Zhang, W., Prausnitz, M.R., and Edwards, A., Model of transient drug diffusion across cornea. J Control Release, 2004. 99(2): p. 241-258.

22. Ho, L.C., Conner, I.P., Do, C.W., Kim, S.G., Wu, E.X., Wollstein, G., Schuman, J.S., and Chan, K.C., In vivo assessment of aqueous humor dynamics upon chronic ocular hypertension and hypotensive drug treatment using gadolinium-enhanced MRI. Invest Ophthalmol Vis Sci, 2014. 55(6): p. 3747-3757.

23. Agrahari, V., Mandal, A., Agrahari, V., Trinh, H.M., Joseph, M., Ray, A., Hadji, H., Mitra, R., Pal, D., and Mitra, A.K., A comprehensive insight on ocular pharmacokinetics. Drug Deliv Transl Res, 2016. 6(6): p. 735-754.

24. Pelkonen, L., Tengvall-Unadike, U., Ruponen, M., Kidron, H., Del Amo, E.M., Reinisalo, M., and Urtti, A., Melanin binding study of clinical drugs with cassette dosing and rapid equilibrium dialysis inserts. Eur J Pharm Sci, 2017. 109: p. 162-168.

25. Del Amo, E.M. and Urtti, A., Rabbit as an animal model for intravitreal pharmacokinetics: Clinical predictability and quality of the published data. Exp Eye Res, 2015. 137: p. 111-124.

26. Goel, M., Picciani, R.G., Lee, R.K., and Bhattacharya, S.K., Aqueous humor dynamics: a review. Open Ophthalmol J, 2010. 4: p. 52-59.

27. Carreon, T.A., Edwards, G., Wang, H., and Bhattacharya, S.K., Segmental outflow of aqueous humor in mouse and human. Exp Eye Res, 2017. 158: p. 59-66.

28. Loewen, R.T., Brown, E.N., Roy, P., Schuman, J.S., Sigal, I.A., and Loewen, N.A., Regionally Discrete Aqueous Humor Outflow Quantification Using Fluorescein Canalograms. PLoS One, 2016. 11(3): p. e0151754.

29. Stocchino, A., Repetto, R., and Cafferata, C., Eye rotation induced dynamics of a Newtonian fluid within the vitreous cavity: the effect of the chamber shape. Phys Med Biol, 2007. 52(7): p. 2021-2034.

30. Bonfiglio, A., Lagazzo, A., Repetto, R., and Stocchino, A., An experimental model of vitreous motion induced by eye rotations. Eye Vis (Lond), 2015. 2: p. 10.

31. Guo, T., Sampathkumar, S., Fan, S., Morris, N., Wang, F., and Toris, C.B., Aqueous humour dynamics and biometrics in the ageing Chinese eye. $\mathrm{Br} \mathrm{J}$ Ophthalmol, 2017. 101(9): p. 12901296.

32. Li, J., Lan, B., Li, X., Sun, S., Lu, P., and Cheng, L., Effect of intraocular pressure (IOP) and choroidal circulation on controlled episcleral drug delivery to retina/vitreous. J Control Release, 2016. 243: p. 78-85.

33. Ambati, J., Canakis, C.S., Miller, J.W., Gragoudas, E.S., Edwards, A., Weissgold, D.J., Kim, I., Delori, F.C., and Adamis, A.P., Diffusion of high molecular weight compounds through sclera. Invest Ophthalmol Vis Sci, 2000. 41(5): p. 1181-1185.

34. Hughes, P.M., Olejnik, O., Chang-Lin, J.E., and Wilson, C.G., Topical and systemic drug delivery to the posterior segments. Adv Drug Deliv Rev, 2005. 57(14): p. 2010-2032. 
35. Bauer, N.J., Motamedi, M., Wicksted, J.P., March, W.F., Webers, C.A., and Hendrikse, F., Noninvasive assessment of ocular pharmacokinetics using Confocal Raman Spectroscopy. J Ocul Pharmacol Ther, 1999. 15(2): p. 123-134.

36. Lee, S.J., Kim, S.J., Kim, E.S., Geroski, D.H., McCarey, B.E., and Edelhauser, H.F., Transscleral permeability of Oregon green 488. J Ocul Pharmacol Ther, 2008. 24(6): p. 579-586.

37. Ahmed, I. and Patton, T.F., Importance of the noncorneal absorption route in topical ophthalmic drug delivery. Invest Ophthalmol Vis Sci, 1985. 26(4): p. 584-587.

38. Kim, H., Robinson, M.R., Lizak, M.J., Tansey, G., Lutz, R.J., Yuan, P., Wang, N.S., and Csaky, K.G., Controlled drug release from an ocular implant: an evaluation using dynamic threedimensional magnetic resonance imaging. Invest Ophthalmol Vis Sci, 2004. 45(8): p. 27222731.

39. Kidron, H., Del Amo, E.M., Vellonen, K.S., and Urtti, A., Prediction of the vitreal half-life of small molecular drug-like compounds. Pharm Res, 2012. 29(12): p. 3302-3311.

40. Lang, J.C., Ocular Drug-Delivery Conventional Ocular Formulations. Advanced Drug Delivery Reviews, 1995. 16(1): p. 39-43.

41. Farkouh, A., Frigo, P., and Czejka, M., Systemic side effects of eye drops: a pharmacokinetic perspective. Clin Ophthalmol, 2016. 10: p. 2433-2441.

42. Palmer, R.M. and Kaufman, H.E., Tear film, pharmacology of eye drops, and toxicity. Curr Opin Ophthalmol, 1995. 6(4): p. 11-16.

43. Urtti, A. and Salminen, L., Minimizing systemic absorption of topically administered ophthalmic drugs. Surv Ophthalmol, 1993. 37(6): p. 435-456.

44. Lee, S.B., Geroski, D.H., Prausnitz, M.R., and Edelhauser, H.F., Drug delivery through the sclera: effects of thickness, hydration, and sustained release systems. Exp Eye Res, 2004. 78(3): p. 599-607.

45. Linn, M.L. and Jones, L.T., Rate of lacrimal excretion of ophthalmic vehicles. Am J Ophthalmol, 1968. 65(1): p. 76-78.

46. Hermann, M.M., Ustundag, C., and Diestelhorst, M., Electronic compliance monitoring of topical treatment after ophthalmic surgery. Int Ophthalmol, 2010. 30(4): p. 385-390.

47. Salyani, A. and Birt, C., Evaluation of an eye drop guide to aid self-administration by patients experienced with topical use of glaucoma medication. Can J Ophthalmol, 2005. 40(2): p. 170174.

48. Nordmann, J.P., Baudouin, C., Renard, J.P., Denis, P., Lafuma, A., Laurendeau, C., Jeanbat, V., and Berdeaux, G., Measurement of treatment compliance using a medical device for glaucoma patients associated with intraocular pressure control: a survey. Clin Ophthalmol, 2010. 4: p. 731-739.

49. Eaton, A.M., Gordon, G.M., Konowal, A., Allen, A., Allen, M., Sgarlata, A., Gao, G., Wafapoor, H., and Avery, R.L., A novel eye drop application monitor to assess patient compliance with a prescribed regimen: a pilot study. Eye (Lond), 2015. 29(10): p. 1383-1391.

50. Newman-Casey, P.A., Robin, A.L., Blachley, T., Farris, K., Heisler, M., Resnicow, K., and Lee, P.P., The Most Common Barriers to Glaucoma Medication Adherence: A Cross-Sectional Survey. Ophthalmology, 2015. 122(7): p. 1308-1316.

51. Mohindroo, C., Ichhpujani, P., and Kumar, S., How 'Drug Aware' are our Glaucoma Patients? J Curr Glaucoma Pract, 2015. 9(2): p. 33-37.

52. Gaudana, R., Ananthula, H.K., Parenky, A., and Mitra, A.K., Ocular drug delivery. AAPS J, 2010. 12(3): p. 348-360.

53. Edman, P., Biopharmaceutics of ocular drug delivery. Pharmacol toxicol. 1993, Boca Raton, Fla.: CRC Press. xi, 202 p.

54. Del Amo, E.M. and Urtti, A., Current and future ophthalmic drug delivery systems. A shift to the posterior segment. Drug Discov Today, 2008. 13(3-4): p. 135-143.

55. Maichuk, Y.F., Letter: Soluble ophthalmic drug inserts. Lancet, 1975. 1(7899): p. 173.

56. Maichuk, Y.F., Editorial: Ophthalmic drug inserts. Invest Ophthalmol, 1975. 14(2): p. 87-90.

57. Bourne, W.M., Biology of the corneal endothelium in health and disease. Eye (Lond), 2003. 17(8): p. 912-918.

58. Tan, D.T., Chee, S.P., Lim, L., and Lim, A.S., Randomized clinical trial of a new dexamethasone delivery system (Surodex) for treatment of post-cataract surgery inflammation. Ophthalmology, 1999. 106(2): p. 223-231.

59. Tan, D.T., Chee, S.P., Lim, L., Theng, J., and Van Ede, M., Randomized clinical trial of Surodex steroid drug delivery system for cataract surgery: anterior versus posterior placement of two Surodex in the eye. Ophthalmology, 2001. 108(12): p. 2172-2181. 
60. Fernandez-Sanchez, L., Bravo-Osuna, I., Lax, P., Arranz-Romera, A., Maneu, V., EstebanPerez, S., Pinilla, I., Puebla-Gonzalez, M.D.M., Herrero-Vanrell, R., and Cuenca, N., Controlled delivery of tauroursodeoxycholic acid from biodegradable microspheres slows retinal degeneration and vision loss in P23H rats. PLoS One, 2017. 12(5): p. e0177998.

61. Chiang, B., Venugopal, N., Edelhauser, H.F., and Prausnitz, M.R., Distribution of particles, small molecules and polymeric formulation excipients in the suprachoroidal space after microneedle injection. Exp Eye Res, 2016. 153: p. 101-109.

62. Cholkar, K., Patel, S.P., Vadlapudi, A.D., and Mitra, A.K., Novel strategies for anterior segment ocular drug delivery. J Ocul Pharmacol Ther, 2013. 29(2): p. 106-123.

63. Kalita, D., Shome, D., Jain, V.G., Chadha, K., and Bellare, J.R., In vivo intraocular distribution and safety of periocular nanoparticle carboplatin for treatment of advanced retinoblastoma in humans. Am J Ophthalmol, 2014. 157(5): p. 1109-1115.

64. Ghate, D. and Edelhauser, H.F., Ocular drug delivery. Expert Opin Drug Deliv, 2006. 3(2): p. 275-287.

65. Shirasaki, Y., Molecular design for enhancement of ocular penetration. J Pharm Sci, 2008. 97(7): p. 2462-2496.

66. Mannermaa, E., Vellonen, K.S., and Urtti, A., Drug transport in corneal epithelium and bloodretina barrier: emerging role of transporters in ocular pharmacokinetics. Adv Drug Deliv Rev, 2006. 58(11): p. 1136-1163.

67. Chun, D., Shapiro, A., and Abelson, M., Ocular pharmacokinetics, in Principles and practice of ophthalmology, D.M. Albert and F.A. Jakobiec, Editors. 2008, W.B. Saunders,: Philadelphia, $\mathrm{Pa}$.

68. Calles, J., Bermudez, J., Vallés, E., Allemandi, D., and Palma, S., Polymers in Ophthalmology, in Advanced Polymers in Medicine, F. Puoci, Editor. 2015, Springer,: Cham. p. 147-176.

69. Rajasekaran, A., Ardul Kumaran, K., Karthika, K., and Padma preetha, J., A Comparative review on conventional and advanced ocular drug delivery formulations. Int J Pharm Tech Res, 2010. 2(4): p. 668-674.

70. Luchs, J.I., Nelinson, D.S., Macy, J.I., and Group, L.A.C.S., Efficacy of hydroxypropyl cellulose ophthalmic inserts (LACRISERT) in subsets of patients with dry eye syndrome: findings from a patient registry. Cornea, 2010. 29(12): p. 1417-1427.

71. McDonald, M., D'Aversa, G., Perry, H.D., Wittpenn, J.R., and Nelinson, D.S., Correlating patient-reported response to hydroxypropyl cellulose ophthalmic insert (LACRISERT(R)) therapy with clinical outcomes: tools for predicting response. Curr Eye Res, 2010. 35(10): p. 880-887.

72. Sesha Rao, L., Rathbone, M.J., Hadgraft, J., and Roberts, M.S., Modified-release drug delivery technology, in Drugs and the pharmaceutical sciences. 2003, Marcel Dekker: New York. p. 329340 .

73. Saettone, M.F. and Salminen, L., Ocular Inserts for Topical Delivery. Advanced drug delivery reviews, 1995. 16(1): p. 95-106.

74. Diestelhorst, M. and Krieglstein, G.K., The ocular tolerability of a new ophthalmic drug delivery system (NODS). Int Ophthalmol, 1994. 18(1): p. 1-4.

75. Greaves, J.L., Wilson, C.G., Birmingham, A.T., Richardson, M.C., and Bentley, P.H., Scintigraphic studies on the corneal residence of a New Ophthalmic Delivery System (NODS): rate of clearance of a soluble marker in relation to duration of pharmacological action of pilocarpine. Br J Clin Pharmacol, 1992. 33(6): p. 603-609.

76. Negvesky, G.J., Butrus, S.I., Abifarah, H.A., Lee, Y.C., and Yalkowsky, S.H., Ocular gelfoam disc-applicator for pupillary dilation in humans. J Ocul Pharmacol Ther, 2000. 16(4): p. 311315.

77. Lee, Y.C., Millard, J., Negvesky, G.J., Butrus, S.I., and Yalkowsky, S.H., Formulation and in vivo evaluation of ocular insert containing phenylephrine and tropicamide. Int J Pharm, 1999. 182(1): p. 121-126.

78. Lee, Y.C., Simamora, P., Pinsuwan, S., and Yalkowsky, S.H., Review on the systemic delivery of insulin via the ocular route. Int J Pharm, 2002. 233(1-2): p. 1-18.

79. Lee, Y.C., Simamora, P., and Yalkowsky, S.H., Systemic delivery of insulin via an enhancerfree ocular device. J Pharm Sci, 1997. 86(12): p. 1361-1364.

80. Quigley, H.A., Pollack, I.P., and Harbin, T.S., Jr., Pilocarpine ocuserts. Long-term clinical trials and selected pharmacodynamics. Arch Ophthalmol, 1975. 93(9): p. 771-775.

81. Kushnick, H., Liebmann, J.M., and Ritch, R., Systemic pilocarpine toxicity from Ocusert leakage. Arch Ophthalmol, 1996. 114(11): p. 1432.

82. Langer, R., Implantable controlled release systems. Pharmacol Ther, 1983. 21(1): p. 35-51. 
83. Mealy, J.E., Fedorchak, M.V., and Little, S.R., In vitro characterization of a controlled-release ocular insert for delivery of brimonidine tartrate. Acta Biomater, 2014. 10(1): p. 87-93.

84. McGhee, C.N., Pharmacokinetics of ophthalmic corticosteroids. Br J Ophthalmol, 1992. 76(11): p. 681-684.

85. Higuchi, T., Hussain, A.A., and Shell, J.W., Ocular insert, A. Corporation, Editor. 1976, Google Patents: USA. p. 6.

86. Brandt, J.D., Sall, K., DuBiner, H., Benza, R., Alster, Y., Walker, G., Semba, C.P., and Collaborators, Six-Month Intraocular Pressure Reduction with a Topical Bimatoprost Ocular Insert: Results of a Phase II Randomized Controlled Study. Ophthalmology, 2016. 123(8): p. 1685-1694.

87. Brandt, J.D., DuBiner, H.B., Benza, R., Sall, K.N., Walker, G.A., Semba, C.P., and Collaborators, Long-term Safety and Efficacy of a Sustained-Release Bimatoprost Ocular Ring. Ophthalmology, 2017. 124(10): p. 1565-1566.

88. Torron, C., Calvo, P., Ruiz-Moreno, O., Lecinena, J., and Perez-Inigo, A., Use of a new ocular insert versus conventional mydriasis in cataract surgery. Biomed Res Int, 2013. 2013: p. 849349.

89. Cagini, C., Caricato, A., Tosi, G., Pascale, A., Cesari, C., and Fiore, T., Evaluation of the efficacy and safety of the ophthalmic insert Mydriasert in patients undergoing retinal angiography. Eur J Ophthalmol, 2014. 24(5): p. 728-734.

90. Shah, A., Johal, S., and Lee, N., Mydriasert pupillary dilation for cataract surgery: an economic and clinical study. BMC Ophthalmol, 2015. 15: p. 56.

91. Alani, S.D., The ophthalmic rod: a new ophthalmic drug delivery system I. Graefes Arch Clin Exp Ophthalmol, 1990. 228(4): p. 297-301.

92. Alani, S.D. and Hammerstein, W., The ophthalmic rod--a new drug-delivery system II. Graefes Arch Clin Exp Ophthalmol, 1990. 228(4): p. 302-304.

93. Darougar, S., Ocular insert for the fornix. 1992, Nies, Kurz, Bergert \& Tamburro: USA. p. 11.

94. Newswire., P. Escalon(R) Medical Corp. Acquires Sonomed Discontinues Clinical Trial of Ocufit $S R(R)$, http://www.prnewswire.com/news-releases/escalonr-medical-corp-acquires-sonomeddiscontinues-clinical-trial-of-ocufit-srr-72104557.html (accessed: 14 July 2016), online. pr newswire 2000 January 18, 2000 [cited 2016 14-07-2016].

95. Dias, A.J.A.A., Koole, L.H., and Pijls, R.T., Coiled wire for the controlled release of drugs to the eye. 2009, Google Patents: USA.

96. Duxfield, L., Sultana, R., Wang, R., Englebretsen, V., Deo, S., Rupenthal, I.D., and Al-Kassas, R., Ocular delivery systems for topical application of anti-infective agents. Drug Dev Ind Pharm, 2016. 42(1): p. 1-11.

97. Pijls, R.T., Cruysberg, L.P., Nuijts, R.M., Dias, A.A., and Koole, L.H., Capacity and tolerance of a new device for ocular drug delivery. Int J Pharm, 2007. 341(1-2): p. 152-161.

98. Pijls, R.T., Hanssen, H.H., Nuijts, R.M., Daube, G.W., and Koole, L.H., In vivo tolerance and kinetics of a novel ocular drug delivery device. J Control Release, 2006. 116(2): p. e47-49.

99. Pijls, R.T., Hanssen, H.H., Nuijts, R.M., and Koole, L.H., Flexible coils with a drug-releasing hydrophilic coating: a new platform for controlled delivery of drugs to the eye? Biomed Mater Eng, 2004. 14(4): p. 383-393.

100. Pijls, R.T., Sonderkamp, T., Daube, G.W., Krebber, R., Hanssen, H.H., Nuijts, R.M., and Koole, L.H., Studies on a new device for drug delivery to the eye. Eur J Pharm Biopharm, 2005. 59(2): p. 283-288.

101. Pijls, R.T., The OphthaCoil, a new vehicle for the delivery of drugs to the eye, in Ophthalmology. 2007, Wöhrmann Print Service: Maastricht. p. 159.

102. Driscoll, A. and Blizzard, C., Toxicity and Pharmacokinetics of Sustained-Release Dexamethasone in Beagle Dogs. Adv Ther, 2016. 33(1): p. 58-67.

103. Yellepeddi, V.K., Sheshala, R., McMillan, H., Gujral, C., Jones, D., and Raghu Raj Singh, T., Punctal plug: a medical device to treat dry eye syndrome and for sustained drug delivery to the eye. Drug Discov Today, 2015. 20(7): p. 884-889.

104. Chee, S.P., Moxifloxacin punctum plug for sustained drug delivery. J Ocul Pharmacol Ther, 2012. 28(4): p. 340-349.

105. Freeman, J.M., Punctum plug and method for treating keratoconjunctivitis sicca (dry eye) and other ophthalmic aliments using same. 1976, Google Patents: USA. p. 7.

106. Novelion. QLT announces exclusive option agreement for potential sale of punctal plug delivery system, http://ir.novelion.com/releasedetail.cfm?releaseid=995645, (accessed: 24 May 2016), online. 2012 [cited 2016 May]. 
107. Lazar, E., Treatment medium delivery device and methods for delivery of such treatment mediums to the eye using such a delivery device. 2011, QLT inc. : USA. p. 15.

108. Odrich, S., Drug delivery via punctal plug. 2005, Google Patents: USA. p. 5.

109. Muller, C. and Utkhede, D., Therapy without Drops: A Reality. ONdrugDel, 2016(63): p. 36.

110. Business wire. Mati Therapeutics Continues to Expand Patent Portfolio, http://www.businesswire.com/news/home/20150506006399/en/Mati-TherapeuticsE\#.VVu_qJNVhBc, (accessed 28 June 2016), online. 2015 May 62015 [cited 201628 july].

111. Business wire. Ocular Therapeutix ${ }^{\mathrm{TM}}$ Announces Topline Results of Phase 3 Clinical Trial for DEXTENZA TM for the Treatment of Allergic Conjunctivitis, http://www.businesswire.com/news/home/20151022006612/en/OcularTherapeutix\%E2\%84\% A2-Announces-Topline-Results-Phase-3, (accessed 8 June 2016), online. 2015 [cited 20168 June].

112. Walters, T., Endl, M., Elmer, T.R., Levenson, J., Majmudar, P., and Masket, S., Sustainedrelease dexamethasone for the treatment of ocular inflammation and pain after cataract surgery. J Cataract Refract Surg, 2015. 41(10): p. 2049-2059.

113. Torkildsen, G., Abelson, M.B., Gomes, P.J., McLaurin, E., Potts, S.L., and Mah, F.S., VehicleControlled, Phase 2 Clinical Trial of a Sustained-Release Dexamethasone Intracanalicular Insert in a Chronic Allergen Challenge Model. J Ocul Pharmacol Ther, 2017. 33(2): p. 79-90.

114. Business wire. Ocular Therapeutix ${ }^{\mathrm{TM}}$ Announces Additional Successful Results for Phase 3 Clinical Trial of DEXTENZA ${ }^{\mathrm{TM}}$, http://www.businesswire.com/news/home/20170104005445/en/Ocular-

Therapeutix\%E2\%84\%A2-Announces-Additional-Successful-Results-Phase, (accessed: 9 January 2017), online. 2017.

115. Bethke, W. New Frontiers in Sustained Release: An update on the most viable strategies for releasing glaucoma drugs over time, https://www.reviewofophthalmology.com/article/newfrontiers-in-sustained-release, (accessed 28 June 2016), online. 2015 [cited 2016 July 28].

116. Cheema, A., Chang, R.T., Shrivastava, A., and Singh, K., Update on the Medical Treatment of Primary Open-Angle Glaucoma. Asia Pac J Ophthalmol (Phila), 2016. 5(1): p. 51-58.

117. Business wire. Ocular Therapeutix ${ }^{\mathrm{TM}}$ Announces Additional Successful Results for Phase 3 Clinical Trial of DEXTENZA TM, http://www.businesswire.com/news/home/20170104005445/en/Ocular-

Therapeutix\%E2\%84\%A2-Announces-Additional-Successful-Results-Phase, (accessed: 9 January 2017), online. 2017 January 4th 2017 [cited 2017 9th of January]; Available from: http://www.businesswire.com/news/home/20170104005445/en/Ocular-

Therapeutix\%E2\%84\%A2-Announces-Additional-Successful-Results-Phase.

118. EyeWorld, FDA rejects NDA for Ocular Therapeutix's dexamethasone insert, http://campaign.r20.constantcontact.com/render?m=1108302654257\&ca=e9d64b47-5ca4-

46c3-b104-e3d4447adfb0 (accessed: 14 July 2017), online. EyeWorld wkly Update, 2017. 23(24).

119. Creech, J.L., Chauhan, A., and Radke, C.J., Dispersive mixing in the posterior tear film under a soft contact lens. Industrial \& Engineering Chemistry Research, 2001. 40(14): p. 3015-3026.

120. Li, C.C. and Chauhan, A., Modeling ophthalmic drug delivery by soaked contact lenses. Industrial \& Engineering Chemistry Research, 2006. 45(10): p. 3718-3734.

121. Willoughby, C.E., Batterbury, M., and Kaye, S.B., Collagen corneal shields. Surv Ophthalmol, 2002. 47(2): p. 174-182.

122. Tian, X., Iwatsu, M., and Kanai, A., Disposable 1-day Acuvue contact lenses for the delivery of lomefloxacin to rabbits' eyes. CLAO J, 2001. 27(4): p. 212-215.

123. Tian, X., Iwatsu, M., Sado, K., and Kanai, A., Studies on the uptake and release of fluoroquinolones by disposable contact lenses. CLAO J, 2001. 27(4): p. 216-220.

124. Dixon, P., Shafor, C., Gause, S., Hsu, K.H., Powell, K.C., and Chauhan, A., Therapeutic contact lenses: a patent review. Expert Opin Ther Pat, 2015. 25(10): p. 1117-1129.

125. Jessen, G.N., Contact Lenses as a Therapeutic Device. Am J Optom Arch Am Acad Optom, 1964. 41: p. 429-435.

126. Wichterle, O. and Lim, D., Hydrophilic Gels for Biological Use. Nature, 1960. 185(4706): p. 117118.

127. Schultz, C.L. and Morck, D.W., Contact lenses as a drug delivery device for epidermal growth factor in the treatment of ocular wounds. Clin Exp Optom, 2010. 93(2): p. 61-65.

128. Bengani, L.C., Hsu, K.H., Gause, S., and Chauhan, A., Contact lenses as a platform for ocular drug delivery. Expert Opin Drug Deliv, 2013. 10(11): p. 1483-1496. 
129. Ali, M., Horikawa, S., Venkatesh, S., Saha, J., Hong, J.W., and Byrne, M.E., Zero-order therapeutic release from imprinted hydrogel contact lenses within in vitro physiological ocular tear flow. J Control Release, 2007. 124(3): p. 154-162.

130. Prakash, M. and Dhesingh, R.S., Nanoparticle Modified Drug Loaded Biodegradable Polymeric Contact Lenses for Sustainable Ocular Drug Delivery. Curr Drug Deliv, 2017. 14(4): p. 555-565.

131. Maulvi, F.A., Soni, T.G., and Shah, D.O., A review on therapeutic contact lenses for ocular drug delivery. Drug Deliv, 2016. 23(8): p. 3017-3026.

132. Gause, S., Hsu, K.H., Shafor, C., Dixon, P., Powell, K.C., and Chauhan, A., Mechanistic modeling of ophthalmic drug delivery to the anterior chamber by eye drops and contact lenses. Adv Colloid Interface Sci, 2016. 233: p. 139-154.

133. Richdale, K., Lam, D.Y., Wagner, H., Zimmerman, A.B., Kinoshita, B.T., Chalmers, R., Sorbara, L., Szczotka-Flynn, L., Govindarajulu, U., and Mitchell, G.L., Case-Control Pilot Study of Soft Contact Lens Wearers With Corneal Infiltrative Events and Healthy Controls. Invest Ophthalmol Vis Sci, 2016. 57(1): p. 47-55.

134. Konda, N., Motukupally, S.R., Garg, P., Sharma, S., Ali, M.H., and Willcox, M.D., Microbial analyses of contact lens-associated microbial keratitis. Optom Vis Sci, 2014. 91(1): p. 47-53.

135. Chalmers, R.L. and Gleason, W., Overview of contact lens postmarket surveillance in the United States: system and recent study results. Eye Contact Lens, 2013. 39(1): p. 109-114.

136. Ciolino, J.B., Ross, A.E., Tulsan, R., Watts, A.C., Wang, R.F., Zurakowski, D., Serle, J.B., and Kohane, D.S., Latanoprost-Eluting Contact Lenses in Glaucomatous Monkeys. Ophthalmology, 2016. 123(10): p. 2085-2092.

137. Gallagher, A.G., McLean, K., Stewart, R.M.K., Wellings, D.A., Allison, H.E., and Williams, R.L., Development of a Poly-epsilon-Lysine Contact Lens as a Drug Delivery Device for the Treatment of Fungal Keratitis. Invest Ophthalmol Vis Sci, 2017. 58(11): p. 4499-4505.

138. Sun, J., Lei, Y., Dai, Z., Liu, X., Huang, T., Wu, J., Xu, Z.P., and Sun, X., Sustained Release of Brimonidine from a New Composite Drug Delivery System for Treatment of Glaucoma. ACS Appl Mater Interfaces, 2017. 9(9): p. 7990-7999.

139. Zidan, G., Rupenthal, I.D., Greene, C., and Seyfoddin, A., Medicated ocular bandages and corneal health: potential excipients and active pharmaceutical ingredients. Pharm Dev Technol, 2018. 23(3): p. 255-260.

140. Papas, E.B., Contact lens technology to 2020 and beyond: a review of recent patent literature. Clin Exp Optom, 2017. 100(5): p. 529-536.

141. Leahy, C.D. and LaBombard, D., Ocular drug delivery device. 2012, Vista Scientific LLC,: USA. p. 21.

142. Pimenta, A.F.R., Vieira, A.P., Colaco, R., Saramago, B., Gil, M.H., Coimbra, P., Alves, P., Bozukova, D., Correia, T.R., Correia, I.J., Guiomar, A.J., and Serro, A.P., Controlled release of moxifloxacin from intraocular lenses modified by Ar plasma-assisted grafting with AMPS or SBMA: An in vitro study. Colloids Surf B Biointerfaces, 2017. 156: p. 95-103.

143. Vieira, A.P., Pimenta, A.F.R., Silva, D., Gil, M.H., Alves, P., Coimbra, P., Mata, J., Bozukova, D., Correia, T.R., Correia, I.J., Serro, A.P., and Guiomar, A.J., Surface modification of an intraocular lens material by plasma-assisted grafting with 2-hydroxyethyl methacrylate (HEMA), for controlled release of moxifloxacin. Eur J Pharm Biopharm, 2017. 120: p. 52-62.

144. Bouledjouidja, A., Masmoudi, Y., Sergent, M., Trivedi, V., Meniai, A., and Badens, E., Drug loading of foldable commercial intraocular lenses using supercritical impregnation. International Journal of Pharmaceutics, 2016. 500(1): p. 85-99.

145. Mehta, P., Justo, L., Walsh, S., Arshad, M.S., Wilson, C.G., O'Sullivan, C.K., Moghimi, S.M., Vizirianakis, I.S., Avgoustakis, K., Fatouros, D.G., and Ahmad, Z., New platforms for multifunctional ocular lenses: engineering double-sided functionalized nano-coatings. J Drug Target, 2015. 23(4): p. 305-310.

146. Wertheimer, C., Kassumeh, S., Piravej, N.P., Nilmayer, O., Braun, C., Priglinger, C., Luft, N., Wolf, A., Mayer, W.J., Priglinger, S.G., and Eibl-Lindner, K.H., The Intraocular Lens as a Drug Delivery Device: In Vitro Screening of Pharmacologic Substances for the Prophylaxis of Posterior Capsule Opacification. Investigative Ophthalmology \& Visual Science, 2017. 58(14): p. 6408-6418.

147. Huang, X., Wang, Y., Cai, J.P., Ma, X.Y., Li, Y., Cheng, J.W., and Wei, R.L., Sustained release of 5-fluorouracil from chitosan nanoparticles surface modified intra ocular lens to prevent posterior capsule opacification: an in vitro and in vivo study. J Ocul Pharmacol Ther, 2013. 29(2): p. 208-215. 
148. Silva, G.C.M., Jabor, V.A.P., Bonato, P.S., Martinez, E.Z., and Faria, E.S.S.J., Penetration of $0.3 \%$ ciprofloxacin, $0.3 \%$ ofloxacin, and $0.5 \%$ moxifloxacin into the cornea and aqueous humor of enucleated human eyes. Braz J Med Biol Res, 2017. 50(7): p. e5901.

149. Freeman, P.D. and Kahook, M.Y., Preservatives in topical ophthalmic medications: historical and clinical perspectives. Expert Review of Ophthalmology, 2014. 4(1): p. 59-64.

150. Ahuja, M., Dhake, A.S., and Majumdar, D.K., Effect of formulation factors on in-vitro permeation of diclofenac from experimental and marketed aqueous eye drops through excised goat cornea. Yakugaku Zasshi, 2006. 126(12): p. 1369-1375.

151. Morrison, P.W. and Khutoryanskiy, V.V., Enhancement in corneal permeability of riboflavin using calcium sequestering compounds. Int J Pharm, 2014. 472(1-2): p. 56-64.

152. Rojanasakul, Y., Liaw, J., and Robinson, J.R., Mechanisms of Action of Some Penetration Enhancers in the Cornea - Laser Scanning Confocal Microscopic and Electrophysiology Studies. International Journal of Pharmaceutics, 1990. 66(1-3): p. 131-142.

153. Grass, G.M., Wood, R.W., and Robinson, J.R., Effects of calcium chelating agents on corneal permeability. Invest Ophthalmol Vis Sci, 1985. 26(1): p. 110-113.

154. Saettone, M.F., Chetoni, P., Cerbai, R., Mazzanti, G., and Braghiroli, L., Evaluation of ocular permeation enhancers: In vitro effects on corneal transport of four $\beta$-blockers, and in vitro/in vivo toxic activity. International Journal of Pharmaceutics, 1996. 142(1): p. 103-113.

155. Pescina, S., Carra, F., Padula, C., Santi, P., and Nicoli, S., Effect of $\mathrm{pH}$ and penetration enhancers on cysteamine stability and trans-corneal transport. Eur J Pharm Biopharm, 2016. 107(Supplement C): p. 171-179.

156. Madhu, C., Rix, P.J., Shackleton, M.J., Nguyen, T.G., and Tang-Liu, D.D., Effect of benzalkonium chloride/EDTA on the ocular bioavailability of ketorolac tromethamine following ocular instillation to normal and de-epithelialized corneas of rabbits. J Pharm Sci, 1996. 85(4): p. 415-418.

157. Kim, Y.C., Chiang, B., Wu, X., and Prausnitz, M.R., Ocular delivery of macromolecules. J Control Release, 2014. 190: p. 172-181.

158. Masson, M., Loftsson, T., Masson, G., and Stefansson, E., Cyclodextrins as permeation enhancers: some theoretical evaluations and in vitro testing. Journal of Controlled Release, 1999. 59(1): p. 107-118.

159. Bito, L.Z. and Baroody, R.A., The ocular pharmacokinetics of eicosanoids and their derivatives. 1. Comparison of ocular eicosanoid penetration and distribution following the topical application of PGF2 alpha, PGF2 alpha-1-methyl ester, and PGF2 alpha-1-isopropyl ester. Exp Eye Res, 1987. 44(2): p. 217-226.

160. Digiuni, M., Fogagnolo, P., and Rossetti, L., A review of the use of latanoprost for glaucoma since its launch. Expert Opin Pharmacother, 2012. 13(5): p. 723-745.

161. Daull, P., Buggage, R., Lambert, G., Faure, M.O., Serle, J., Wang, R.F., and Garrigue, J.S., A comparative study of a preservative-free latanoprost cationic emulsion (Catioprost) and a BAKpreserved latanoprost solution in animal models. J Ocul Pharmacol Ther, 2012. 28(5): p. 515523.

162. Sjoquist, B., Johansson, A., and Stjernschantz, J., Pharmacokinetics of latanoprost in the cynomolgus monkey. 3rd communication: tissue distribution after topical administration on the eye studied by whole body autoradiography. Glaucoma Research Laboratories. Arzneimittelforschung, 1999. 49(3): p. 240-249.

163. Kador, P.F., Blessing, K., Randazzo, J., Makita, J., and Wyman, M., Evaluation of the vascular targeting agent combretastatin a-4 prodrug on retinal neovascularization in the galactose-fed dog. J Ocul Pharmacol Ther, 2007. 23(2): p. 132-142.

164. Ibrahim, M.A., Do, D.V., Sepah, Y.J., Shah, S.M., Van Anden, E., Hafiz, G., Donahue, J.K., Rivers, R., Balkissoon, J., Handa, J.T., Campochiaro, P.A., and Nguyen, Q.D., Vascular disrupting agent for neovascular age related macular degeneration: a pilot study of the safety and efficacy of intravenous combretastatin A-4 phosphate. BMC Pharmacol Toxicol, 2013. 14: p. 7.

165. Cantor, L.B., Hoop, J., Wudunn, D., Yung, C.W., Catoira, Y., Valluri, S., Cortes, A., Acheampong, A., Woodward, D.F., and Wheeler, L.A., Levels of bimatoprost acid in the aqueous humour after bimatoprost treatment of patients with cataract. Br J Ophthalmol, 2007. 91(5): p. 629-632.

166. Wertheimer, C., Kassumeh, S., Piravej, N.P., Nilmayer, O., Braun, C., Priglinger, C., Luft, N., Wolf, A., Mayer, W.J., Priglinger, S.G., and Eibl-Lindner, K.H., The Intraocular Lens as a Drug Delivery Device: In Vitro Screening of Pharmacologic Substances for the Prophylaxis of Posterior Capsule Opacification. Invest Ophthalmol Vis Sci, 2017. 58(14): p. 6408-6418. 
167. Bouledjouidja, A., Masmoudi, Y., Sergent, M., Trivedi, V., Meniai, A., and Badens, E., Drug loading of foldable commercial intraocular lenses using supercritical impregnation. Int J Pharm, 2016. 500(1-2): p. 85-99.

168. Katz, I.M. and Blackman, W.M., A soluble sustained-release ophthalmic delivery unit. Am J Ophthalmol, 1977. 83(5): p. 728-734.

169. Papas, E.B., The significance of oxygen during contact lens wear. Cont Lens Anterior Eye, 2014. 37(6): p. 394-404.

170. Garcia-Millan, E., Quintans-Carballo, M., and Otero-Espinar, F.J., Improved release of triamcinolone acetonide from medicated soft contact lenses loaded with drug nanosuspensions. Int J Pharm, 2017. 525(1): p. 226-236.

171. Hu, X., Tan, H., Chen, P., Wang, X., and Pang, J., Polymer Micelles Laden Hydrogel Contact Lenses for Ophthalmic Drug Delivery. J Nanosci Nanotechnol, 2016. 16(6): p. 5480-5488.

172. Paradiso, P., Colaco, R., Mata, J.L.G., Krastev, R., Saramago, B., and Serro, A.P., Drug release from liposome coated hydrogels for soft contact lenses: the blinking and temperature effect. J Biomed Mater Res B Appl Biomater, 2017. 105(7): p. 1799-1807.

173. Rhee, M.K. and Mah, F.S., Cataract Drug Delivery Systems (Dropless vs. Nondropless Cataract Surgery). Int Ophthalmol Clin, 2016. 56(3): p. 117-136.

174. Eydelman, M.B., Nguyen, T., and Green, J.A., The US Food and Drug Administration's New Regulatory Toolkit to Bring Medical Device Innovation Back to the United States. JAMA Ophthalmol, 2016. 134(4): p. 353-354. 


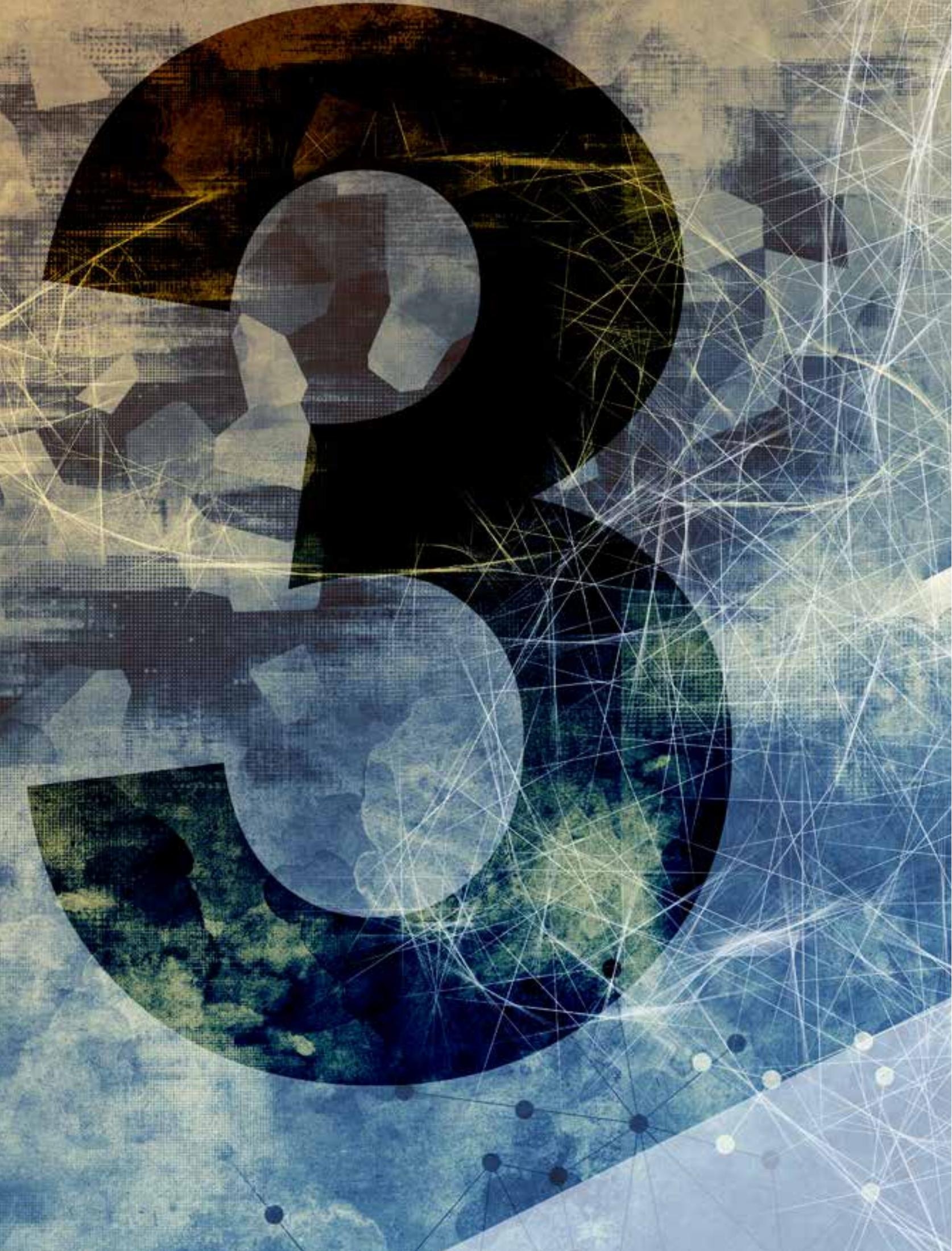


chapier 3.

\section{Design of the ocular coiln, a new} @evice for nonninvasive drug

delivery

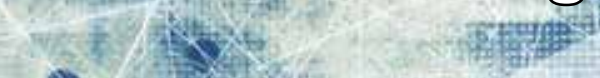




\section{Abstract}

Eye drops and ointments are the most prescribed methods for ocular drug delivery. However, due to low drug bioavailability, rapid drug elimination, and low patient compliance there is a need for improved ophthalmic drug delivery systems. This study provides insights into the design of a new drug delivery device that consists of an ocular coil filled with ketorolac loaded PMMA microspheres.

Nine different ocular coils were created, ranging in wire diameter and coiled outer diameter. Based on its microsphere holding capacity and flexibility, one type of ocular coil was selected and used for further experiments. No escape of microspheres was observed after bending the ocular coil at curvature which reflect the in vivo situation in human upon positioning in the lower conjunctival sac.

Shape behavior and tissue contact were investigated by computed tomography imaging after inserting the ocular coil in the lower conjunctival fornix of a human cadaver. Thanks to its high flexibility, the ocular coil bends along the circumference of the eye. Because of its location deep in the fornix, it appears unlikely that in vivo, the ocular coil will interfere with eye movements.

In vitro drug release experiments demonstrate the potential of the ocular coil as sustained drug delivery device for the eye. We developed PMMA microspheres with a $26.5 \pm 0.3 \mathrm{wt} \%$ ketorolac encapsulation efficiency. After 28 days, $69.9 \% \pm 5.6 \%$ of the loaded ketorolac was released from the ocular coil when tested in an in vitro lacrimal system. In the first three days high released dose $(48.7 \% \pm 5.4 \%)$ was observed, followed by a more gradually release of ketorolac. Hence, the ocular coil seems a promising carrier for ophthalmic drugs delivery in the early postoperative time period.

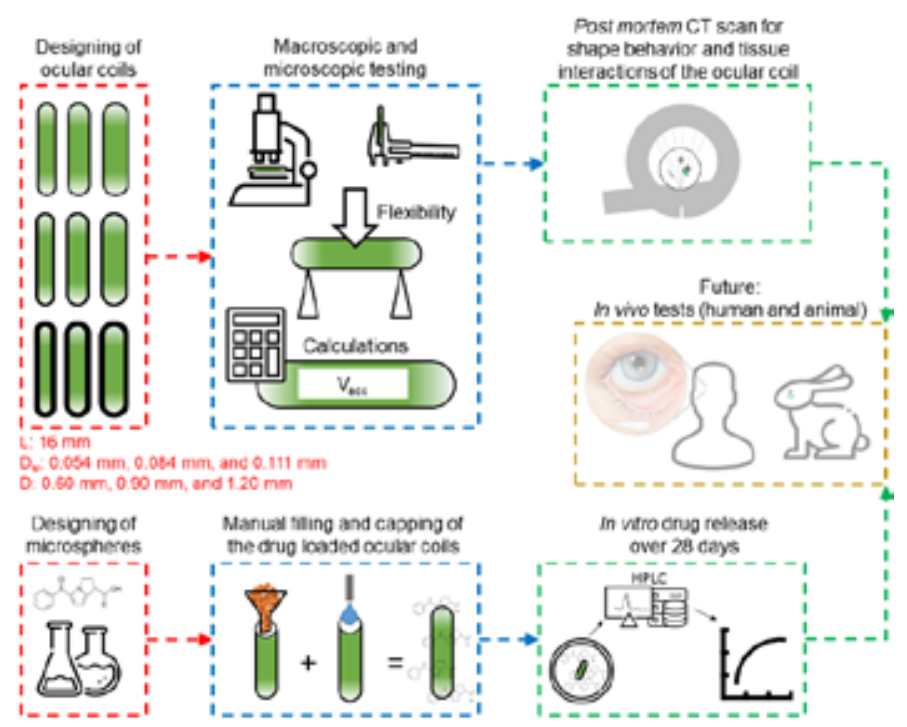

Graphical abstract 


\subsection{Introduction}

The world market for ophthalmological products is valued at approximately USD 50 billion, of which pharmaceuticals take up 44\%.[1] Most pharmaceutical agents (drugs) are administered via eye drops or ointments. However, these drug delivery methods have several drawbacks such as low drug bioavailability[2-5], rapid drug elimination[6,7], side effects (such as allergies)[8] and low patient compliance.[9-13] Therefore, new drug delivery methods have been developed, such as intravitreal injectable inserts (e.g. Ozurdex ${ }^{\circledR}$ (Allergan, Dublin, IE) or lluvien ${ }^{\circledR}$ (Alimera Sciences, Alpharetta, GA, US)) and conjunctival inserts (e.g. Mydriasert ${ }^{\circledR}$ (Thea Pharmaceutical, Clermont-Ferrand, FR), Lacrisert ${ }^{\circledR}$ (Bausch \& Lomb, Rochester, NY US), Ocusert ${ }^{\circledR}$ (ALZA, Mountain View, CA, US))[14]. Several clinical trials with new drug delivery devices including the Helios ring ${ }^{\mathrm{TM}}$ (Allergan, Dublin, IE), a bimatoprost eluting ring for glaucoma treatment $[15,16]$ and Dextenza ${ }^{\circledR}$ (Ocular Therapeutix, Bedford, MA, US), a dexamethasone releasing punctum plug $[17,18]$ have been conducted. The bimatoprost ring had a retention percentage above $90 \%$ in its phase 2 study for 6 and 13 months, but could not lower the intraocular pressure equally to a timolol $0.5 \%$ ophthalmic solution. $[15,16]$ The Dextenza ${ }^{\circledR}$ punctum plug was efficacious compared to an empty vehicle in a phase 2 trial for the treatment of allergic conjunctivitis.[18] In a second study, Dextenza ${ }^{\circledR}$ demonstrated clinically significant reductions in anterior chamber cells, flare and pain after cataract surgery.[17] These studies show that treatment with sustained drug delivery devices can reach similar efficacies without the daily burden of applying drugs.

In 2004, we reported our results on an ocular coil for drug delivery [19-22] that consists of a coiled coated stainless steel wire, filled with a drug eluting matrix in its inner lumen. The ocular coil is closed on both extremities with a dome-shaped UVcurable acrylate urethane cap to soften its extremities while maintaining the drug eluting matrix inside (figure 3-1). Filling the ocular coil with drug-coated wire filaments resulted in an ocular coil which was too rigid in vivo to stay comfortably in the lower fornix of the eye. However, no adverse events have occurred. As a subsequent improvement, we developed microsphere-filled ocular coils, which were shown to have improved flexibility as measured by a triple point bending test.[19]

The aim of this study is to provide insights into the physical characteristics of the microsphere-filled ocular coil. We investigated the effect of several different diameters of stainless steel wire on the flexibility of the ocular coil under the assumption that higher flexibility might correlate to higher comfort for the patient. In addition, we studied the effect of variable outer diameters of the ocular coil on its flexibility. Furthermore, the effect on flexibility after filling the inner lumen with polymethyl methacrylate (PMMA) microspheres was tested. PMMA was used since it has proven not to degrade or decompose in the human body; besides, it does not 
provoke an immune response when used for ocular purposes.[23] However, in order to ensure that the microspheres remain inside the ocular coil, we calculated the gapspace between the turn-windings to prevent escape. We measured in vitro escape of microspheres from the ocular coil. Based on the these experiments, one type of ocular coil was selected and was inserted in the lower conjunctival fornix of a formalin fixed human cadaver in order to visualize the interaction between the anatomical boundaries of the eye and shape behavior of the ocular coil for further clinical applications. Finally, with potential application in a post-operative setting, we investigated the use of ketorolac, a non-steroidal anti-inflammatory drug (NSAID) for drug release. Ketorolac tromethamine is used for the prevention of Cystoid Macular Edema, a common complication of cataract surgery. The in vitro drug release profile of the ocular coil filled with ketorolac-loaded microspheres was tested and compared to the release profile of eye drops.

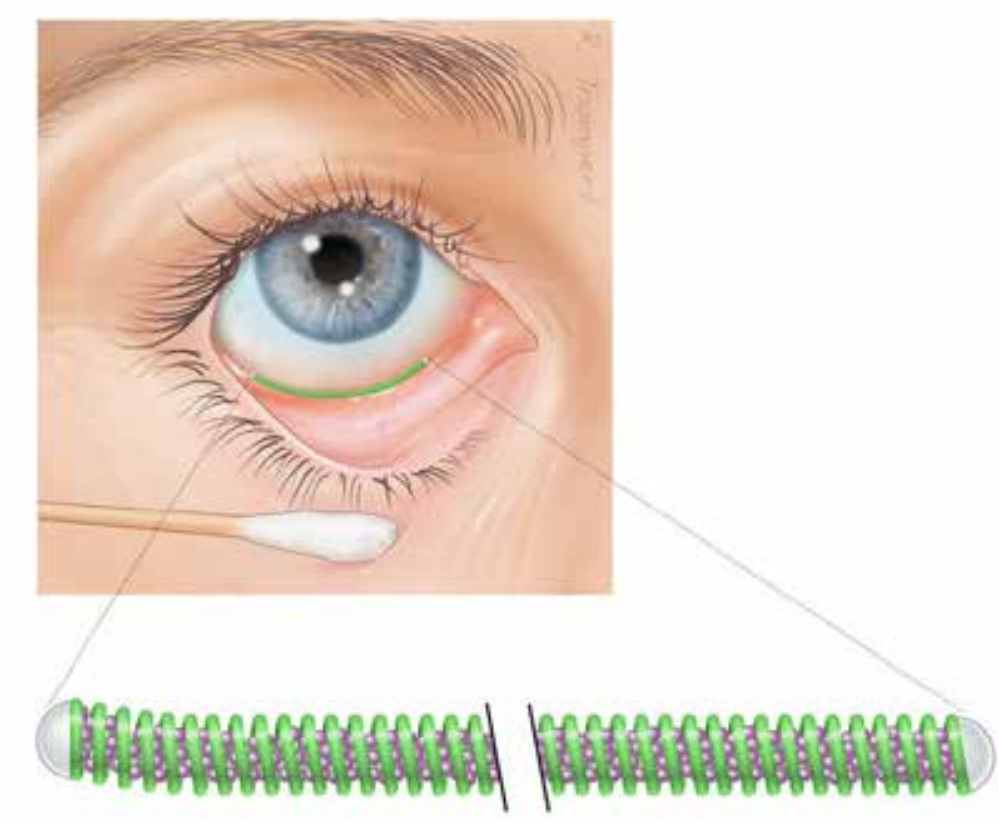

Figure 3-1. An ocular coil positioned in the conjunctival fornix of the lower eyelid. Insert shows an ocular coil which is filled with a microsphere matrix and capped on both sides with UV-curable acrylate urethane glue. Drawn by R. Trompert. 


\subsection{Materials and methods}

\subsubsection{Materials}

Different sizes of ocular coils were ordered from EPflex (Dettingen an der Erms, DE). A lens folding forceps for handling of the ocular coil was bought at Malosa medical (\#1131, Malosa Medical, Elland, UK). Polymethylmethacrylate (PMMA, Mn $\approx 43$ $\mathrm{kg} / \mathrm{mol}$, Diakon MG102) and PMMA microspheres of $155 \mu \mathrm{m} \pm 15 \mu \mathrm{m}$ in diameter (\#009011-14-7) were purchased from Lucite International (Cumberland Place, UK). Polyvinyl alcohol (PVOH, $\mathrm{Mn} \approx 80 \mathrm{~kg} / \mathrm{mol}$, Mowiol 8-88), potassium dihydrogen phosphate $\left(\mathrm{KH}_{2} \mathrm{PO}_{4}\right)$, and dichloromethane $\left(\mathrm{CH}_{2} \mathrm{Cl}_{2}\right)$ were used as received, and were bought at Sigma-Aldrich (Saint Louis, Missouri, USA). The water used for the synthesis of the particles was filtered on a Millipore Milli-Q plus system $(R=18.2$ $\mathrm{m} \Omega$ ). Toluene (99.8\% HiPerSolv) and micro-sieves (450, 160, 140 and $70 \mu \mathrm{m})$ were purchased from VWR (VWR international, Oud-Heverlee, BE). Ketorolac tromethamine was purchased from MSN laboratories (Telangana, IN)

\subsubsection{Ocular coil size and shape}

Ocular coils, made of coated stainless steel (SS304) with a total length (L) of $16 \mathrm{~mm}$, and an outer diameter ( $\left.D_{0}\right)$ of $0.6 \mathrm{~mm}, 0.9 \mathrm{~mm}$ or $1.2 \mathrm{~mm}$ and a wire diameter ( $D_{\mathrm{w}}$ ) of $0.054 \mathrm{~mm}, 0.084 \mathrm{~mm}$ or $0.111 \mathrm{~mm}$ were purchased from EPflex with tolerances as mentioned in table 3-1 and figure 3-2.

The ocular coils were filled using a proprietary funnel-volume based technology that allowed filling of the lumen of ocular coils with PMMA microspheres. Filling of 103 ocular coils with $3.0 \mathrm{mg}$ PMMA microspheres was analyzed and gave a Gaussion curve according to the D'Agostino's $K^{2}$ test.

The dimensions of the ocular coils were manually measured with a caliper (\#1150MI, IHM, Seynod, FR). Subsequent, scanning electron microscopic (SEM) pictures were taken with a JSM 6010 Plus/LV (JEOL, Tokyo, JP).

\subsubsection{Flexibility tests}

Flexibility tests were performed to determine whether filling the inner lumen of the ocular coil with microspheres affects their flexibility. The flexibility of the ocular coils was measured through a three-point bending test [19] using a Rheometric solids analyser (RSA3, TA instruments, Lukens Drive New Castle, DE, US), equipped with RSI-Orchestrator software (version 6.5) (TA instruments, Lukens Drive New Castle, DE, US). The ends of each ocular coil were placed onto two solid points and a force was applied to the center of the ocular coil. The displacement was set at $2.5 \mathrm{~mm}$ and the force as a function of the displacement was measured at the center of the ocular coil (figure 3-5a). To compare flexibility between the different ocular coils, empty and microsphere filled ocular coils were compared using unpaired t-test. 


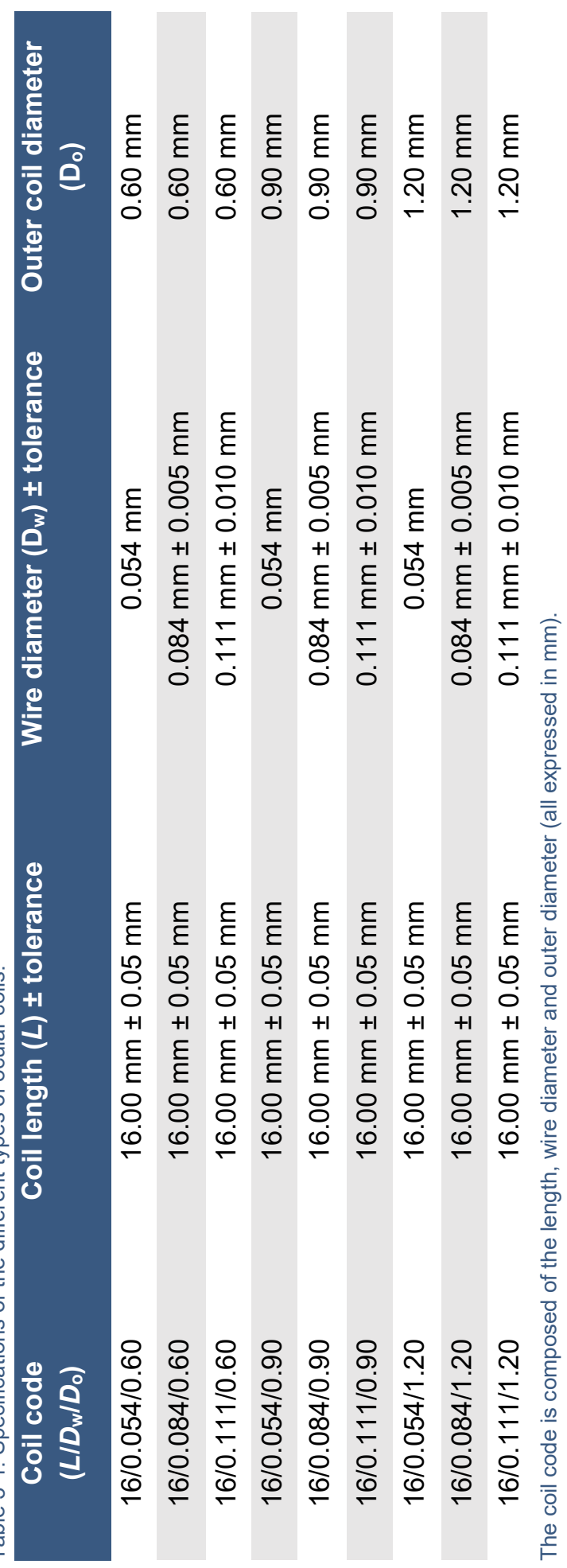



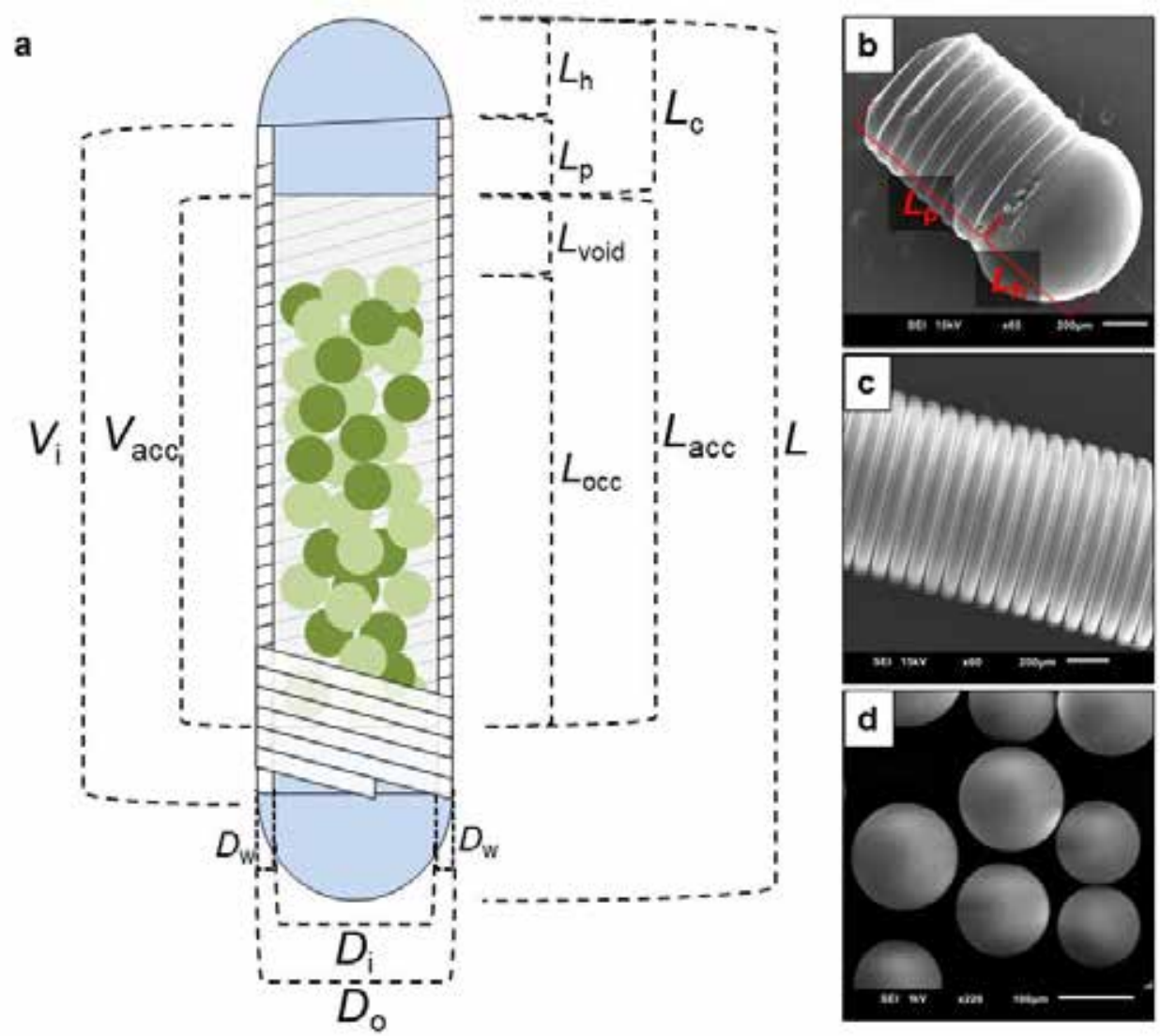

Figure 3-2. (a). Technical drawing of the ocular coil. $L$ is the total length of the ocular coil consisting of: Length of the cap $\left(L_{c}\right)$ which consists of cap head length $\left(L_{h}\right)$ and cap plug length $\left(L_{p}\right)$. The accessible length $\left(L_{\mathrm{acc}}\right)$ consists out of void length $\left(L_{\text {void }}\right)$ and occupied length $\left(L_{\mathrm{occ}}\right)$. The outer diameter $\left(D_{\circ}\right)$ of the ocular coil is based on the wire diameter $\left(D_{\mathrm{w}}\right)$ and the inner diameter $\left(D_{\mathrm{i}}\right)$. The ocular coil has an inner volume $\left(V_{\mathrm{i}}\right)$ which is partly accessible $\left(V_{\text {acc }}\right)$. (b) Shows scanning electron microscopy (SEM) photo of the cap, including a cap head $\left(L_{h}\right)$ and the cap plug $\left(L_{p}\right)$ (magnification x65). (c) SEM photo from the central part of the ocular coil (magnification x60) and (d) SEM photo from the PMMA microspheres (magnification x220). SEI = secondary electron imaging.

\subsubsection{Gap size between the turn-windings}

Analysis of the gap size between the turn-windings was performed in order to assure that microspheres do not escape from the ocular coil. Ocular coils were bent on curved glass discs with a diameter of $24 \mathrm{~mm}$ (average diameter of the ocular globe[24]). Bend and stretch forces were measured on the ocular coil using proprietary equipment. Images of bent coils were taken using an optical microscope (BX51, Olympus, JP) equipped with $4 \mathrm{X}$ and $10 \mathrm{X}$ objectives and a digital camera 
(SC50, Olympus, JP). The images were analyzed using the ruler tool in Adobe Photoshop CC 2015 (Adobe Systems Inc., San Jose, CA, US).

The measured gap sizes between the turn-windings were compared to a Gaussian curve, with good fit, using the D'Agostino's $\mathrm{K}^{2}$ test. The mean values of the measured gap size between the turn-windings was compared to the calculated mean gap size between the turn-windings when bent along the circumference of a disk with a diameter of $24 \mathrm{~mm}$, using the student t-test. Furthermore, the measured mean gap size between the turn-windings was also compared to the calculated mean maximum gap size between turn-windings (see section 3.3.1 Estimation of the gap size between the turn-windings of the ocular coil) using an unpaired student t-test.

\subsubsection{Post mortem ocular coil insertion and computed tomography imaging}

For this study the head of an intact human cadaver specimen from the Maastricht University body donation program was used. A handwritten and signed codicil from the donor is kept at the Department of Anatomy and Embryology, Faculty of Health, Medicine and Life Sciences, Maastricht University, Maastricht, the Netherlands. Comfort of the ocular coil in vivo is related to the outer surface of the ocular coil, flexibility of the ocular coil and its capacity to follow the anatomical boundaries of the adnexa of the orbita (e.g. muscles and other soft tissues). To gain more insight in shape behavior of the ocular coil, the ocular coil was inserted in the lower conjunctival fornix of the head of a formalin fixed human cadaver. The ocular coil (16/0.084/0.90, see table 3-1) was inserted in the fornix using a lens folding forceps. A computed tomography (CT) scan was made with a multi-detector helical scanner (SOMATOM force, Siemens Healthcare, Forchheim, DE). The head was scanned in the supine position using the following technical parameters: $192 \times 0.6 \mathrm{~mm}$ collimation, $100-120 \mathrm{kV}, 50-461 \mathrm{~mA}, 0.5-1 \mathrm{~s}$ scan time, and $0.6 \mathrm{~mm}$ section thickness. The scan was analyzed with Versalius 3D $\vee 1.0$ (ps-medtech B.V., Amsterdam, NL) and Photoshop CC 2015 (Adobe Systems inc., San Jose, CA, US).

\subsubsection{Oil/water emulsification for encapsulation of ketorolac tromethamine into PMMA microspheres}

Ketorolac tromethamine loaded PMMA microspheres were prepared according to an oil/water emulsification method, modified from Govender et al.[25] The organic phase was prepared by adding $5 \mathrm{~mL}$ (corresponding to $746 \mathrm{mg}$ PMMA) of a $10 \%$

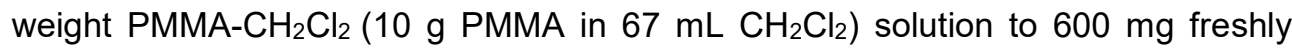
grounded ketorolac tromethamine. The suspension was homogenized by gentle shaking and ultrasonic bath treatment for $15 \mathrm{~min}$. The aqueous phase consisted of a $100 \mathrm{~mL}$ PVOH solution (1\% weight) in MilliQ water supplemented with 6 gram of 
$\mathrm{KH}_{2} \mathrm{PO}_{4}$. The organic phase was then poured in one shot to the aqueous phase at room temperature and stirred for 1 hour at 500 RPM using a mechanical stirrer (Heidolph RZR 2021, Heidolph instruments, Schwabach, DE). After stirring, the microspheres were washed three times with $100 \mathrm{~mL}$ MilliQ water and recovered on filter paper. In order to collect microspheres of $150 \pm 10 \mu \mathrm{m}$ diameter, the microspheres were transferred onto a stack of sieves (from 450, 160, 140 to $70 \mu \mathrm{m}$ ) under a continuous tap water flow for $10 \mathrm{~min}$. After sieving, the microspheres were collected by washing off the $140 \mu \mathrm{m}$ sieve with water, filtrated on a paper filter and freeze-dried before being stored at $23^{\circ} \mathrm{C}$ in the absence of light. To determine the encapsulation efficacy, $4 \mathrm{mg}$ of microspheres was dissolved into $10 \mathrm{~mL}$ toluene. Protocol was followed as described in section 2.8. A total of $26.5 \pm 0.3 \mathrm{wt} \%$ ketorolac was recovered from $150 \pm 10 \mu \mathrm{m}$ sized microspheres indicating the encapsulation efficacy.

The use of latter materials resulted in approximately $200 \mathrm{mg}$ dry microspheres with a size of $150 \pm 10 \mu \mathrm{m}$. The production yield of $19.7 \%$ was calculated based on the starting amount of PMMA (746 mg) and the resulting amount of PMMA in the dry microspheres (200 $\mathrm{mg}$ dry microspheres $-26.5 \mathrm{wt} \%$ ketorolac $=147 \mathrm{mg}$ PMMA).

\subsubsection{In vitro drug release}

Simulated tear fluid (STF) was prepared according to the description of Zhang et al. [26] and was used with a pH of 7.4. Ocular coils were submerged in $2.5 \mathrm{~mL}$ STF in one well of a 12-well-plate. In order to simulate physiological tear flow conditions, we designed an in vitro lacrimal system. One syringe pump created a continuous inflow of $2 \mu \mathrm{L} / \mathrm{min}$ STF whereas another syringe pump provided a continuous outflow of 2 $\mu \mathrm{L} /$ min STF (figure 3-S1). The experiment was performed in the dark at room temperature for 28 days. Parafilm and a silicon inlay were used to prevent evaporation. At specific time intervals during the first day (10 min, $30 \mathrm{~min}, 1$ hour, 4 hours, 8 hours, 24 hours, 32 hours, and 48 hours), samples of $60 \mu \mathrm{L}$ were drawn from the solution in the well. During the following days (day $3,6,7,9,10,13,15,17$, $20,22,24,27$, and 28 ), samples were drawn by collection the solution from the outflow syringes.

A similar experimental set-up was used to measure drug release from ketorolac eye drops $\left(0,5 \%\right.$ Acular $^{\mathrm{TM}}$, Allergan, Dublin, IE). A drop $(50 \mu \mathrm{L})$ of ketorolac ophthalmic solution was added to the well at $0,4,8,24,28$ and 32 hours. Samples were taken right before and after addition of the eye drop. The concentration of ketorolac was measured using a Nanodrop 1000 spectrophotometer (Thermo Scientific, Waltham, MA, USA) at $313 \mathrm{~nm}$. All data are expressed as mean \pm SD. 


\subsubsection{Liquid-liquid extraction of ketorolac-loaded microspheres}

To determine the remaining ketorolac content in the microspheres after the in vitro drug release experiment, the microspheres within the ocular coils were dissolved in $10 \mathrm{~mL}$ toluene, and ketorolac was extracted from the liquid using water (liquid-liquid extraction). One ocular coil was placed in a beaker with $10 \mathrm{~mL}$ toluene, the windings were gently pulled open using tweezers, and stirred overnight (at least 12 hours) using a magnetic stirrer. Afterwards, two fractions of MilliQ water (10 mL and $40 \mathrm{~mL}$ ) were added while stirring and the solution was stirred for 2 hours per fraction. Finally, the aqueous phase was collected by decantation and measured at $313 \mathrm{~nm}$ with a Nanodrop 1000 spectrophotometer (Thermo Scientific, Waltham, MA, USA).

\subsection{Calculations}

\subsubsection{Estimation of the gap size between the turn-windings of the ocular coil}

To estimate the optimal size of microspheres, which prevent an escape from the ocular coil, the gap size between the turn-windings was predicted. The ocular coil is made of a coiled wire characterized by a total length $(L)$, wire diameter $\left(D_{\mathrm{w}}\right)$, and outer diameter $\left(D_{\circ}\right)$ (figure 3-3a). When the ocular coil is bent (not considering stretching or other torsions) we assume that the maximum gap size between the turn-windings is realized if both extremities of the ocular coil would touch each other $\left(\mathrm{A}=\mathrm{A}^{\prime}\right.$ and $\left.\mathrm{B}=\mathrm{B}^{\prime}\right)$, implies an applied rotation of $\alpha=360^{\circ}$, as shown in figure 3-3b. In such a case, $r_{\mathrm{i}}$ and $r_{\mathrm{o}}$ (figure 3-3b) represent the inner and outer radius of the bent coil respectively, whereas the internal and external circumferences are indicated with $c_{\mathrm{i}}$ and $c_{\mathrm{o}}$. For $\alpha=360^{\circ}$, the inner circumference $\left(c_{\mathrm{i}}\right)$ equals $\mathrm{L}$ (equation 1. $c_{\mathrm{i}}=L=$ $2 \pi r_{\mathrm{i}}$ ) whereas, because the stainless steel wire cannot be compressed, the outer circumference $\left(c_{0}\right)$ equals (equation 2 ). The circumference follows from (equation 3 ). The quantity $\Delta L$ is calculated from combining Equations 1, 2, and 3: (equation 4).

$$
\begin{aligned}
& c_{\mathrm{o}}=c_{\mathrm{i}}+\Delta L \\
& c_{\mathrm{o}}=2 \pi r_{\mathrm{o}}=2 \pi\left(r_{\mathrm{i}}+D_{\mathrm{o}}-\frac{D_{\mathrm{w}}}{2}\right) \\
& \Delta L=2 \pi\left(r_{\mathrm{i}}+D_{\mathrm{o}}-\frac{D_{\mathrm{w}}}{2}\right)-2 \pi\left(r_{\mathrm{i}}\right)=2 \pi\left(D_{\mathrm{o}}-\frac{D_{\mathrm{w}}}{2}\right)
\end{aligned}
$$

The gap size between the turn-windings of the ocular coil (defined as: $\lambda$ ) is obtained from $\lambda=\frac{\Delta L}{N}$. Combining this with the calculation for the number of windings, $N=\frac{L}{D_{\mathrm{w}}}$, the gap size for each ocular coil can be calculated from $L, D_{\circ}$ and $D_{\mathrm{w}}$ (equation 5). Interestingly, equation 6 can be generalized and the gap size between the turnwindings can be predicted for any given rotation $\alpha\left(\lambda=\alpha \frac{D_{0}}{N}\right)$. 


$$
\lambda=2 \pi \frac{\left(D_{\mathrm{o}}-\frac{D_{\mathrm{w}}}{2}\right) D_{\mathrm{w}}}{L}
$$

However, in clinical practice it is more convenient to use the diameter of the eye to estimate the bending of the ocular coil. Therefore, we translated the equations to a clinical situation. When the eye is observed from a frontal plane, the globe can be represented by a circle (figure 3-3c). Since the location of the ocular coil is expected to be at the lower side of the eye, according to the curve of the globe, the outer circumference of the eye $\left(c_{\text {o(eye) }}\right)$ equals the length of the ocular coil $(L)$ plus a variable $(x)$. Since the gap size between the turn-windings is dependent on the outer circumference of the eye ( $\left.c_{\circ(\text { eye })}\right)$, the $D_{\circ}$ and the $D_{\mathrm{w}}$, these variables can be used to calculate the gap size between the turn-windings: (equation 6 ). Thereby assuming the length of the ocular coil equals the length of $c_{o(\text { eye). }}$

$$
\lambda=2 \pi \frac{\left(D_{\mathrm{o}}-\frac{D_{\mathrm{w}}}{2}\right) D_{\mathrm{w}}}{c_{\mathrm{o}(\text { eye })}}
$$

\subsubsection{Calculation of microsphere mass of the inner lumen of the ocular coil}

To estimate the available volume of the ocular coil, the inner volume of the ocular coil was calculated. The inner lumen, i.e. the central cavity, of the ocular coil has a cylindrical shape $\left(V=\pi r^{2} L\right)$. When calculating the accessible inner volume of the ocular coil $\left(V_{\text {acc }}\right)$, the total accessible length $\left(L_{\text {acc }}\right)$ equals the length $(L)$ of the ocular coil subtracted with the length of the two cap heads $\left(L_{h}\right)$ and cap plugs $\left(L_{p}\right)\left(L_{h}+L_{p}\right.$ is the total length of the cap $\left(L_{c}\right)$ ) create (figure 3-2). Besides the caps, also the diameter of the wire $\left(D_{\mathrm{w}}\right)$ is subtracted twice from the outer diameter $\left(D_{0}\right)$ to estimate the inner diameter $\left(D_{\mathrm{i}}\right)$ (see figure 3-2).

We estimate that the maximum concentration of microspheres correspond to the random close packing (rcp) volume fraction. The rcp volume fraction $\eta$ of identical (monodisperse) spheres results in a packing density of 0.64.[27-29] Therefore, we used $\eta=0.64$ as packing density inside $V_{\text {acc }}$ of the ocular coil.

The inner volume ( $\left.V_{\text {acc }}\right)$ can be split in two compartments: the occupied volume ( $V_{\text {occ }}$ ) which represents the volume occupied by the microspheres and the empty or free volume $\left(V_{\text {void }}\right)$. The ratio of $V_{\text {occ }}$ over $V_{\text {acc }}$ is expressed as $\%$ filling. The total mass of the microspheres ( $m_{\text {spheres}}$ ) inside the ocular coil is computed from (equation 7$)$. The density $(\rho)$ of PMMA is: $1.18 \mathrm{~g} / \mathrm{cm}^{3}$ [MSDS Poly(methyl methacrylate) \#182230, Sigma-Aldrich].

$$
m_{\text {spheres }}=\left(\%_{\text {filling }} \times V_{\text {acc }}\right) \times \rho \times \eta=V_{\text {occ }} \times \rho \times \eta
$$


a

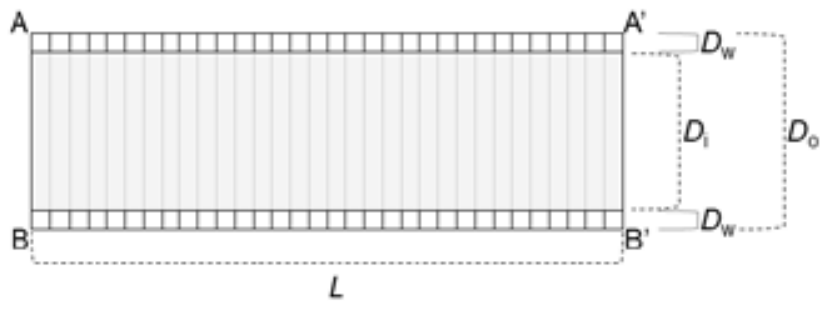

b

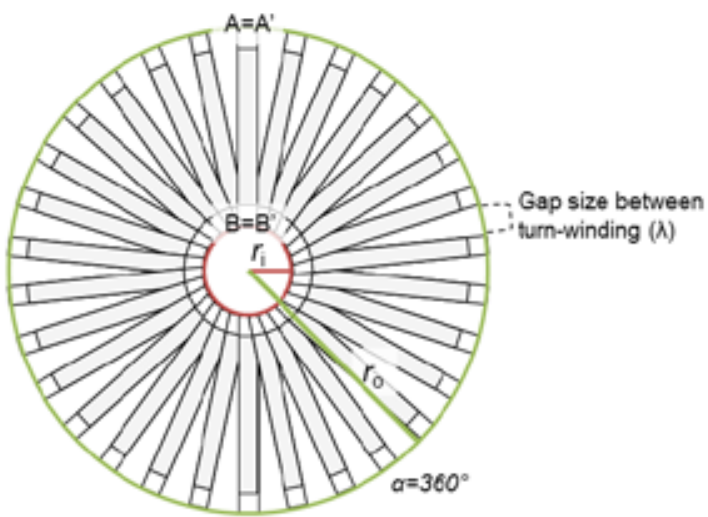

c

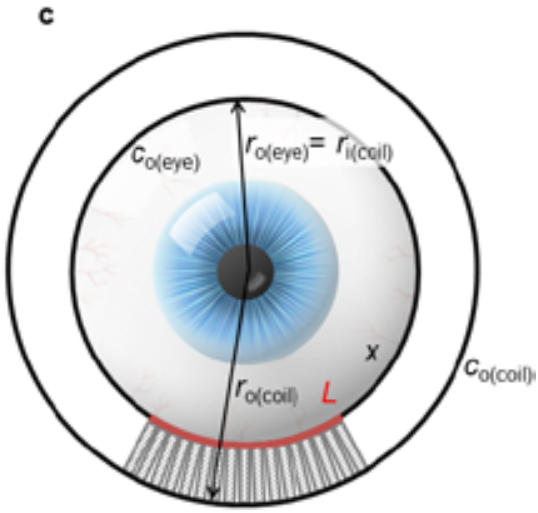

Figure 3-3. Schematic drawing of the bending of an ocular coil. (a) Overview of the ocular coil variables, the extremities are named as A with $A^{\prime}$ on the contralateral side and B with B'. The ocular coil is characterized by its length $(L)$, an outer diameter $\left(D_{0}\right)$ and wire diameter $\left(D_{\mathrm{w}}\right)$. (b) Bending the ocular coil to its own extremities results in a circle with a rotation $\alpha$ of $360^{\circ}$. (c) Theoretical location of the ocular coil in the conjunctival fornix, where the ocular coil bends along the curvature of the eye. The length of the ocular coil is represented with the red curve $(L)$ on the outer circumference of the eye $\left(c_{\text {o(eye) }}\right)$ and bending of the coil is in line with the outer circumference of the coil $\left(c_{\text {o(coil) }}\right)$. The outer radius of the eye $\left(r_{\text {o(eye) }}\right)$ and the outer radius of the ocular coil $\left(r_{\text {o(coili }}\right)$ are depicted by arrows. The inner and outer circumference $\left(c_{\mathrm{i}}\right.$ and $c_{\circ}$ ) are indicated.

\subsubsection{Process variation by a Monte Carlo simulation}

To include a simulated process variation, a Monte Carlo simulation was used with 2000 samples. Table 3-2 gives an overview of the average values, set tolerances (if applicable) and standard deviations which were used.

Table 3-2. Values for the Monte Carlo simulation

\begin{tabular}{llll} 
& Average & Tolerance & Stdev \\
\hline$L(\mathrm{~mm})$ & 16.0 & 0.5 & 0.17 \\
$L_{\mathrm{c}}(\mathrm{mm})$ & 0.76 & N.A. & 0.10 \\
Microspheres $(\mathrm{mg})$ & 3.00 & 0.30 & 0.10 \\
$D_{\circ}(\mathrm{mm})$ & 0.90 & 0.02 & 0.007 \\
$D_{\mathrm{w}}(\mathrm{mm})$ & 0.084 & 0.005 & 0.002
\end{tabular}




\subsection{Results}

\subsubsection{Ocular coils}

To design an optimal ocular coil for drug delivery purposes, ocular coils with a total length $L=16.0 \mathrm{~mm} \pm 0.5 \mathrm{~mm}$ (including caps) and different wire thicknesses and outer diameters were used to calculate the inner volume of each type of ocular coil. The measured inner volume was obtained by subtracting the average cap lengths (provided by the manufacturer) from the measured lengths of the ocular coil. The calculated inner volume was computed using experimentally derived and Monte Carlo simulated values for the $L_{,} L_{c}, L_{\mathrm{n}}$ and $L_{\mathrm{p}}$ of ten ocular coils. When the measured values were compared to the calculated values, small differences in the accessible volume were observed for ocular coils 16/0.111/1.20, 16/0.084/1.20, and 16/0.111/0.90 (figure 3-4a). Ocular coil 16/0.054/1.20 could not be produced, due to the large $D_{o}$ and the thin $D_{w}$ the ocular coil was unstable and could not hold its shape. Because of the Monte Carlo simulation no tests were executed to test significance of the differences. Due to large samples in the calculated groups all differences would show significance.

Based on these inner volume calculations, the filling volume of the ocular coil was estimated by considering a packing density of 0.64 and $70 \%$ filling with monodisperse PMMA microspheres. From figure $3-4 b$ it follows that filling volume increases with increasing outer diameter and decreasing wire diameter.
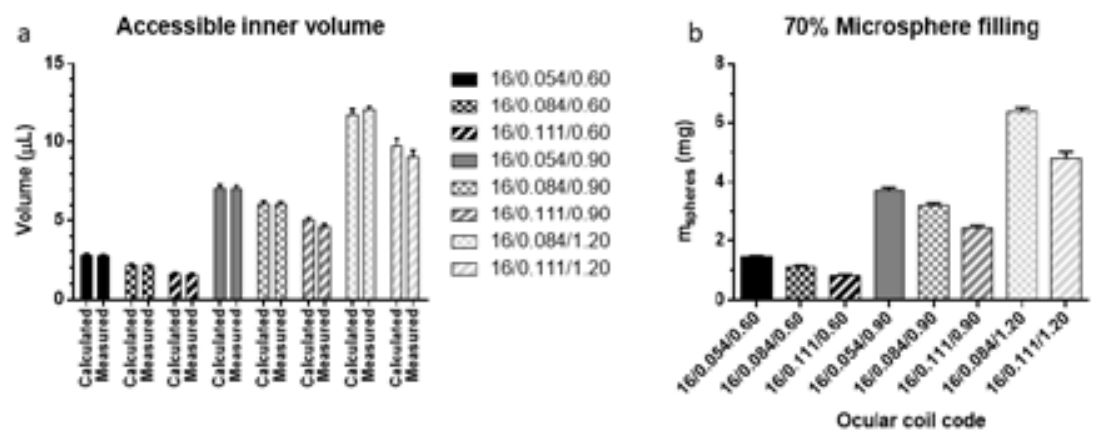

Figure 4. (a) Accessible inner volume of the ocular coils: calculated and measured. (b) Calculated weight of microspheres to be filled in the ocular coil assuming $70 \%$ filling volume and a packing density of 0.64 $\mathrm{g} / \mathrm{cm}^{3}$ based on the measured volumes of the ocular coils and PMMA density of $1.18 \mathrm{~g} / \mathrm{cm}^{3}$.

\subsubsection{Flexibility of ocular coils}

Flexibility of the ocular coil is an important factor that can affect comfort. Previous results indicate that filling the ocular coil with drug-loaded wire-filaments affect the ocular coil's flexibility.[19] Therefore, the ocular coils in this study were filled with microspheres. Flexibility of the ocular coils (empty and filled) was measured with a 
three point bending test, see figure 3-5a. The required forces measured to bend the ocular coils with wire diameter of $0.054 \mathrm{~mm}$ were below the detection limit $(0.0005$ $\mathrm{N} / \mathrm{mm}$ ). The ocular coils with an outer-diameter of $0.60 \mathrm{~mm}, 0.90 \mathrm{~mm}$, and $1.20 \mathrm{~mm}$, and a wire thickness of $0.084 \mathrm{~mm}$, and ocular coils with an outer diameter of 0.60 $\mathrm{mm}$, and $0.90 \mathrm{~mm}$ with a wire thickness of $0.111 \mathrm{~mm}$ do not show difference in stiffness when filled with microspheres during displacement in the three point bending test. Only for ocular coil 16/0.111/1.20 a significant difference $(p<0.05)$ in stiffness between the filled and empty condition was detected, as shown in figure 3$5 b$.

\subsubsection{Filling of the ocular coils with PMMA microspheres}

Based on the results of the filling (table 3-3) and flexibility (figure 3-5b) of the ocular coils, we decided to narrow down the different types of ocular coils and continued with ocular coil 16/0.084/0.90 for further experimentation. This ocular coil was selected because it is rigid enough for manual handling and has a holding capacity for microspheres which appears sufficient for future drug loading. The ocular coil 16/0.084/0.90 was filled with PMMA microspheres (155 $\mu \mathrm{m} \pm 15 \mu \mathrm{m})$ using our proprietary funnel based technology, which was set to a filling of $3.0 \mathrm{mg}$. The distribution of filling is plotted in a histogram in figure 3-6 where after the histogram was analyzed for its goodness of fit towards a Gaussian distribution using the D'Agostino's $\mathrm{K}^{2}$ test. Filling of 103 ocular coils with PMMA microspheres was not normally distributed since a large amount of ocular coils $(n=68 / 103)$ were under- or overfilled $(P<0.0001)$. Filling of 103 ocular coils with PMMA microspheres was significantly different from the Gaussian distribution $(P<0.0001)$. The average filling was $3.01 \mathrm{mg} \pm 0.238 \mathrm{mg}$, which is in line with the set value of $3.0 \mathrm{mg}$ and within our defined tolerances $(2.9-3.1 \mathrm{mg})$.

\subsubsection{Microsphere escape from the ocular coil}

To investigate potential microsphere escape, ocular coil 16/0.084/0.90 was bent along the outer circumference of a glass disc with a diameter of $24 \mathrm{~mm}$. Figure 3-7a shows the distribution of the gap size between the turn-windings. The majority of gap sizes between turn-windings are between $4 \mu \mathrm{m}$ and $10 \mu \mathrm{m}$. The gap-size between turn-windings is not normally distributed (D'Agostino \& Pearson omnibus normality test, $\mathrm{K} 2=4.671$ with a $\mathrm{P}$ value of 0.0968 ). The mean calculated gap size between turn-windings was $7.93 \mu \mathrm{m}$ with a standard deviation of $\pm 3.66 \mu \mathrm{m}$ when bent along a $24 \mathrm{~mm}$ disc. The maximum measured gap size between turn-windings was 21.51 $\mu \mathrm{m}$ which was much larger than calculated from equation 7 .

When comparing measured to calculated values for the gap size between turnwindings, the measured gap size between the turn-windings is significantly larger than the calculated value ( $P$ value below 0.0001 ) as shown in figure $3-7 b$. The 
measured gap-size between the turn-windings was also significantly larger than the calculated maximum gap size between the turn-windings ( $P$ value equals 0.0045 ), calculated from equation 5 .

To experimentally test microsphere escape, a platform was designed to apply stretching forces simultaneously to the ocular coil while bending it. An increase in gap size between turn-windings was observed after applying both forces to the ocular coil; however, no escape of microspheres from the ocular coil was observed, not even at an elongation of $176.9 \%(L=44.16 \mathrm{~mm}$ ) (figure 3-7c). Under the microscope we observed that the microspheres clump together and stick to the coating of the wire as shown in figure 3-7d.

a

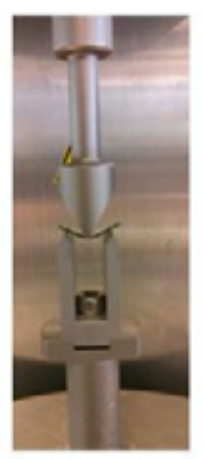

b

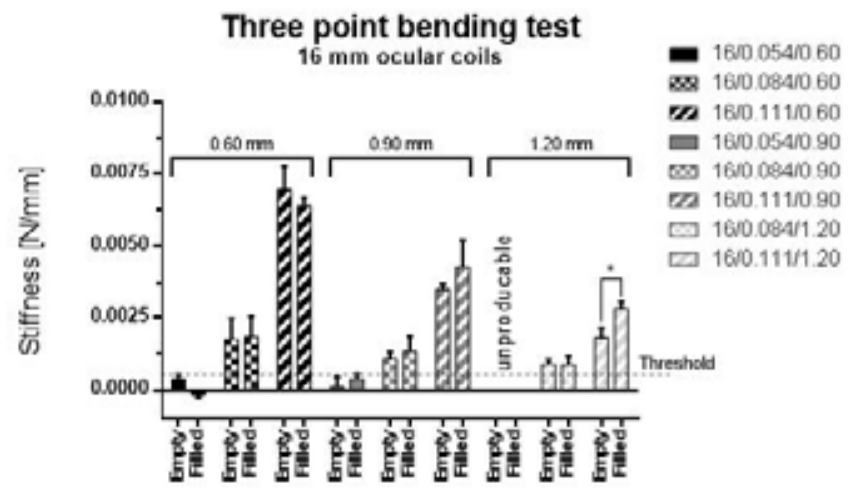

Figure 3-5. (a) Photo of a three point bending test and an ocular coil deforming under the influence of a vertical force originated from the indenter. (b) Results of the three point bending test plotted in columns. ${ }^{*} \mathrm{P}<0.05$ with an unpaired student $\mathrm{t}$-test, $\mathrm{n}=3$ ocular coils per measurement.

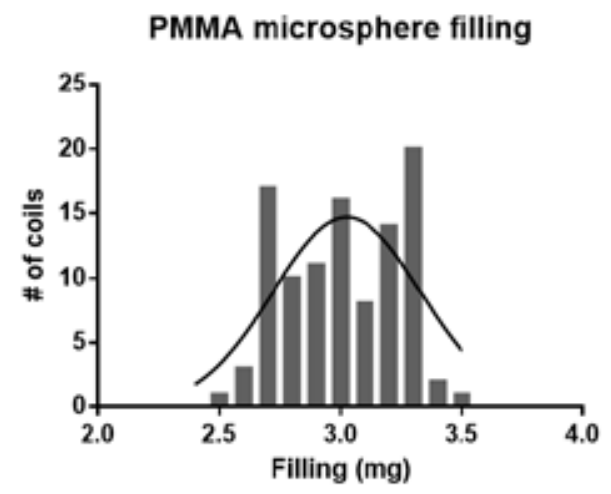

Figure 3-6. Distribution of filling of ocular coil 16/0.084/0.90 with PMMA microspheres, n=103 


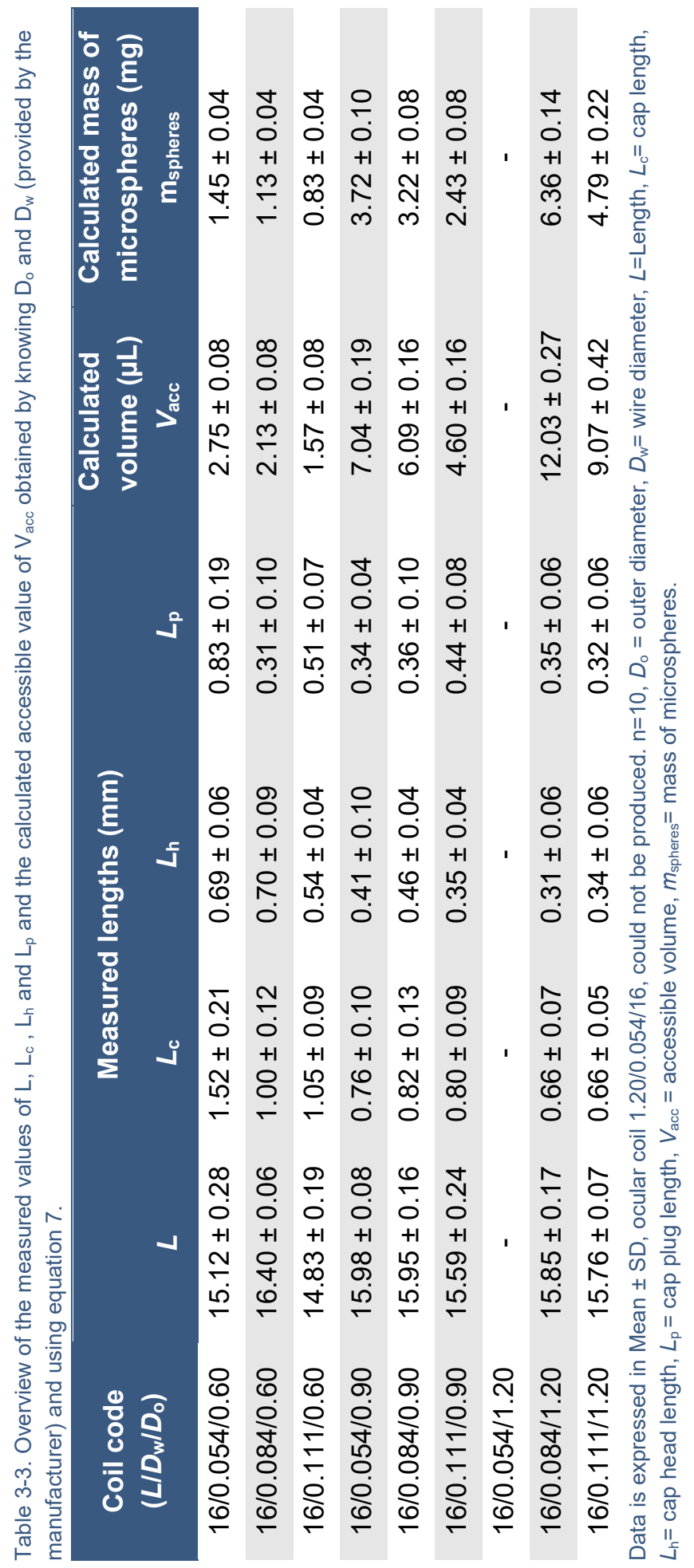




\subsubsection{Post mortem insertion of the ocular coil}

After insertion of the ocular coil in the lower conjunctival fornix of the head of a formalin fixed human cadaver a CT scan was made. First, the location of the ocular coil in the fornix was determined. The ocular coil is located deep in the fornix of the eye, in front of the inferior rectus muscle (figure 3-8a). Due to this position it is unlikely that the ocular coil interferes with eye movements and would be displaced. Second, the degree of bending of the ocular coil was measured. Both the ocular coils bent along the circumference of the eye (figure $3-8 b$ to $d$ ) with $\alpha=78.2^{\circ}$ (globe diameter of $23.7 \mathrm{~mm})$.
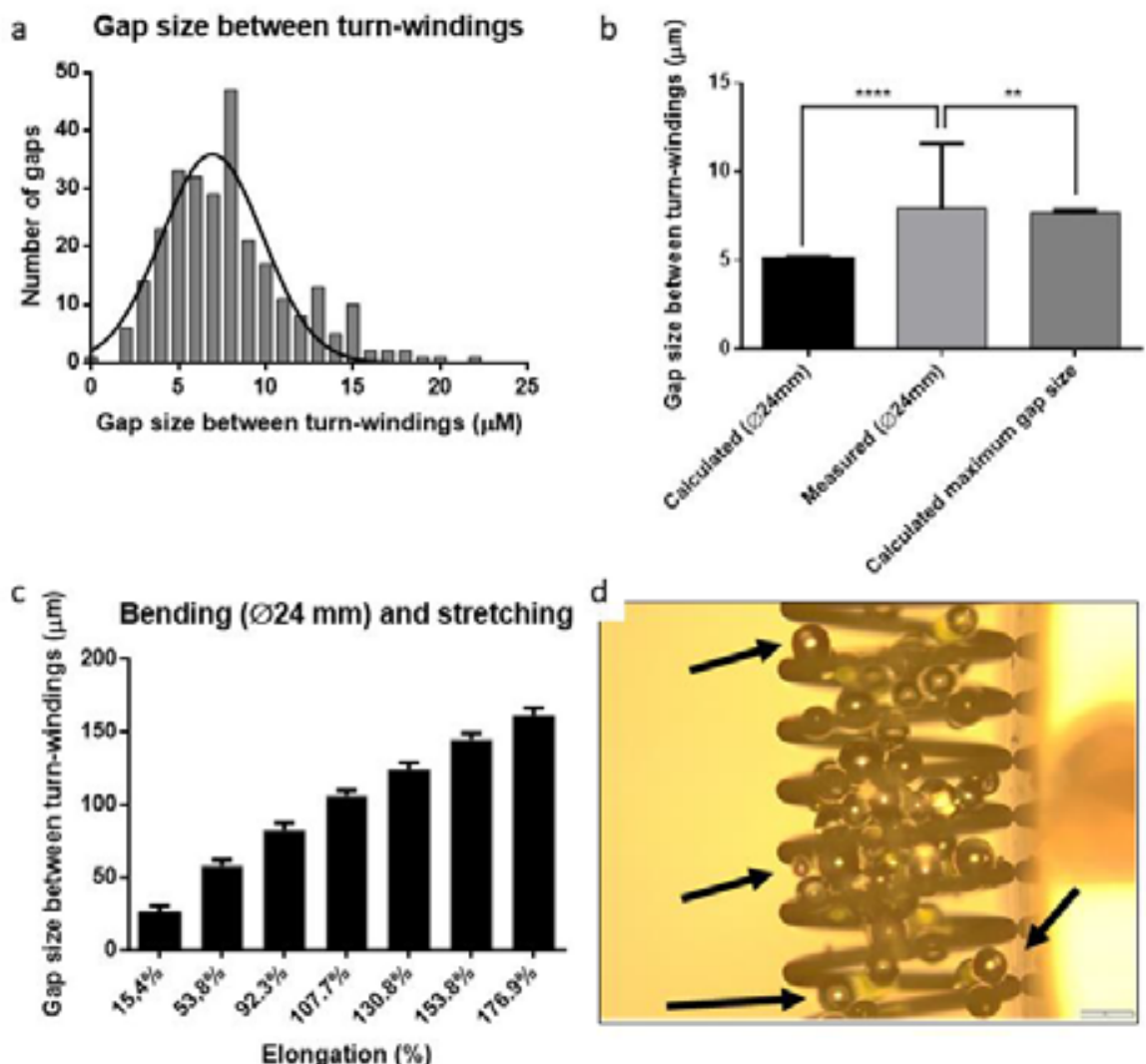

Figure 3-7. Gap size between turn-windings. (a) Histogram of the distribution of the gap sizes between the turn-windings ( $n=279$ gaps from 5 ocular coils) (b) Gap size between turn-windings calculated for a disc with $24 \mathrm{~mm}$ radius, the measured values and the calculated maximum gap size when the ocular coil bend $360^{\circ}$. ${ }^{* *} \mathrm{P}<0.01$ and ${ }^{* * * *} \mathrm{P}<0.0001$ with unpaired student $\mathrm{t}$-test calculated values were simulated with a Monte Carlo simulation with $n=2000$. (c) Bending and stretching an ocular coil, elongation of the ocular coil is shown in percentages on the x-axis. (d) Photo showing an ocular coil stretched at $176.9 \%$. Black arrows indicate microspheres with diameter smaller than the gap size between the turn-windings which did not escape. Scale bar is equal to $200 \mu \mathrm{m}$. 

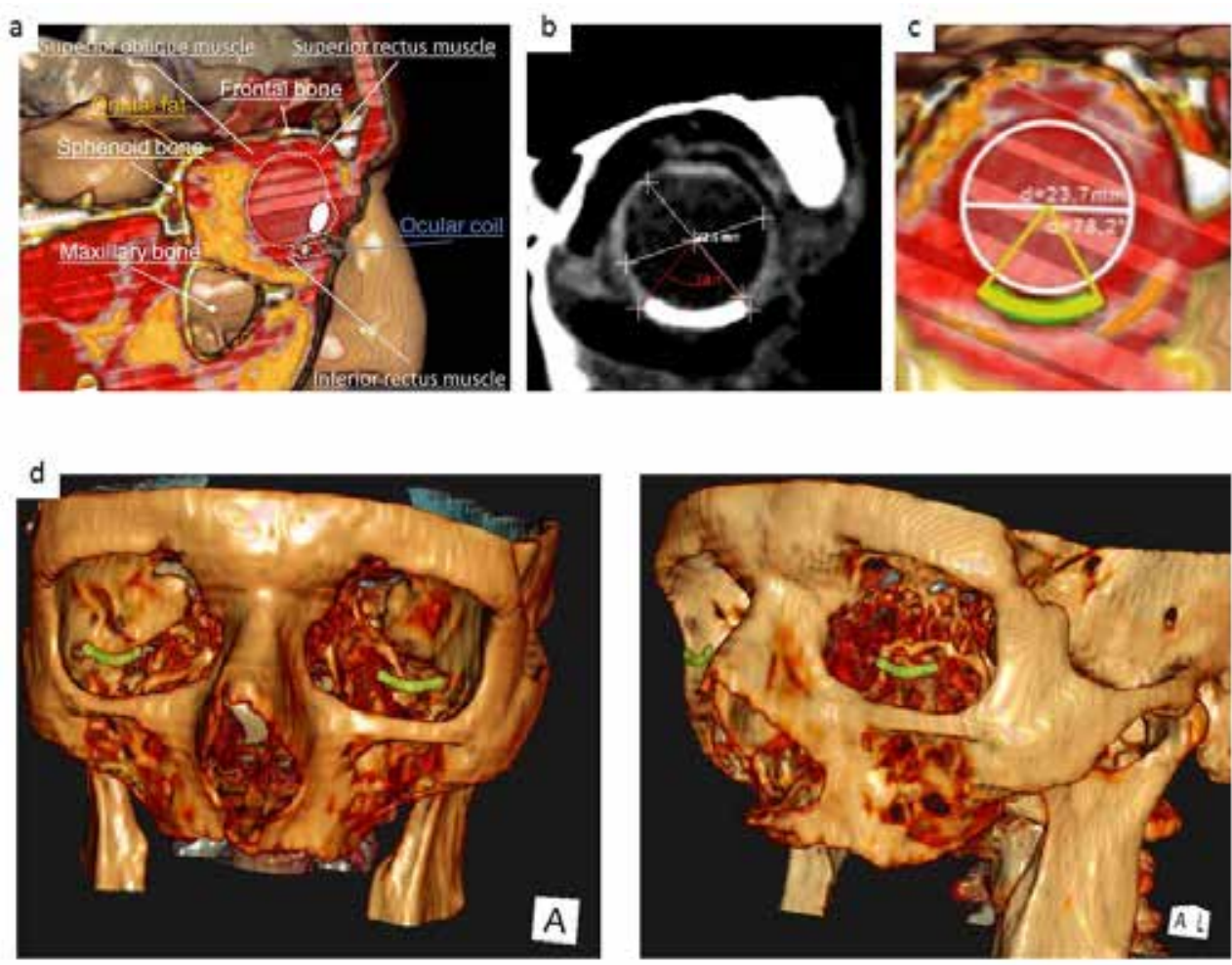

Figure 3-8. Location of the ocular coil in the conjunctival fornix of a formalin fixed human cadaver. (a) Sagittal section of the head with an ocular coil located in the fornix of the eye. (b and c) Bending of the ocular coil along the radius of the eye with calculated angle and size with Versalius 3D (b) and applied schematic drawing (c). (d) Overview photo of the ocular coil in the lower conjunctiva of the eye with all soft tissues removed (ocular coil is colored green).

\subsubsection{Drug release from the ketorolac-loaded, microsphere filled ocular coil}

Drug release studies revealed a high release of $48.7 \pm 5.4 \%$ in the first three days followed by a slow and sustained drug release period for up to 28 days (figure 3-9b). The highest drug release was observed between 1 and 4 hours after initiation of the study (figure 3-9a). During the first day, the ocular coil releases $274.7 \pm 43.5 \mu \mathrm{g}$. In the second day, $124.6 \pm 11.3 \mu \mathrm{g}$ was released. After one, two, three, and four weeks the release lowered to $14.6 \pm 1.7 \mu \mathrm{g}, 5.1 \pm 2 \mu \mathrm{g}, 3.1 \pm 1.1$, and $2.7 \pm 1.8 \mu \mathrm{g}$ per day, respectively (figure 3-9c).

Extraction of the microspheres from the ocular coil after 28 days of release showed that a total of $69.9 \pm 5.6 \%$ ketorolac was released from the ocular coil (figure 3-9b). In contrast to the sustained release profile of ketorolac from the ocular coil, eye drops delivered high concentrations of ketorolac per drop but decayed quickly, which results in the typical stair-like pattern of eye drops (figure 3-S2). 1 hour after 
application, the drug concentration from the ocular coil and eye drop are equally in our system. The concentration of ketorolac rises fast with each applied eye drop, whereas the ocular coil releases more gradually.
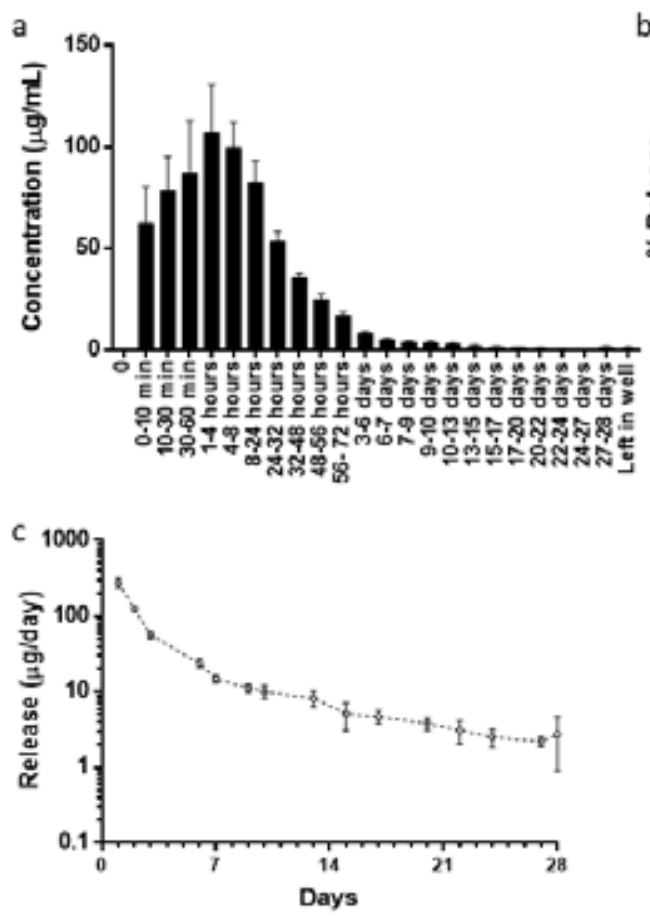

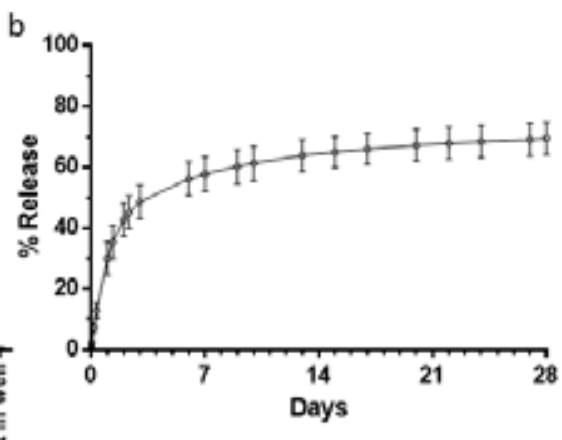

Figure 3-9. Drug release from the ocular coil. (a) Concentration of ketorolac $(\mu \mathrm{g} / \mathrm{mL})$, released by the ocular coil in the lacrimal system. (b) Cumulative release of ketorolac (\%) from the ocular coil over 28 days. (c) Release of ketorolac ( $\mu \mathrm{g} / \mathrm{day}$ ) from the ocular coil over 28 days. All data are reported as mean \pm standard deviation, $n=4$.

\subsection{Discussion}

Several characteristics of a novel ocular drug delivery system have been investigated in this study. By tuning the geometrical characteristics wire thickness and outer diameter, the holding capacity and flexibility of the ocular coil can be varied. A larger outer diameter of the ocular coil in combination with a smaller wire diameter results in a larger central cavity with a greater holding capacity the ocular coil. Differences in the accessible inner volume were observed between the measured and the calculated volume of the ocular coils 16/0.111/0.90 and $16 / 0.111 / 1.20$, where the measured accessible inner volume was slightly lower compared to the theoretically predicted accessible inner volume. This difference was probably caused by a slightly smaller outer diameter of the ocular coils in the 
received batch.

Next, we focused on ocular coil 16/0.084/0.90 for the following reasons. The ocular coils with an outer diameter of $0.60 \mathrm{~mm}$ might not hold enough microspheres for future drug release purposes. Ocular coils 16/0.84/1.20 and 16/0.54/0.90 were too flexible for proper handling. Ocular coils 16/0.111/1.20 and 16/0.111/0.90 showed lowered flexibility due to microsphere filling. To prevent the risk of an ocular coil which is too rigid; therefore, uncomfortable [19], we decided not to select these ocular coils for further investigations.

The ocular coil was filled with a volume based manual method, whereas mass is used to control whether the ocular coil is properly filled. In addition, there is some uncertainty in the volume fraction of particles in the filling, which might explain the deviation. Another important factor related to the variability in filling is that current batches of the ocular coil are filled manually. Eliminating the human interaction and replacing it by an automated system might lower the standard deviation.

Since microspheres might escape after filling the central coil cavity, the gap size between the turn-windings of the ocular coil was theoretically predicted and measured. The experiments showed that the calculated predictions are an underprediction of the gap size between the coil windings. The underestimation of the calculated gap size between the turn-windings can be explained by two reasons. Firstly, the calculations do not take into account, that ocular coils have caps of about $1 \mathrm{~mm}$ on both ends which are not capable of bending; due to the caps the ocular coil can only bend in the center. Secondly, an ocular coil with a smaller wire diameter in combination with a larger outer diameter stretches more easily than predicted. Due to the high flexibility of the ocular coil, a small extension occurred during the bending experiments, which could also explain the difference between the calculated and the measured values. However, no escape of the microsphere filling was observed, even when the ocular coil was extended to $179.9 \%$ of its original length (about $44.7 \mathrm{~mm}$ in length), thereby creating gaps between the turn-windings larger than $150 \mu \mathrm{m}$. Because the ocular coil was filled for $70 \%$ with microspheres, the microspheres were able to freely move in the ocular coil. We observed that the microspheres clumped together in the ocular coil and assume that the interaction of the coating with the PMMA microspheres induced a static interaction resulting in this effect; this hypothesis however, needs further investigation.

Eventually, the ocular coil was placed in the lower conjunctival fornix of a head of a human cadaver to investigate shape behavior and tissue interaction of the ocular coil. Bending of the ocular coil according to the anatomical configuration of the adnexa was visualized to gain insight in the contact between the ocular coil and the anatomical boundaries of the eye and shape behavior of the ocular coil. It was found that the ocular coil is located deep in the fornix, ventral from the muscles rectus inferior. The coil lies caudal from the lens in the fornix, where the bulbar conjunctiva 
transfers into the palpebral conjunctiva. Due to the position in the lower fornix, it seems unlikely that the ocular coil interferes with muscle movements and sensitive ocular tissues such as the cornea. CT imaging showed that the ocular coil fits well in the fornix and seems not to interfere with any critical ocular parts such as the cornea or muscles. However, we need to realize that the used human cadaver was formalin fixed, thus the tissue was much stiffer compared to a living human. We should also keep in mind that living humans have softer tissues so the ocular coil could be located even deeper into the fornix. As shown in prior studies by Pijls et al., use of an even more rigid ocular coil, although for a short period, was safe and did not cause irritation or discomfort.[20,21] In this study, two ocular coils were placed in the conjunctival fornix, without affecting comfort. However, an in-man study should confirm safety, comfort, and tolerability of the ocular coil in the lower conjunctival fornix for a prolonged time up to 28 days. Interestingly, a study conducted by Katz et al. showed that a rod shape ocular device is beneficial for fitting in the lower conjunctival fornix, compared to for instance oval or oblong shaped devices.[30] In our study, we compared $0.5 \%$ ophthalmic solution containing ketorolac (Acular ${ }^{\circledR}$ ) to a ketorolac loaded ocular coil in vitro. In this proof-of-concept study, loading of the ocular coil depended on the drug loading capacity of the microspheres and the volume of the inner lumen of the ocular coil. Drug release of the ketorolac-loaded microsphere filled ocular coil was tested in an in vitro lacrimal system.

In terms of drug release, two materials can influence drug release, the coils itself or the microencapsulating polymer. Previous studies suggest that the ocular coil itself does not hinder drug release, when fluorescein sodium and ciprofloxacin were used.[19]

Ketorolac tromethamine is a highly soluble compound with a $\log \mathrm{P}$ of 1.9.[31] We believe that the mechanism of drug release from the microspheres relies on delayed ketorolac dissolution that is the result of microencapsulation in hydrophobic PMMA. After increased release the first three days, the ocular coil has released about $50 \%$ of the drug (with an average release of $150 \mu \mathrm{g}$ per day). Drug release of the ocular coil slowly reduces to a more consistent dose over the following week (a total of $60 \%$ is released after 10 days). In the following 18 days, the ocular coil releases up to $69 \%$ of its drug content with an average release rate of $4.5 \pm 0.8 \mu \mathrm{g}$ per day. Release of the last 18 days tempers from $10.1 \pm 1.9 \mu \mathrm{g}$ per day on day 10 , to $2.2 \pm 0.3 \mu \mathrm{g}$ per day on day 28 . The drug-release profile with current microspheres suggests optimum usage for 14 days instead of 28 days. Furthermore, release experiments were performed at room temperature whereas the temperature of a human cul-de-sac lies between the $35^{\circ} \mathrm{C}$ and $36^{\circ} \mathrm{C}$.[32] Based on the glass transition temperature $\left(\mathrm{T}_{\mathrm{g}}\right)$ $\left(>85^{\circ} \mathrm{C}\right)$ and the heat distortion temperature (HDT) $\left(99^{\circ} \mathrm{C}-102^{\circ} \mathrm{C}\right)$ of PMMA, provided by the supplier, no temperature related release effect was anticipated, therefore, the experiments were performed at room temperature. 
This system was also used to evaluate drug release from eye drops. After 30 minutes, the same amount of drug was released from ocular coil compared to one eye drop (figure 3-S2). Afterwards, drug release from the eye drop increased gradually. However, this system does not consider pre-corneal loss of eye drops due to blinking or spillage during application. Hence, the entire eye drop $(50 \mu \mathrm{L}, \sim 0.25$ $\mathrm{mg}$ of ketorolac) can be found in the system and results in an over-estimation of the drug release. Since less than $5 \%$ of an eye drop actually penetrates into the anterior chamber.[14] The ocular coil does not affect the drug properties, such as solubility, permeability, metabolic stability, and transporter effects, since it is just a carrier. However, we created microspheres without additives, hence, the $\mathrm{pH}$ is not optimized, nor have we used penetration-enhancing stabilizers such as EDTA or benzalkonium chloride. This could affect ocular penetration as previously shown by Bertens et al. in post-mortem pig eyes.[33] To investigate the pharmacokinetics and the effectivity of the released drug doses from the ocular coil, in vivo animal experiments are planned.

\subsection{Conclusion}

In this study we demonstrated an optimization pathway of an ocular coil for drug delivery purposes. Due to the coil design drug loaded microspheres can be filled into the inner lumen of the ocular coil without affecting its flexibility. The ocular coil seems a promising carrier for ophthalmic drugs delivery in the early postoperative time period. To investigate safety and comfort of the ocular coil, a first-in-man study is planned (NCT03488017). In addition, experimental animal drug delivery studies will be performed.

\subsection{References}

1.

Stratagem IP Management Limited, The Ophthalmology Market 2015, Stratagem Intellectual Property Management Limited: Online (18-Sep-2018), http://www.evaluationsonline.org.uk/evaluations/Documents.do?action=download\&id=775\&ui =basic. p. 46 .

2. Gaudana, R., Ananthula, H.K., Parenky, A., and Mitra, A.K., Ocular drug delivery. AAPS J, 2010. 12(3): p. 348-360.

3. Hughes, P.M., Olejnik, O., Chang-Lin, J.E., and Wilson, C.G., Topical and systemic drug delivery to the posterior segments. Adv Drug Deliv Rev, 2005. 57(14): p. 2010-2032.

4. Urtti, A. and Salminen, L., Concentration-dependent precorneal loss of pilocarpine in rabbit eyes. Acta Ophthalmol (Copenh), 1985. 63(5): p. 502-506.

5. Urtti, A. and Salminen, L., Minimizing systemic absorption of topically administered ophthalmic drugs. Surv Ophthalmol, 1993. 37(6): p. 435-456.

6. Del Amo, E.M., Rimpela, A.K., Heikkinen, E., Kari, O.K., Ramsay, E., Lajunen, T., Schmitt, M., Pelkonen, L., Bhattacharya, M., Richardson, D., Subrizi, A., Turunen, T., Reinisalo, M., Itkonen, J., Toropainen, E., Casteleijn, M., Kidron, H., Antopolsky, M., Vellonen, K.S., Ruponen, M., and Urtti, A., Pharmacokinetic aspects of retinal drug delivery. Prog Retin Eye Res, 2017. 57: p. 134-185. 
7. Pelkonen, L., Tengvall-Unadike, U., Ruponen, M., Kidron, H., Del Amo, E.M., Reinisalo, M., and Urtti, A., Melanin binding study of clinical drugs with cassette dosing and rapid equilibrium dialysis inserts. Eur J Pharm Sci, 2017. 109: p. 162-168.

8. Farkouh, A., Frigo, P., and Czejka, M., Systemic side effects of eye drops: a pharmacokinetic perspective. Clin Ophthalmol, 2016. 10: p. 2433-2441.

9. Eaton, A.M., Gordon, G.M., Konowal, A., Allen, A., Allen, M., Sgarlata, A., Gao, G., Wafapoor, H., and Avery, R.L., A novel eye drop application monitor to assess patient compliance with a prescribed regimen: a pilot study. Eye (Lond), 2015. 29(10): p. 1383-1391.

10. Mohindroo, C., Ichhpujani, P., and Kumar, S., How 'Drug Aware' are our Glaucoma Patients? J Curr Glaucoma Pract, 2015. 9(2): p. 33-37.

11. Newman-Casey, P.A., Robin, A.L., Blachley, T., Farris, K., Heisler, M., Resnicow, K., and Lee, P.P., The Most Common Barriers to Glaucoma Medication Adherence: A Cross-Sectional Survey. Ophthalmology, 2015. 122(7): p. 1308-1316.

12. Nordmann, J.P., Baudouin, C., Renard, J.P., Denis, P., Lafuma, A., Laurendeau, C., Jeanbat, V., and Berdeaux, G., Measurement of treatment compliance using a medical device for glaucoma patients associated with intraocular pressure control: a survey. Clin Ophthalmol, 2010. 4: p. 731-739.

13. An, J.A., Kasner, O., Samek, D.A., and Levesque, V., Evaluation of eyedrop administration by inexperienced patients after cataract surgery. J Cataract Refract Surg, 2014. 40(11): p. 18571861.

14. Bertens, C.J.F., Gijs, M., van den Biggelaar, F., and Nuijts, R., Topical drug delivery devices: A review. Exp Eye Res, 2018. 168: p. 149-160.

15. Brandt, J.D., DuBiner, H.B., Benza, R., Sall, K.N., Walker, G.A., Semba, C.P., and Collaborators, Long-term Safety and Efficacy of a Sustained-Release Bimatoprost Ocular Ring. Ophthalmology, 2017. 124(10): p. 1565-1566.

16. Brandt, J.D., Sall, K., DuBiner, H., Benza, R., Alster, Y., Walker, G., Semba, C.P., and Collaborators, Six-Month Intraocular Pressure Reduction with a Topical Bimatoprost Ocular Insert: Results of a Phase II Randomized Controlled Study. Ophthalmology, 2016. 123(8): p. 1685-1694.

17. Walters, T., Endl, M., Elmer, T.R., Levenson, J., Majmudar, P., and Masket, S., Sustainedrelease dexamethasone for the treatment of ocular inflammation and pain after cataract surgery. J Cataract Refract Surg, 2015. 41(10): p. 2049-2059.

18. Torkildsen, G., Abelson, M.B., Gomes, P.J., McLaurin, E., Potts, S.L., and Mah, F.S., VehicleControlled, Phase 2 Clinical Trial of a Sustained-Release Dexamethasone Intracanalicular Insert in a Chronic Allergen Challenge Model. J Ocul Pharmacol Ther, 2017. 33(2): p. 79-90.

19. Pijls, R.T., Cruysberg, L.P., Nuijts, R.M., Dias, A.A., and Koole, L.H., Capacity and tolerance of a new device for ocular drug delivery. Int J Pharm, 2007. 341(1-2): p. 152-161.

20. Pijls, R.T., Hanssen, H.H., Nuijts, R.M., Daube, G.W., and Koole, L.H., In vivo tolerance and kinetics of a novel ocular drug delivery device. J Control Release, 2006. 116(2): p. e47-49.

21. Pijls, R.T., Hanssen, H.H., Nuijts, R.M., and Koole, L.H., Flexible coils with a drug-releasing hydrophilic coating: a new platform for controlled delivery of drugs to the eye? Biomed Mater Eng, 2004. 14(4): p. 383-393.

22. Pijls, R.T., Sonderkamp, T., Daube, G.W., Krebber, R., Hanssen, H.H., Nuijts, R.M., and Koole, L.H., Studies on a new device for drug delivery to the eye. Eur J Pharm Biopharm, 2005. 59(2): p. 283-288.

23. Holden, B.A., The Glenn A. Fry Award lecture 1988: the ocular response to contact lens wear. Optom Vis Sci, 1989. 66(11): p. 717-733.

24. American Academy of Ophthalmology., Fundamentals and principles of ophthalmology, in Basic and clinical science course. 2014, American Academy of Ophthalmology: San Francisco, CA. p. 37.

25. Govender, T., Stolnik, S., Garnett, M.C., Illum, L., and Davis, S.S., PLGA nanoparticles prepared by nanoprecipitation: drug loading and release studies of a water soluble drug. J Control Release, 1999. 57(2): p. 171-185.

26. Zhang, P., Liu, X., Hu, W., Bai, Y., and Zhang, L., Preparation and evaluation of naringeninloaded sulfobutylether-beta-cyclodextrin/chitosan nanoparticles for ocular drug delivery. Carbohydr Polym, 2016. 149: p. 224-230.

27. Jaeger, H.M. and Nagel, S.R., Physics of the granular state. Science, 1992. 255(5051): p. 15231531.

28. Torquato, S., Truskett, T.M., and Debenedetti, P.G., Is random close packing of spheres well defined? Phys Rev Lett, 2000. 84(10): p. 2064-2067. 
Chapter 3

29. Zhou, Z., Anselmo, A.C., and Mitragotri, S., Synthesis of protein-based, rod-shaped particles from spherical templates using layer-by-layer assembly. Adv Mater, 2013. 25(19): p. 27232727.

30. Katz, I.M. and Blackman, W.M., A soluble sustained-release ophthalmic delivery unit. Am J Ophthalmol, 1977. 83(5): p. 728-734.

31. PubChem. Ketorolac (Compound): Online (accessed: 12-feb-2020), https://pubchem.ncbi.nlm.nih.gov/compound/Ketorolac\#section=Canonical-SMILES.

32. García-Porta, N., Gantes-Nuñez, F.J., Tabernero, J., and Pardhan, S., Characterization of the ocular surface temperature dynamics in glaucoma subjects using long-wave infrared thermal imaging. J. Opt. Soc. Am. A, 2019. 36(6): p. 1015-1021.

33. Bertens, C.J.F., Zhang, S., Erckens, R.J., van den Biggelaar, F., Berendschot, T., Webers, C.A.B., Nuijts, R., and Gijs, M., Confocal Raman spectroscopy: Evaluation of a non-invasive technique for the detection of topically applied ketorolac tromethamine in vitro and in vivo. Int $\mathrm{J}$ Pharm, 2019. 570: p. 118641. 


\subsection{Supplementary figures}

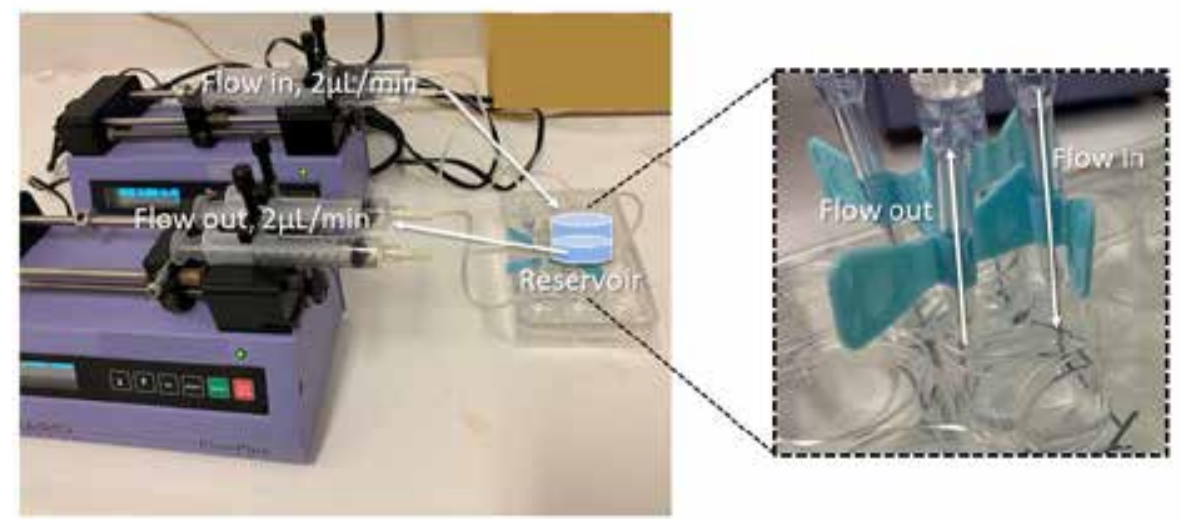

Figure 3-S1. Overview of the in vitro lacrimal system using two syringe pumps pumping a constant flow of $2 \mu \mathrm{L} / \mathrm{min}$ in and out a reservoir.

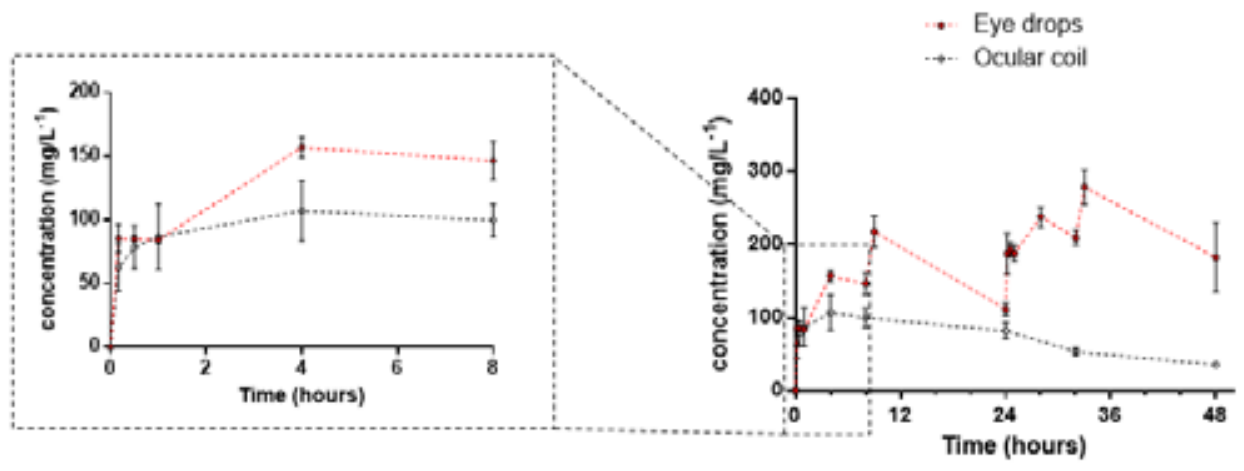

Figure 3-S2. Drug release from the ocular coil compared to eye drops for the first 48 hours, both measured in the in vitro lacrimal system. All data are reported as mean \pm standard deviation, $n=4$ 


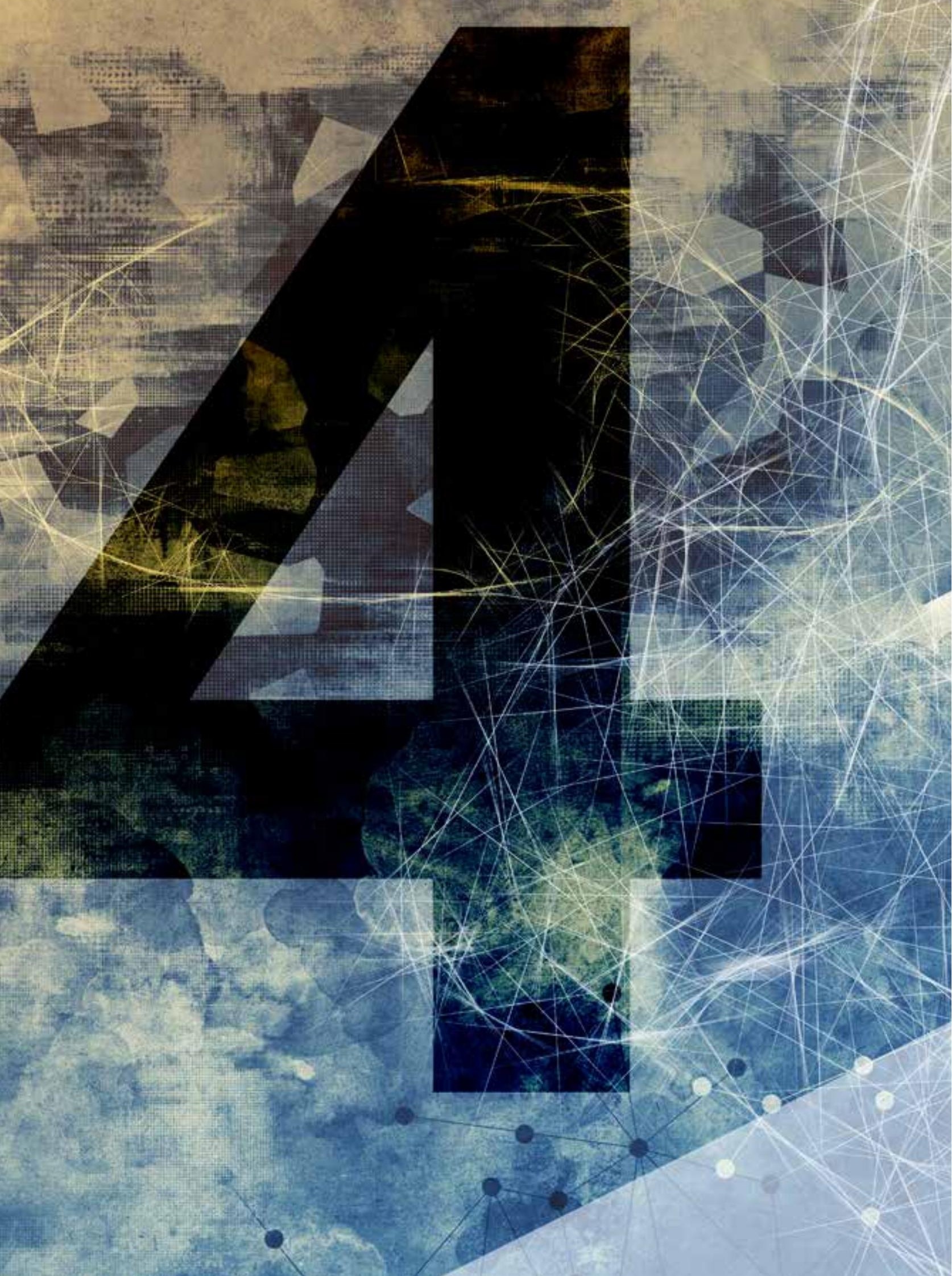


Chapter 4.

Phamacokinetics and efrcacy of a

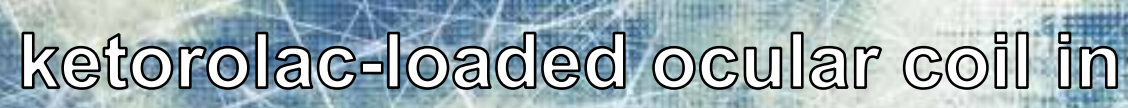

New Zealand Write rabbits

Christian J.F. Bertens, Marlies Gijs, Aylvin A. Dias, Frank J.H.M. van den Biggelaar, Arkasubhra Ghosh, Swaminathan Sethu, and Rudy M.M.A. Nuijts

Drug Delivery, January 2021, 28:1, 400-407

DOI: $10.1080 / 10717544.2021 .1883157$ 


\section{Abstract}

Eye drops are considered standard practice for the delivery of ocular drugs. However, low patient compliance and low drug levels compromise its effectiveness. Our group developed a ketorolac-loaded ocular coil for sustained drug delivery up to 28 days. The aim of this study was to gain insight into the pharmacokinetics and efficacy of the ocular coil.

The pharmacokinetics of the ketorolac-loaded ocular coil versus eye drops were tested in New Zealand White rabbits by repetitive sampling for 28 days.

Efficacy of the ocular coil was also tested in New Zealand White rabbits. Ocular inflammation was induced where after the ocular coil was inserted, or eye drops, or no treatment was provided. The total protein concentration and cytokine levels were measured in tears, aqueous humor, and plasma at $4 \mathrm{~h}, 8 \mathrm{~h}, 24 \mathrm{~h}, 4 \mathrm{~d}, 7 \mathrm{~d}, 14 \mathrm{~d}, 21 \mathrm{~d}$, and 28d.

$4 \mathrm{~h}$ after inserting the ocular coil in the eye, ketorolac levels in aqueous humor and plasma were higher in the ocular coil group than in the eye drop group. Ketorolac released from the ocular coil could be detected up to $28 \mathrm{~d}$ in tears, up to $4 \mathrm{~d}$ in aqueous humor and up to $24 \mathrm{~h}$ in plasma.

After inducing inflammation, both the ocular coil and eye drops were able to suppress prostaglandin E2, TNFa and IL-6 levels in aqueous humor and plasma as compared to the group that received no treatment. To conclude, the ocular coil facilitated a sustained release of the drug and showed similar therapeutic benefit in suppressing post-operative inflammation as eye drops.
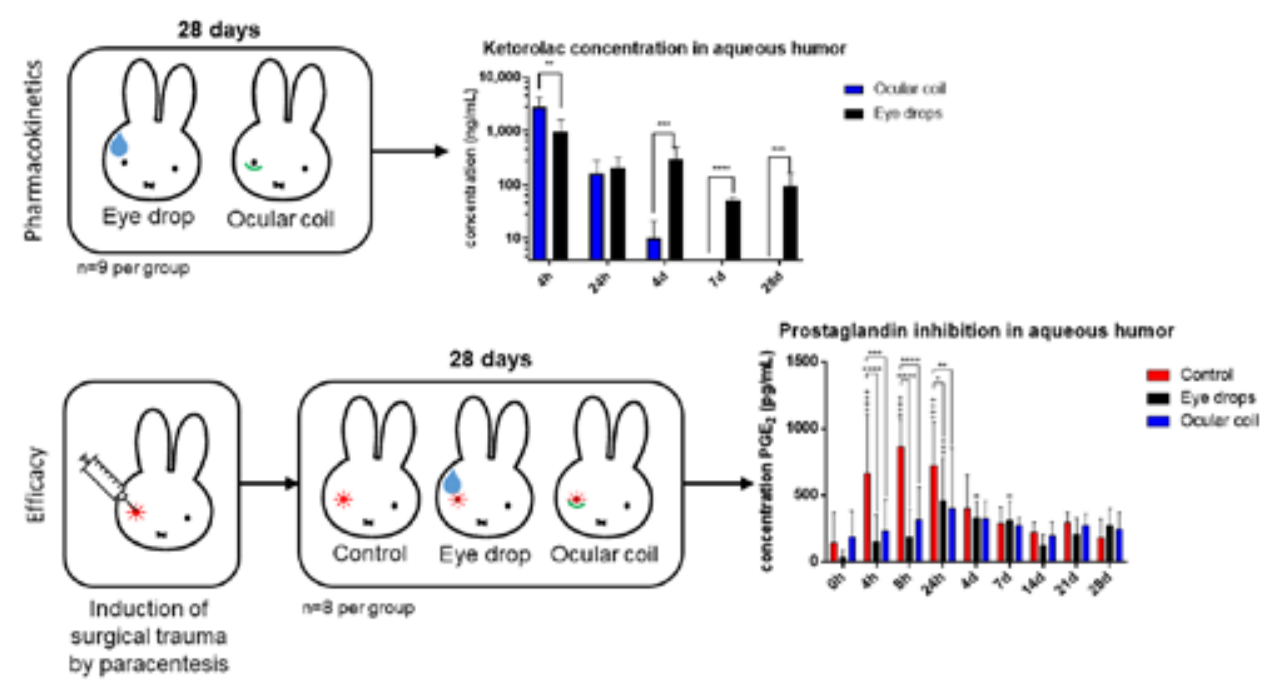

Graphical abstract 


\subsection{Introduction}

Topical administration of eye drops is the most commonly prescribed treatment strategy in the prevention and treatment of ocular disorders.[1] Despite the achieved therapeutic concentrations in anterior segment tissues, eye drops have significant disadvantages. A short duration of action, high peak drug concentrations, and considerable systemic absorption of the drug are several important shortcomings of eye drops.[2] In order to maintain minimal effective concentrations (MEC), drugs need to be dosed frequently. However, it is known that patient compliance (the degree to which a patient correctly follows medical advice) of eye drops is low.[3-6] Frequently reported reasons for non-compliance include forgetfulness $(26.7 \%$ of patients treated with eye drops), limited access to eye drops (20\%), and insufficient ability to properly self-instil the eye drops (16.2\%).[4] As a result of low compliance, the effectivity of the prescribed therapy is compromised.

To improve drug delivery and bypass patient compliance issues, injections (subconjunctival, subtenon, intracameral, intravitreal) into the target site can be used. However, injections only deliver a single (high) dose of drugs at a single time point to the affected eye. Furthermore, injections are invasive and can be accompanied with complications or side effects. Therefore, new methods for ocular drug delivery are essential within the ophthalmic field.

In addition to in vitro drug release studies, in vivo studies are needed to determine the pharmacokinetics, pharmacodynamics, and the MEC of the delivered drugs in a complete system. Based on these values, application regimes can be optimized and safety of the drugs (and the additives) can be assured.

To improve ocular drug delivery, our group developed an ocular coil that can be placed in the lower conjunctival fornix.[7-11] The ocular coil consists of a coiled and coated wire, closed on both extremities with a dome-shaped cap. The ocular coil is filled with a non-steroidal anti-inflammatory drug (NSAID), ketorolac tromethamine, containing microspheres in its inner lumen to serve as a slow-release drug delivery device. In our previous study, we show in vitro release of ketorolac for 28 days from the ocular coil.[11]

In this preclinical study, we investigate the pharmacokinetics of a ketorolac-loaded ocular coil, and tested its efficacy of suppressing inflammation after surgical trauma in New Zealand White rabbits. Surgical trauma was mimicked by a paracentesis of the anterior chamber.

\subsection{Materials and methods}

\subsubsection{The ocular coil}

The technical details and in vitro release kinetics have been previously described.[11] Briefly, ocular coils (16 mm long, wire thickness of $0.084 \mathrm{~mm}$ with an 
outer diameter of $0.90 \mathrm{~mm}$ ) were ordered from EPflex (Dettingen an der Erms, DE). The ocular coils were manually filled with $3 \mathrm{mg}$ ketorolac entrapped poly-methyl methacrylate (PMMA, Mn $\approx 43 \mathrm{~kg} / \mathrm{mol}$ ) microspheres ( $26.5 \mathrm{wt} \%$ drug loading) 150 $\mu \mathrm{m} \pm 10 \mu \mathrm{m}$ in diameter. Hereafter, the ocular coil was closed on both extremities with a dome-shaped UV-curable acrylate urethane cap to soften its extremities while maintaining the drug-eluting matrix inside. The in vitro release kinetic study showed that a total of $69.9 \pm 5.6 \%(0.795 \pm 0.063 \mathrm{mg}$ ketorolac $)$ of the loaded ketorolac was released in 28 days. In the first three days, a high (burst) release of approximately $50 \%$ of ketorolac was observed followed by a more gradual release up to 28 days.

\subsubsection{Ethics}

All animal procedures were conducted according to the Association for Research in Vision and Ophthalmology (ARVO). Statement for the Use of Animals in Ophthalmic and Visual Research and the Guidelines of the Central Laboratory Animal Facility of Maastricht University. All protocols were approved by the Central Authority for Scientific Procedures on Animals (CCD, Den Haag, the Netherlands) and were in accordance with the European Guidelines (2010/63/EU).

\subsubsection{Animals}

Adult New Zealand White (NZW) rabbits $(2.0 \mathrm{~kg}-2.5 \mathrm{~kg}$, males and females, strain: Hsdlf:NSW) were ordered from Envigo (Horst, NL) and housed in group housing, males and females separated with a maximum of five rabbits per cage (size: $\left.4 \mathrm{~m}^{2}\right)$. The rabbits had ad libitum access to water (regular tap water) and dried animal chow (200gr per animal). After arrival, the animals received one week of acclimatization to the new environment.

During the first experimental procedure (stitching), rabbits were intramuscularly (IM) sedated using ketamine $(50 \mathrm{mg} / \mathrm{kg}$ ) (Alfasan Nederland BV, Woerden, NL) and midazolam $(0.5 \mathrm{mg} / \mathrm{kg}$ ) (Actavis, Dublin, IR). Additionally, they received topical anesthesia using MINIMS $®$ Oxybuprocaine hydrochloride (Bausch \& Lomb Pharma, Brussels, BE). Because of the nictitating membrane in rabbits, the ocular coil was stitched into the conjunctival fornix using nylon 8-0 12" stitches (Alcon Inc., Genève, $\mathrm{CH}$ ). The first stitch was placed centrally, followed by one stitch nasally and one stitch temporally from the first stitch (figure 4-1). The other groups also received three stitches without an ocular coil.

During the follow-up moments, rabbits were sedated using medetomidine $(1 \mathrm{mg} / \mathrm{kg})$ (A.S.T. Farma BV, Oudewater, NL). After the final sampling at day 28 , the rabbits were euthanized using $20 \%$ sodium pentobarbital $\left(200 \mathrm{mg} / \mathrm{kg}\right.$ ) (Euthasol ${ }^{\circledR}$, Alfasan Nederland BV, Woerden, NL) intravenously (IV) injected. 


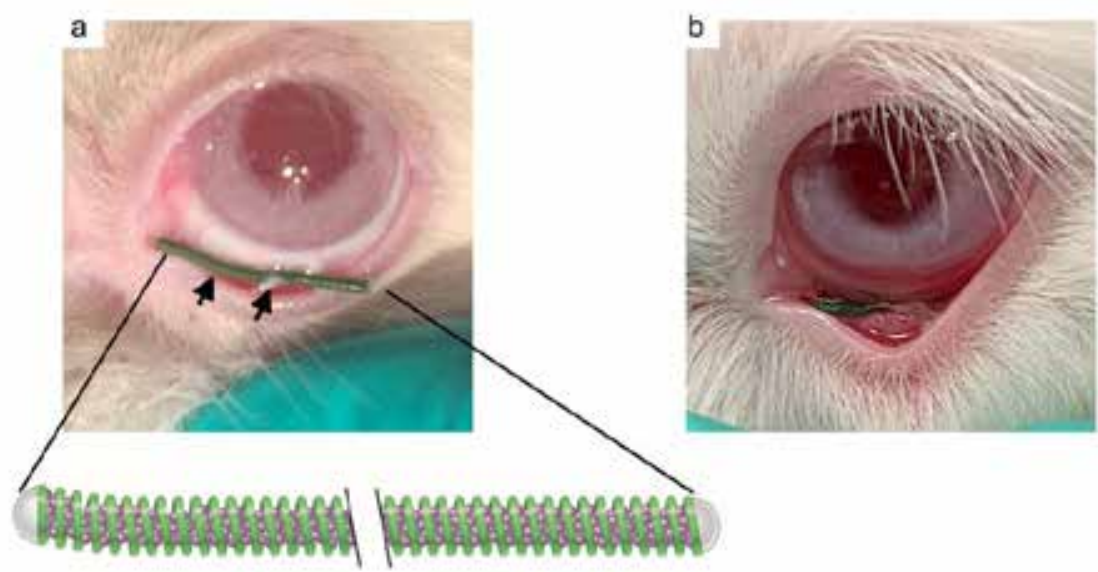

Figure 4-1. a) Location of the ocular coil in the conjunctival fornix during the stitching procedure. The arrows indicate two of the three stitches. The magnification below shows a representation of the ocular coil and its microsphere filling. b) Location of the ocular coil in the conjunctival inferior fornix during normal wear.

\subsubsection{Treatment groups}

Rabbits from the ocular coil group received one ketorolac-loaded ocular coil in the conjunctival fornix of their right eye. The eye drop group received $50 \mu \mathrm{L}$ ketorolac ophthalmic solution (Acular ${ }^{\mathrm{Tm}}, 0.5 \%$ ophthalmic ketorolac solution $(5 \mathrm{mg} / \mathrm{mL}$ ), Allergan, Dublin, IR) in the conjunctival fornix of their right eye immediately, 4 hours, and 10 hours after the stitching procedure. During the following 27 days, these rabbits received eye drops three times daily. Rabbits from the control group did not receive any treatment.

Samples of aqueous humor, tears, and blood from the rabbits of the pharmacokinetic study were drawn at 4 and 24 hours, and at day 4, day 7 , and day 28 after stitching. Samples of aqueous humor, tears and blood of the rabbits from the efficacy study were drawn at 4,8 , and 24 hours, and at days $4,7,14,21$, and 28 after trauma induction.

\subsubsection{Induction of inflammation}

Inflammation was induced by removing a large volume (approximately 150-175 $\mu \mathrm{L}$ ) aqueous humor via a corneal paracentesis as previously described by Unger et al. using a $1 \mathrm{~mL}$ insulin syringe and a 29G needle (Becton Dickinson BV, Vianen, $\mathrm{NL})$.[12] Caution was taken not to touch the lens or iris during the procedure. The collected aqueous humor was stored in a $1.5 \mathrm{~mL}$ Eppendorf vial at $-80^{\circ} \mathrm{C}$. 


\subsubsection{Sample collection}

Tears were sampled from the right eye of the rabbits using Schirmers' TEARstrips (Contacare Ophthalmics \& Diagnostics, Gujarat, IN). The Schirmer's strips were placed in the inferior conjunctival fornix for 5 minutes or until complete absorption. Hereafter, the Schirmer's strips were placed in a $1.5 \mathrm{~mL}$ Eppendorf vial and frozen at $-80^{\circ} \mathrm{C}$ until further treatment. Hereafter, about $3 \mathrm{~mL}$ blood was collected via the marginal ear vein into a $5 \mathrm{~mL}$ EDTA vacuette tubes (VWR, Amsterdam, NL). After sampling, the vacuette tubes were centrifuged $1500 \mathrm{G}$ for 10 minutes at $4^{\circ} \mathrm{C}$. Plasma was gently pipetted off and frozen at $-80^{\circ} \mathrm{C}$. This was followed by anterior chamber paracentesis. The paracentesis was performed with a $1 \mathrm{~mL}$ insulin syringe (29G) (Becton Dickinson BV, Vianen, NL). During the sampling, a small volume (approximately $50 \mu \mathrm{L}$ ) aqueous humor was drawn and frozen at $-80^{\circ} \mathrm{C}$ until further use. Caution was taken to avoid touching the lens or iris.

\subsubsection{Protein and ketorolac extraction from tears}

Tears were extracted from the Schirmer's TEAR strips as described earlier by Sharma et al.[13] Briefly, the strips were cut into $1 \mathrm{~mm}$ pieces and soaked in $200 \mu \mathrm{L}$ PBS ( $\mathrm{pH} 7.4$ ) for protein extraction, or in $200 \mu \mathrm{L}$ methanol (99.9\% pure, HPLC grade) (VWR, Amsterdam, NL) for ketorolac extraction. This was agitated at $900 \mathrm{rpm}$ (Thermomixer, Eppendorf, Hamburg, DE) at $4^{\circ} \mathrm{C}$ for 90 minutes. Paper was filtered off and collected tear fluid was used for further experiments. The measured concentrations (ketorolac, proteins, and cytokines) were corrected for the tear migration length and dilution in order to obtain the corrected concentration per milliliter.

\subsubsection{Ketorolac detection}

Aqueous humor and plasma were diluted four times with methanol $(99.9 \%$ pure, HPLC grade) (VWR, Amsterdam, NL) and centrifuged for 5 minutes at 15,000G at $4^{\circ} \mathrm{C}$ to remove proteins. Methanol extracted tears were used without further dilution. The samples were analyzed by HPLC (Agilent 1260 infinity series with EZchrom software, Agilent inc., Santa Clara, CA, USA). Analysis was done according to the US Pharmacopeia[14], using an elution time of 20 minutes and injection volume of $10 \mu \mathrm{L}$, peak UV-detection at $313 \mathrm{~nm}$ on a symmetry C18 column $(300 \AA, 5 \mu \mathrm{m}, 4.6$ $\mathrm{mm} \times 250 \mathrm{~mm}$; \#WAT106151, Waters corp., Milford, MA, USA) with a symmetry C8 VanGuard pre-column (100A, $5 \mu \mathrm{m}, 3.9 \mathrm{~mm} \times 5 \mathrm{~mm}$, 3/pkg, \#186007739, Waters corp., Milford, MA, USA). Ketorolac had a retention time of 10.5 minutes, a limit of detection (LOD) of $4 \mathrm{ng} / \mathrm{mL}$, and a limit of quantification (LOQ) of $10 \mathrm{ng} / \mathrm{mL}$.[15] All samples were analyzed in duplicate. 


\subsubsection{Total protein and inflammatory factor determination}

The total protein concentration was determined using BCA protein assay (ThermoFisher scientific, Waltham, MA, USA). Enzyme-linked immunosorbent assays (ELISAs) were used for the determination of prostaglandin $\mathrm{E}_{2}\left(\mathrm{PGE}_{2}\right)$, tumor necrosis factor $\alpha$ (TNFa), interleukin (IL)-6, and IL-1 $\beta$ concentration in aqueous humor, plasma, and tears. $\mathrm{PGE}_{2}$ was determined using the Biotrak ${ }^{\mathrm{TM}}$ EIA kit (\#GERPN222, Merck KGaA, Darmstadt, DE). Samples were diluted 1:4 using assay buffer and a total of $50 \mu \mathrm{L}$ diluted sample was loaded per well. TNFa, IL-6, and IL$1 \beta$ were determined using R\&D systems DuoSet (\#DY5670, \#DY7984, \#DY7464, R\&D Systems, Inc., McKinley Place, MN, USA). Samples were also diluted 1:4 using reagent diluent and $50 \mu \mathrm{L}$ diluted sample was loaded per well. The assays were performed in singlicate due to limited sample volume.

\subsubsection{Statistical analysis}

Differences in drug concentration between treatment groups were tested using unpaired student t-test. Samples below the detection limit of ketorolac $(4 \mathrm{ng} / \mathrm{mL})$ were set to a value of $4 \mathrm{ng} / \mathrm{mL}$.

For the protein and cytokine assays, outliers were excluded using the robust regression and outlier removal (ROUT) method with a $Q$ of $1 \%$.[16] Differences in the total protein concentrations between treatment groups were tested for each time point using Tukey's single-step multiple comparison procedure. Furthermore, Dunnett's test was performed for pairwise comparisons of multiple time point to baseline.

All tests were performed using GraphPad Prism version 8 (GraphPad Software inc. San Diego, CA, USA).

\subsection{Results}

\subsubsection{Pharmacokinetics of the ocular coil versus eye drops}

The pharmacokinetics of the ketorolac-loaded ocular coil was evaluated by measuring the ketorolac concentration in tears, aqueous humor, and plasma at multiple time points (figure 4-2). The ketorolac concentration released by the ocular coil at 4 hours in tears, aqueous humor, as well as plasma was significantly higher compared to the concentration delivered by the eye drops.

At 4 hours, the ketorolac tear concentration in the ocular coil group was 28 times higher than in the eye drop group $(950 \pm 782 \mu \mathrm{g} / \mathrm{mL}$ compared to $34 \pm 32 \mu \mathrm{g} / \mathrm{mL}$, respectively, $p=0.003)$. At 24 hours, the tear ketorolac concentration in the ocular coil group was about 9 times higher than in the eye drop group $(397 \pm 348 \mu \mathrm{g} / \mathrm{mL}$ compared to $44 \pm 17 \mu \mathrm{g} / \mathrm{mL}$ respectively, $\mathrm{p}=0.008$ ). During the first 4 days, the ketorolac concentration in tears (figure 4-2a) in the ocular coil group was higher than 
in the eye drop group. At day 7 and 28, the concentration in tears in the ocular coil group was equal to that of eye drops $(39 \pm 14 \mu \mathrm{g} / \mathrm{mL}$ at day 7 and $19 \pm 12 \mu \mathrm{g} / \mathrm{mL}$ at day 28 compared to $44 \pm 35 \mu \mathrm{g} / \mathrm{mL}$ at day 7 and $13 \pm 20 \mu \mathrm{g} / \mathrm{mL}$ at day 28 for the ocular coil group and the eye drop group, respectively).

In aqueous humor (figure 4-2b), the ketorolac concentration at 4 hours was significantly higher $(p=0.004)$ for the ocular coil group compared to the eye drop group $(2780 \pm 1485 \mathrm{ng} / \mathrm{mL}$ and $983 \pm 629 \mathrm{ng} / \mathrm{mL}$, respectively). At 24 hours, the ketorolac concentration of the ocular coil $(162 \pm 120 \mathrm{ng} / \mathrm{mL})$ was comparable to that of eye drops $(206 \pm 116 \mathrm{ng} / \mathrm{mL})$, and at day 4 , the concentration was significantly higher $(p=0.001)$ in the eye drop group $(299 \pm 205 \mathrm{ng} / \mathrm{mL}$ versus $10 \pm 11 \mathrm{ng} / \mathrm{mL})$. After day 4 , the concentration aqueous humor of the ocular coil group dropped below the detection limit whereas it could be measured in the eye drop group $(52 \pm 8 \mathrm{ng} / \mathrm{mL}$ and $94 \pm 74 \mathrm{ng} / \mathrm{mL}$ for day 7 and day 28 , respectively).

The ketorolac concentration in plasma (figure $4-2 c$ ) at 4 hours was ten times higher $(p=0.006)$ in the ocular coil group compared to the eye drop group $(148 \pm 128 \mathrm{ng} / \mathrm{mL}$ and $14 \pm 9 \mathrm{ng} / \mathrm{mL}$, respectively). At 24 hours, the plasma concentration was equal for both groups $(7 \pm 5 \mathrm{ng} / \mathrm{mL}$ and $7 \pm 3 \mathrm{ng} / \mathrm{mL}$, for the ocular coil and the eye drop group, respectively). After day 4 , the concentration in the ocular coil group dropped below the detection limit where the plasma concentration of the eye drop group was $16 \pm 12 \mathrm{ng} / \mathrm{mL}, 16 \pm 12 \mathrm{ng} / \mathrm{mL}$, and $12 \pm 9 \mathrm{ng} / \mathrm{mL}$ for days 4,7 , and 28 , respectively.
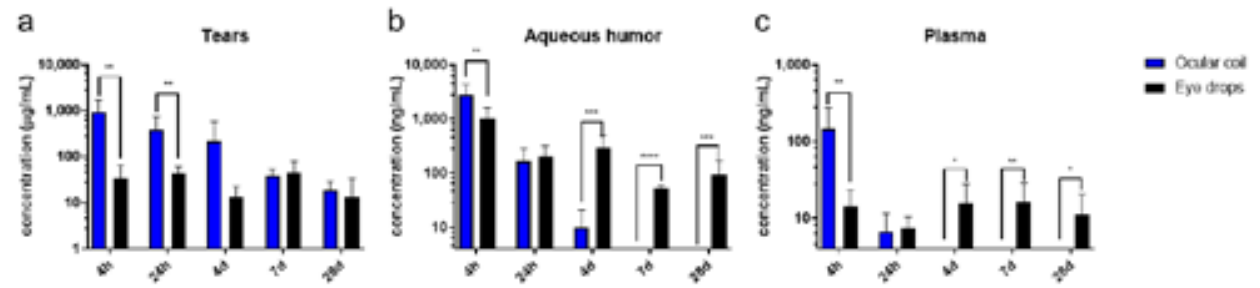

Figure 4-2. Pharmacokinetics of the ocular coil. Concentration ketorolac detected in (a) tears, (b) aqueous humor, and (c) plasma. $\mathrm{N}=9$ rabbits per group, data are plotted as mean $\pm \mathrm{SD}$. ${ }^{*} \mathrm{p}<0.05,{ }^{* *} \mathrm{p}<0.01$, ${ }^{* * *}$ $p<0.001$, and ${ }^{* * * *} p<0.0001$

\subsubsection{Efficacy of the ocular coil compared to eye drops and no treatment}

Efficacy was evaluated by measuring the total protein concentration and the concentration of cytokines in tears, aqueous humor, and plasma after inducing an ocular inflammation. The inflammation was treated using the ocular coil, eye drops, or left untreated. Figure 4-3 provides an overview of the total protein concentration in tears, aqueous humor, and plasma for the three animal groups. In tears (figure 43a), no large differences in the total protein concentration were observed within the treatment groups. At baseline, however, difference between the control group and 
eye drops $(p=0.031)$ was seen, and at day 14 , decrease of the total protein concentration was observed in the control group $(p=0.032)$.

In aqueous humor (figure 4-3b), the total protein concentration strongly increased at 4 hours from baseline in all animal groups. At 8 hours, the total protein concentration was only elevated in the control group $(p<0.0001)$ and was back to baseline in the ocular coil group and the eye drop group. At 24 hours, the total protein concentration was back at baseline level for all groups. Comparing the different groups, the total protein concentration in aqueous humor in the control group was higher compared to the ocular coil group at 4 hours $(p=0.025)$, and higher compared to both treatment groups at 8 hours $(p<0.0001)$.

In plasma (figure 4-3c), a horizontal trend without peaks was observed. The total protein concentration is only higher when compared to baseline in the eye drop group at 24 hours $(p=0.013)$.
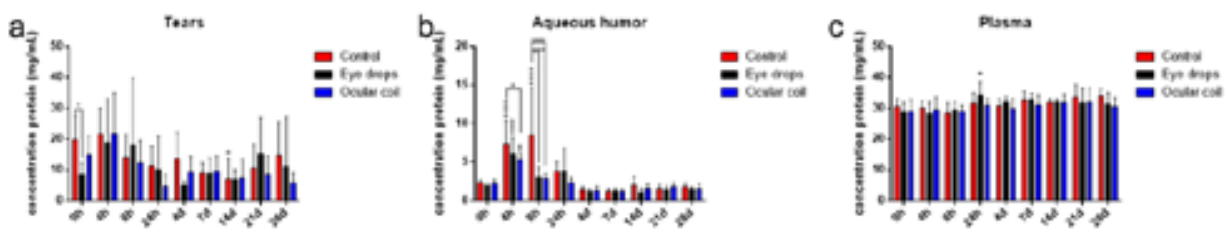

Figure 4-3. Total protein concentration in (a) tears, (b) aqueous humor, and (c) plasma. $\mathrm{N}=8$ rabbits per group, data are plotted as mean $\pm \mathrm{SD}$. '") Indicates significance compared to baseline condition, '*' indicates a difference between two groups.

The concentration of $\mathrm{PGE}_{2}$, an inflammatory mediator that is released immediately after inflammation, is depicted in figure 4-4. In tears (figure 4-4a), the concentration $\mathrm{PGE}_{2}$ at 4 hours was higher in the control group compared to the eye drop group $(p=0.002)$. At day 4 , the $\mathrm{PGE}_{2}$ concentration was higher in the control group compared to the eye drop group $(p<0.0001)$ and the ocular coil group $(p=0.007)$. At day 21 , the $\mathrm{PGE}_{2}$ concentration was higher in the control group compared to the eye drop group $(p=0.048)$. In the control group, the $\mathrm{PGE}_{2}$ concentration was increased as compared to baseline at days $4(p=0.040)$ and $21(p=0.002)$.

In aqueous humor (figure 4-4b) $\mathrm{PGE}_{2}$ concentrations increased significantly in the control group at $4(p<0.0001), 8(p<0.0001)$, and $24(p<0.0001)$ hours after induction of the inflammation. However, when treated with eye drops, a delayed increase of $\mathrm{PGE}_{2}$ was observed. Increase in $\mathrm{PGE}_{2}$ was observed at 24 hours $(p=0.0005)$, at day $4(p=0.033)$, and at day $7(p=0.049)$ in the eye drop group, whereas treatment with the ocular coil did not result in significantly increased changes of $P E_{2}$. The control group had higher $\mathrm{PGE}_{2}$ levels compared to the eye drop group and the ocular coil group at 4 hours $(p<0.0001$ and $p=0.0002$, respectively), 8 hours $(p<0.0001$ and $p<0.0001$, respectively), and 24 hours $(p=0.028$ and $p=0.006$, respectively). 
In plasma (figure 4-4c), the $\mathrm{PGE}_{2}$ concentration was undetectable in the majority of samples. The $\mathrm{PGE}_{2}$ concentration in the control group was increased at 8 hours when compared to both treatment groups, as well as compared to baseline. No further changes compared to baseline or within the different groups were observed in plasma.
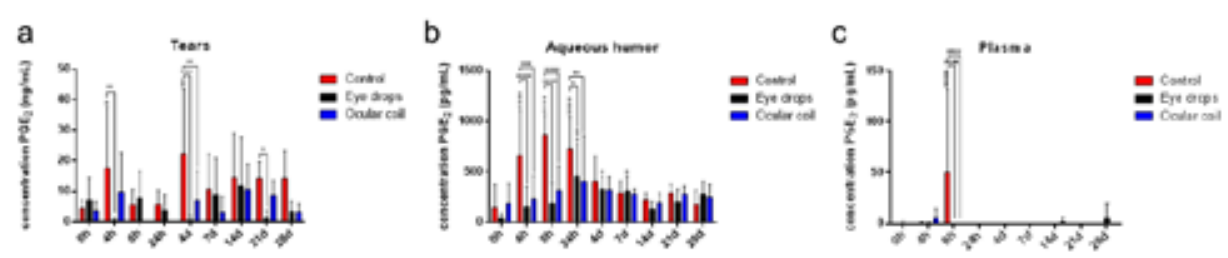

Figure 4-4. $\mathrm{PGE}_{2}$ concentration in (a) tears, (b) aqueous humor, and (c) plasma. $\mathrm{N}=8$ rabbits per group, data are plotted as mean \pm SD. '"' Indicates significance compared to baseline condition, '*' indicates a difference between two groups.

Figure 4-5 shows the concentration of TNFa, an inflammatory mediator related to the acute phase of inflammation, in tears, aqueous humor, and plasma. In tears (figure 4-5a), the TNFa concentration at 4 hours was higher in the eye drop group compared to the control group $(p=0.005)$ and the ocular coil group $(p=0.001)$. In tears, an increased TNF $\alpha$ concentration was observed in the control group at day 4 $(p=0.005)$ and at day $14(p=0.001)$ compared to baseline. In the eye drop group, an increase in the TNF $\alpha$ concentration was observed at 4 hours $(p=0.005)$ compared to baseline.

In aqueous humor (figure 4-5b), at day 4, the concentration of TNFa was higher in the eye drop group $(p=0.040)$ and the ocular coil group $(p=0.004)$ compared to the control group. The TNF $\alpha$ concentration as compared to baseline was also increased in the ocular coil group at day $4(p=0.017)$. In plasma (figure 4-5c), the eye drop group shows increased TNFa at 4 hours compared to the eye drop group $(p=0.028)$. Furthermore, the ocular coil has increased TNFa at day $28(P<0.0001)$ compared to baseline.

The IL-6 concentration is plotted in figure 4-6, IL-6 is also an important mediator for the acute phase of inflammation. In tears (figure 4-6a), all three groups show elevated IL- 6 concentrations at 4 hours $(p<0.0001)$. However, no difference between the groups was observed for the different time points.

In aqueous humor (figure 4-6b), the concentration of IL-6 is higher in the control group at 8 hours compared to the eye drop group $(p<0.0001)$ and the ocular coil group ( $p<0.0001)$, and is also higher at 24 hours compared to the ocular coil group $(p<0.0001)$. At 24 hours, the eye drop group also has a higher IL-6 concentration compared to the ocular coil group $(p=0.004)$. Compared to baseline, IL-6 is elevated 
in the control group at 8 hours $(p<0.0001)$ and for all three groups at 24 hours. No changes in IL-6 levels have been observed in plasma (figure 4-6c).

Figure 4-7 shows the IL-1 $\beta$ concentration in tears and plasma, IL-1 $\beta$ induces cyclooxygenase (COX) and is found to contribute to inflammatory pain. The concentration was below detection limit in aqueous humor. In tears (figure 4-7a), the IL-1 $\beta$ concentration is higher in the eye drop group compared to the ocular coil group $(p=0.0005)$ at 4 hours. Furthermore, increase in IL-1 $\beta$ is observed at 4 hours in the control group $(p=0.021)$ and in the eye drop group $(p=0.002)$.

In plasma (figure 4-7b), no differences between the groups were observed. However, the eye drop group shows an increased IL-1 $\beta$ concentration at day $14(p=0.011)$ compared to baseline.
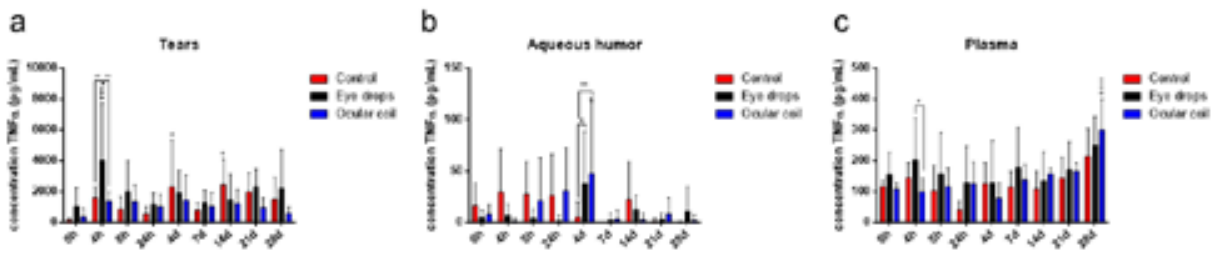

Figure 4-5. TNFa concentration in (a) tears, (b) aqueous humor, and (c) plasma. $\mathrm{N}=8$ rabbits per group, data are plotted as mean \pm SD. '+' Indicates significance compared to baseline condition, '*' indicates a difference between two groups.
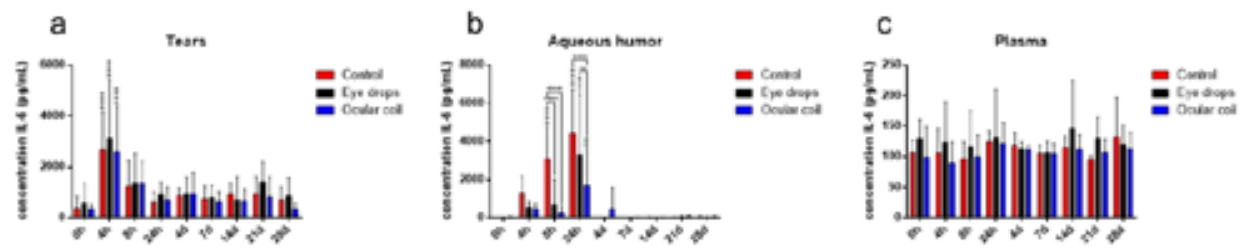

Figure 4-6. IL-6 concentration in (a) tears, (b) aqueous humor, and (c) plasma. $\mathrm{N}=8$ rabbits per group, data are plotted as mean \pm SD. '"+ Indicates significance compared to baseline condition, ' ${ }^{\prime \prime}$ indicates a difference between two groups.
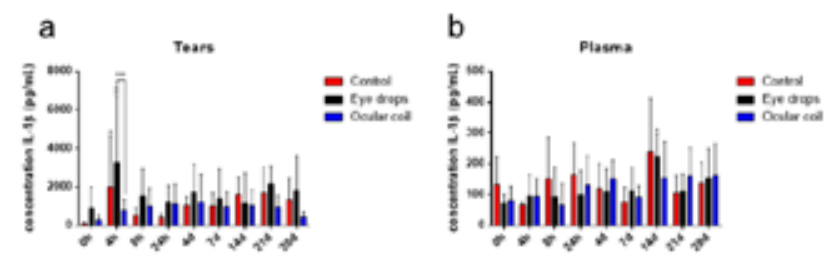

Figure 4-7. IL-1 $\beta$ concentration in (a) tears and (b) plasma. $\mathrm{N}=8$ rabbits per group, data are plotted as mean \pm SD. '"' Indicates significance compared to baseline condition, '*' indicates a difference between two groups. 


\subsection{Discussion}

The effectiveness of commonly prescribed eye drop therapies is often compromised due to low patient compliance.[2,4] Therefore, we developed a non-invasive drug delivery device called the ocular coil.[2,7-10] In this manuscript, we provided insights into the pharmacokinetics and efficacy of the ocular coil as an alternative to eye drops.

When comparing the pharmacokinetics of both delivery methods, higher ketorolac concentrations were found at 4 hours in tears, aqueous humor, as well as plasma in the ocular coil group as compared to the eye drop group. Afterwards, ketorolac concentrations in both tears and aqueous humor from the ocular coil firmly decrease (approximately 100-fold), while ketorolac concentrations for eye drops remain similar. We believe that this difference is due to a difference in penetration into the anterior chamber (as concentration is a driver for penetration) and due to the lack of additives in the ocular coil to enhance penetration.

The ocular coil releases a single high dose (burst) of ketorolac where after drug release gradually lowers.[11] Applications that would greatly benefit from this burst release of drugs are acute inflammatory events such as (cataract) surgery induced inflammation or corneal ulcers that currently need fortified antibiotic application at an hourly dosing regimen during the first two days. Current drug release kinetics make the ocular coil not favorable for chronic diseases. Our results show that the ocular coil and eye drops achieve peak concentrations in aqueous humor of $2779.7 \pm$ $1484.9 \mathrm{ng} / \mathrm{mL}$ and $983.4 \pm 629.7 \mathrm{ng} / \mathrm{mL}$, respectively, after 4 hours. Bucci et al. reported peak concentrations of ketorolac in aqueous humor from cataract patients prior to surgery of $688.87 \pm 749.6 \mathrm{ng} / \mathrm{mL}$.[17] We would however expect higher concentrations in their study because they administer four additional eye drops one hour prior to surgery and because they sample quickly afterwards, while we sample 4 hours later. Furthermore, since we need to stitch the ocular coil in the conjunctiva (and mock stitch the eye drop group); we expected that part of the administered ketorolac is used and thus less free ketorolac would be available.

In general, drug release via eye drops sharply peaks after each application and disappears quickly due to tearing and blinking.[18-21] In our experimental set-up, sampling always took place at the same time after eye drop application. Therefore, ketorolac levels were similar at different time points and the drug profile resembles a steady-state drug release instead of a peak pattern.

We tested the efficacy of the ocular coil after induction of inflammation by paracentesis. In the untreated control group we observed a three-fold increase in the total protein concentration in aqueous humor at 4 and 8 hours after paracentesis. In particular a strong and steep increase in $\mathrm{PGE}_{2}$ concentration was observed. Already 4 hours after paracentesis, $\mathrm{PGE}_{2}$ concentrations were five-fold higher compared to 
baseline. The highest PGE 2 concentrations were observed at 4, 8 and 24 hours and slowly went back to baseline at day 28 .

In the ocular coil and eye drops, $\mathrm{PGE}_{2}$ concentrations mildly increased (although not significantly different from baseline), whereas concentrations increased significantly in the control group. The largest treatment effects were observed at 4,8 and 24 hours after paracentesis. Interestingly, the effect was similar for the ocular coil as for eye drops. These results suggest that different drug release patterns (burst release followed by gradual drug release versus single peak drug dosing) can yield the same treatment effect.

Differences in $\mathrm{PGE}_{2}$ concentrations between untreated and treated groups were only observed during the first 24 hours. After 4 days, $\mathrm{PGE}_{2}$ concentrations were back to baseline in all treated groups. This result raises questions regarding the intended treatment duration, which is currently set at 28 days for eye drops. Would a burst release of ketorolac be enough to halt the inflammatory cascade, or is prolonged exposure to the drug needed to achieve the optimal effect? This resembles a recent innovation in the pharmacological treatment of cataract surgery, where NSAIDS are provided during the surgery as an additive in the intraocular irrigation fluid. The use of a combination of ketorolac and phenylephrine (Omidria, Omeros corp, Seattle, WA, US) was effective in the prevention of postoperative inflammation and in the reduction of cystoid macular edema following surgery.[22]

For the current study, we used a repeated sampling animal model. In this model, a trauma-induced acute ocular inflammatory response was provoked by drawing a large volume of aqueous humor (150-175 $\mu \mathrm{L}$ ) (paracentesis) [23] followed by frequent sampling of small volumes $(50 \mu \mathrm{L})$. The advantage of this model is that repetitive sampling within the same animal generates data at multiple (paired) time points. Thereby, limiting the total numbers of animals needed. A drawback of this model is that only limited volumes of tear fluid, aqueous humor and plasma were available at each time point. Therefore, only few biomarkers could be tested thereby excluding the possibility to run technical replicates.

The performance of the drug-loaded ocular coil should be further validated in a clinical study. The in vivo pharmacokinetics of tears and in aqueous humor can be evaluated in patients undergoing regular cataract surgery. $[24,25]$ This would clarify whether similar intraocular concentrations can be achieved as a comparison to ketorolac solutions added to the irrigation fluid during surgery (Omidria) and could be equally effective in preventing a postoperative inflammatory response.

\subsection{Conclusion}

In this study, we compared the pharmacokinetic profile and efficacy of the ocular coil with eye drops. The ocular coil showed a burst release during the first days where 
after drug release gradually lowered. Despite differences in their drug release pattern, we showed that both delivery methods are able to suppress an induced inflammation in a repetitive sampling model in New Zealand White rabbits. Applications of the ocular coil may be a promising alternative for eye drops in ocular diseases where a burst release can effectively prevent or treat ocular inflammation.

\subsection{References}

1. Urtti, A., Challenges and obstacles of ocular pharmacokinetics and drug delivery. Adv Drug Deliv Rev, 2006. 58(11): p. 1131-1135.

2. Bertens, C.J.F., Gijs, M., van den Biggelaar, F., and Nuijts, R., Topical drug delivery devices: A review. Exp Eye Res, 2018. 168: p. 149-160.

3. Eaton, A.M., Gordon, G.M., Konowal, A., Allen, A., Allen, M., Sgarlata, A., Gao, G., Wafapoor, H., and Avery, R.L., A novel eye drop application monitor to assess patient compliance with a prescribed regimen: a pilot study. Eye (Lond), 2015. 29(10): p. 1383-1391.

4. Olthoff, C.M., Hoevenaars, J.G., van den Borne, B.W., Webers, C.A., and Schouten, J.S., Prevalence and determinants of non-adherence to topical hypotensive treatment in Dutch glaucoma patients. Graefes Arch Clin Exp Ophthalmol, 2009. 247(2): p. 235-243.

5. Mohindroo, C., Ichhpujani, P., and Kumar, S., How 'Drug Aware' are our Glaucoma Patients? J Curr Glaucoma Pract, 2015. 9(2): p. 33-37.

6. Farkouh, A., Frigo, P., and Czejka, M., Systemic side effects of eye drops: a pharmacokinetic perspective. Clin Ophthalmol, 2016. 10: p. 2433-2441.

7. Pijls, R.T., Hanssen, H.H., Nuijts, R.M., Daube, G.W., and Koole, L.H., In vivo tolerance and kinetics of a novel ocular drug delivery device. J Control Release, 2006. 116(2): p. e47-49.

8. Pijls, R.T., Cruysberg, L.P., Nuijts, R.M., Dias, A.A., and Koole, L.H., Capacity and tolerance of a new device for ocular drug delivery. Int J Pharm, 2007. 341(1-2): p. 152-161.

9. Pijls, R.T., Sonderkamp, T., Daube, G.W., Krebber, R., Hanssen, H.H., Nuijts, R.M., and Koole, L.H., Studies on a new device for drug delivery to the eye. Eur J Pharm Biopharm, 2005. 59(2): p. 283-288.

10. Pijls, R.T., Hanssen, H.H., Nuijts, R.M., and Koole, L.H., Flexible coils with a drug-releasing hydrophilic coating: a new platform for controlled delivery of drugs to the eye? Biomed Mater Eng, 2004. 14(4): p. 383-393.

11. Bertens, C.J.F., Martino, C., van Osch, M.C., Lataster, A., Dias, A., van den Biggelaar, F., Tuinier, R., Nuijts, R., and Gijs, M., Design of the ocular coil, a new device for non-invasive drug delivery. Eur J Pharm Biopharm, 2020. 150: p. 120-130.

12. Unger, W.G., Cole, D.F., and Hammond, B., Disruption of the blood--aqueous barrier following paracentesis in the rabbit. Exp Eye Res, 1975. 20(3): p. 255-270.

13. Sharma, N.S., Acharya, S.K., Nair, A.P., Matalia, J., Shetty, R., Ghosh, A., and Sethu, S., Dopamine levels in human tear fluid. Indian J Ophthalmol, 2019. 67(1): p. 38-41.

14. US Pharmacopeia. USP Ketorolac tromethamine. 2018 [cited 2018 November 26]; Available from: http://www.pharmacopeia.cn/v29240/usp29nf24s0_m44006.html.

15. Bertens, C.J.F., Zhang, S., Erckens, R.J., van den Biggelaar, F., Berendschot, T., Webers, C.A.B., Nuijts, R., and Gijs, M., Confocal Raman spectroscopy: Evaluation of a non-invasive technique for the detection of topically applied ketorolac tromethamine in vitro and in vivo. Int $\mathrm{J}$ Pharm, 2019. 570: p. 118641.

16. Motulsky, H.J. and Brown, R.E., Detecting outliers when fitting data with nonlinear regression a new method based on robust nonlinear regression and the false discovery rate. BMC Bioinformatics, 2006. 7(1): p. 123.

17. Bucci, F.A., Jr. and Waterbury, L.D., A randomized comparison of to-aqueous penetration of ketorolac $0.45 \%$, bromfenac $0.09 \%$ and nepafenac $0.1 \%$ in cataract patients undergoing phacoemulsification. Curr Med Res Opin, 2011. 27(12): p. 2235-2239.

18. Gaudana, R., Ananthula, H.K., Parenky, A., and Mitra, A.K., Ocular drug delivery. AAPS J, 2010. 12(3): p. 348-360.

19. Hughes, P.M., Olejnik, O., Chang-Lin, J.E., and Wilson, C.G., Topical and systemic drug delivery to the posterior segments. Adv Drug Deliv Rev, 2005. 57(14): p. 2010-2032. 
20. Lee, S.B., Geroski, D.H., Prausnitz, M.R., and Edelhauser, H.F., Drug delivery through the sclera: effects of thickness, hydration, and sustained release systems. Exp Eye Res, 2004. 78(3): p. 599-607.

21. Urtti, A. and Salminen, L., Minimizing systemic absorption of topically administered ophthalmic drugs. Surv Ophthalmol, 1993. 37(6): p. 435-456.

22. Visco, D.M. and Bedi, R., Effect of intracameral phenylephrine $1.0 \%$-ketorolac $0.3 \%$ on postoperative cystoid macular edema, iritis, pain, and photophobia after cataract surgery. J Cataract Refract Surg, 2020. 46(6): p. 867-872.

23. Graff, G., Brady, M.T., Gamache, D.A., Spellman, J.M., and Yanni, J.M., Transient loss of prostaglandin synthetic capacity in rabbit iris-ciliary body following anterior chamber paracentesis. Ocul Immunol Inflamm, 1998. 6(4): p. 227-238.

24. Walters, T., Raizman, M., Ernest, P., Gayton, J., and Lehmann, R., In vivo pharmacokinetics and in vitro pharmacodynamics of nepafenac, amfenac, ketorolac, and bromfenac. J Cataract Refract Surg, 2007. 33(9): p. 1539-1545.

25. Bucci, F.A., Jr. and Waterbury, L.D., Aqueous prostaglandin E(2) of cataract patients at trough ketorolac and bromfenac levels after 2 days dosing. Adv Ther, 2009. 26(6): p. 645-650. 


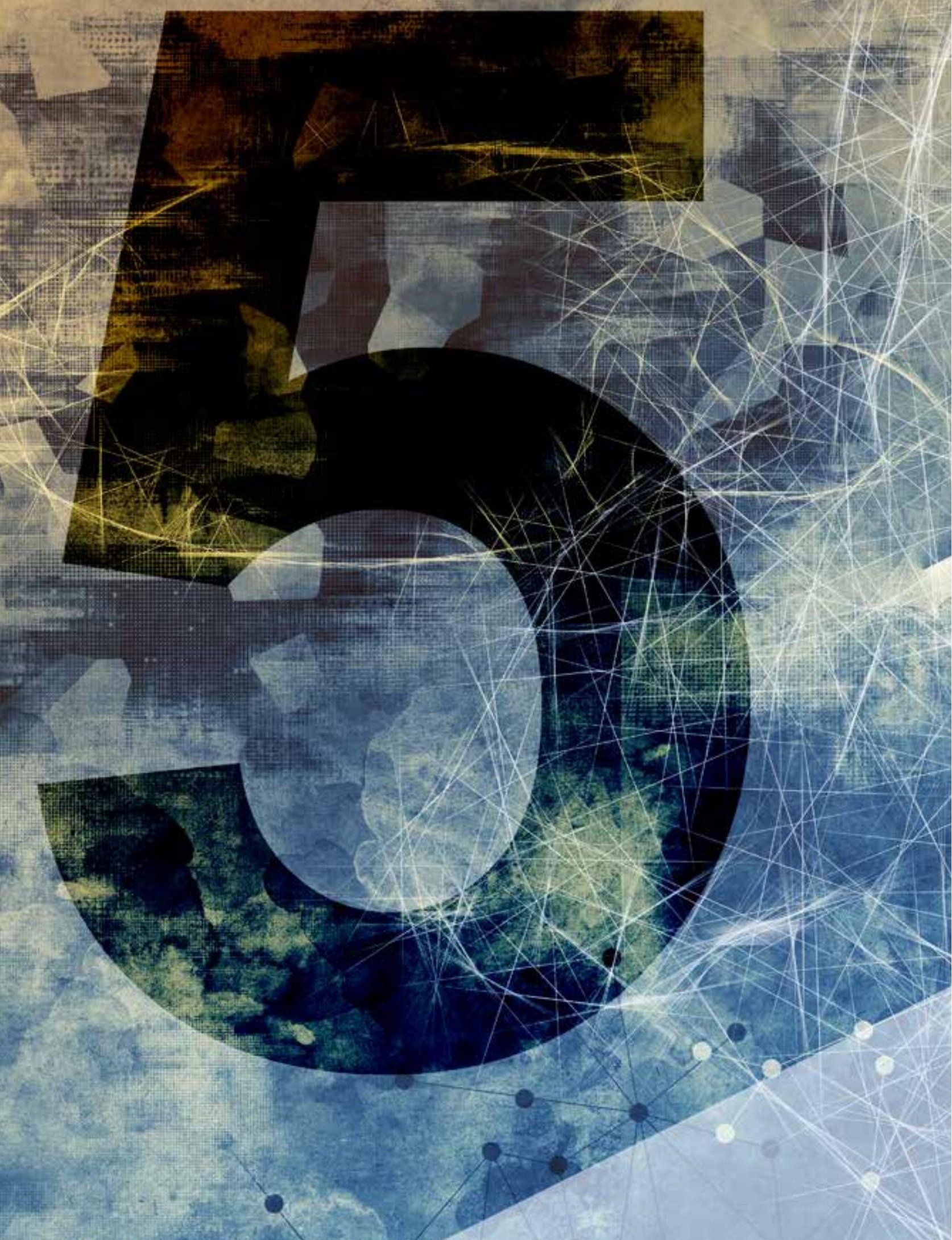


Chapter 5.

\section{Saffiy and comín of an}

innovative drug deliverry device in

\section{healthy subjests}

Christian J.F. Bertens, Suryan L. Dunker, Aylvin A. Dias, Frank J.H.M. van den Biggelaar, Rudy M.M.A. Nuijts, and Marlies Gijs

Translational Vision Science \& Technology. 2020 Dec 18;9(13):35.

DOI: $10.1167 /$ tvst.9.13.35 


\section{Abstract}

Purpose: The aim of the study was to investigate safety and comfort of two versions of a placebo-microsphere filled ocular coil (straight and curved) in healthy subjects. Methods: The study was a single-center intervention study. One ocular coil was placed in the inferior conjunctival fornix for the intended duration of 28 days. Fortytwo healthy adult subjects were included. At baseline, 30 minutes, 8 hours, 24 hours, 48 hours, 7 days, 14 days, 21 days, and 28 days after insertion, examinations were performed including slit lamp evaluation to score ocular redness, intraocular pressure measurement, visual acuity, tear secretion test and questionnaires.

Results: The straight and curved ocular coils had a median retention time of 5 days and 12 days, respectively. After 48 hours, $57 \%$ and $81 \%$ subjects retained the straight and curved ocular coil, respectively. Four (19\%) subjects with the straight coil and six $(29 \%)$ with the curved coil completed the entire study period. Minor changes in ocular hyperemia were observed in both groups. On day 7 , the straight coil was more comfortable than the curved coil with a visual analogue scale (VAS) score of $77 \pm 21$ compared to $94 \pm 11(P=0.028)$, respectively. No other ocular adverse events were observed.

Conclusions: Comfort and safety of the straight and curved ocular coil are high. Because the retention time is too short for long-term sustained drug release, the use in the perioperative or immediate postoperative period could prove to be more valuable.

Translational Relevance: The ocular coil is a non-invasive, comfortable and safe short term drug delivery device.
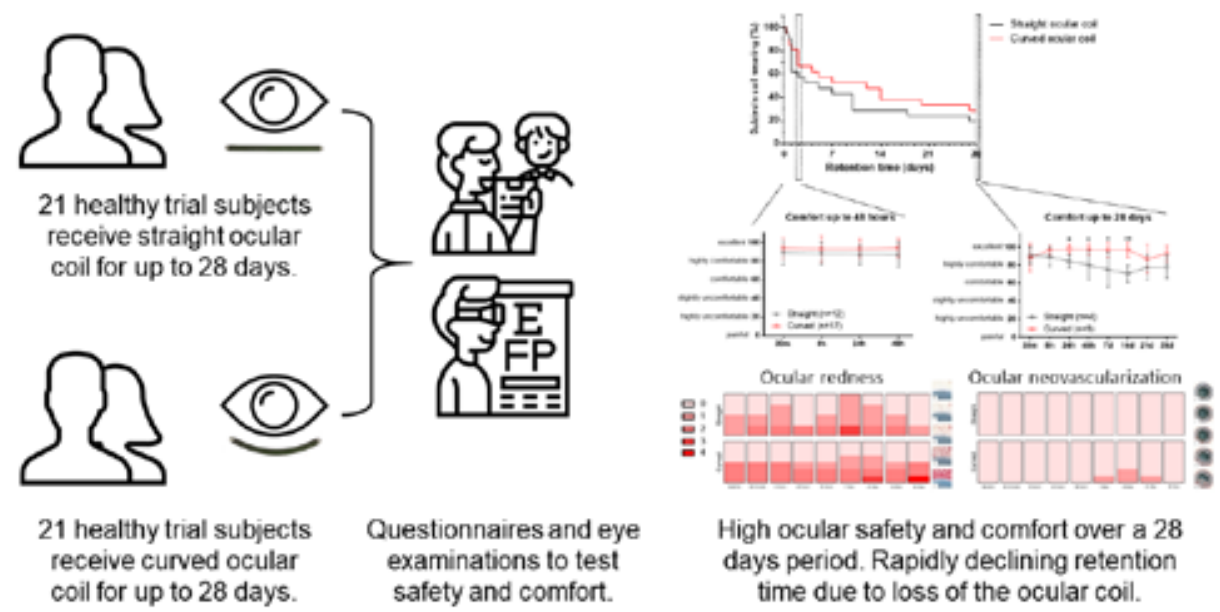

High ocular safety and comfort over a 28 days period. Rapidly declining retention time due to loss of the ocular coil. 


\subsection{Introduction}

Cataract surgery is one of the most performed surgeries in Western society.[1] To prevent postoperative complications, patients are treated with anti-inflammatory drugs for a period up to 28 days.[1-3] Postoperative drugs are mainly administered topical, via eye drops [4] because of their low costs and ease of use. However, the use of eye drops has several drawbacks. Besides systemic side effects [5] and local toxicity due to preservatives $[6,7]$, the main disadvantages of eye drops include low bioavailability [8-10] and poor patient compliance.[11-13] In order to address these problems, our group developed an ocular drug delivery device, the ocular coil. It is designed to rest in the inferior conjunctival fornix (figure 5-1a) in a non-invasive way and can be worn for a specific period of time. The benefits of a non-invasive drug delivery system are that it removes the burden of daily administrating topical drugs and, thereby, increases patient compliance.[14-17] The ocular coil is made from a coiled and coated stainless steel wire that is closed at both ends with a dome-shaped UV-curable acrylate urethane cap (figure 5-1b). The inner lumen of the ocular coil can be filled with a drug-eluting matrix for slow and sustained drug release.[18] For example, we developed ketorolac entrapped poly(methyl methacrylate) (PMMA) microspheres and inserted those into the inner lumen of the ocular coil. Release of ketorolac from the ocular coil occurred via diffusion from the microspheres. In an in vitro lacrimal system, a high dose of ketorolac was released (approximately $50 \%$ of the total loading) during the first 3 days, followed by sustained release until day 28.[18] Pilot studies showed that the ocular coil loaded with an atropine-releasing coating is able to achieve mydriasis [14], and that the ocular coil is safe and comfortable to wear for 2 hours.[17] The aim of the current clinical trial was to evaluate the safety and comfort of a straight and a curved ocular coil for an intended period of 28 days. In this study, we used an ocular coil that was filled with placebomicrospheres (figure 5-1d). Two versions of the ocular coil were evaluated. Initially, a straight ocular coil was designed to bend during wearing (figure 5-1b), followed by a curved ocular coil that was produced with an inherent curvature according to the outer circumference of the eye (figure 5-1c).

\subsection{Materials and Methods}

\subsubsection{Study design}

The study was designed as a unilateral randomized single-center intervention study. The study protocol was approved by the local ethics committee and the national authorities (number: NL57050.068.16/METC161042). The study procedures were performed in accordance with the tenets of the Declaration of Helsinki. The study 
was registered with the US National Institutes of Health Clinical Trials (ClinicalTrials.gov Identifier: NCT03488017).
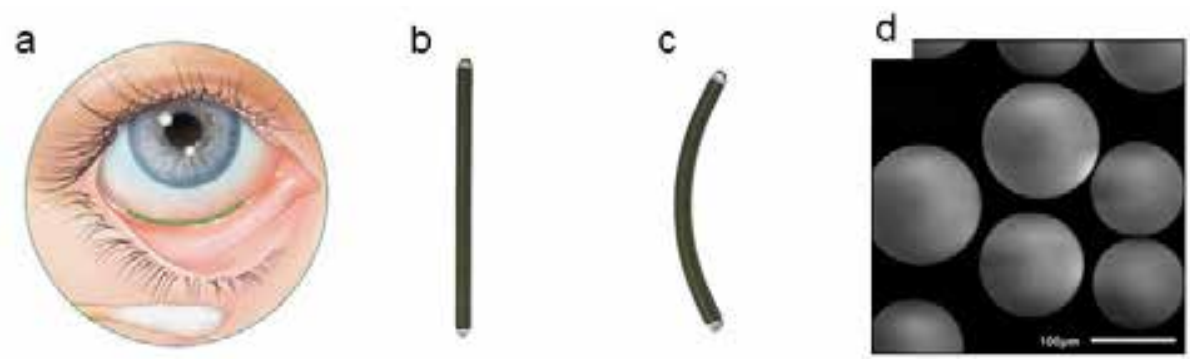

Figure 5-1. (a) Location of the ocular coil in the inferior conjunctival fornix. (b) Photograph of a straight ocular coil and (c) a curved ocular coil. (d) Scanning Electron Microscopic (SEM) photograph of the microsphere filling of the ocular coil (SEl, $1 \mathrm{kV}, 220 \times$ magnification).

\subsubsection{Study population}

Initially, the study was designed as a proof-of-concept study for the straight ocular coil in 40 subjects. However, after observing high occurrence of loss of the ocular straight coil in 21 subjects, inclusion was stopped and the ocular coil was redesigned to a curved ocular coil. After obtaining additional ethical approval, another 21 subjects were included to evaluate the curved ocular coil.

Subjects were included at the University Eye Clinic Maastricht, Maastricht, the Netherlands. From June 2018 until July 2019, 42 healthy adult subjects (between the age of 18 and 75 years) were included for the study with the ocular coil. All subjects gave written informed consent before inclusion. One eye per subject was included and one ocular coil was administered per eye. Exclusion criteria were any history of eye disease, allergies and hypersensitivity of the eye, current use of eye drops, contact lens use, inability to speak or write Dutch, Asian ethnicity (due extra subcutaneous fat in the eyelids), pregnant or breastfeeding women, or women with the intention of becoming pregnant during the study.

\subsubsection{Study procedures}

Before subjects were invited for a screening visit, the inclusion and exclusion criteria were checked. Subjects eligible for participation signed informed consent and underwent a screening session. The screening included an extensive ophthalmologic examination, slit lamp evaluation and photography, intraocular pressure (IOP) measurement (Icare-PRO, Vantaa, FI), corneal topography (Pentacam HR, Oculus, Irvine, CA, US), Schirmer's tear production test II (TEARstrips, Contacare Ophthalmics \& Diagnostics, Gujarat, IN), and visual acuity (best-corrected and uncorrected) using the Early Treatment Diabetic Retinopathy Study (ETDRS) chart.[19] Moreover, subjects were asked to complete the National 
Eye Institute Visual Function Questionnaire-25 (VFQ-25, version 2000) [20] with six detailed questions about ocular discomfort (table 5-S1).

At all visits, slit lamp evaluation (conjunctival and limbal hyperemia, corneal neovascularization, and edema) was performed using a Haag-Streit BX900 slit lamp bio-microscope (Haag Streit AG, Bern, $\mathrm{CH}$ ) to score according to the Efron grading scale (ranging from $0=$ normal, to $4=$ severe) [21]. Furthermore, conjunctival and corneal punctate staining was scored according to Bron et al. [22], and anterior chamber cells and flare were scored using the Standardization of Uveitis Nomenclature (SUN) classification.[23] Corneas were stained to visualize epithelial damage using fluorescein (Bausch \& Lomb, Rochester, NY, US). Additionally, subjects were asked to complete a customized questionnaire (table 5-S2).[16] Comfort of the ocular coil was scored using the visual analogue scale (VAS, 0-100, table 5-S2).

Using a computer algorithm, one eye of each subjects was randomly selected for insertion of the ocular coil. A trained physician inserted the ocular coil in the inferior conjunctival fornix using a Malosa Medical lens folding forceps triangular (\#1131, Malosa Limited, Elland, UK) after topical sedation with Oxybuprocaine hydrochloride (MINIMS, Bausch \& Lomb Pharma, Brussels, BE). The lower eyelid was retracted using the thumb and index finger and the ocular coil was gently placed into the fornix (figure 5-2).

After insertion of the ocular coil, eyes of subjects were evaluated at 30 minutes, 8 hours, 24 hours, 48 hours, 7 days, 14 days, 21 days, and 28 days, and after the ocular coil was removed. When intermediate loss of the ocular coil occurred (and was noticed by the subject), the subject was invited for a close-out visit. When loss of the ocular coil was noticed during one of the follow-up visits (unnoticed by the subject), data from the previous visit was used as the last day that the ocular coil was worn. After inclusion of the $13^{\text {th }}$ subject, a medical eye shield (Dispo Medical $\mathrm{BV}$, Hattemerbroek, NL) was introduced to prevent unintentional eye rubbing and dislodging of the ocular coil during sleep.
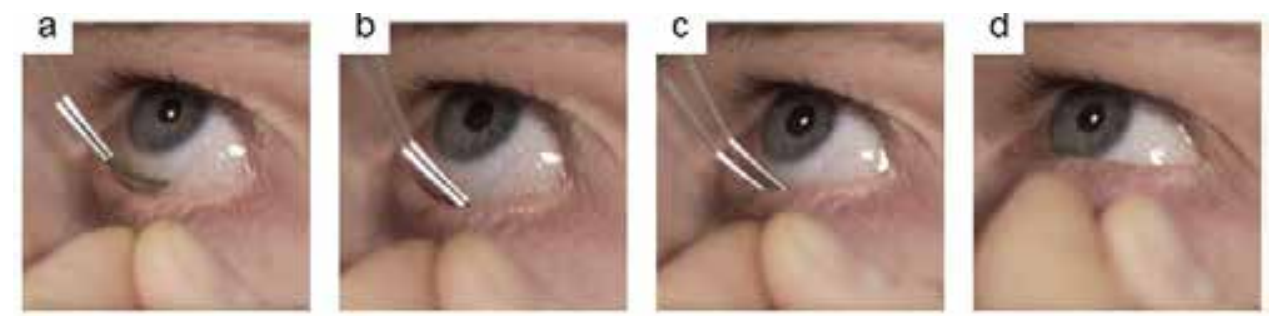

Figure 5-2. Insertion of the ocular coil. A pocket is made using index finger and thumb (a) and the ocular coil is diagonally inserted into the fornix (b). The ocular coil was gently released into the fornix (c), after insertion, the lower eyelid is released (d) and after a blink, the ocular coil lies in place. 


\subsubsection{Outcome parameters}

The primary outcome parameters of the study were conjunctival and limbal hyperemia, corneal defects, and ocular inflammation as determinants of the safety of the ocular oil. Secondary objectives were ocular coil retention time, subject comfort (tolerance) and pain, and incidence of adverse effects and complications (punctate keratitis, conjunctivitis, conjunctival or corneal erosion, and corneal ulceration).

\subsubsection{Statistical analysis}

In this study, two shapes of the ocular coil were tested. Originally, 40 subjects were planned to evaluate the straight ocular coil. However, due to low retention, a redesign of the shape of the ocular coil was needed. This resulted in a lower number of subjects and insufficient statistical power to evaluate safety parameters of the ocular coil.

Difference in age between the study populations for the straight and curved ocular coil was tested using an unpaired t-test. Difference in gender and study eye between the two study arms was tested with the chi-square test. Retention time of the straight and curved ocular coils was compared using the Mantel-Cox log-rank test. Mean and median of the retention time were tested using an unpaired t-test and a Mann Whitney rank sum test, respectively.

Due to the high number of missing data (due to variable loss of the coil), three complete case analyses were performed (i.e. for subjects who had a retention time up to 48 hours, up to 7 days, and for subjects who completed the entire study of 28 days).

Comparison of comfort of both ocular coils was done using multiple t-tests with a Bonferroni correction for multiple testing.

Tear migration length was compared using a paired t-test.

\subsection{Results}

\subsubsection{Study population}

Figure 5-3 shows a flow diagram of the number of subjects who were approached, screened, included, randomized, and analyzed in the study. In total, 106 information packages were sent to persons that showed interest to participate. In total, $47(45 \%)$ of the interested persons were invited for screening. During screening, 5 subjects (21\%) were found not eligible for participation due to their ocular condition, and 42 healthy subjects were included in the study.

Demographics of the subjects are shown in table 5-1. In the straight ocular coil arm, 12 subjects $(57 \%)$ and 9 subjects $(43 \%)$ received the ocular coil in their right and left eyes, respectively. In the curved ocular coil arm of the study, 10 subjects $(48 \%)$ 
received an ocular coil in the right eye and $11(52 \%)$ received an ocular coil in the left eye. The percentage of female subjects' study who received the curved versus the straight ocular coil was $67 \%$ and $52 \%$, respectively.

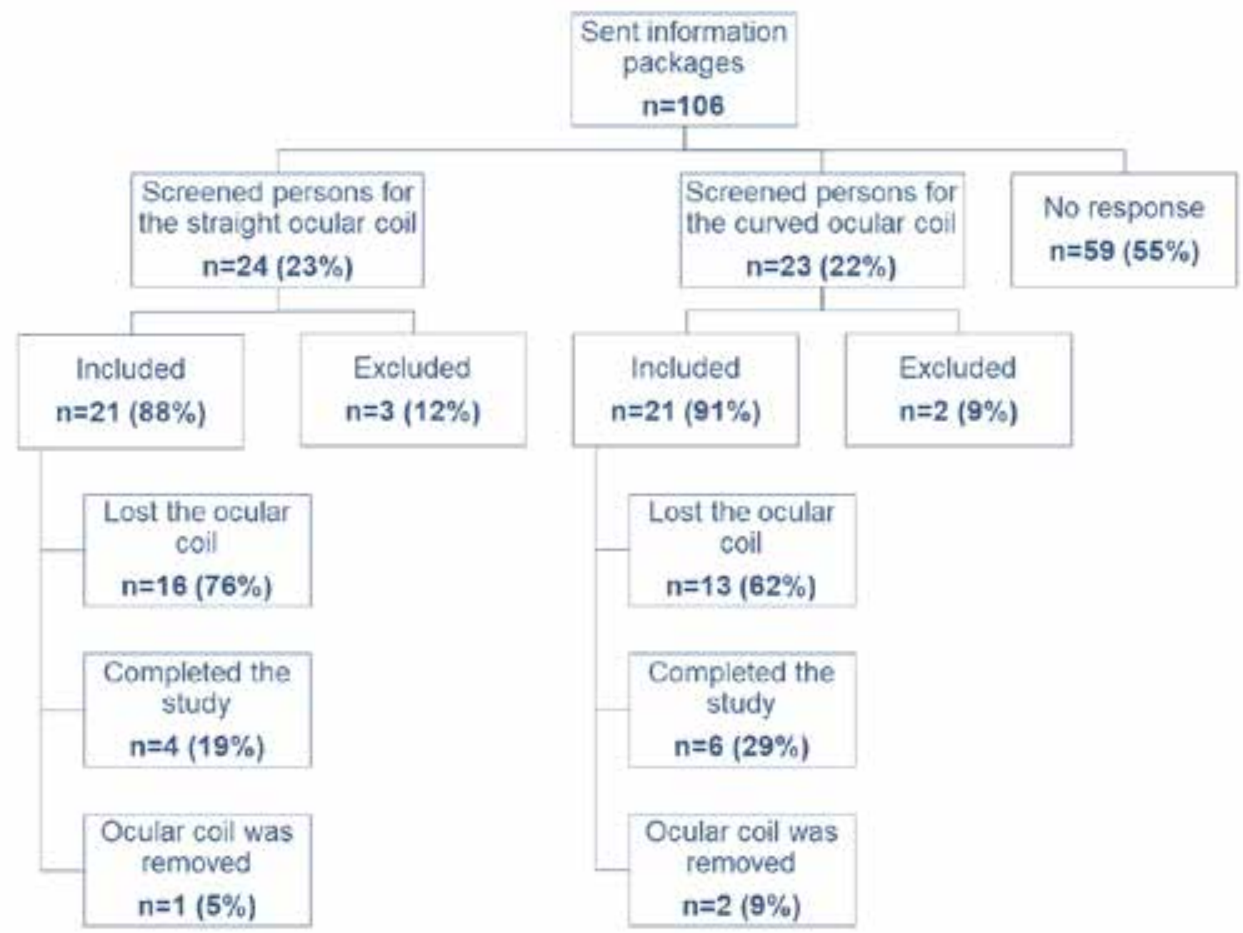

Figure 5-3. Flow diagram showing the number of subjects who were screened, included, randomized, and analyzed for both studies.

Table 5-1. Subject characteristics for both versions of the ocular coil.

\begin{tabular}{llll}
\hline Parameter & Straight coil & Curved coil & P value \\
\hline Mean age \pm SD (years) & $53 \pm 19$ & $55 \pm 19$ & 0.83 \\
\hline Range age (min-max) (years) & $22-74$ & $21-74$ & N.A. \\
\hline Gender ratio, male (\%) / female (\%) & $\delta 10(48 \%) /$ & $\uparrow 7(33 \%) /$ & 0.35 \\
& $+11(52 \%)$ & $+14(67 \%)$ & \\
Study eye OD (\%) / OS (\%) & $12(57 \%) /$ & $10(48 \%) /$ & 0.54 \\
& $9(43 \%)$ & $11(52 \%)$ & \\
Difference in age is tested using unpaired students t-test, gender difference and study eye is tested using \\
Chi-square test. N.A., not applicable.
\end{tabular}

\subsubsection{Retention}

Retention is defined as the period of time a subject was wearing the ocular coil. Retention of the straight and curved ocular coil is depicted in figure 5-4. For the 
straight ocular coil, 2 out of 21 subjects lost the ocular coil within one day. After 48 hours and 1 week, $12(57 \%)$ and $10(47 \%)$ of 21 subjects were still wearing the straight ocular coil, respectively. Four (19\%) subjects succeeded to wear the straight ocular coil for the full study period of 28 days.

For the curved ocular coil, the retention is also plotted in figure 5-4. Three subjects lost the ocular coil within 1 day. After 48 hours, $17(81 \%)$ subjects were wearing the curved ocular coil, after 1 week, 12 (57\%) subjects were still wearing the ocular coil. Six $(29 \%)$ subjects have worn the curved ocular coil for the full study period of 28 days.

No statistical difference $(P=0.38)$ in retention time between the straight and the curved ocular coil was observed. For the curved coil as compared to the straight coil, mean retention time slightly increased from $10 \pm 11$ days to $13 \pm 12$ days $(P=0.36)$, and median retention time increased from 5 days to 12 days $(P=0.35)$, respectively (figure 5-4).

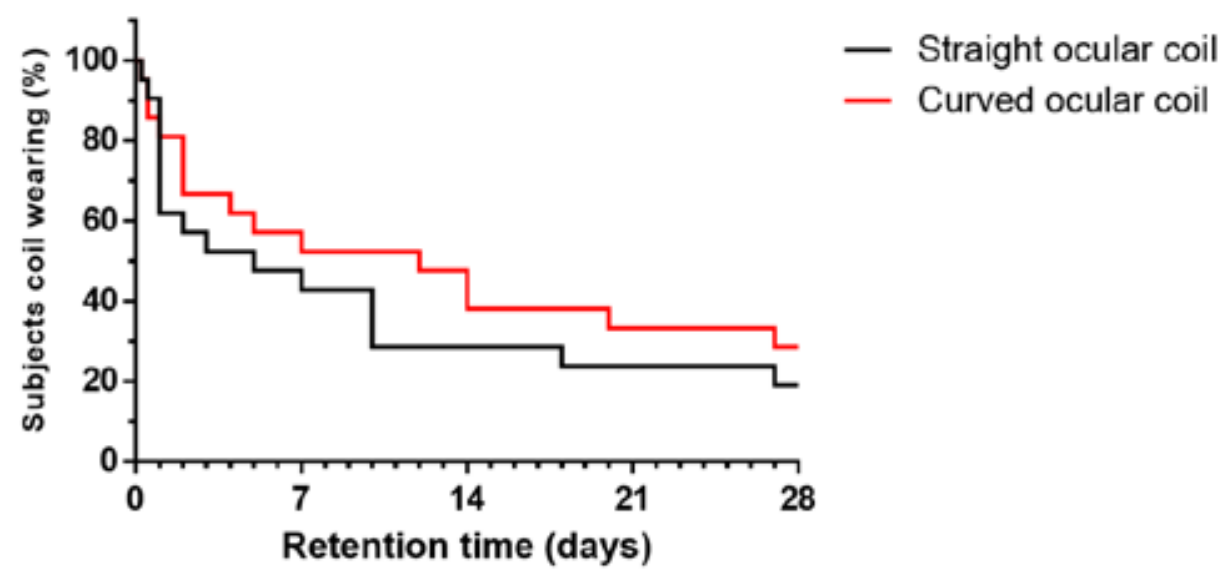

\begin{tabular}{|l|c|c|}
\hline & Mean \pm SD (days) & Median (days) \\
\hline Straight ocular coil & $10.0 \pm 11.1$ & 5 \\
\hline Curved ocular coil & $13.3 \pm 11.7$ & 12 \\
\hline P value & 0.36 & 0.35 \\
\hline
\end{tabular}

Figure 5-4. Retention of the straight and curved ocular coil during the study period of 28 days. $P=0.38$ using the Mantel-Cox test. Testing difference between the means using students t-test $P=0.36$ and difference between median using Mann Whitney rank test $\mathrm{P}=0.35$.

Reasons for loss of the curved and straight ocular coils are listed in table 5-2. Eye rubbing was the major cause of loss of the ocular coil in the straight ocular coil group, whereas a majority of subjects in the curved ocular coil group where not aware of loss. One subject removed the ocular coil from the eye after it protruded nasally. In three cases, the ocular coil was removed upon request. In the first case, the ocular 
coil was removed on the day of insertion because the subject complained about pain after getting a twig (from a tree) in his/her eye. Ocular examination revealed a corneal erosion (figure 5-S1). In the second case, the ocular coil was removed after 14 days due to foreign body sensations, and in a third case, the ocular coil was removed because it migrated to the upper eyelid, causing irritation (figure 5-S2a).

Table 5-2. Reasons for loss of the ocular coil

\begin{tabular}{l|cc}
\hline Reasons for loss of the ocular coil & $\begin{array}{c}\text { Straight coil } \\
(n=17 / 21)\end{array}$ & $\begin{array}{c}\text { Curved coil } \\
(n=15 / 21)\end{array}$ \\
\hline Eye rubbing / manipulating the eye & 7 & 1 \\
During sleep (without eye shield) & 3 & N.A. \\
During sleep (with eye shield) & 0 & 3 \\
Changing clothes & 1 & 1 \\
Checking whether the ocular coil was still in the & 2 & - \\
fornix & & - \\
Removed the coil because of nasal protrusion & 1 & 9 \\
Unknown reason & 1 & 1 \\
Removed upon request & 2 & \\
N.A., not applicable & &
\end{tabular}

\subsubsection{Safety}

Conjunctival hyperemia is plotted in figure 5-5. The mean hyperemia score for subjects wearing the straight ocular coil and the curved ocular coil for the first 48 hours was $0.75 \pm 0.75$ and $0.71 \pm 0.99$, for the 7 days period was $0.68 \pm 0.75$ and $0.68 \pm 0.85$, and for the 28 day period was $0.78 \pm 0.83$ and $1.00 \pm 1.05$, respectively. For the first 48 hours, conjunctival hyperemia was similar for both ocular coils. At 7 days, conjunctival hyperemia slightly lowered for both ocular coils, however hyperemia of the curved ocular coil seems to show less fluctuations compared to the straight ocular coil. One subject wearing a straight ocular coil had a conjunctival hyperemia score of "3" (moderate) at day 7 for unknown reasons that did not lead to other complaints. Two other subjects wearing a curved ocular coil presented with increased conjunctival hyperemia on day 14 and day 28, respectively. The latter was related to a hyposphagma due to eye rubbing (figure 5-S3).

Only minor changes were observed when scoring limbal hyperemia (figure 5-6). This also applied to corneal neovascularization (figure 5-7). A slight increase in neovascularization was observed in the curved ocular coil group but disappeared at day 28.

No signs of anterior chamber inflammation were noticed with a maximum of one cell observed (SUN guidelines [23]) in the anterior chamber, and no presence of flare in any subject during the study (data not shown). Visual acuity, IOP, and corneal 
topography of all subjects did not differ at any visit compared to baseline (data not shown).

\subsubsection{Comfort}

Comfort was scored at each follow-up visit through a questionnaire and a VAS score. figure 5-8 shows comfort of both ocular coils as complete case analysis for the first 48 hours (figure 5-8a), up to day 7 (figure 5-8b) and day 28 (figure 5-8c), whereas figure 5-8d shows comfort of all subjects. Overall, both ocular coils were found comfortable to wear during the first 48 hours (figure 5-8a). Although both coils were considered highly comfortable to excellent, the curved ocular coil was more comfortable at day 7 compared to the straight ocular coil (VAS of $77 \pm 21$ compared to $94 \pm 11, P=0.028$, respectively, figure $5-8 b$ ). Furthermore, the curved ocular coil showed less fluctuations in comfort between 30 minutes and 7 days.

For subjects that completed the study, the curved ocular coil was more comfortable after 24 hours (VAS score of $84 \pm 7$ vs. $98 \pm 6 ; P=0.011$ ), 48 hours (VAS score of 80 \pm 16 vs. $97 \pm 7 ; P=0.044$ ), 7 days (VAS score of $75 \pm 19$ vs. $97 \pm 8 ; P=0.034$ ), and 14 days (VAS score of $78 \pm 17$ vs. $97 \pm 8$; $P=0.001$, figure $5-8 c$ ) as compared to the straight coil. The curved coil also provided less fluctuation in comfort over a period of 28 days, compared to the straight ocular coil. No statistical difference in comfort between 30 minutes and 28 days was observed. Comparing all subjects, significant difference in comfort between the straight and curved ocular coil is only found on day 7 (VAS score of $77 \pm 21$ vs. $94 \pm 10$, $p=0.0019$, figure $5-8 d$ ).

During the follow-up moments the subjects were asked several questions (table 5S2), such as whether they feel the ocular coil (figure 5-9) and whether it is uncomfortable to have the ocular coil in their fornix (figure 5-10). Overall, more persons noted the ocular coil in their eye in the straight ocular coil group compared to the curved ocular coil group. The presence of the straight ocular coil was considered slightly more uncomfortable than the curved ocular coil. At day 14 , one subject found the ocular coil uncomfortable to wear, therefore, the ocular coil was removed upon request, due to foreign body sensations. 


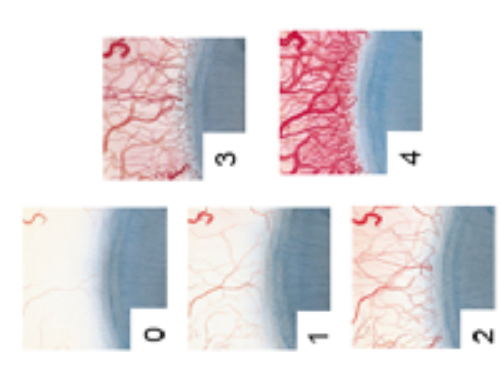

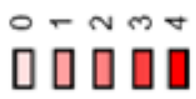

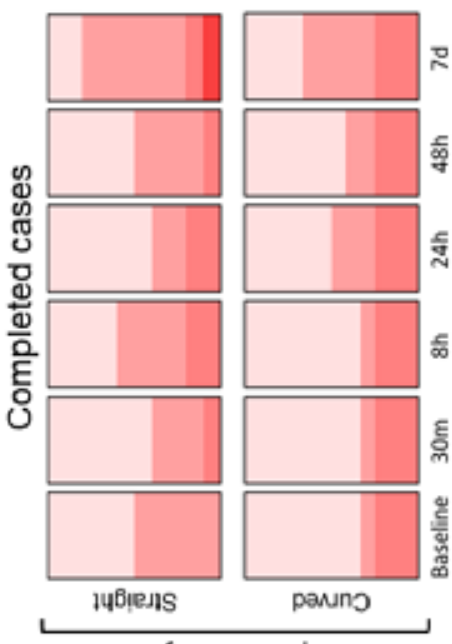

sKep $\angle$ ol dn

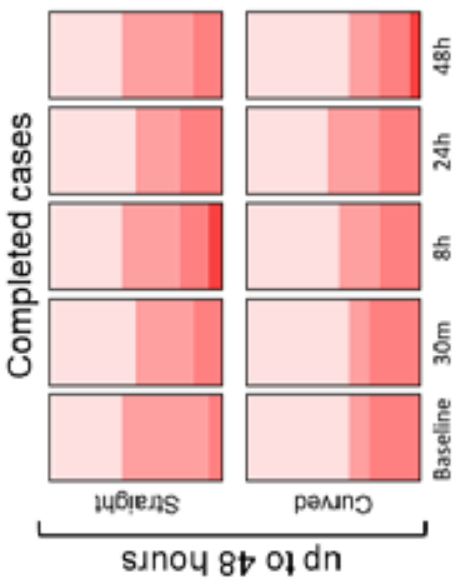

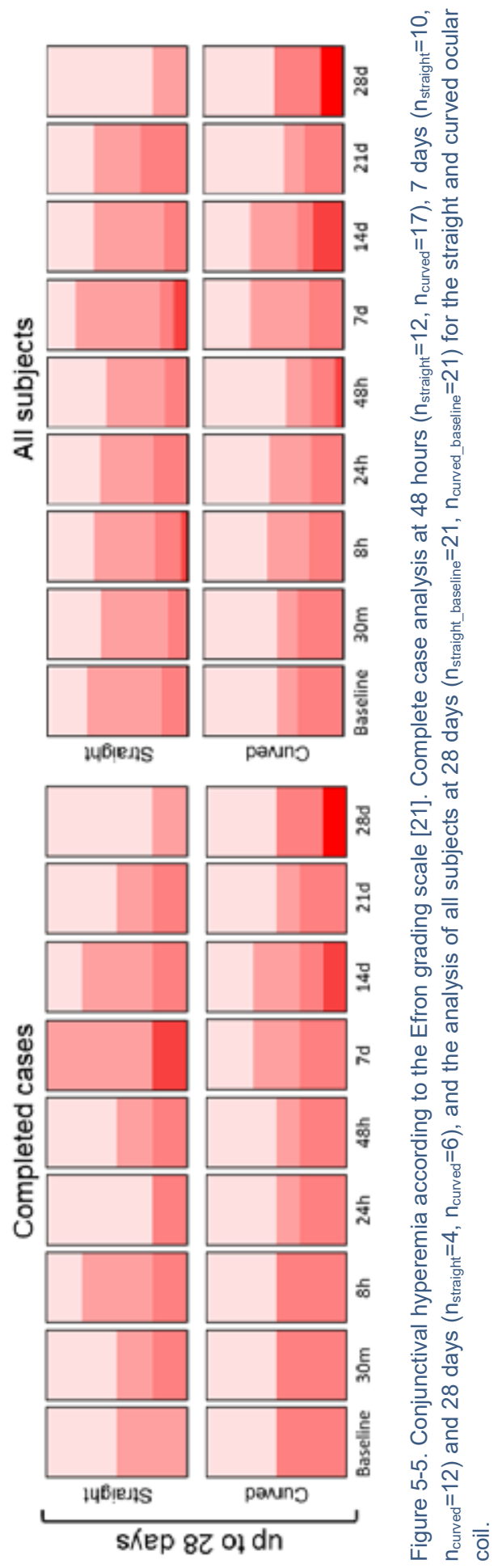



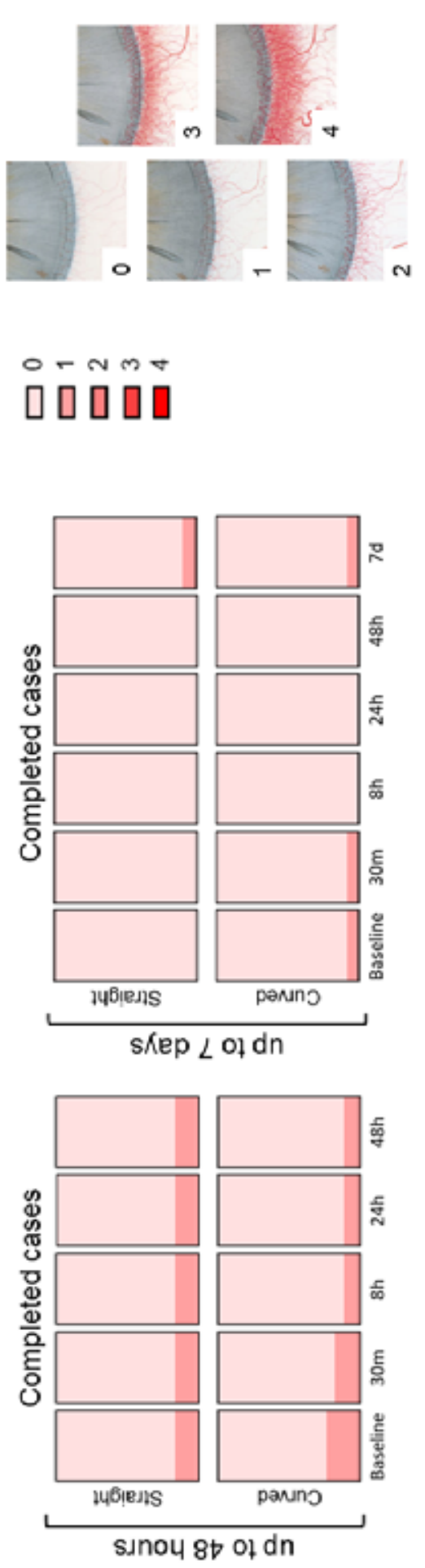

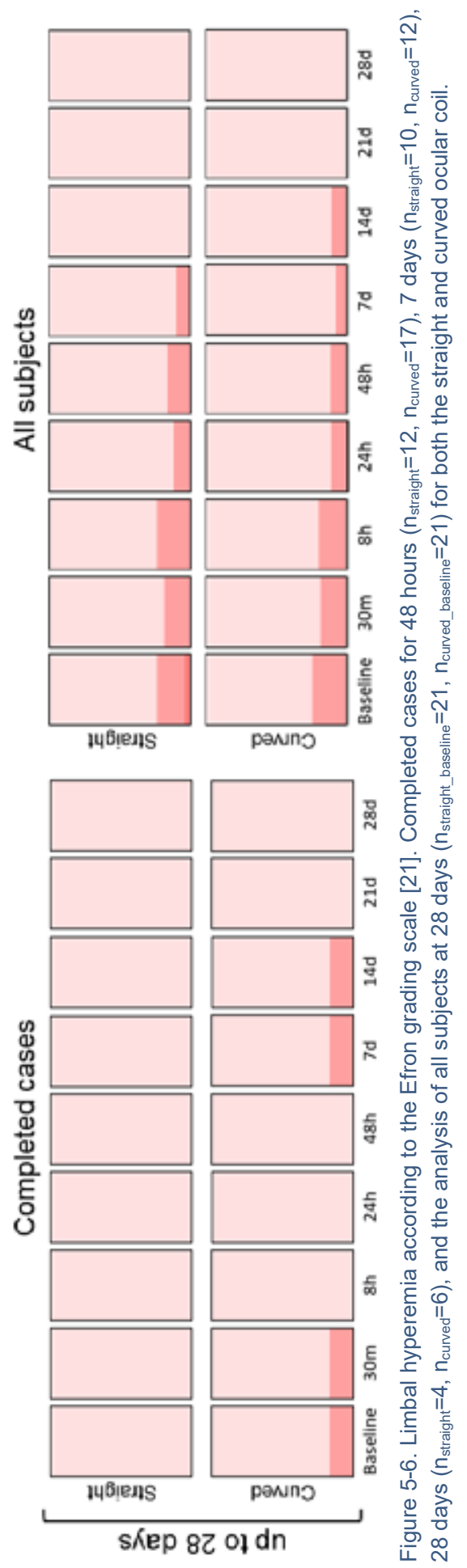



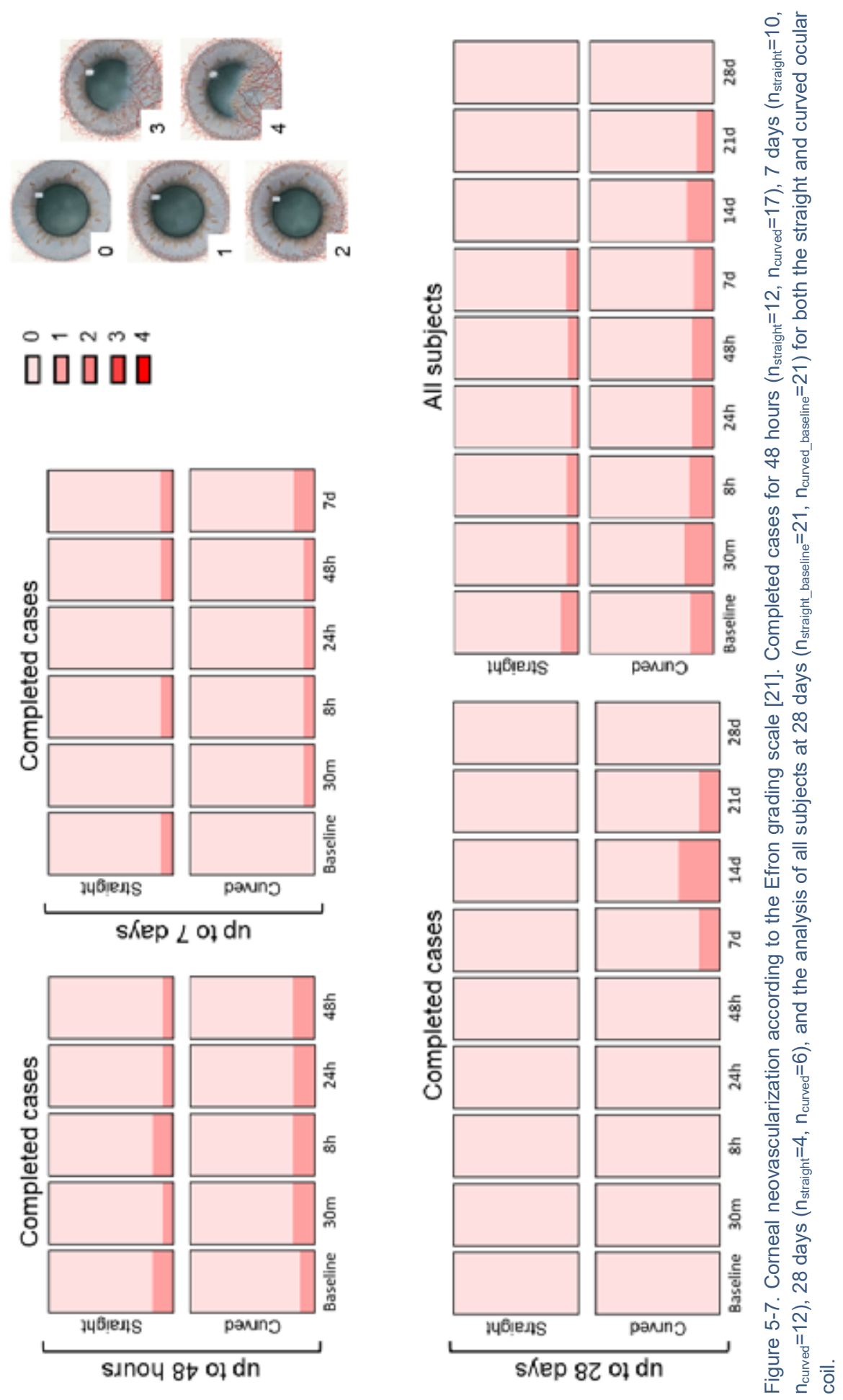


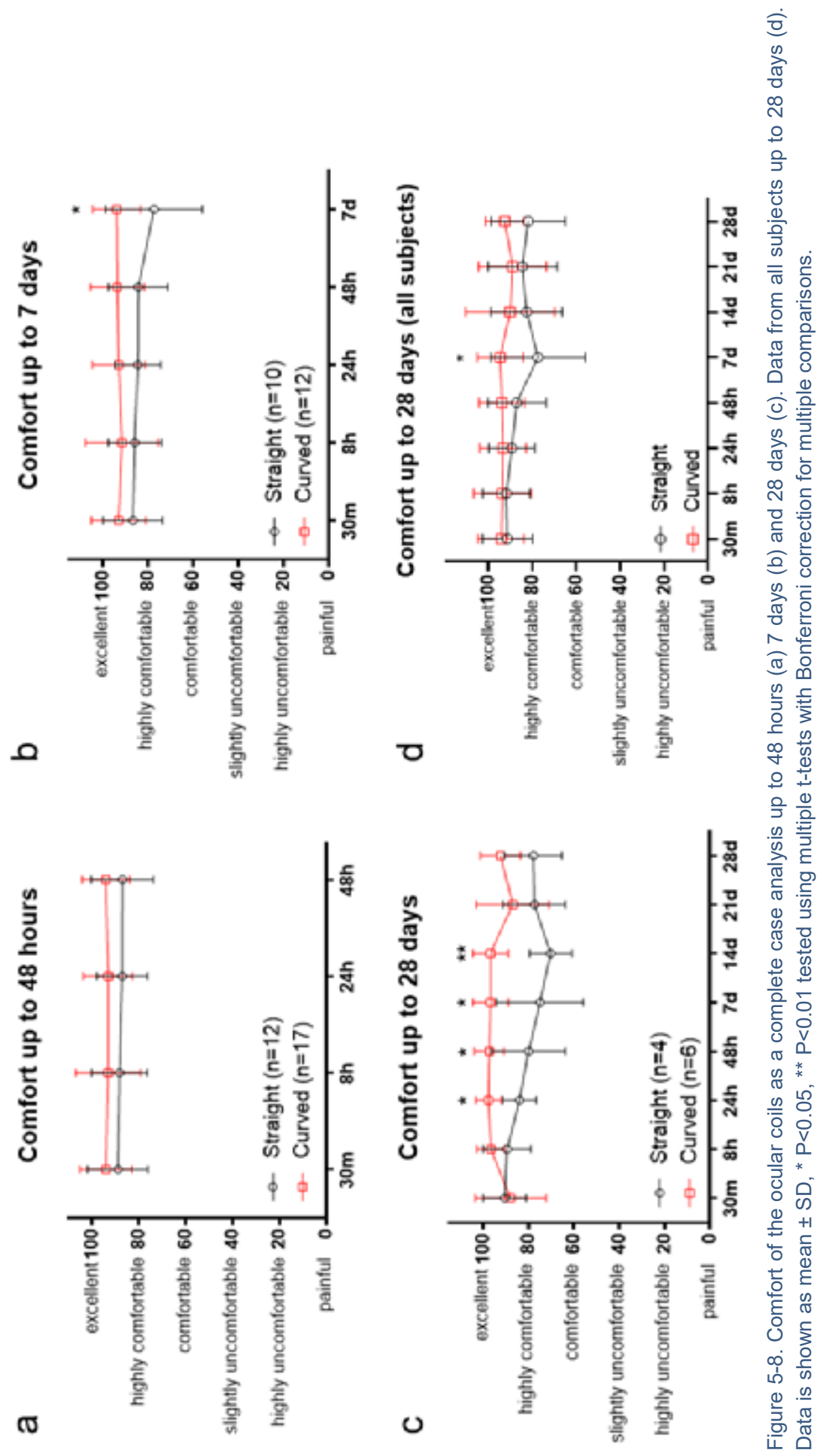



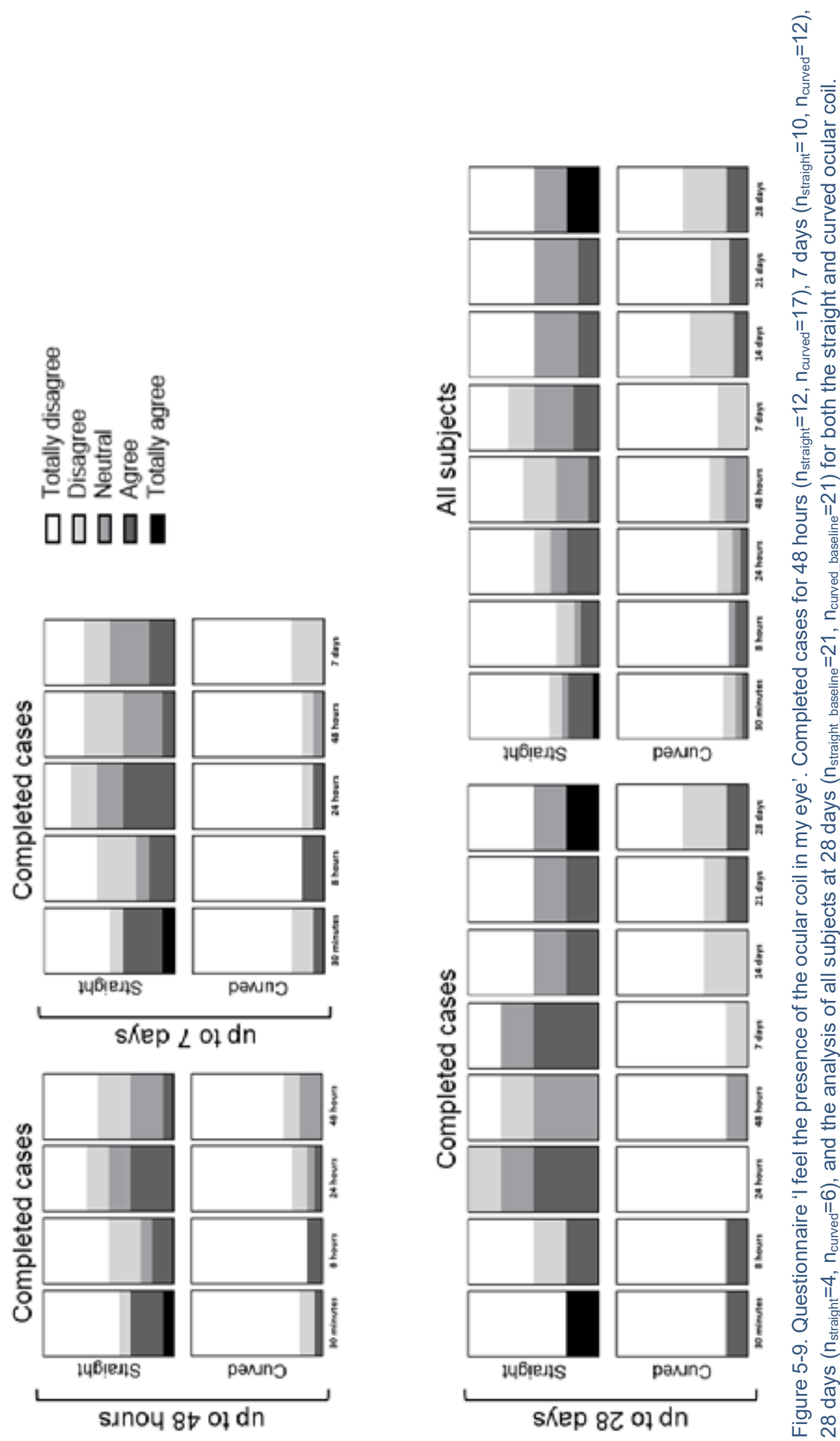

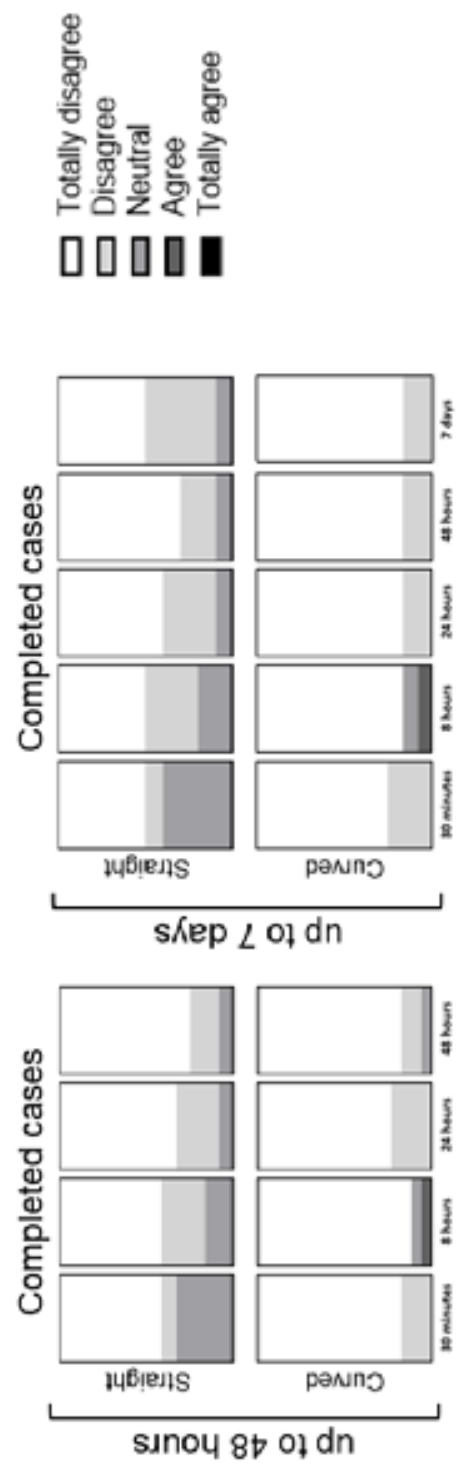

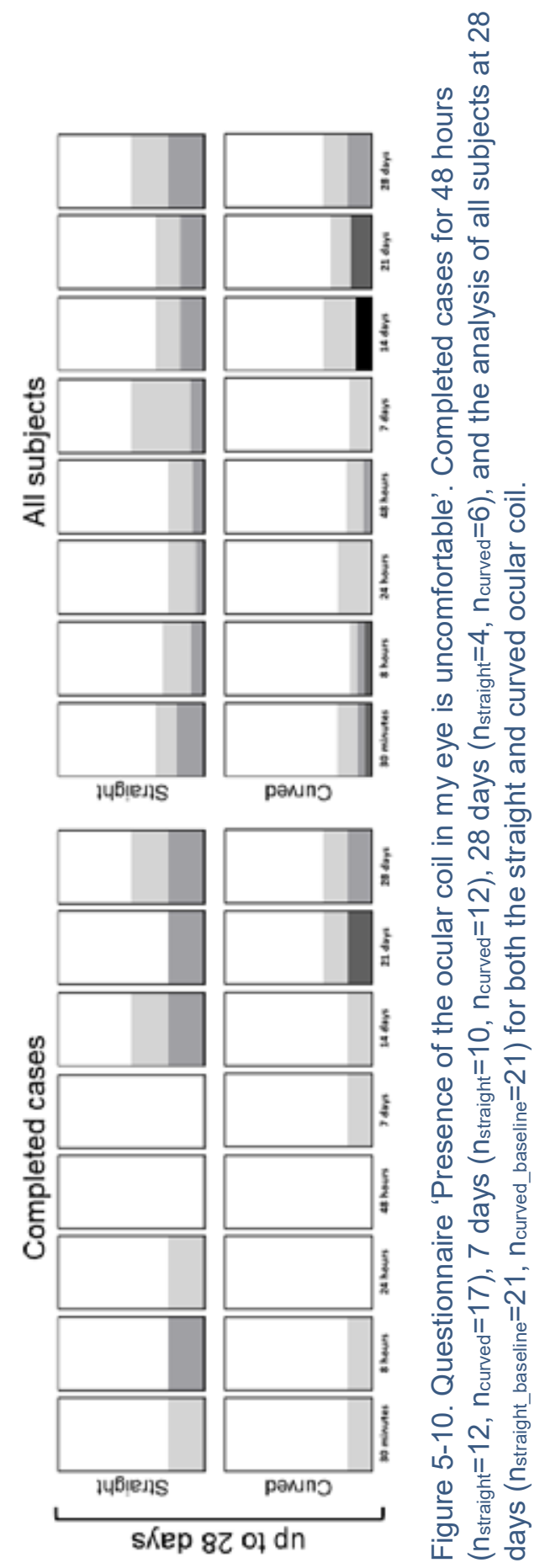


Subjects were asked whether their eyes teared more frequently while wearing the ocular coil. The majority of subjects did not experience increased tearing. One subject wearing the straight ocular coil went from "sometimes", to "often" and one went from "sometimes" to "continuously" after 30 minutes, however this returned to baseline level at 8 hours. Few subjects wearing the straight ocular coil reported a mild increase in tearing, whereas the curved ocular coil subjects stayed stable compared to baseline (figure 5-S4). Tear production was also objectively assessed using a Schirmer's tear production test (figure 5-11). In contrast to an increased tearing experience of a few subjects, no significant difference between the control eye and study eye was observed using the Schirmer's test. There was no significant change over time in both study arms.

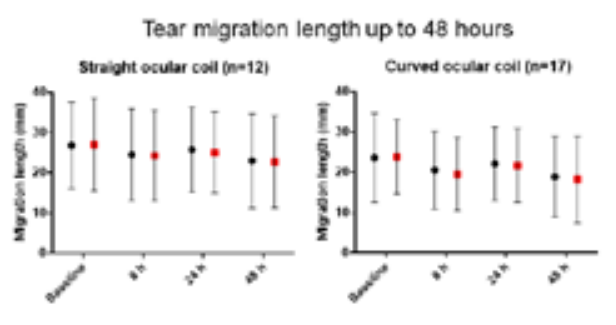

Tear migration length up to 28 days

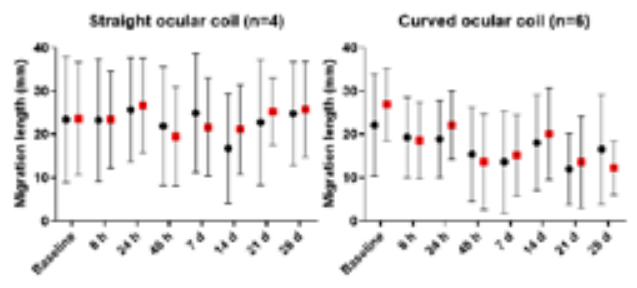

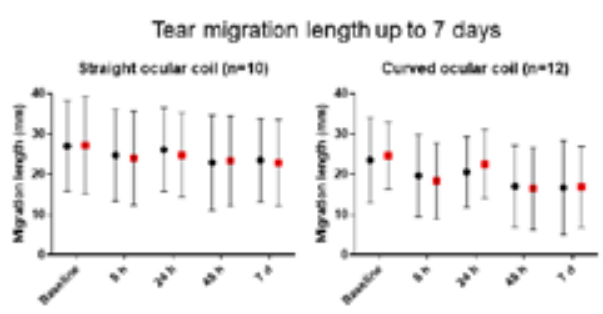

Tear migration length up to 28 days (all subjects)

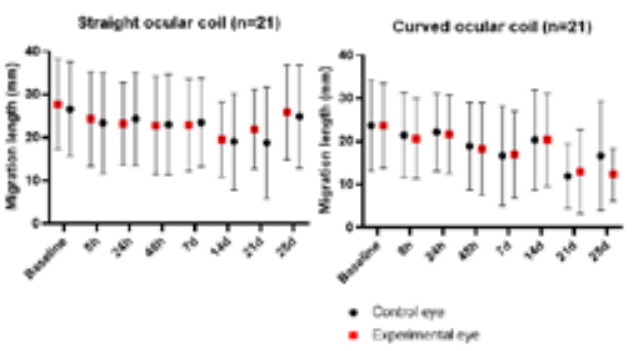

Figure 5-11. Schirmer's tear production test (II) for the study eye (red square) and control eye (black dot). Completed cases for 48 hours ( $\left.n_{\text {straight }}=12, n_{\text {curved }}=17\right), 7$ days $\left(n_{\text {straight }}=10, n_{\text {curved }}=12\right), 28$ days $\left(n_{\text {straight }}=4\right.$, $\left.\mathrm{n}_{\text {curved }}=6\right)$, and the analysis of all subjects at 28 days $\left(n_{\text {straight_baseline }}=21, n_{\text {curved_baseline }}=21\right)$ for the straight and curved ocular coil.

\subsubsection{Adverse events}

All adverse events are shown in table 5-3. No serious adverse events were reported during the course of the study. Forty-three percent of the subjects (at both ocular coils) experienced migration of the ocular coil towards the caruncle (figure 5-S2b). Adverse events included corneal erosion (figure 5-S1), dislocation of the ocular coil, ocular irritation, transient blurred vision, painful or foreign body sensations, ocular discharge, and headache. Dislocation of the curved ocular coil towards the superior 
conjunctival fornix was observed in three (14.3\%) subjects (figure 5-S3a). Within these three cases, one dislocated ocular coil was removed while two ocular coils were repositioned.

Table 5-3. Adverse events association with wearing the ocular coil

\begin{tabular}{|lcc|}
\hline & $\begin{array}{c}\text { Straight coil } \\
\mathbf{n}(\%)\end{array}$ & $\begin{array}{c}\text { Curved coil } \\
\mathbf{n}(\%)\end{array}$ \\
$\begin{array}{l}\text { Ocular adverse events } \\
\text { Ocular irritation }\end{array}$ & $1(5 \%)$ & - \\
Corneal erosion & $1(5 \%)$ & - \\
Transient blurred vision & $1(5 \%)$ & $1(5 \%)$ \\
$\begin{array}{l}\text { Painful or foreign body } \\
\text { sensations }\end{array}$ & $1(5 \%)$ & $9(43 \%)$ \\
$\begin{array}{l}\text { Dislocation of the ocular } \\
\text { coil toward the caruncle }\end{array}$ & $9(43 \%)$ & $3(14 \%)$ \\
$\begin{array}{l}\text { Dislocation of the ocular } \\
\text { coil to the superior fornix }\end{array}$ & - & $1(5 \%)$ \\
Ocular discharge & $3(14 \%)$ & - \\
\hline $\begin{array}{l}\text { Systemic adverse events } \\
\text { Headache }\end{array}$ & $1(5 \%)$ & \\
\hline
\end{tabular}

\subsection{Discussion}

This study gives a detailed insight into safety and comfort of the ocular coil. Safety and comfort are essential for a new drug delivery device in order to serve as a functional alternative to eye drops and assure high compliance. In a pilot study, 5 healthy subjects wore one ocular coil (filled with hydrogel-coated placebo filaments in its inner lumen) for 2 hours. Although the subjects felt the presence of the ocular coil in the conjunctival fornix, the coil was not scored as unpleasant (mean comfort score of $2.2 \pm 1.2$ on a scale from $1=$ very comfortable, to $5=$ uncomfortable).[17] In addition, the eye did not show signs of ocular irritation.[17]

In this study, two new versions of the ocular coil (filled with placebo microspheres) were tested. A small number of subjects felt the presence of the ocular coil in the conjunctival fornix. This number increased over time as the subjects became more aware of the straight ocular coil. In contrast, the curved ocular coil was only minimally felt in the fornix. We therefore questioned the subjects whether presence of the ocular coil was uncomfortable and whether the subjects were hindered in their daily tasks by the ocular coil. Presence of the ocular coil was felt but wearing the ocular coil was not considered annoying nor did it hinder the subjects during their daily tasks. Although both ocular coils were considered comfortable, the curved coil 
provided a more stable comfort score over the full duration of the study. Safety of the ocular coil is another important factor. To exclude drug related side effects of a drug delivery device, the ocular coil was tested with placebo microspheres in healthy subjects. One of the main symptoms indicating ocular irritation would be conjunctival hyperemia.[24] Hyperemia was scored using the Efron's grading scale.[21] Subtle variations in hyperemia were observed during the study. However, placement of the ocular coil did not result in acute hyperemia, nor was there chronic irritation resulting in an increase in hyperemia after wearing the ocular coil for multiple weeks. On day 28 , one of the subjects rubbed his eye which resulted in a hyposphagma (figure 5-S3). It is difficult to conclude whether the hyposphagma occurred due to the presence of the ocular coil or only due to eye rubbing. Similarly, it was hard to judge whether the corneal erosion in another subject was due to dislocation of the ocular coil or to a twig from a tree that the subject accidentally got in his eye. In both cases, we cannot rule out that dislocation of the coil contributed to the occurrence of the corneal epithelial defects. The advantageous non-invasive (and mobile) nature of the ocular coil, therefore, also has its drawbacks impeding future clinical applications. The risk of complications due to dislocation could be minimized by increasing further the biocompatibility of the coil (e.g. modify the coating to decrease the friction of the surface), and by optimizing the device's design in order to prevent (sharp) edges and irregular interfaces.

The Efron's grading scale was created to evaluate contact lens related complications, it enabled us to carefully score and track ocular changes related to the ocular coil. Our study showed an average conjunctival hyperemia score of 0.78 \pm 0.82 and $1.00 \pm 1.04$ for subjects wearing the straight ocular coil and the curved ocular coil over a period of 28 days, respectively. These results are comparable to the average conjunctival hyperemia scores that were observed in 2 cohorts testing contact lens materials in 20 healthy adult contact lens wearers (i.e. $0.75 \pm 0.19$ and $0.94 \pm 0.25$ ).[25] Objective scoring of ocular hyperemia, however, remains difficult. Inter- and intra-observer differences are inevitable, particularly in large multi-center studies.[26] Therefore, our group is developing an automated computer program for objective redness scoring of slit lamp images.[27]

According to the Efron grading scale a neovascularization score of 1 was also present in 7 subjects at baseline, a finding clearly not related to the presence of the coil. In these seven subjects, no increase in neovascularization was noted during the study. An increase in vascularization from grade 0 to grade 1 was seen in 5 subjects, remained stable in 7 of these subjects, and disappeared in 6 of the subjects. Neovascularization was not accompanied by other symptoms or complaints. We therefore hypothesize that the variation might be contributed due to differences in subjective grading. To rule out that the changes are not caused by the coil itself but due to variations in grading, an objective neovascularization measurement system 
could be helpful in avoiding the variations inherent of subjective grading systems. Retention time of the ocular coil in the eye was lower than expected. We noticed that the majority of the subjects lost the ocular coil when they were manipulating their eye (lids, e.g. rubbing or washing). In some subjects, loss of the ocular coil occurred while sleeping. Introducing an ocular eye shield at night did not improve retention. Redesigning the ocular coil from straight to curved to lower tension on the tissue in the fornix did not increase the average retention time $(10.0 \pm 11.1$ days to $13.3 \pm$ 11.7 days) but improved (although not significantly) the median retention time (from 5 to 12 days). However, for a 48 hours period a retention time of $81 \%$ could be achieved using the curved ocular coil.

Devices with other shapes have similar retention issues. The rod-shaped ocular drug delivery device (Ocufit SR, 25 - $30 \mathrm{~mm}$ length, $1.9 \mathrm{~mm}$ diameter) could be retained for 2 weeks in the superior conjunctival fornix in $70 \%$ of the cases.[28] Although these retention times are higher than ours $(43 \%$ of cases for the straight coil and $48 \%$ of cases for the curved coil over a 2-week period), we prefer placement of the device in the inferior conjunctival fornix in order to lower the risk for causing corneal damage following blinking of the upper eyelid. Furthermore, placement of the ocular coil in the inferior fornix appears not to interfere with eye muscle movements.[18]

Another study, performed by Katz et al., tested retention of a dissolvable rod and a dissolvable oval shaped drug delivery device for 24 hours tested for 7 days (a new device every day). They found that a rod like shape is beneficial over an oval shape. Furthermore, $60 \%$ of their drug delivery devices were lost upon, or within 1 hour after arising, when subjects inadvertently rubbed their eyes.[29] In our study, six subjects lost the ocular coils during sleep (15\%).

More recently, the bimatoprost ring (also known as Helios ${ }^{\mathrm{TM}}$ ) (Allergan, Dublin, IR) was developed. This ring is inserted in the superior and inferior fornices around the bulbus. The retention time of the bimatoprost ring was $93 \%$ at 12 weeks, and $88.5 \%$ at 6 months.[30] However, the retention time in their study was defined as maintenance of the insert without requiring physician re-intervention.[30] In all cases, patients were aware of dislodgement of the bimatoprost ring.[31] Therefore, patients were instructed to reinsert the bimatoprost ring themselves, which resulted in a learning curve, increasing retention time (from $88 \%$ to $97 \%$ in 6 to 7 months).[31] In contrast, our subjects were instructed not to reinsert the ocular coil after loss. Furthermore, when dislocation of the ocular coil was observed by the investigators, $24 \%$ of subjects were not aware of this dislocation. Retention time of small devices for the inferior conjunctival fornix is lower compared to ring-like structures.[30,31] This may be a problem with any single-fornix ocular devices. Despite different shapes, all these types of (relatively large) drug delivery devices thus seem to share similar problems with dislocation and loss from the eye.

Given the acceptable retention time of $81 \%$ over the 48 hours period, a curved coil 
may be suitable to use for perioperative application during cataract surgery. Currently, there is growing interest in so-called dropless cataract surgery, where drug-loaded devices can provide adequate medical treatment to prevent postoperative inflammation.[32-34] In the US, Imprimis Pharmaceuticals (San Diego, CA, USA) developed TriMoxi (less drops $^{\circledR}$ ) and TriMoxiVanc (dropless ${ }^{\circledR}$ ), two compounded injections that consists of Triamcinolone and Moxifloxacin for perioperative use.[32,34] They estimated that as such the use of post-operative drops can be avoided in more than $90 \%$ of patients. [35] However, one must take into account the obstructed vision (a "cloud" or "plume") during the first days to week post-operatively.[32]

Another perioperative solution developed by Omerios Coorperation (Seattle, WA, USA) is Omidria ${ }^{\circledR}$. Omidria ${ }^{\circledR}$ contains phenylephrine $(1 \%)$ and ketorolac $(0.3 \%)$ and is used in the irrigation fluid during surgery. Omidria ${ }^{\circledR}$ stabilizes mydriasis and reduces post-operative pain.[36] However, Omidria ${ }^{\circledR}$ is not intended as prophylaxis for cystoid macular edema. A third injectable is Dexycu ${ }^{\mathrm{TM}}$, developed by EyePoint Pharmaceutics (Watertown, MA, USA). Dexycu ${ }^{\mathrm{TM}}$ is a $9 \%$ dexamethasone suspension to be injected peri-operatively after insertion of the intraocular lens and reduces post-operative inflammation.[37]

Recently, Ocular Therapeutix (Bedford, MA, USA) brought Dextenza ${ }^{\circledR}$ on the market, a $0.7 \mathrm{mg}$ dexamethasone containing punctum plug to prevent post-operative inflammation.[33] Two prospective multicenter studies observed a reduction in ocular pain and inflammation compared to a placebo device.[38] Ninety-six percent of patients were satisfied with the use of Dextenza ${ }^{\circledR}$ and $88 \%$ would want to use the insert again after ocular surgery.[39] These results demonstrate that there is market potential for non-invasive drug delivery devices.

With a retention time of $81 \%$ after 48 hours, the curved ocular coil would be suitable to use in the early post-operative phase after ocular surgery. Further studies are needed to investigate its efficacy and applicability.

\subsection{Conclusion}

This single-center intervention study provides an overview of the safety and comfort of two versions of the ocular coil. The current study indicates a high comfort profile of both ocular coils designs. Whereas safety of the curved ocular coil seems higher than the straight ocular coil because of the occurred adverse events. Retention time of the ocular coils, however, was lower than expected for the 7 days and 28 days periods, but satisfactory for a 48-hour period. This would make the current design suitable for drug delivery in a burst release mode in the early post-operative phase in surgical procedures that elicit a low to moderate inflammatory response like cataract surgery. This potential application will need further investigation. 


\subsection{References}

1. Wielders, L.H.P., Schouten, J., and Nuijts, R., Prevention of macular edema after cataract surgery. Curr Opin Ophthalmol, 2018. 29(1): p. 48-53.

2. Wielders, L.H., Schouten, J.S., Aberle, M.R., Lambermont, V.A., van den Biggelaar, F.J., Winkens, B., Simons, R.W., and Nuijts, R.M., Treatment of cystoid macular edema after cataract surgery. J Cataract Refract Surg, 2017. 43(2): p. 276-284.

3. Wielders, L.H.P., Schouten, J., Winkens, B., van den Biggelaar, F., Veldhuizen, C.A., Findl, O., Murta, J.C.N., Goslings, W.R.O., Tassignon, M.J., Joosse, M.V., Henry, Y.P., Rulo, A.H.F., Guell, J.L., Amon, M., Kohnen, T., Nuijts, R., and Group, E.P.S., European multicenter trial of the prevention of cystoid macular edema after cataract surgery in nondiabetics: ESCRS PREMED study report 1. J Cataract Refract Surg, 2018. 44(4): p. 429-439.

4. Urtti, A., Challenges and obstacles of ocular pharmacokinetics and drug delivery. Adv Drug Deliv Rev, 2006. 58(11): p. 1131-1135.

5. $\quad$ Farkouh, A., Frigo, P., and Czejka, M., Systemic side effects of eye drops: a pharmacokinetic perspective. Clin Ophthalmol, 2016. 10: p. 2433-2441.

6. Kaszli, F.A. and Krieglstein, G.K., Tear film deficiencies pharmacology of eye drops and toxicity. Curr Opin Ophthalmol, 1996. 7(4): p. 12-16.

7. Palmer, R.M. and Kaufman, H.E., Tear film, pharmacology of eye drops, and toxicity. Curr Opin Ophthalmol, 1995. 6(4): p. 11-16.

8. Ramsay, E., Del Amo, E.M., Toropainen, E., Tengvall-Unadike, U., Ranta, V.P., Urtti, A., and Ruponen, M., Corneal and conjunctival drug permeability: Systematic comparison and pharmacokinetic impact in the eye. Eur J Pharm Sci, 2018. 119: p. 83-89.

9. Del Amo, E.M., Rimpela, A.K., Heikkinen, E., Kari, O.K., Ramsay, E., Lajunen, T., Schmitt, M., Pelkonen, L., Bhattacharya, M., Richardson, D., Subrizi, A., Turunen, T., Reinisalo, M., Itkonen, J., Toropainen, E., Casteleijn, M., Kidron, H., Antopolsky, M., Vellonen, K.S., Ruponen, M., and Urtti, A., Pharmacokinetic aspects of retinal drug delivery. Prog Retin Eye Res, 2017. 57: p. 134-185.

10. Pelkonen, L., Tengvall-Unadike, U., Ruponen, M., Kidron, H., Del Amo, E.M., Reinisalo, M., and Urtti, A., Melanin binding study of clinical drugs with cassette dosing and rapid equilibrium dialysis inserts. Eur J Pharm Sci, 2017. 109: p. 162-168.

11. Olthoff, C.M., Hoevenaars, J.G., van den Borne, B.W., Webers, C.A., and Schouten, J.S. Prevalence and determinants of non-adherence to topical hypotensive treatment in Dutch glaucoma patients. Graefes Arch Clin Exp Ophthalmol, 2009. 247(2): p. 235-243.

12. An, J.A., Kasner, O., Samek, D.A., and Levesque, V., Evaluation of eyedrop administration by inexperienced patients after cataract surgery. J Cataract Refract Surg, 2014. 40(11): p. 18571861.

13. Bertens, C.J.F., Gijs, M., van den Biggelaar, F., and Nuijts, R., Topical drug delivery devices: A review. Exp Eye Res, 2018. 168: p. 149-160.

14. Pijls, R.T., Hanssen, H.H., Nuijts, R.M., and Koole, L.H., Flexible coils with a drug-releasing hydrophilic coating: a new platform for controlled delivery of drugs to the eye? Biomed Mater Eng, 2004. 14(4): p. 383-393.

15. Pijls, R.T., Sonderkamp, T., Daube, G.W., Krebber, R., Hanssen, H.H., Nuijts, R.M., and Koole, L.H., Studies on a new device for drug delivery to the eye. Eur J Pharm Biopharm, 2005. 59(2): p. 283-288.

16. Pijls, R.T., Hanssen, H.H., Nuijts, R.M., Daube, G.W., and Koole, L.H., In vivo tolerance and kinetics of a novel ocular drug delivery device. J Control Release, 2006. 116(2): p. e47-49.

17. Pijls, R.T., Cruysberg, L.P., Nuijts, R.M., Dias, A.A., and Koole, L.H., Capacity and tolerance of a new device for ocular drug delivery. Int J Pharm, 2007. 341(1-2): p. 152-161.

18. Bertens, C.J.F., Martino, C., van Osch, M.C., Lataster, A., Dias, A., van den Biggelaar, F., Tuinier, R., Nuijts, R., and Gijs, M., Design of the ocular coil, a new device for non-invasive drug delivery. Eur J Pharm Biopharm, 2020. 150: p. 120-130.

19. Ferris, F.L., 3rd, Kassoff, A., Bresnick, G.H., and Bailey, I., New visual acuity charts for clinical research. Am J Ophthalmol, 1982. 94(1): p. 91-96.

20. National Eye Institute. Visual Function Questionnaire (VFQ-25), https://www.rand.org/healthcare/surveys_tools/vfq.html, (accessed 19 Nov 2019), online.

21. Efron, N., Grading scales for contact lens complications. Ophthalmic Physiol Opt, 1998. 18(2): p. $182-186$. 
22. Bron, A.J., Evans, V.E., and Smith, J.A., Grading of corneal and conjunctival staining in the context of other dry eye tests. Cornea, 2003. 22(7): p. 640-650.

23. Jabs, D.A., Nussenblatt, R.B., Rosenbaum, J.T., and Standardization of Uveitis Nomenclature Working, G., Standardization of uveitis nomenclature for reporting clinical data. Results of the First International Workshop. Am J Ophthalmol, 2005. 140(3): p. 509-516.

24. Cronau, H., Kankanala, R.R., and Mauger, T., Diagnosis and management of red eye in primary care. Am Fam Physician, 2010. 81(2): p. 137-144.

25. Santodomingo-Rubido, J., The comparative clinical performance of a new polyhexamethylene biguanide- vs a polyquad-based contact lens care regime with two silicone hydrogel contact lenses. Ophthalmic Physiol Opt, 2007. 27(2): p. 168-173.

26. Peterson, R.C. and Wolffsohn, J.S., Objective grading of the anterior eye. Optom Vis Sci, 2009. 86(3): p. 273-278.

27. Sirazitdinova, E., Gijs, M., Bertens, C.J.F., Berendschot, T., Nuijts, R., and Deserno, T.M., Validation of Computerized Quantification of Ocular Redness. Transl Vis Sci Technol, 2019. 8(6): p. 31.

28. Saettone, M.F. and Salminen, L., Ocular inserts for topical delivery. Advanced Drug Delivery Reviews, 1995. 16(1): p. 95-106.

29. Katz, I.M. and Blackman, W.M., A soluble sustained-release ophthalmic delivery unit. Am J Ophthalmol, 1977. 83(5): p. 728-734.

30. Brandt, J.D., Sall, K., DuBiner, H., Benza, R., Alster, Y., Walker, G., Semba, C.P., and Collaborators, Six-Month Intraocular Pressure Reduction with a Topical Bimatoprost Ocular Insert: Results of a Phase II Randomized Controlled Study. Ophthalmology, 2016. 123(8): p. 1685-1694.

31. Brandt, J.D., DuBiner, H.B., Benza, R., Sall, K.N., Walker, G.A., Semba, C.P., and Collaborators, Long-term Safety and Efficacy of a Sustained-Release Bimatoprost Ocular Ring. Ophthalmology, 2017. 124(10): p. 1565-1566.

32. Stringham, J.D., Flynn, H.W., Jr., Schimel, A.M., and Banta, J.T., Dropless Cataract Surgery: What Are the Potential Downsides? Am J Ophthalmol, 2016. 164: p. viii-x.

33. Rhee, M.K. and Mah, F.S., Cataract Drug Delivery Systems (Dropless vs. Nondropless Cataract Surgery). Int Ophthalmol Clin, 2016. 56(3): p. 117-136.

34. Lindstrom, R.L., Galloway, M.S., Grzybowski, A., and Liegner, J.T., Dropless Cataract Surgery: An Overview. Curr Pharm Des, 2017. 23(4): p. 558-564.

35. Barnet Dulaney Perkins eye center. $h$ ttps://www.goodeyes.com/portal/articles/trimoxi-cataractsurgery/, (accessed 15 Apr 2020), online.

36. Donnenfeld, E.D., Whitaker, J.S., Jackson, M.A., and Wittpenn, J., Intracameral ketorolac and phenylephrine effect on intraoperative pupil diameter and postoperative pain in cataract surgery. J Cataract Refract Surg, 2017. 43(5): p. 597-605.

37. Donnenfeld, E.D., Solomon, K.D., and Matossian, C., Safety of IBI-10090 for inflammation associated with cataract surgery: Phase 3 multicenter study. J Cataract Refract Surg, 2018. 44(10): p. 1236-1246.

38. Brooks, C.C., Jabbehdari, S., and Gupta, P.K., Dexamethasone 0.4mg Sustained-Release Intracanalicular Insert in the Management of Ocular Inflammation and Pain Following Ophthalmic Surgery: Design, Development and Place in Therapy. Clin Ophthalmol, 2020. 14: p. 89-94.

39. Gira, J.P., Sampson, R., Silverstein, S.M., Walters, T.R., Metzinger, J.L., and Talamo, J.H., Evaluating the patient experience after implantation of a $0.4 \mathrm{mg}$ sustained release dexamethasone intracanalicular insert (Dextenza): results of a qualitative survey. Patient Prefer Adherence, 2017. 11: p. 487-494. 


\subsection{Supplementary figures}

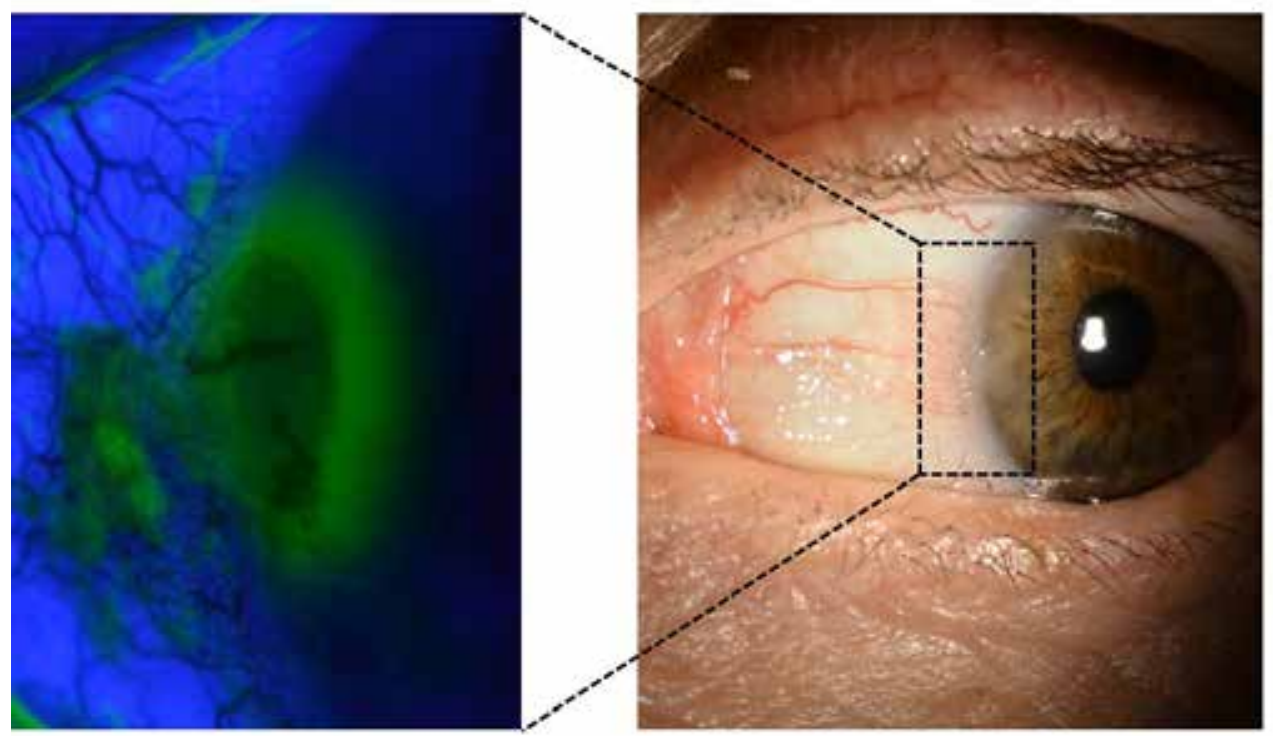

Figure 5-S1. Corneal erosion due to a twig in the eye of a subject (unrelated to the ocular coil). Slit lamp images at $6.3 x$ magnification and insert at $16 x$ magnification stained with fluorescein.
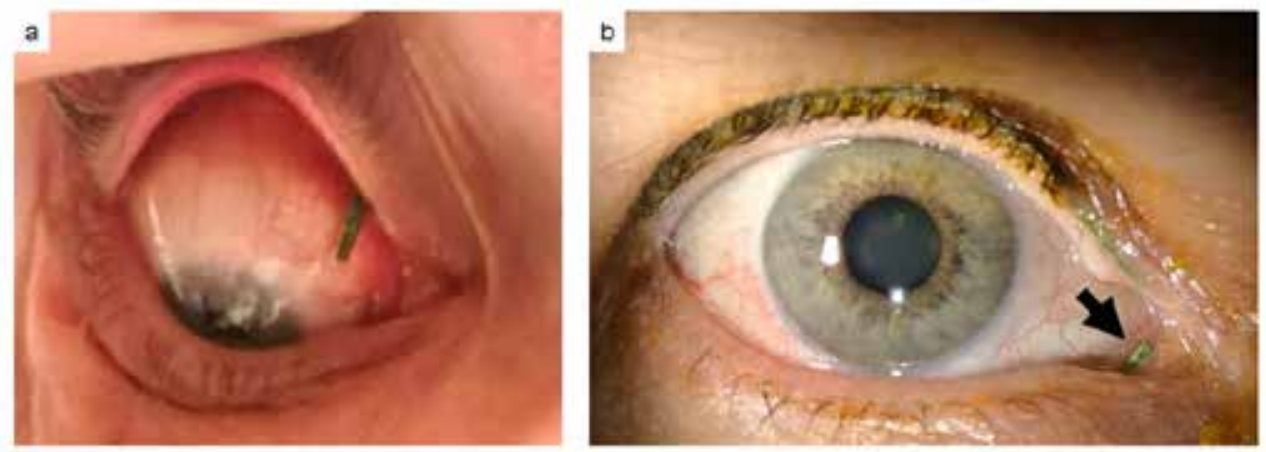

Figure 5-S2. (a) Dislocation of the curved ocular coil to the superior conjunctival fornix, and (b) migration of the ocular coil to the caruncle (indicated with the black arrow). Photograph (a) was taken with an iPhone $X R$. 

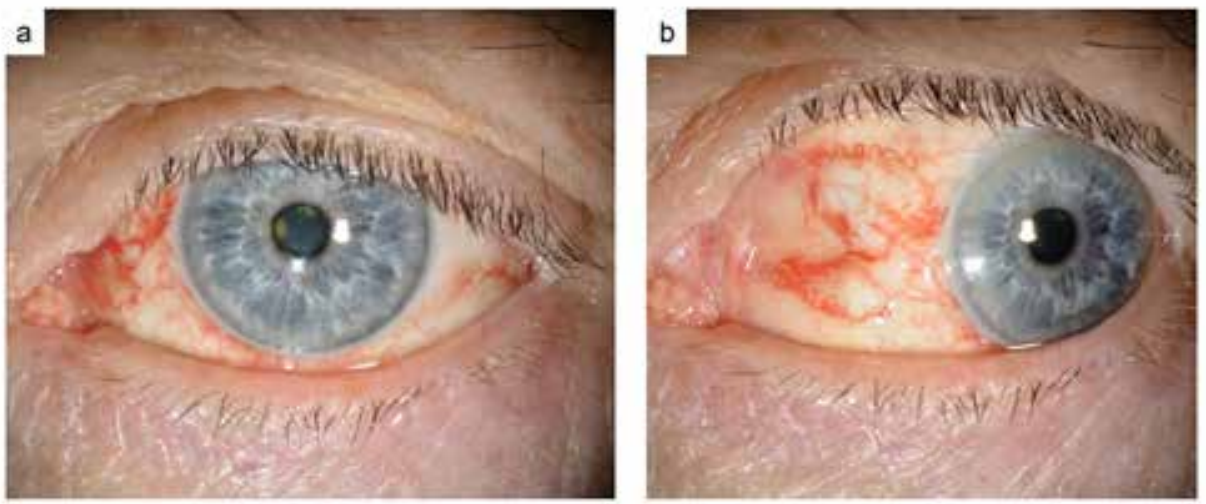

Figure 5-S3. Photograph of a hyposphagma (a) gazing forward, and (b) left gaze direction.
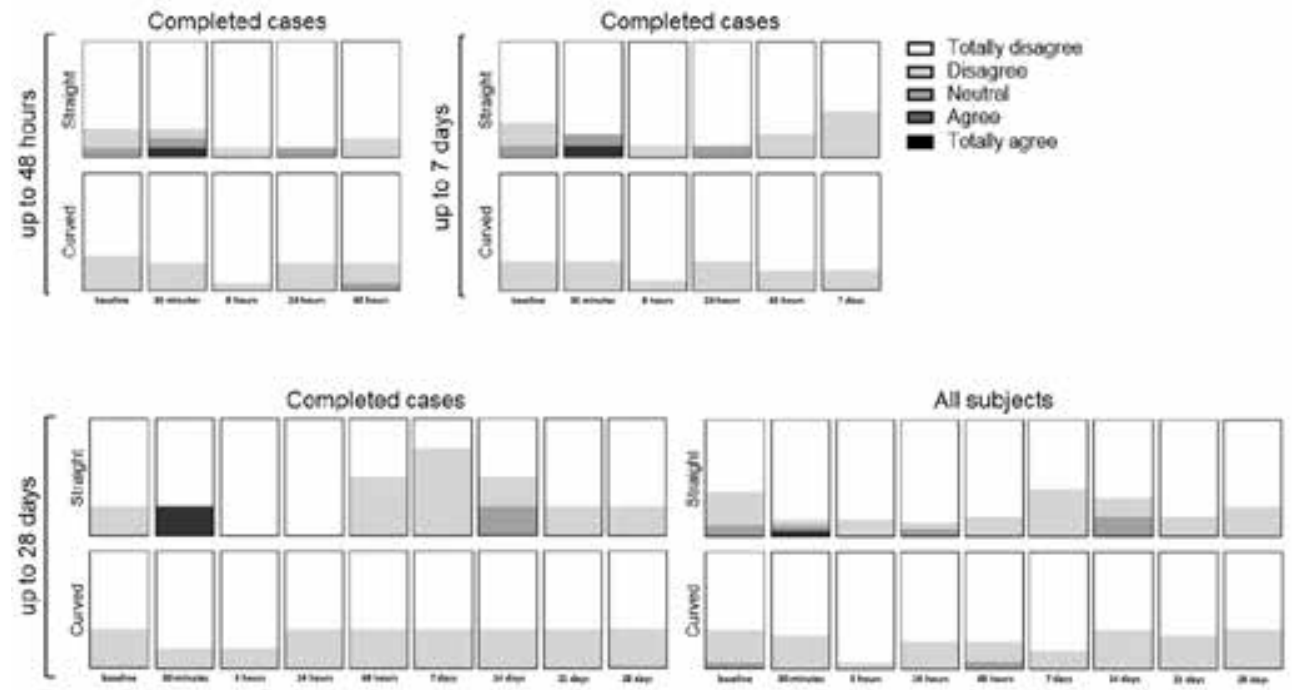

Figure 5-S4. Questionnaire 'My eye has a high tear production'. Completed cases for 48 hours $\left(\mathrm{n}_{\text {straight }}=12\right.$, $\left.n_{\text {curved }}=17\right), 7$ days $\left(n_{\text {straight }}=10, n_{\text {curved }}=12\right), 28$ days $\left(n_{\text {straight }}=4, n_{\text {curved }}=6\right)$, and the analysis of all subjects at 28 days $\left(n_{\text {straight_baseline }}=21, n_{\text {curved_baseline }}=21\right.$ ) for both the straight and curved ocular coil. 
Chapter 5

Table 5-S1. Additional questions VFQ25 version 2001.

4a Was pain or discomfort caused by $\circ$ Yes wearing contact lenses? $\quad \circ$ No

4b Was pain or discomfort caused by ocular $\bigcirc$ Yes surgery? $\circ$ No

4c Was pain or discomfort caused by use of $\bigcirc$ Yes ocular drugs e.g. eye drops or ointments? $\quad \circ$ No

4d Was pain or discomfort caused by an $\circ$ Yes allergic reaction e.g. hay fever, cat or dog $\bigcirc$ No allergy?

$4 \mathrm{e} \quad$ (if yes on question 4a) Do you wear $\circ$ Yes contact lenses to improve your eyesight? $\quad$ No (use 'no' for cosmetic reasons)

$4 \mathrm{f} \quad$ Why do you not use contact lenses?
- My eyesight is good therefore I do not need correction.
- I prefer glasses
- I cannot wear contact lenses due to ocular irritation or discomfort

Questions were inserted at PART 1, after question 4, if 'yes' on question 4. (Translated from Dutch to English). 
Table 5-S2. Questionnaire during follow-up visits (30m, 8h, 24h, 48h, 7d, 14d, 21d, and 28d), translated from the Dutch version.

The ocular coil is not properly located in my eye.

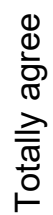

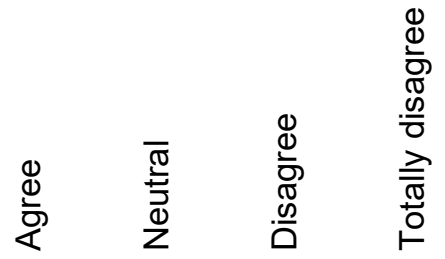

I feel the presence of the ocular coil in my eye.

Presence of the ocular coil is uncomfortable

1

2

3

4

5

Wearing the ocular coil made my vision blurry

Wearing the ocular coil lowered my visual acuity

1

2

3

4

5

Wearing the ocular coil made me close my eyes more often

Wearing the ocular coil hinders me in during daily tasks

$\begin{array}{lllll}1 & 2 & 3 & 4 & 5 \\ 1 & 2 & 3 & 4 & 5 \\ 1 & 2 & 3 & 4 & 5 \\ 1 & 2 & 3 & 4 & 5 \\ 1 & 2 & 3 & 4 & 5\end{array}$

\begin{tabular}{|c|c|c|c|c|}
\hline & $\frac{\grave{d}}{Z}$ & 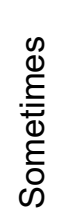 & $\underset{\frac{c}{d}}{\stackrel{c}{ \pm}}$ & 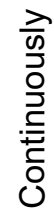 \\
\hline My eye has a high tear production & 1 & 2 & 3 & 4 \\
\hline $\begin{array}{l}\text { I have the intention to rub my eyes more } \\
\text { often }\end{array}$ & 1 & 2 & 3 & 4 \\
\hline My eye itches & 1 & 2 & 3 & 4 \\
\hline My eye hurts & 1 & 2 & 3 & 4 \\
\hline I have eyestrain & 1 & 2 & 3 & 4 \\
\hline $\begin{array}{l}\text { My eye feels irritated/It feels like there } \\
\text { is sand in my eye. }\end{array}$ & 1 & 2 & 3 & 4 \\
\hline My eye feels burning & 1 & 2 & 3 & 4 \\
\hline
\end{tabular}


Which grade would you give the ocular coil, according to the scaling on the right?

Are there any other comments or remarks?

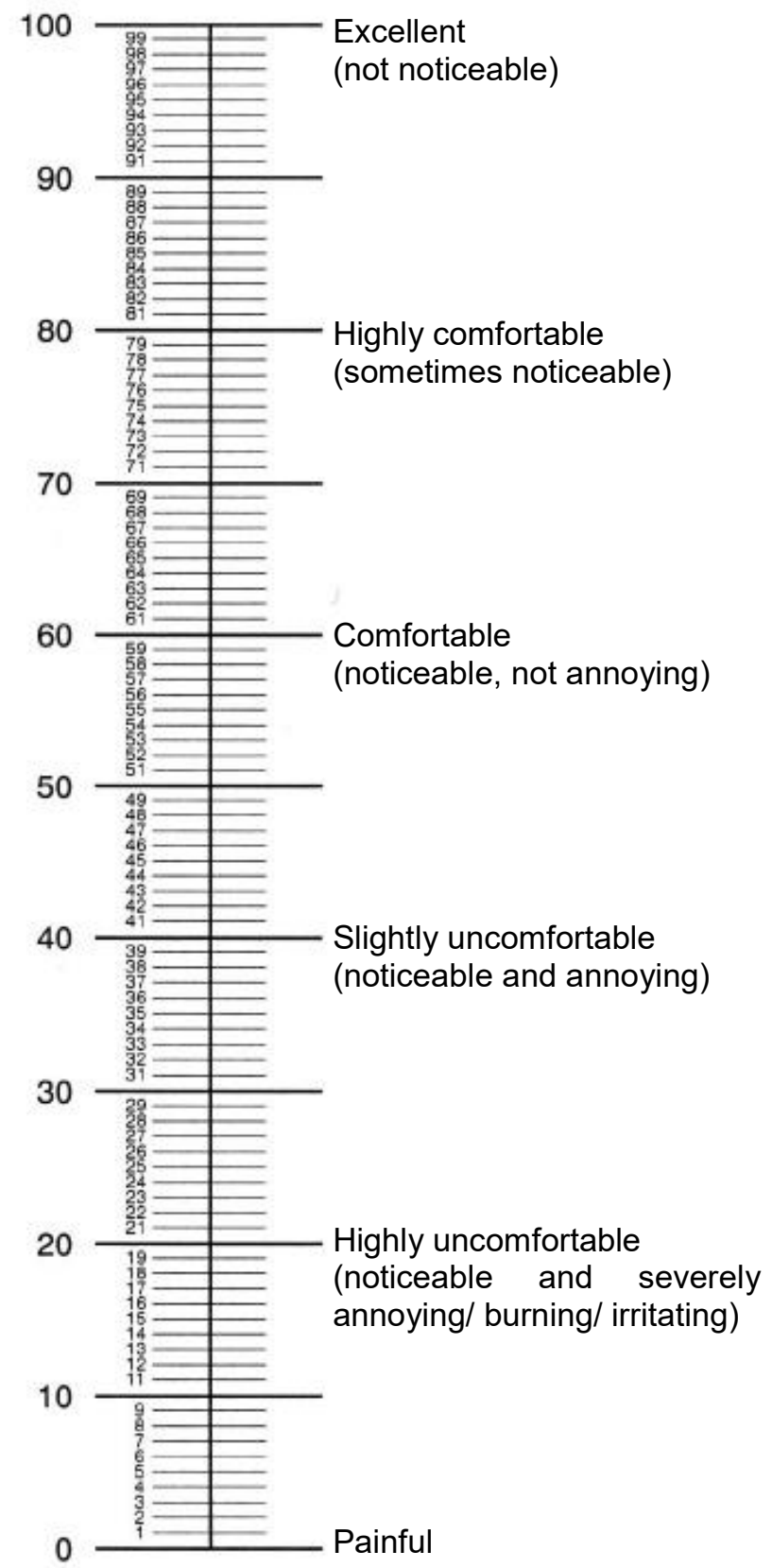


Safety and comfort of an innovative drug delivery device in healthy subjects 


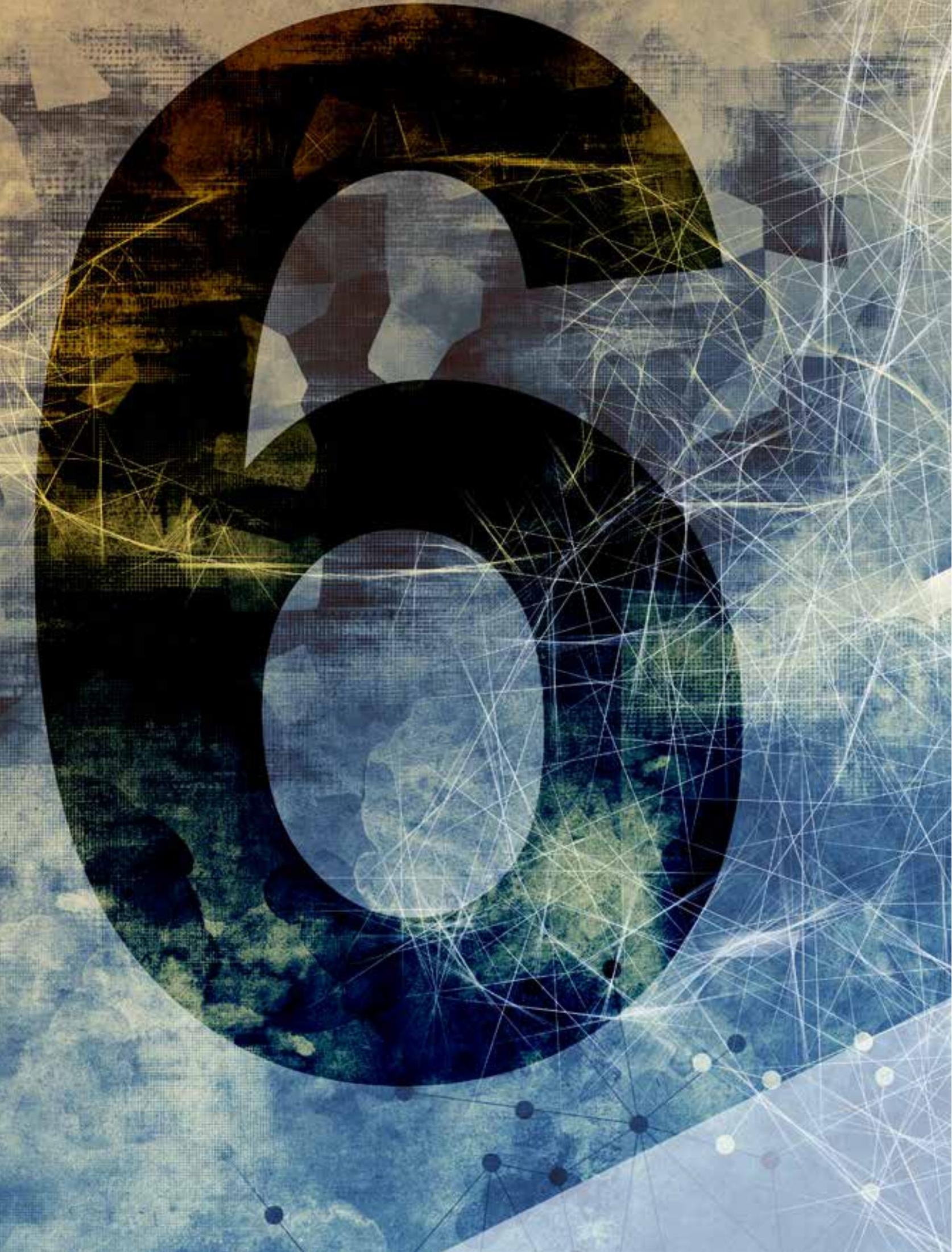


Chapter 6.

\section{Confocal Raman spectroscopy:}

evaluation of a honninvasive

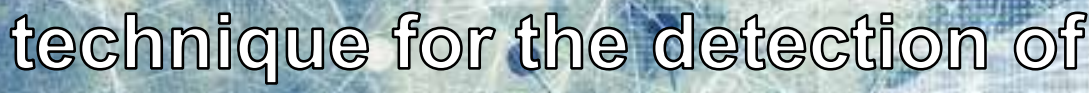

topically applied ke\{०rolac

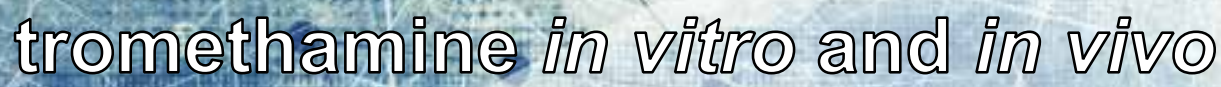

Christian J.F. Bertens, Shuo Zhang, Roel J. Erckens, Frank J.H.M. van den Biggelaar, Tos T.J.M. Bertendschot, Carroll A.B. Webers, Rudy M.M.A. Nuijts, and Marlies Gijs

International Journal of Pharmaceutics. 2019 Oct 30; Vol.570:118641.

DOI: $10.1016 / j . j$ jpharm.2019.118641 


\section{Abstract}

Current information about the pharmacokinetics of an ocular drug can only be achieved by invasive sampling. However, confocal Raman spectroscopy bears the potential to quantify drug concentrations non-invasively. In this project, we evaluated the detection and quantification of ocular ketorolac tromethamine levels with confocal Raman spectroscopy after topical administration.

Confocal Raman spectroscopy and high-performance liquid chromatography (HPLC) were compared in terms of sensitivity of detection. Enucleated pig eyes were treated with different concentrations of ketorolac. Hereafter, ketorolac concentrations in the aqueous humor of pig eyes were analyzed by confocal Raman spectroscopy and HPLC.

Subsequently, twelve rabbits were treated with Acular ${ }^{\mathrm{TM}}$ for four weeks. At several time points, ketorolac concentrations in aqueous humor of the rabbits were measured by confocal Raman spectroscopy followed by drawing an aqueous humor sample for HPLC analysis.

In ketorolac treated pig eyes, both ex vivo Raman spectroscopy as well as HPLC were able to detect ketorolac in a broad concentration range. However, in vivo confocal Raman spectroscopy in rabbits was unable to detect ketorolac in contrast to HPLC.

To conclude, confocal Raman spectroscopy has the capacity to detect ketorolac tromethamine in vitro, but currently lacks sensitivity for in vivo detection.

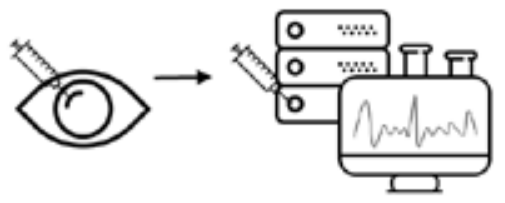

Invasive sampling, purification and HPLC analysis

VS.

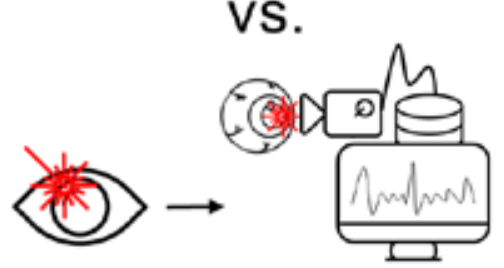

In vivo, non-invasive and realtime Raman detection
In vitro
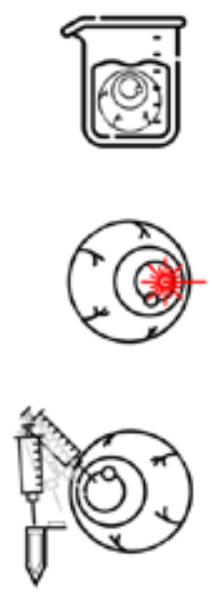

In vivo
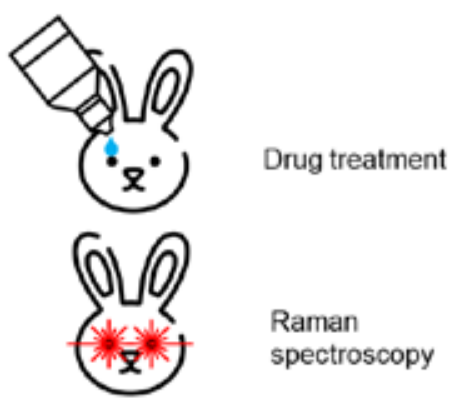

Raman

spectroscopy

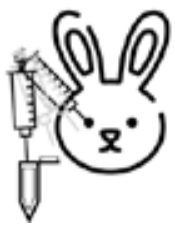

Cuvette analysis

- Raman spectroscops 


\subsection{Introduction}

Ocular pharmacokinetic studies investigate time- and dose dependent behavior of ophthalmic drugs. These studies are important to detect the maximum drug concentration $\left(\mathrm{C}_{\max }\right)$, the time to reach $\mathrm{C}_{\max }\left(\mathrm{T}_{\max }\right)$, half-life, and clearance of the drug. Based on those parameters, a dosage regimen can be created.[1] Evaluation of a pharmacokinetic profile should include assessment of systemic exposure (i.e., blood, plasma or serum levels) as well the distribution and levels in ocular tissues (e.g., cornea, iris, aqueous humor). Currently, the assessment of ocular pharmacokinetics is using tissues or fluids in a destructive test which comprises chemical pre-treatment followed by high-performance liquid chromatography (HPLC).[2] Besides extensive processing time, also sampling has been a challenge as ocular tissues or fluids cannot be harvested without interfering with the anatomical integrity of the eye (e.g., during intraocular surgery). Due to the limited accessibility of samples from humans, and the destructiveness of the method, pharmacokinetic research is relying on large quantities of animals, e.g. rabbits, dogs, pigs, and monkeys [3], because the eyes of these animals show similarities to human eyes.[4] Therefore, animal experiments have been widely criticized for both, ethical and economical reasons.[5]

A non-invasive pharmacokinetic assessment technique could resolve these issues. A technique that is potentially suitable for non-invasive detection of ocular pharmacokinetics is Raman spectroscopy. Raman spectroscopy identifies molecules, based on the specific inelastic scattering properties of their rotational and vibrational modes.[6-8] This technique enables real-time detection of molecules without pre-processing and damaging tissue. As such, the number of animals and its associated costs needed for ocular pharmacokinetic studies can be reduced. Compared to infrared (IR) spectroscopy, Raman spectroscopy bears the advantage that molecules do not have to possess a permanent dipole moment, therefore, more molecules can be detected. Furthermore, Raman spectroscopy is not affected by aqueous samples, whereas IR is absorbed intensively by water. Near-infrared (NIR) spectroscopy (NIRS) is, like Raman spectroscopy, not affected by aqueous samples. However, absorption bands tend to overlap in NIRS, which results in less accessible molecule-specific information, thus a lower specificity compared to Raman spectroscopy.[9]

Since Raman spectroscopy is a scattering technique, fiber-optics and remote sampling can be used. Samples can be measured directly in glass container or in case of pharmaceuticals, samples can be measured in original sachets. Because of a high spatial resolution, components from complex samples can be identified (e.g., cell-media components from a commercial recombinant-protein manufacturing process).[10] However, due to the weak nature of Raman scattering (1 in $10^{9}$ or 
$\left.10^{10}[11]\right)$, higher sensitivity of Raman spectroscopy requires higher power lasers as excitation source. Therefore, Raman spectroscopy is very suitable for chemical applications; but the photo-thermal effects in light-tissue interaction could cause concerns in vivo if the wrong wavelength is selected as emitting source. If the local temperature reaches up to $43.0^{\circ} \mathrm{C}$ hyperthermia will occur. A temperature up to $70^{\circ} \mathrm{C}$ could lead to tissue coagulation and welding. Higher temperatures (above $100^{\circ} \mathrm{C}$ ) cause vaporization and (above $300^{\circ} \mathrm{C}-450^{\circ} \mathrm{C}$ ) carbonization. [12] Another challenge for Raman spectroscopy is interference from fluorescence. Biological samples often emit fluorescence signals in the same wavelength range as Raman signals.[2] Finally, data processing of Raman spectroscopy is a challenging task. Since there is no well-accepted standard procedure in data processing for bio-spectroscopy yet[13], each (animal) model needs a specific calibration model. In this model it is important to remove interferences such as background from the substrate. Several technical approaches have been developed to meet these challenges for the biological applications of Raman spectroscopy.[13,14]

As shown by our group, many ophthalmic drugs have very specific Raman fingerprint patterns (patterns specific for a drug-molecule).[15] Bauer et al. demonstrated that confocal Raman spectroscopy can be used for pharmacokinetic detection of Dorzolamide ophthalmic solution in tear-film, and corneas of living rabbits.[16] Another study showed the detection of glucose levels in aqueous humor in rabbits [17] and human samples.[18] Sideroudi et al. showed that Raman spectroscopy is of interest to test drug concentrations in an artificial anterior chamber model using ciprofloxacin as target drug.[19] Ganciclovir, ceftazidime and amphotericin B have been detected with Raman spectroscopy in vitro after injecting the drugs into the anterior chamber of rabbit eyes.[13,20] Although these studies demonstrate the potential of Raman spectroscopy, (animal) models are often not representative of the clinical situation. For example, the injection of drugs in the anterior chamber results in far too high drug concentrations in the aqueous humor and a limited distribution through the tissues.[3]

In this study, we designed and performed in vitro and in vivo animal experiments to detect ketorolac tromethamine, a non-steroidal anti-inflammatory drug (NSAID), using Raman spectroscopy and confirm our findings by high-performance liquid chromatography (HPLC).

\subsection{Materials and methods}

\subsubsection{Materials}

Ketorolac trometamine was purchased from MSN laboratories (Telangana, India), ketorolac $0.5 \%$ ophthalmic solution (Acular ${ }^{\mathrm{TM}}$ ) was purchased from Allergan (Dublin, Ireland), Methoce ${ }^{\circledR} 2 \%$ was purchased from OmniVision (Santa Clara, CA, United 
States), and sterile buffered saline solution (BSS), $0.9 \% \mathrm{NaCl}$ solution with a of $\mathrm{pH}$ 7.4 was purchased from B. Braun (Melsungen AG, Germany). Ethylenediaminetetraacetic acid (EDTA) (\#E5134), benzalkonium chloride (BAK) (\#B-1383), methanol (\#34860), and Brand ${ }^{\circledR}$ cuvettes (\#7592-00) were purchased from Sigma-Aldrich (MO, United States). Ketamine was provided by Alfasan (Woerden, the Netherlands), midazolam by Actavis (Dublin, Ireland), and MINIMS ${ }^{\circledR}$ Oxybuprocaine hydrochloride by Bausch \& Lomb Pharma (Brussels, Belgium). Insulin syringes (BD Micro-Fine ${ }^{\mathrm{TM}}$ ) were bought from Becton Dickinson (NJ, United states). MilliQ water and phosphate buffered saline (PBS) ( $\mathrm{pH}$ of 7.4) were freshly produced. Freshly enucleated eyes were kindly provided by "Slachthuis Kerkrade Holding", (Kerkrade, the Netherlands).

\subsubsection{Sample preparation}

Freshly enucleated eyes from the domestic pig (Sus Scrofa Domesticus) were obtained from an abattoir and transported to the laboratory on ice. Before use, the pig eyes were inspected using a stereo microscope (Olympus SZX9, Tokyo, Japan). Only eyes with clear corneas without visible corneal damage were used in the experiment. After removal of excess surrounding tissue, the eyes were washed in PBS. Within 3 hours after enucleation, the pig eyes were submerged in $15 \mathrm{~mL}$ of a dilution of ketorolac tromethamine in PBS in a $50 \mathrm{~mL}$ centrifugal tube. The following ketorolac concentrations were used to submerge the pig eyes: $0.05 \%, 0.1 \%$, $0.125 \%, 0.25 \%, 0.5 \%, 1.0 \%, 1.25 \%, 2.5 \%$, and $5.0 \%$. As negative control, PBS was used, and as positive control Acular ${ }^{\mathrm{TM}}$ was used. Three eyes were used per concentration. The pig eyes were stored for about 24 hours in the dark at $4{ }^{\circ} \mathrm{C}$ and were measured by Raman spectroscopy. After the Raman measurement, $100 \mu \mathrm{L}$ to $150 \mu \mathrm{L}$ aqueous humor was collected using an insulin syringe and the ketorolac concentration was investigated using Raman spectroscopy and HPLC.

\subsubsection{Raman spectroscopy set-up}

Figure 6-1 shows a schematic overview of the used modular confocal Raman spectroscopy system. A diode emitting laser of $785 \mathrm{~nm}$ with a continue power of 26 mW (Innovative Photonic Solutions SM 785 nm, Monmouth Junction, NJ, United States) and a $671 \mathrm{~nm}$ diode emitting laser with a continue power of $14 \mathrm{~mW}$ (Laser Quantum Ignis 671 and SMD 6000, Konstanz, Germany) were used to excite the samples. Raman spectra were recorded with a high-performance Raman module model 2500 (River Diagnostics ${ }^{\circledR}$, Rotterdam, the Netherlands). This module guides the laser light through a diamond optical fiber, shapes and conditions the beam through a pinhole to the measurement stage. First, the light is sent through a collimation lens with a focus length of $80 \mathrm{~mm}$ (f80). In front of the sample, a f80 lens was used when the sample was measured in a cuvette. For the ex vivo pig eye 
experiment, either a long-working-distance microscope objective lens (Jena lens, magnification $\times 25$; numerical aperture $=0.50$; focal length $=10 \mathrm{~mm}$; Carl Zeiss, Jena, Germany), or a lens with a focus length of $60 \mathrm{~mm}$ (f60) in combination with a Gonio lens (Haag-Streit Meridian; CGA1, Köniz, Switzerland) were used. To connect the Gonio lens to the cornea, topically applied Methocel ${ }^{\circledR} 2 \%$ was used. For the in vivo rabbit experiment the f60 lens in combination with a Gonio lens was used. The lens was also connected to the cornea using Methoce ${ }^{\circledR} 2 \%$.

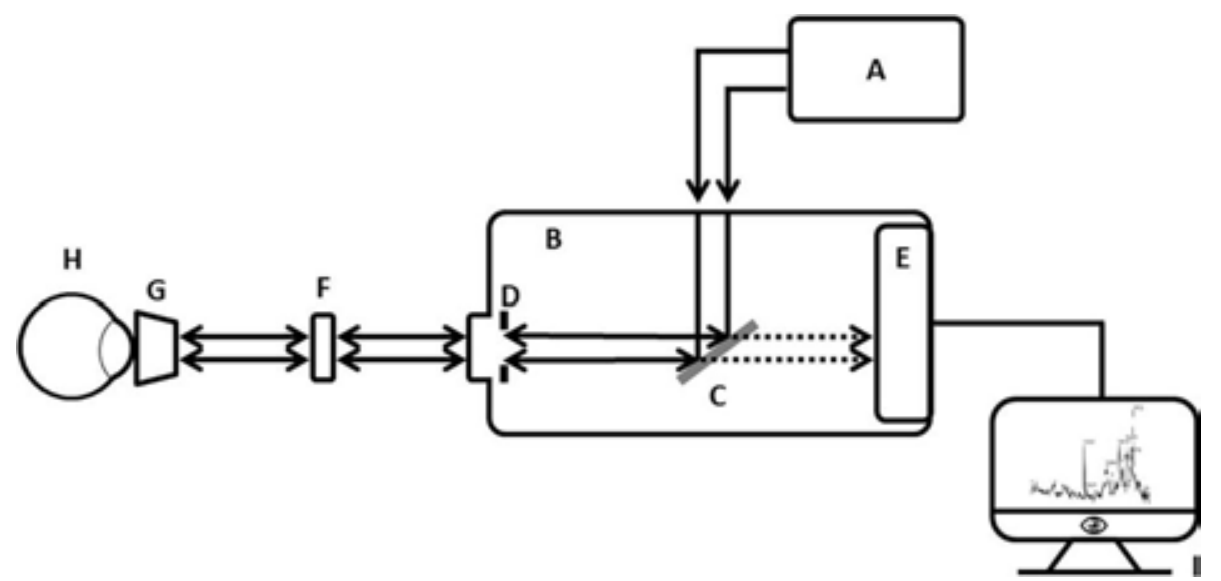

Figure 6-1. Schematic Raman spectroscopy set-up. (A) laser; (B) Raman module, with (C) filter for Raman scattered light; (D) $25 \mu \mathrm{m}$ pinhole; (E) integrated charge-coupled device (CCD); (F) collimation f80 lens; (G) f60 lens with a Gonio (one-mirror) lens, or objective (Jena lens), or a f80 lens; (H) sample; and (I) computer. Arrows indicate direction of (backscattered) laser light; dotted arrows indicate direction of Raman-Scattered light. The Raman spectrometer operates in reflectance mode.

In the experiment the lenses both act to focus the incident light as well as to collect the Raman back-scattered light. As such, the latter is passed back toward the Raman module and projected on a cooled charge-coupled device (CCD) camera (operating temperature $-60^{\circ} \mathrm{C}$ ) for signal detection. Raman back-scattered light in the range of 400 relative wavenumbers $\left(\mathrm{cm}^{-1}\right)$ to $1800 \mathrm{~cm}^{-1}$ was detected using the $785 \mathrm{~nm}$ laser. For the range from $2400 \mathrm{~cm}^{-1}$ to $4000 \mathrm{~cm}^{-1}$ the $671 \mathrm{~nm}$ laser was used. The spectral resolution of the measurements was $2 \mathrm{~cm}^{-1}$ and the samples were exposed to 3 frames counting 60 seconds of exposure during the experiment. The system was used in single point modus (not confocal) and location in the sample was determined using the high wave numbers (671 nm laser).[21] During the in vivo rabbit experiment, the exposure was 2 frames of 30 seconds.

\subsubsection{Identification of ketorolac tromethamine}

Multivariate-peak data analysis takes into account all peaks corresponding to the chemical form ketorolac tromethamine. Elshout et al. published the four most intense 
peaks corresponding to ketorolac tromethamine $(1002 \mathrm{~cm}-1,1524 \mathrm{~cm}-1,1568 \mathrm{~cm}-1$ and $1602 \mathrm{~cm}-1)$.[15] In our set-up, commercially available ketorolac tromethamine eye drops (Acular ${ }^{\mathrm{TM}}$ ) were compared to each individual ingredient to investigate if the additives in Acular ${ }^{\mathrm{TM}}$ interfere with the ketorolac signal in the Raman measurement. Those ingredients were: ketorolac tromethamine (dissolved in PBS, $\mathrm{pH}$ 7.4), EDTA (50 mg/mL dissolved in MilliQ water), BAK (50 mg/mL dissolved in MilliQ water). The anesthetics used in the in vivo experiment were also individually measured using Raman spectroscopy, those compounds were: ketamine, midazolam and Oxybuprocaine hydrochloride. $75 \mu \mathrm{L}$ of each sample was pipetted in a Brand ${ }^{\circledR}$ cuvette and measured using Raman spectroscopy.

\subsubsection{Detection of ketorolac tromethamine in aqueous humor of pig eyes}

An anterior chamber paracentesis of pig eyes was done to collect $100 \mu \mathrm{L}$ to $150 \mu \mathrm{L}$ aqueous humor. After centrifugation (15,000G, 5 minutes at $4^{\circ} \mathrm{C}$ to remove proteins) the supernatant was transferred to a cuvette and measured with Raman spectroscopy.

Hereafter, the samples were fivefold diluted using methanol, centrifuged once more $\left(15,000 \mathrm{G}\right.$ for 5 minutes at $\left.4^{\circ} \mathrm{C}\right)$ where after the supernatant was analyzed by HPLC (Agilent 1260 infinity series with EZchrom software, Agilent inc. Santa Clara, CA, United States). Analysis was done according to the US Pharmacopeia [22], using an elution time of 20 minutes and injection volume of $10 \mu \mathrm{L}$, and peak UV-detection at

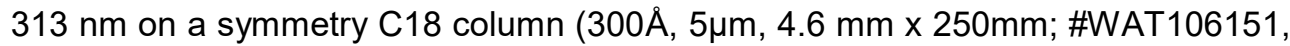
Waters corp., Milford, MA, United States) with a symmetry C8 VanGuard pre-column

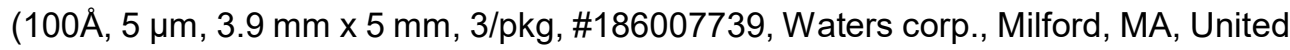
States). Ketorolac had a retention time of 10.5 minutes, has a limit of detection (LOD) of $4 \mathrm{ng} / \mathrm{mL}$, and a limit of quantification (LOQ) of $10 \mathrm{ng} / \mathrm{mL}$.

\subsubsection{In vivo detection of ketorolac tromethamine in rabbits}

All animal procedures were conducted according to the ARVO Statement for the Use of Animals in Ophthalmic and Visual Research and the Guidelines of the Central Laboratory Animal Facility of Maastricht University. All protocols were approved by the Central Committee for Animal research and were in accordance with the European Guidelines (2010/63/EU).

Twelve New-Zealand white rabbits (weight between $2.0 \mathrm{~kg}$ and $2.5 \mathrm{~kg}$ upon arrival) were ordered from Envigo (Horst, NL) and housed in group housing, 6 animals per cage, males and females separated. The rabbits had ad libitum access to water and received $100 \mathrm{gr}$. rabbit chow per animal per day. Before the animals were used in experiment, they had one week to acclimatize. The rabbits were treated with $50 \mu \mathrm{L}$ 
$0.5 \%$ ketorolac tromethamine ophthalmic solution (Acular ${ }^{\mathrm{TM}}$ ) in the lower conjunctival fornix. The contralateral eye was treated with $50 \mu \mathrm{L}$ BSS. Both treatments were performed three times a day for a total of 28 days, equivalent to a clinically used drop regime.

On day 0 , day 7 , day 14 , day 21 , and day 28 the rabbits were measured. All measurements were performed 1 to 3 hours after receiving the eye drops. During the examinations, rabbits were anesthetized with ketamine $(50 \mathrm{mg} / \mathrm{kg})$ and midazolam $(5 \mathrm{mg} / \mathrm{kg})$ intramuscularly. First, a Raman measurement was performed on both eyes, followed by an anterior chamber paracentesis (drawing $50 \mu \mathrm{L}$ ) of the right eye. Before the paracentesis, the eye received additional topical sedation using one drop of $0.4 \%$ Oxybuprocaine hydrochloride solution. Aqueous humor samples were frozen on dry ice immediately after sampling and stored in a $-80^{\circ} \mathrm{C}$ freezer until further processing. As a negative control, aqueous humor was drawn $(100 \mu \mathrm{L})$ from both eyes of seven healthy control animals, after sacrifice. The negative control animals did not received any topical treatment nor anesthetics. Aqueous humor was drawn within 10 minutes after sacrificing of the rabbits. A total of thirteen samples was collected (one sample was lost during processing).

Aqueous humor samples were measured with the Raman spectrometer followed by HPLC analysis using protocols as described earlier.

\subsubsection{Pre-processing of the raw acquired Raman spectrum}

In order to extract Raman signal from the raw acquired spectrum, it is necessary to pre-process the acquired spectrum.[23] Cosmic ray spikes, randomly generated due to cosmic radiation, were replaced by the average intensity from the neighboring frames. A partial polynomial fitting method combined with the morphology approach of Perez-Pueyo et al. [24] was used to remove instrumental noise. First, spectra were dissected in different zones. Zones that only contain background fluorescence were used to calculate the polynomial function coefficients. The zone that contains the water-peak $\left(1550 \mathrm{~cm}^{-1}\right.$ to $\left.1650 \mathrm{~cm}^{-1}\right)$ was excluded from the fitting calculation. The achieved polynomial function was applied on the full spectrum $\left(400 \mathrm{~cm}^{-1}\right.$ to $1700 \mathrm{~cm}^{-}$

$\left.{ }^{1}\right)$ to remove the fluorescence background. Hereafter, the morphology-based approach was applied to eliminate instrumental noise.

In short, our pre-processing procedure are as follows: first, manual cosmic ray removal before any further treatment (figure 6-S1,1). Second, averaging of the frames to minimize the fluctuations. Third, applying partial polynomial $\left(5^{\text {th }}\right.$ degree) fitting on the averaged spectrum for subtraction of fluorescence (figure 6-S1,3) and fourth, using morphology method to eliminate the instrumental noise (figure 6-S1,2). Besides the first step, all procedures are processed by a self-developed MATLAB program (Version 2017b, The Mathworks Inc., Natick, MA, United States). 
Furthermore, all samples were normalized by dividing ketorolac related peaks by their water-peak $\left(1642 \mathrm{~cm}^{-1}\right)$ [25] correcting for the sample-sample variation.

\subsubsection{Statistical analysis}

Statistical analysis was performed using GraphPad Prism version 6.01 (GraphPad Software inc. La Jolla, CA, United States). All in vitro pig eye data were analyzed using 2-way ANOVA multiple comparison tests. The in vivo rabbit data was analyzed with paired t-tests. The in vitro rabbit data was analyzed using unpaired t-tests because the aqueous humor samples came from different rabbits. All data are shown as mean \pm SD.

\subsection{Results}

\subsubsection{Detection of peaks related to ketorolac tromethamine using Raman spectroscopy}

Figure 6-2 shows the Raman spectrum of Acular $^{\mathrm{TM}}$ and PBS. For the multivariate peak analysis, eight high-intensity peaks specific for ketorolac tromethamine were selected: $1002 \mathrm{~cm}^{-1}, 1282 \mathrm{~cm}^{-1}, 1348 \mathrm{~cm}^{-1}, 1432 \mathrm{~cm}^{-1}, 1472 \mathrm{~cm}^{-1}, 1524 \mathrm{~cm}^{-1}, 1568$ $\mathrm{cm}^{-1}$, and $1602 \mathrm{~cm}^{-1}$ as shown in figure 6-2 (upper spectrum). These peaks were not related to additives such as EDTA or BAK (figure 6-S2). The $1602 \mathrm{~cm}^{-1}$ peak overlaps partially with the water-peak (1642 $\mathrm{cm}^{-1}$, underlined in both spectra), this peak is not used during further processing. In the following results, the intensity of each individual peak or the average intensity ratio of the seven peaks is plotted and used. Four background peaks originating from PBS, aqueous humor and cuvette were detected: $930 \mathrm{~cm}^{-1}, 1120 \mathrm{~cm}^{-1}, 1448 \mathrm{~cm}^{-1}$, and $1642 \mathrm{~cm}^{-1}$ (figure 6-2 lower spectrum). These four peaks were identical in PBS and AH (figure 6-S4). No peak differences were found between aqueous humor from rabbits and pigs (data not shown).

\subsubsection{Detection of ketorolac tromethamine using the HPLC in aqueous humor of pig eyes}

Figure 6-3a shows the HPLC quantification of ketorolac tromethamine (concentration curve $0.05 \%$ to $1.25 \%$ ), and the ketorolac concentrations in the aqueous humor of pig eyes that were immersed in a similar concentration range as described earlier. Both solutions demonstrate linearity with the tested concentrations, $R^{2}$ of 0.97 and $\mathrm{R}^{2}$ of 0.88 respectively. When comparing the ketorolac concentration in Acular ${ }^{\mathrm{TM}}$ to the aqueous humor penetrated ketorolac concentration in pig eyes, after submerging for 24 hours in Acular $^{\mathrm{TM}}$, approximately one fourth of the original ketorolac concentration appeared to have penetrated into the aqueous humor. The concentration in the aqueous humor of the pig eye was significantly lower $(p=$ 
0.0012) compared to the concentration in which the eye was submerged (figure 6$3 b)$.

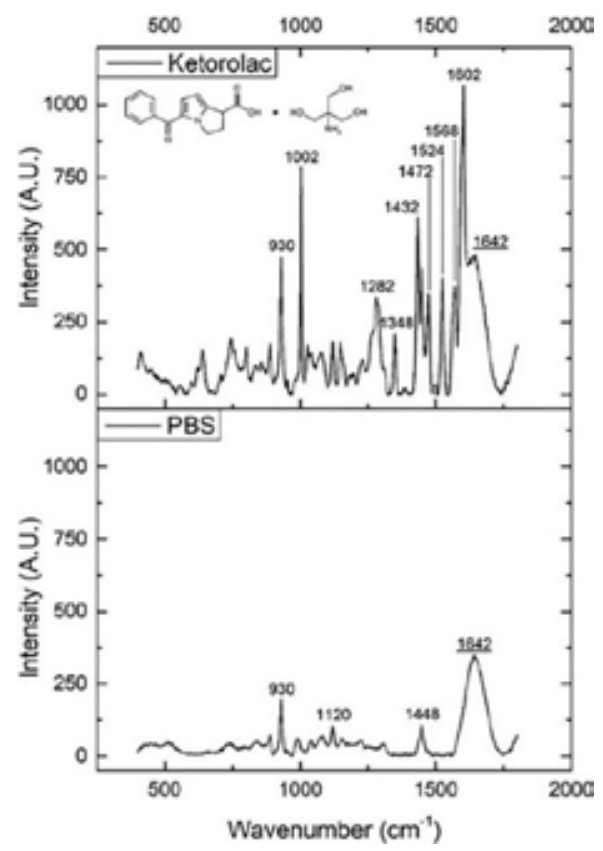

Figure 6-2. Raman fingerprint spectra of Acular ${ }^{\mathrm{TM}}$ $(0.5 \%$ ketorolac tromethamine ophthalmic solution) and PBS. The structural formula of ketorolac tromethamine is shown in the frame related to Acular ${ }^{\mathrm{TM}}$. The numbers above the peak represent the corresponding wavenumbers. Baseline correction is applied using polynomial correction. PBS $=$ phosphate buffered saline, A.U. = arbitrary unit.
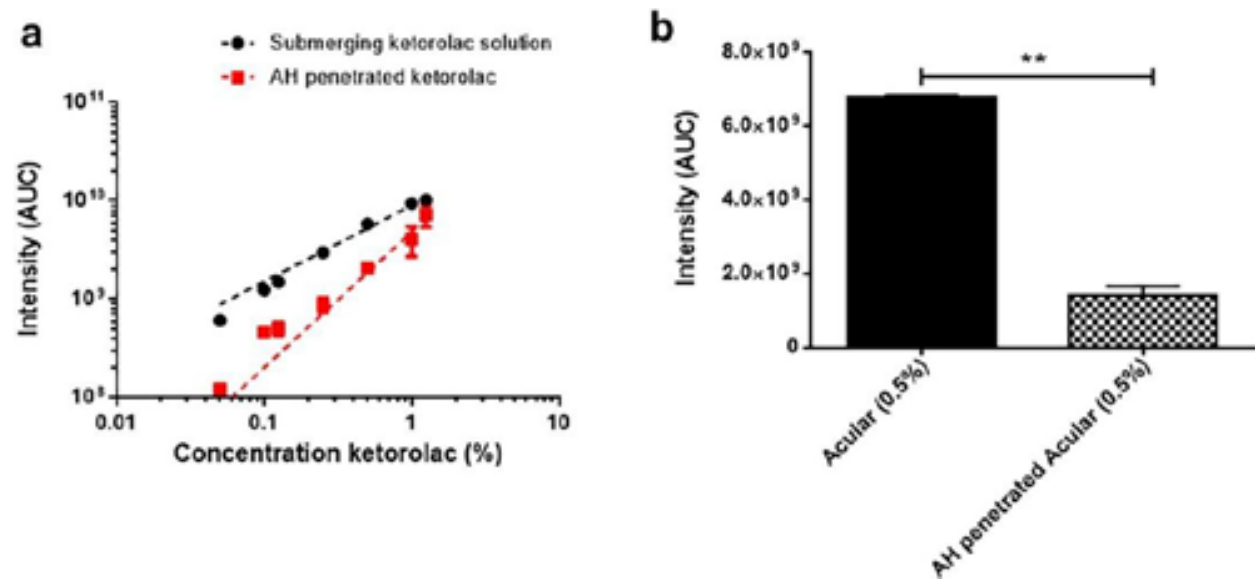

Figure 6-3. HPLC detection and analysis of (a) a dilution curve of ketorolac tromethamine in PBS (submersion solution) (black) and the detection of penetrated ketorolac in aqueous humor (red). The dashed line represents a logarithmic trendline with a R2 of 0.98 and 0.89 for the black and red points respectively. (b) HPLC analysis of ketorolac tromethamine in Acular ${ }^{\mathrm{TM}}$ and the penetrated concentration in aqueous humor after $24 \mathrm{~h}$. Compared using students t-test, ${ }^{* *} \mathrm{p}=0.0012, \mathrm{n}=3, \mathrm{AH}=$ aqueous humor, $\mathrm{AUC}=$ area under the curve, data is plotted as mean $\pm \mathrm{SD}$. 


\subsubsection{Detection of ketorolac tromethamine using Raman spectroscopy in pig eyes}

Figure 6-4a provides a detailed overview of the intensity ratios corresponding to ketorolac peaks of Acular ${ }^{\mathrm{TM}}$ compared to PBS. All peaks related to ketorolac are about five to one hundred and fifty times higher in Acular ${ }^{\mathrm{TM}}$ compared to PBS. After penetration in the aqueous humor of pig eyes, the same ketorolac peaks could be detected, as shown in figure 6-4b. However, no significant difference between the ketorolac and the PBS peak on wavenumber $1348 \mathrm{~cm}^{-1}$ was observed.

When the intensity of the peaks is summarized, the aqueous humor penetrated concentration of ketorolac is about one fourth of the original solution (figure 6-4c). As expected, there is no difference between PBS and the aqueous humor penetrated PBS signal. These results demonstrate that Raman spectroscopy is able to detect ketorolac solutions after penetration in the aqueous humor.

Figure 6-5 shows a correlation between the Raman signal and HPLC signal from the concentration curve of aqueous humor penetrated ketorolac in pig eyes. This resulted in Pearson's coefficient of 0.89 with a $\mathrm{R}^{2}$ of 0.79 using correlation on the log-log scale.

Figure 6-6 demonstrates the quantitative potential of confocal Raman spectroscopy. Figure 6-6a shows a linear relationship between the concentration and the observed signal for three different set-ups. The limit of detection of the ketorolac dilution curve $(0.05 \%$ to $5.0 \%$ )(black line) lies on an intensity ratio of $0.05 \pm 0.003$, which is lower than the background signal of aqueous humor detected in a cuvette (red line) $(0.07$ \pm 0.02 ). The limit of detection of aqueous humor detected with a Jena lens (green line) or a Gonio lens (blue line) lies however, about nine times higher $(0.45 \pm 0.03$ and $0.34 \pm 0.09$, respectively).

Figure 6-6b shows the response of the Raman system with three different set-ups. Different intensity ratios when comparing Acular ${ }^{\mathrm{TM}}$ to PBS have been observed. When measuring Acular ${ }^{\mathrm{TM}}$ and PBS in a cuvette, a clear difference is visible with low background. When the ketorolac concentration in aqueous humor of pig eyes is measured in a cuvette, the intensity ratio of Acular ${ }^{\mathrm{TM}}$ is lower, but the background has slightly increased. Aqueous humor in the anterior chamber, measured using the Jena lens does not show a difference between an Acular ${ }^{\mathrm{TM}}$ submerged pig eye and a PBS treated pig eye due to high background noise. When ketorolac is detected in aqueous humor in the anterior chamber using the Gonio lens, a high background is observed; however, the Gonio lens is capable to distinguish ketorolac from the PBS samples. 
a
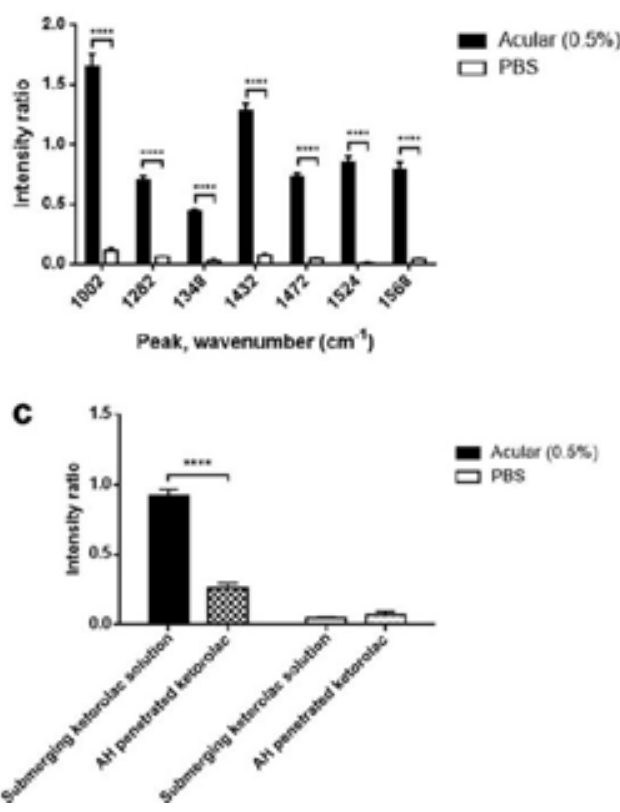

b

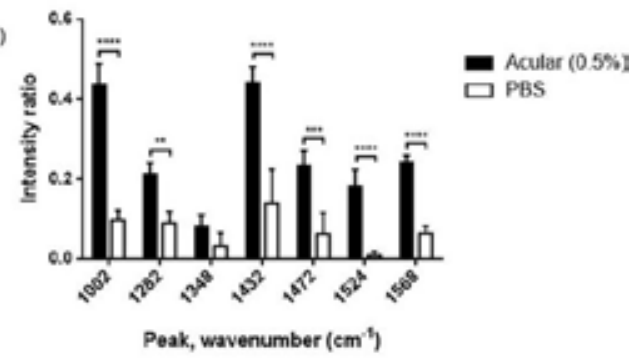

Figure 6-4. Raman detection of ketorolac in aqueous humor of pig eyes. (a) Peaks corresponding to Acular ${ }^{\mathrm{TM}}$ compared to PBS and (b) after penetration in aqueous humor of pig eyes $(n=3)$, row comparison using Tukey's multiple comparisons test. (c) Averaged intensity ratios from Acular ${ }^{\mathrm{TM}}$ and PBS as the submerging solution and after aqueous humor penetration in pig eyes. Exposure time $60 \mathrm{~s}, 3$ frames averaged, $785 \mathrm{~nm}$ laser. Peaks normalized by dividing the intensity of each peak with the intensity of the peak at $1,642 \mathrm{~cm}^{-1}, \mathrm{n}=3 .{ }^{* *}=\mathrm{p}<0.01,{ }^{* * *}=\mathrm{P}<0.001,{ }^{* * * *}=\mathrm{P}<0.0001$. AH, aqueous humor, data is plotted as mean $\pm \mathrm{SD}$.

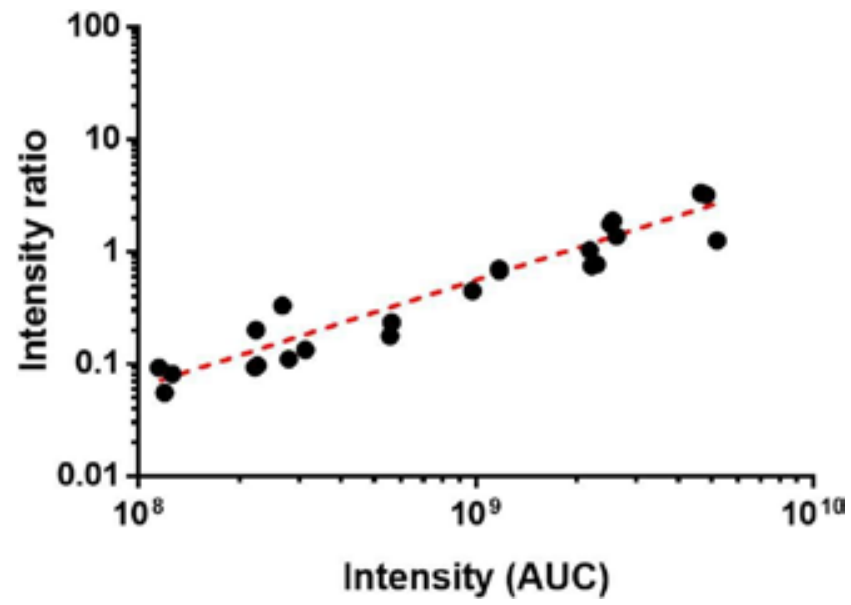

Figure 6-5. Correlation between Raman signal (y-axis) and the HPLC signal (x-axis) of aqueous humor from pig eyes submerged in ketorolac dilutions. The dashed line represents a logarithmic trendline. Pearson's $r$ of 0.89 and a $R^{2}$ of 0.79 , every dot represents one eye $(n=3$ per concentration). AUC $=$ area under the curve. 

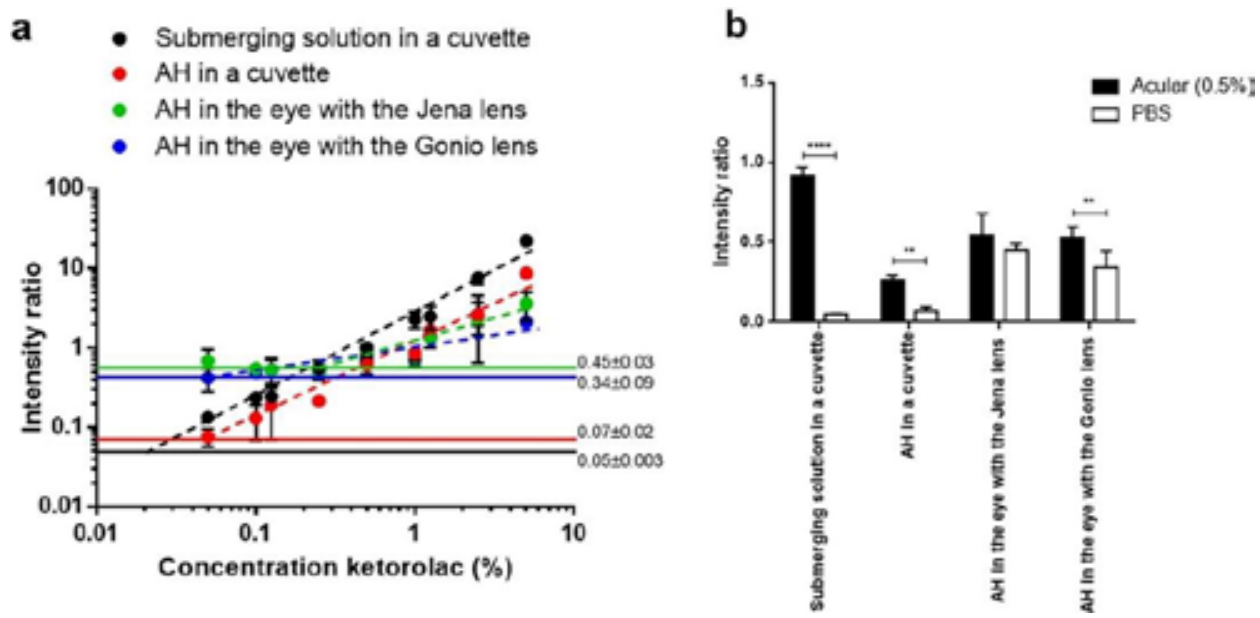

Figure 6-6. In vitro detection of ketorolac in pig eyes using three different set-ups (aqueous humor in a cuvette, aqueous humor in the anterior chamber with the Jena lens and aqueous humor in the anterior chamber with the Gonio lens) and the control situation (submerging fluid in a cuvette). (a) Dilution series of ketorolac $(0.05 \%$ to $5.0 \%)$ were measured with the Raman spectrometer. Black dots represent the ketorolac tromethamine dilution measured in a cuvette. Red dots represent aqueous humor of pig eyes submerged in corresponding ketorolac tromethamine dilutions after paracentesis measured in a cuvette. The green dots represent measurements on the pig eye, in the anterior chamber with the Jena lens, and the blue dots represent measurement on the eye into the anterior chamber with the Gonio lens. The dashed-lines are drawn as guide to the eyes whereas the solid lines provide the limit of detection. (b) Bar graph with the response of the detection of Acular ${ }^{\mathrm{TM}}$ and PBS. The fluids have been measured in a cuvette, in the aqueous humor using a cuvette, or in the eye with the Jena lens, or in the eye with a Gonio lens. Samples are compared using ANOVA multiple comparison tests, ${ }^{*} \mathrm{P}<0.05$, ${ }^{* *} \mathrm{P}<0.01,{ }^{* * *} \mathrm{P}<0.001$ and ${ }^{* * *} p<0.0001, n=3$ per point. $A H=$ aqueous humor, $A C=$ anterior chamber, data is plotted as mean $\pm S D$.

\subsubsection{In vivo detection of ketorolac in rabbit eyes}

Figure 6-7a shows that the Gonio lens was able to measure aqueous humor in the anterior chamber of living rabbits; however, no difference between treated (Acular ${ }^{\mathrm{TM}}$ ) and non-treated (BSS) rabbit eyes was observed. When the Gonio lens was not properly aligned on the eye, the lens, or the cornea was measured, as shown in figure $6-7 b$ and figure $6-7 c$ respectively. In none of the in vivo measurements a significant difference between the treated eye (OD) and the control eye (OS) was observed.

The Raman spectra show the wavenumber $(671 \mathrm{~nm})$ signals corresponding to the location in the eye. When measuring specifically in the aqueous humor, a broad peak is visible between $3000 \mathrm{~cm}^{-1}$ and $3700 \mathrm{~cm}^{-1}$ (figure 6-7a, lowest frame). The lens shows a narrow peak at $2900 \mathrm{~cm}^{-1}$ and a broad one between $3000 \mathrm{~cm}^{-1}$ and 3700 $\mathrm{cm}^{-1}$ (similarly to the peak for aqueous humor) (figure 6-7b lowest frame). The cornea expresses a high peak at $2900 \mathrm{~cm}^{-1}$ and a broad peak between $3000 \mathrm{~cm}^{-1}$ and 3700 $\mathrm{cm}^{-1}$ (figure 6-7c lowest frame). 
a
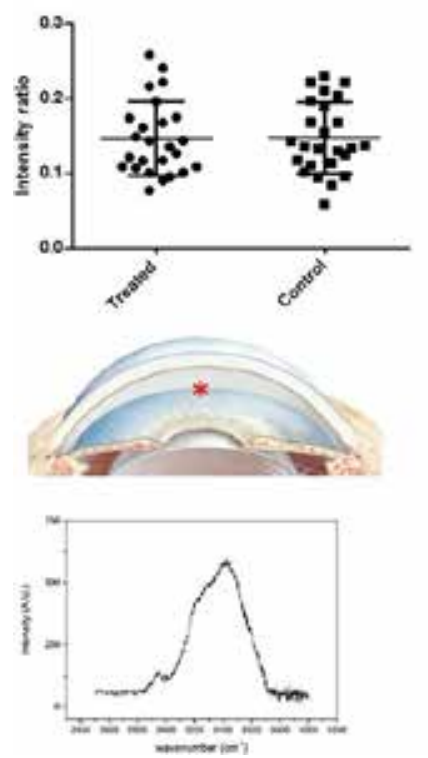

b
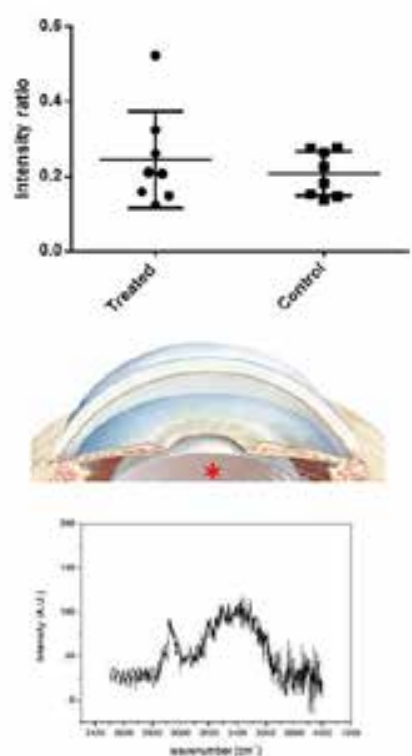

c
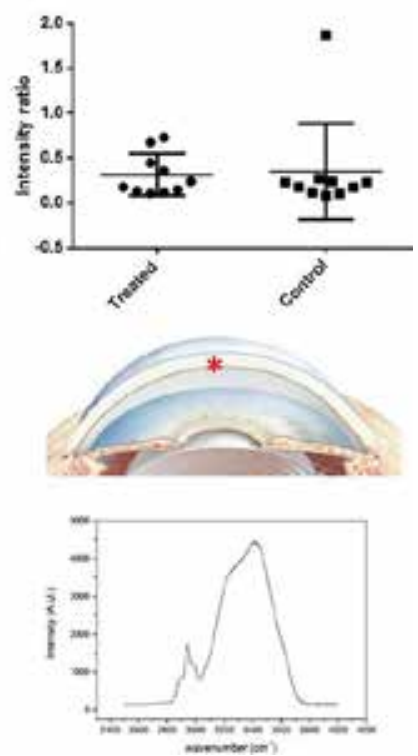

Figure 6-7. In vivo detection of ketorolac in rabbit eyes. (a) Detection in aqueous humor when the Gonio lens is properly aligned on the eye. The asterisk shows the location of focus and the Raman spectrum provides the high wavenumber $(671 \mathrm{~nm})$ measurement to assure correct location in the eye. (b) Measurement on the lens or (c) on the cornea, due to misalignment of the Gonio lens. Treated eye received three times a day $50 \mu \mathrm{L}$ Acular ${ }^{\mathrm{TM}}$, and the control eye was treated with $50 \mu \mathrm{L}$ BSS $(\mathrm{pH} 7.4)$. Paired tests have been executed to test difference between treated and control eyes. Each dot represents one measurement at one rabbit, data is plotted as mean \pm SD.

\subsubsection{Ketorolac tromethamine detection in aqueous humor of rabbits ex vivo}

Figure 6-8 shows the ex vivo detection of the ketorolac concentration in aqueous humor from rabbits, measured in a cuvette by Raman and via HPLC. As shown in figure 6-8a, a significant difference $(p=0.0017)$ was observed between Acular ${ }^{\mathrm{TM}}$ treated eyes and non-treated eyes when the ketorolac was measured using Raman spectroscopy. However, due to the large inter-sample variation, no exact concentration could be calculated and linked to individual measured aqueous humor samples measured by our Raman spectroscopic system.

Figure 6-8b shows the detection of the concentration ketorolac from aqueous humor of rabbits using HPLC. These results also show significant difference $(p<0.0001)$ between the treated and untreated eyes. When all days are combined, the average drug concentration was $927 \mathrm{ng} / \mathrm{mL} \pm 430 \mathrm{ng} / \mathrm{mL}$ (mean $\pm \mathrm{SD}$ ), a maximum detected concentration of $2236 \mathrm{ng} / \mathrm{mL}$ and a minimum detected concentration of $63 \mathrm{ng} / \mathrm{mL}$ in the treated eyes. The control eyes did not show any signal above noise level when 
detected with HPLC. No clear correlation between the Raman signal and HPLC concentration could be found with these low concentrations.

a

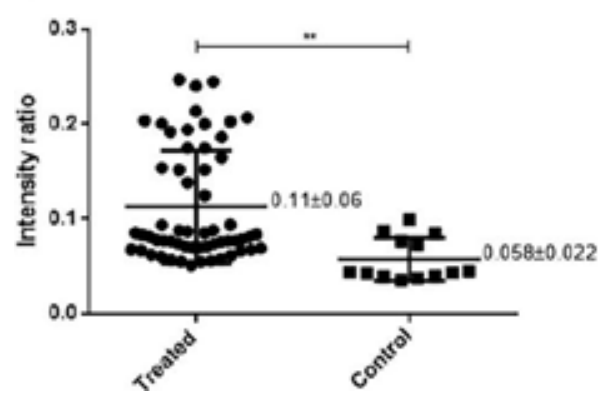

b

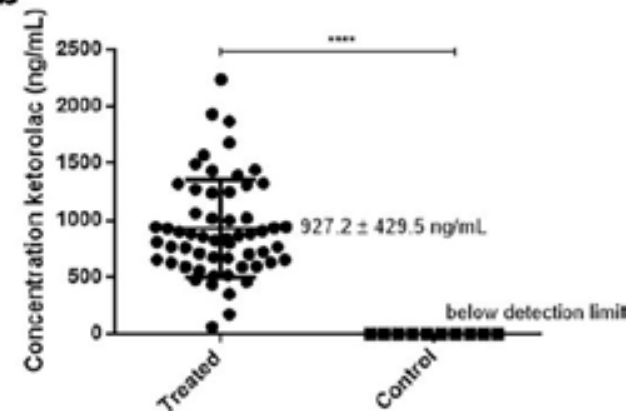

Figure 6-8. Ex vivo detection of ketorolac tromethamine in aqueous humor from rabbit eyes. General grouped difference between the aqueous humor of treated eyes and control (non-treated) eyes measured with (a) Raman spectroscopy and (b) HPLC. ${ }^{* *} p<0.01$, ${ }^{* * * *} p<0.0001$. Each dot represents one sample from one rabbit, data tested using student t-test and is plotted as mean $\pm S D, n_{\text {treated }}=58$ and $n_{\text {control }}=13$.

\subsection{Discussion}

The aim of the study was to evaluate the quantitative use of Raman spectroscopy for the in vivo detection of drug levels in the anterior chamber of the eye. Firstly, we needed to optimize the method to analyze our Raman data. The probability of Raman scattering is much lower than intrinsic fluorescence emission in biological samples. Therefore, in order to extract Raman signal from the raw acquired spectrum, it was necessary to pre-process the acquired spectrum.[23] Furthermore, cosmic ray spikes, randomly generated due to cosmic radiation, which affected different wavenumbers each time, needed to be removed.[23,26] For the latter, there are two approaches described in the literature.[26,27] In this study, we went for the easiest method, by replacing the intensities of the cosmic peaks with the average from the neighboring frames (left and right from the ray) as suggested by Zhang et al.[26] Several approaches have been proposed to minimize the influence from the background fluorescence.[13] The most accepted method for background subtraction is polynomial fitting but as mentioned by Byrne et al. no standardized protocols are available.[13] Zhao et al. introduced an automated polynomial background subtraction method for biomedical applications, which could subtract the background fluorescence.[28] Zhang et al. developed an automated method for fluorescence background subtraction named "automatic pre-processing method for Raman imaging data set (APRI)".[26] However, previous methods encountered difficulties when handling spectrums containing instrumental noise. In some in vivo experiments, the contribution from instrumental noise is inevitable and cannot be neglected, thus affecting the conventional polynomial methods. Hence, further 
treatments have been developed to eliminate the instrumental noise. Perez-Pueyo et al. introduced a morphology-based baseline removal method for Raman spectrums.[24] It employs Tophat filtering using basic operations as dilation and erosion to filter the features beyond or below a pre-set threshold, thereby removing the instrumental noise. In this study, we used a partial polynomial fitting method combined with the morphology approach of Perez-Pueyo et al. to remove instrumental noise.[24] Hereafter we normalized all samples by dividing ketorolac related peaks by their water-peak $\left(1642 \mathrm{~cm}^{-1}\right)$ [25], thereby correcting for the samplesample variation. Overall, we created a solid method to remove hardware related shifts from Raman data.

After we optimized the analysis for Raman spectroscopy, we first confirmed the four ketorolac tromethamine peaks described by Elshout et al. [15] and then identified four additional peaks specific for ketorolac. We observed that the $1602 \mathrm{~cm}^{-1}$ peak partially overlaps with the water-peak $\left(1642 \mathrm{~cm}^{-1}\right)$; therefore, this peak was ignored during processing.

During the experiment, we examined buffered saline solutions (PBS and BSS), aqueous humor from pig eyes and aqueous humor from rabbit eyes. Although, there are differences between the buffered solutions and aqueous humor, the Raman signal was identical, as shown in supplementary figure 6-S4, and also did not affect the HPLC results. In the pig model, a concentration range of dissolved ketorolac was used and Acular ${ }^{\mathrm{TM}}$ was used as a positive control, whereas only Acular ${ }^{\mathrm{TM}}$ was used in the rabbit model. No differences were found in Raman signal, nor have we observed shifted peaks during the experiments. Although we expected that the Raman signals might be affected due to the additives, we did not observe any interference of the additives on the ketorolac signal (figure 6-S2). Neither have we observed influences of the anesthetics on the Raman signal in the in vivo experiment (figure 6-S5). However, we observed superior penetration of dissolved ketorolac in PBS compared to the commercially available solution (Acular ${ }^{\mathrm{TM}}$ ) (figure 6-S6). The $\mathrm{pH}$ of both solutions was similar ( $\mathrm{pH} 7.4$ ) but the osmolality differs, i.e. Acular ${ }^{\mathrm{TM}}$ displays an osmolality of $290 \mathrm{mOsmol} / \mathrm{kg}$ [29] whereas dissolved ketorolac tromethamine in PBS has a (theoretical) osmolality of $330 \mathrm{mOsmol} / \mathrm{kg}$. This could explain the higher ocular penetration by the dissolved ketorolac solution. Lee et al. also found osmolality to be a relatively large influencer of ocular penetration in studying penetration of topically applied Atenolol.[30] However, in our experiment we used post mortem tissue in which the cellular membranes, and tight junctions between the cells are affected and in which clearance of the drug is hampered. Due to submerging of completely enucleated eyes more scleral diffusion is expected leading to increased intraocular drug concentrations as compared to eye drops.[31,32] Furthermore, the long contact time of 24 hours also enhanced the intraocular drug concentration. The detection of ketorolac in the in vitro model was 
also challenging due to corneal haze in the stromal layers after leaving the eyes in the buffered solutions (figure 6-S3), high background signals were detected which disturbed the Raman measurements. This resulted in high background noise signals that were more than three times higher (when normalized), compared to regular cuvette measurements (figure 6-6b).

The in vivo animal model represents a realistic clinical situation of an eye drop scheme. The detection of the ketorolac concentration is in line with previously published data. Ling et al. found a $\mathrm{C}_{\max }$ of $1905 \mathrm{ng} / \mathrm{mL}$.[33] Although we were not looking for a $\mathrm{C}_{\max }$, our highest detected concentration was $2236 \mathrm{ng} / \mathrm{mL}$. The mean ketorolac concentration in our experiment was $927 \mathrm{ng} / \mathrm{mL} \pm 430 \mathrm{ng} / \mathrm{mL}$, whereas an average concentration of $1079 \mathrm{ng} / \mathrm{mL} \pm 882 \mathrm{ng} / \mathrm{mL}$ is found in human eyes, when instilling Acular ${ }^{\mathrm{TM}}$ eye drops four times a day, two days pre-surgery.[34]

In our Raman system, two different lenses were compared: a Jena lens and a Gonio lens. Since we used a f60 lens in front of the Gonio lens (the Gonio lens itself does not provide any focus power), better focus was achieved compared to use of the Jena lens. The $f 60$ lens has a smaller numerical aperture, which provides a longer integration length. Second, based on the safety point of view, the Gonio lens prevent the laser from direct illumination on the retina, preventing it for the possible light damage. The excitation laser directly illuminates the retina in the Jena lens setup, it limits the common performance improvement methods like raising the laser power or increasing integration time. Besides, we observed a specific drug related difference when we used a Gonio lens. As such, we continued the experiment in vivo only with the Gonio lens. In vivo the average background signal in the aqueous humor was lower $(0.15 \pm 0.05)$ (figure 6-7a, control eye) compared to in vitro signal $(0.34 \pm 0.09$, Gonio lens, PBS) (figure 6-6). Hence, we tend to conclude that the corneal haze was affecting the signal. However, the drug concentration in the aqueous humor was too low to detect using in vivo Raman spectroscopy. Another important factor affecting the sensitivity of Raman spectroscopy is the exposure time during the measurement. All in vitro samples have been exposed 60 seconds for 3 frames, whereas the in vivo exposure was 30 seconds with 2 frames. We lowered the exposure time and the number of frames in vivo to assure safety of the technique in the rabbits. Because of the limited number of frames the threshold of the intensity ratio $(0.15 \pm 0.05)$ was higher in vivo (figure $6-7 a$, control eye) compared to in vitro intensity ratio $(0.11 \pm 0.06)$ (figure $6-8 a$, control eye). Besides the lower number of frames, also a shorter exposure time may lead to a decreased Raman signal.[35] Due to the large inter-measurement-variations, the standard deviation in the Raman experiment was too large to clearly quantify the in vivo samples. The variation with the HPLC was much smaller resulting in a detection accuracy of nanograms per milliliter. Due to large variations in the in vivo fingerprint signals, no correlation could be found using the rabbit samples, whereas there is a clear correlation with Raman 
spectroscopy and HPLC when measuring higher drug concentrations from the in vitro pig eyes (figure 6-7). Overall, multiple factors affect the readout and it is difficult to select one parameter causing low sensitivity with Raman spectroscopy. In vitro samples have been centrifuged to remove proteins, which could be a reason for higher signal during the in vitro measurements compared to the in vivo measurements. Furthermore, the cornea consists of a $500 \mu \mathrm{m}$ thick stroma, which could scatter or absorb Raman scatter on its way through. Our experiment also shows that the conditions of the cornea could affect the Raman signal sensitivity (corneal haze). Finally, the temperature might also slightly influence the Raman intensity both for the target components and backgrounds. The cuvette samples and in vitro samples were tested at room temperature (about $22^{\circ} \mathrm{C}$ ) while the temperature of in vivo measurement is around $35^{\circ} \mathrm{C}$ in a rabbit eye. It is noticeable that all factors together lower the sensitivity of Raman spectroscopy.

To increase sensitivity in a Raman system, laser power could be increased. The problem however, with increasing laser power in the eye, is the irreversible damage of the photosensitive layers that could occur. In order to protect the eyes, we used a laser power of $26 \mathrm{~mW}$, which is relatively low compared to laser powers which are used on skin $(80 \mathrm{~mW})$ [36], or on cells or tissue sections $(60 \mathrm{~mW})$.[37] For in vitro detection of corneal biomarkers an intensity of $300 \mathrm{~mW}$ is used [38], and even $1 \mathrm{~W}$ is used to create virtual cross-sections of intact eye tissue without dependence on tissue processing.[39] According to Marro et al., laser powers up to $100 \mathrm{~mW}$ are safe to use on retina organotypic cultures (in vitro).[40] Besides laser power, the wavelength and the exposure time are of importance for the prevention of tissue damage. In our study, we used ketorolac tromethamine. Other ocular drugs, however, may have a stronger Raman signal and can be easier to detect in the anterior chamber. All these parameters make Raman spectroscopy a challenging technique. Furthermore, as mentioned by Byrne et al. [13], there is no common accepted manner to correct Raman data. Due to hardware influences and sampleto-sample variation, every Raman spectrometer needs its own corrections.

\subsection{Conclusion}

In this study, we show the value of Raman spectroscopy for the detection of drugs in the anterior chamber of the eye. As expected, the sensitivity and the limit of detection of the HPLC are much higher compared to Raman spectroscopy. However, Raman spectroscopy shows unique potential as a non-invasive technique for real time biomedical analysis. We found good correlation between Raman spectroscopy and HPLC for in vitro detection of drugs. Unfortunately, our Raman spectroscopic system is not yet able to detect a clinically relevant dose of ketorolac tromethamine in the anterior chamber of rabbits in vivo. More research should be conducted to 
increase the sensitivity of Raman spectroscopy while still using low, non-damaging, laser powers.

\subsection{References}

1.

2. 163.

\section{3.}

4.

5. Hornof, M., Toropainen, E., and Urtti, A., Cell culture models of the ocular barriers. Eur J Pharm Biopharm, 2005. 60(2): p. 207-225.

6. Raman, C.V. and Krishnan, K.S., A New Type of Secondary Radiation. Nature, 1928. 121: p. 501-502.

7. Bauer, N.J.C., Potential applications of confocal Raman spectroscopy in ophthalmology, in Ophthalmology. 1999, Datawyse: Maastricht. p. 160.

8. Erckens, R.J., Development of the Raman spectroscopic technique for in vivo applications in the eye, in Ophthalmology. 2001, Unigraphic: Maastricht. p. 140.

9. Gowen, A.A., Marini, F., Tsuchisaka, Y., De Luca, S., Bevilacqua, M., O'Donnell, C., Downey, G., and Tsenkova, R., On the feasibility of near infrared spectroscopy to detect contaminants in water using single salt solutions as model systems. Talanta, 2015. 131: p. 609-618.

10. Li, B., Ryan, P.W., Ray, B.H., Leister, K.J., Sirimuthu, N.M., and Ryder, A.G., Rapid characterization and quality control of complex cell culture media solutions using raman spectroscopy and chemometrics. Biotechnol Bioeng, 2010. 107(2): p. 290-301.

11. Long, D.A., Handbook of Raman spectroscopy. From the research laboratory to the process line, in Journal of Raman Spectroscopy, I.R.L.a.H.G.M. Edwards., Editor. 2004, Marcel Dekker: New York and Basel. p. 91-91.

12. Yun, S.H. and Kwok, S.J.J., Light in diagnosis, therapy and surgery. Nat Biomed Eng, 2017. 1.

13. Byrne, H.J., Knief, P., Keating, M.E., and Bonnier, F., Spectral pre and post processing for infrared and Raman spectroscopy of biological tissues and cells. Chem Soc Rev, 2016. 45(7): p. $1865-1878$.

14. Willemse-Erix, D.F., Scholtes-Timmerman, M.J., Jachtenberg, J.W., van Leeuwen, W.B., Horst-Kreft, D., Bakker Schut, T.C., Deurenberg, R.H., Puppels, G.J., van Belkum, A., Vos, M.C., and Maquelin, K., Optical fingerprinting in bacterial epidemiology: Raman spectroscopy as a real-time typing method. J Clin Microbiol, 2009. 47(3): p. 652-659.

15. Elshout, M., Erckens, R.J., Webers, C.A., Beckers, H.J., Berendschot, T.T., de Brabander, J., Hendrikse, F., and Schouten, J.S., Detection of Raman spectra in ocular drugs for potential in vivo application of Raman spectroscopy. J Ocul Pharmacol Ther, 2011. 27(5): p. 445-451.

16. Bauer, N.J., Motamedi, M., Wicksted, J.P., March, W.F., Webers, C.A., and Hendrikse, F., Non-invasive assessment of ocular pharmacokinetics using Confocal Raman Spectroscopy. J Ocul Pharmacol Ther, 1999. 15(2): p. 123-134.

17. Borchert, M.S., Storrie-Lombardi, M.C., and Lambert, J.L., A noninvasive glucose monitor: preliminary results in rabbits. Diabetes technology \& therapeutics, 1999. 1(2): p. 145-151.

18. Pelletier, C.C., Lambert, J.L., and Borchert, M., Determination of glucose in human aqueous humor using Raman spectroscopy and designed-solution calibration. Appl Spectrosc, 2005. 59(8): p. 1024-1031.

19. Sideroudi, T., Pharmakakis, N., Tyrovolas, A., Papatheodorou, G., Chryssikos, G.D., and Voyiatzis, G.A., Non-contact detection of ciprofloxacin in a model anterior chamber using Raman spectroscopy. J Biomed Opt, 2007. 12(3): p. 034005.

20. Hosseini, K., Jongsma, F.H., Hendrikse, F., and Motamedi, M., Non-invasive monitoring of commonly used intraocular drugs against endophthalmitis by Raman spectroscopy. Lasers in surgery and medicine, 2003. 32(4): p. 265-270. 
21. Jongsma, F.H.M., Erckens, R.J., Wicksted, J.P., Bauer, N.J.C., Hendrikse, F., March, W.F., and Motamedi, M. Confocal Raman spectroscopy system for noncontact scanning of ocular tissues: an in vitro study. 1997. SPIE.

22. US Pharmacopeia. USP Ketorolac tromethamine. 2018 [cited 2018 November 26]; Available from: http://www.pharmacopeia.cn/v29240/usp29nf24s0_m44006.html.

23. Hosseini, K., March, W., Jongsma, F.H., Hendrikse, F., and Motamedi, M., Noninvasive detection of ganciclovir in ocular tissue by Raman spectroscopy: implication for monitoring of drug release. J Ocul Pharmacol Ther, 2002. 18(3): p. 277-285.

24. Perez-Pueyo, R., Soneira, M.J., and Ruiz-Moreno, S., Morphology-based automated baseline removal for Raman spectra of artistic pigments. Appl Spectrosc, 2010. 64(6): p. 595-600.

25. Erckens, R.J., Motamedi, M., March, W.F., and Wicksted, J.P., Raman spectroscopy for noninvasive characterization of ocular tissue: Potential for detection of biological molecules. Journal of Raman Spectroscopy, 1997. 28(5): p. 293-299.

26. Zhang, X., Chen, S., Ling, Z., Zhou, X., Ding, D.Y., Kim, Y.S., and Xu, F., Method for Removing Spectral Contaminants to Improve Analysis of Raman Imaging Data. Sci Rep, 2017. 7: p. 39891.

27. Li, S. and Dai, L., An improved algorithm to remove cosmic spikes in Raman spectra for online monitoring. Appl Spectrosc, 2011. 65(11): p. 1300-1306.

28. Zhao, J., Lui, H., McLean, D.I., and Zeng, H., Automated autofluorescence background subtraction algorithm for biomedical Raman spectroscopy. Appl Spectrosc, 2007. 61(11): $p$. 1225-1232.

29. Allergan Inc., ACULAR® Prescribing Informaiton, in online. 2012. p. 6.

30. Lee, Y.H. and Lee, V.H.L., Formulation Influence on Ocular and Systemic Absorption of Topically Applied Atenolol in the Pigmented Rabbit. Journal of Ocular Pharmacology, 1993. 9(1): p. 47-58.

31. Bertens, C.J.F., Gijs, M., van den Biggelaar, F., and Nuijts, R., Topical drug delivery devices: A review. Exp Eye Res, 2018. 168: p. 149-160.

32. Del Amo, E.M., Rimpela, A.K., Heikkinen, E., Kari, O.K., Ramsay, E., Lajunen, T., Schmitt, M., Pelkonen, L., Bhattacharya, M., Richardson, D., Subrizi, A., Turunen, T., Reinisalo, M., Itkonen, J., Toropainen, E., Casteleijn, M., Kidron, H., Antopolsky, M., Vellonen, K.S., Ruponen, M., and Urtti, A., Pharmacokinetic aspects of retinal drug delivery. Prog Retin Eye Res, 2017. 57: p. 134-185.

33. Ling, T.L. and Combs, D.L., Ocular bioavailability and tissue distribution of [14C]ketorolac tromethamine in rabbits. J Pharm Sci, 1987. 76(4): p. 289-294.

34. Bucci, F.A., Jr. and Waterbury, L.D., Comparison of ketorolac $0.4 \%$ and bromfenac $0.09 \%$ at trough dosing: aqueous drug absorption and prostaglandin E2 levels. J Cataract Refract Surg, 2008. 34(9): p. 1509-1512.

35. Lázaro, J.C., Pacheco, M.T.T., Rodrigues, K.C., de Lima, C.J., Moreira, L.M., Villaverde, A.B., and Silveira Jr., L., Optimizing the Raman signal for characterizing organic samples: The effect of slit aperture and exposure time. Spectroscopy, 2009. 23(2).

36. Rangaraju, L.P., Kunapuli, G., Every, D., Ayala, O.D., Ganapathy, P., and MahadevanJansen, A., Classification of burn injury using Raman spectroscopy and optical coherence tomography: An ex-vivo study on porcine skin. Burns, 2018.

37. Jonas, O., Kang, J.W., Singh, S.P., Lammers, A., Nguyen, F.T., Dasari, R.R., So, P.T.C., Langer, R., and Cima, M.J., In vivo detection of drug-induced apoptosis in tumors using Raman spectroscopy. Analyst, 2018. 143(20): p. 4836-4839.

38. Kaji, Y., Akiyama, T., Segawa, H., Oshika, T., and Kano, H., Raman Microscopy: A Noninvasive Method to Visualize the Localizations of Biomolecules in the Cornea. Cornea, 2017. 36 Suppl 1: p. S67-S71.

39. Ammar, D.A., Lei, T.C., Kahook, M.Y., and Masihzadeh, O., Imaging the intact mouse cornea using coherent anti-stokes Raman scattering (CARS). Invest Ophthalmol Vis Sci, 2013. 54(8): p. 5258-5265.

40. Marro, M., Taubes, A., Abernathy, A., Balint, S., Moreno, B., Sanchez-Dalmau, B., MartinezLapiscina, E.H., Amat-Roldan, I., Petrov, D., and Villoslada, P., Dynamic molecular monitoring of retina inflammation by in vivo Raman spectroscopy coupled with multivariate analysis. J Biophotonics, 2014. 7(9): p. 724-734. 


\subsection{Supplementary figures}
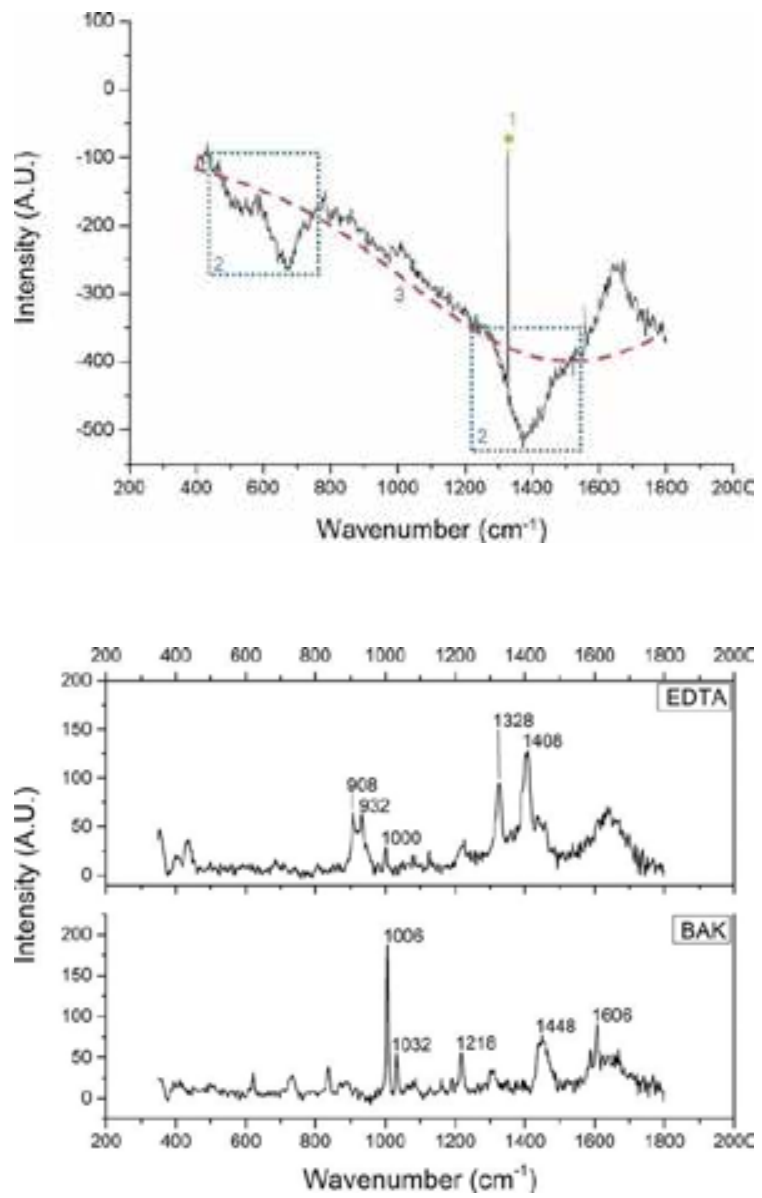

Figure 6-S1. Example of baseline correction of raw Raman spectra. (1) Cosmic ray correction, (2) correction for hardware-induced errors and (3) 5th degree polynomial correction for background fluorescence. $\mathrm{cm}-1=$ typically centimeters,
Figure 6-S2. Raman spectrum of the additives in Acular ${ }^{\mathrm{TM}}$. None of the peaks corresponds to the ketorolac spectrum. Only baseline correction is applied on the spectrum using polynomial correction. RAW data. Analyzed using OriginPro 9 64bit e.d. 

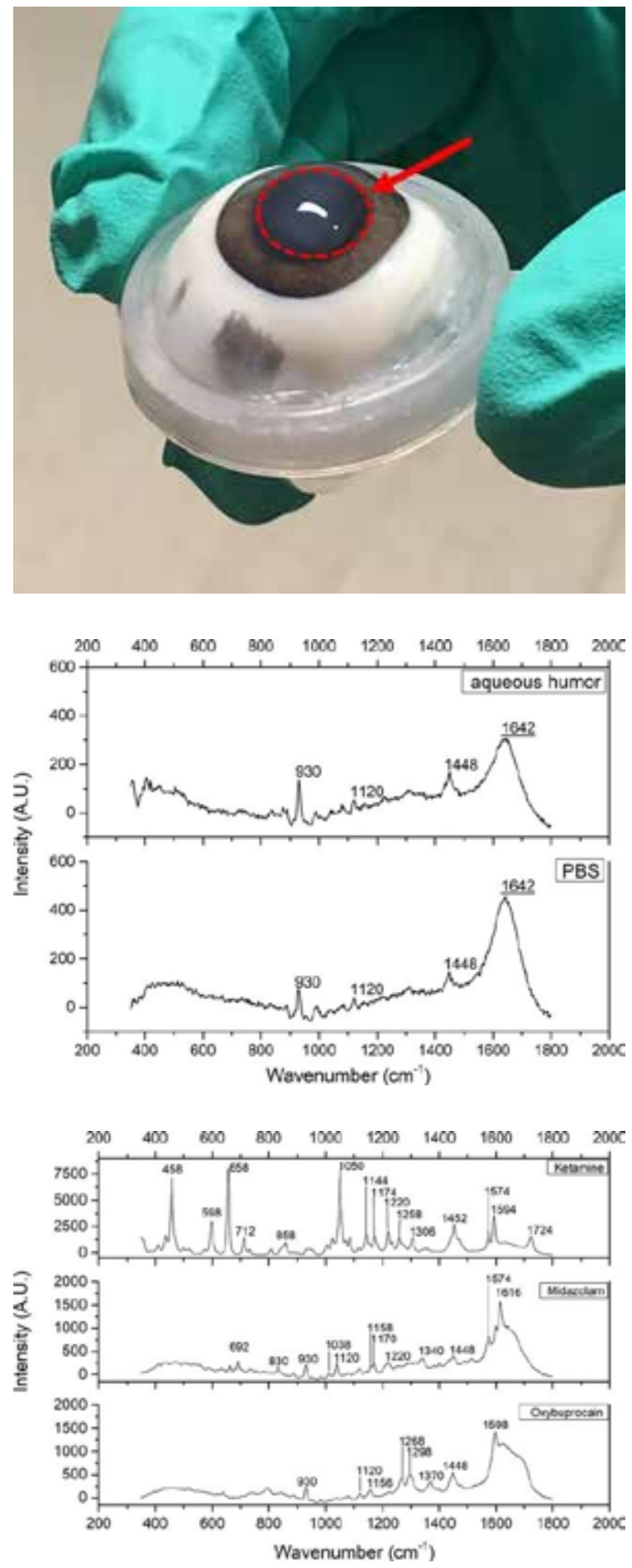

Figure 6-S3. Post mortem pig eye with a clearly visible corneal haze in the stromal layers.

Figure 6-S4. Raman spectrum of PBS and aqueous humor. The peaks in PBS and aqueous humor are similar to each other. Only baseline correction is applied on the spectrum using polynomial correction. RAW data. Analyzed using OriginPro 9 64bit e.d.

Figure 6-S5. Raman spectrum of ketamine, midazolam, and Oxybuprocaine hydrochloride. Only baseline correction is applied on the spectrum, using polynomial correction. RAW data. Analyzed using OriginPro 9 64bit e.d. 
Confocal Raman spectroscopy: evaluation of a non-invasive technique for the detection of topically applied ketorolac tromethamine in vitro and in vivo
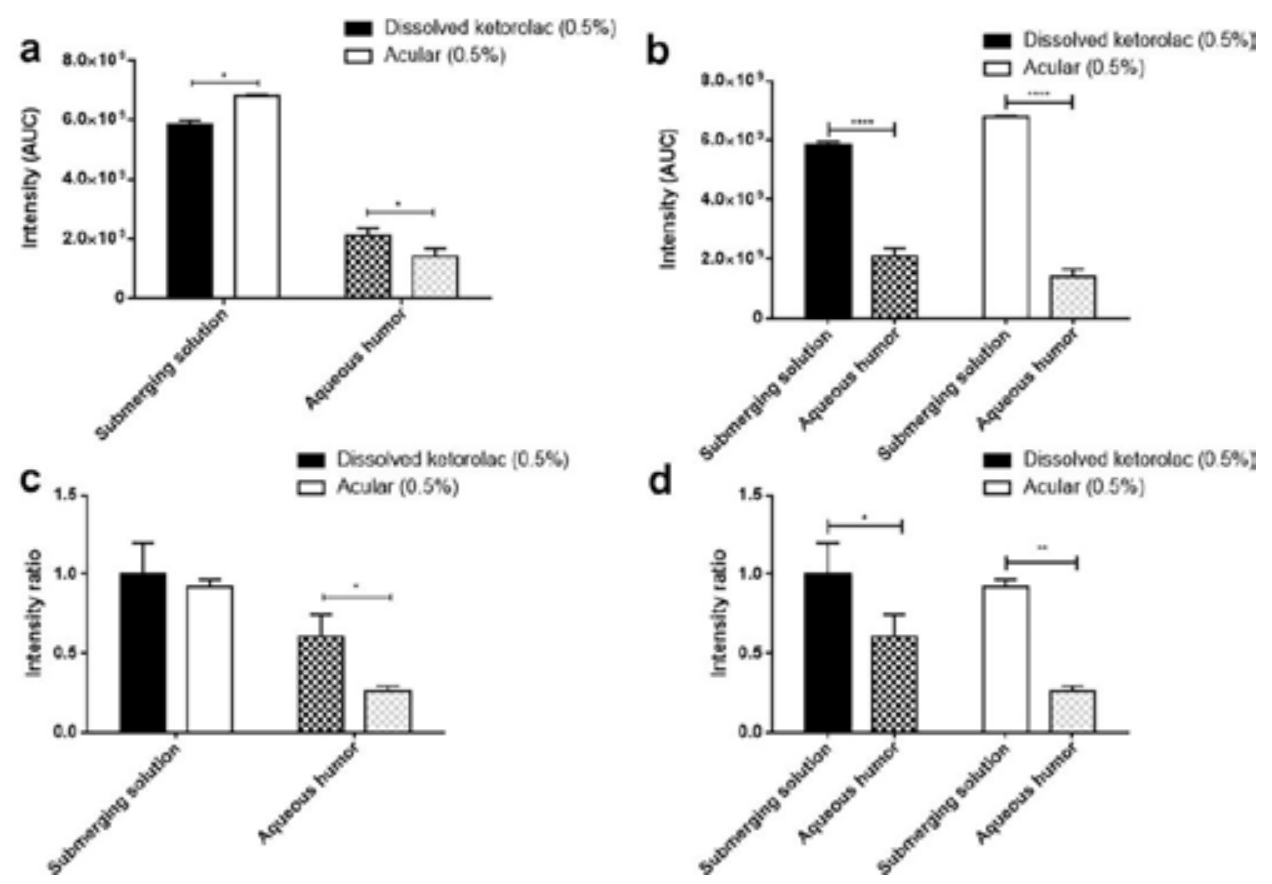

Figure 6-S6. Ocular penetration of dissolved ketorolac compared to Acular ${ }^{\mathrm{TM}}$ in pig eyes. (a) and (b) show HPLC analysis between Acular ${ }^{\mathrm{TM}}$ and dissolved ketorolac and graph (c) and (d) show the Raman data (cuvette detection). ${ }^{*} \mathrm{P}<0.05,{ }^{* *} \mathrm{P}<0.01$, ${ }^{* * *} \mathrm{P}<0.0001 \mathrm{n}=3$ per sample, data is plotted as mean $\pm \mathrm{SD}$. 


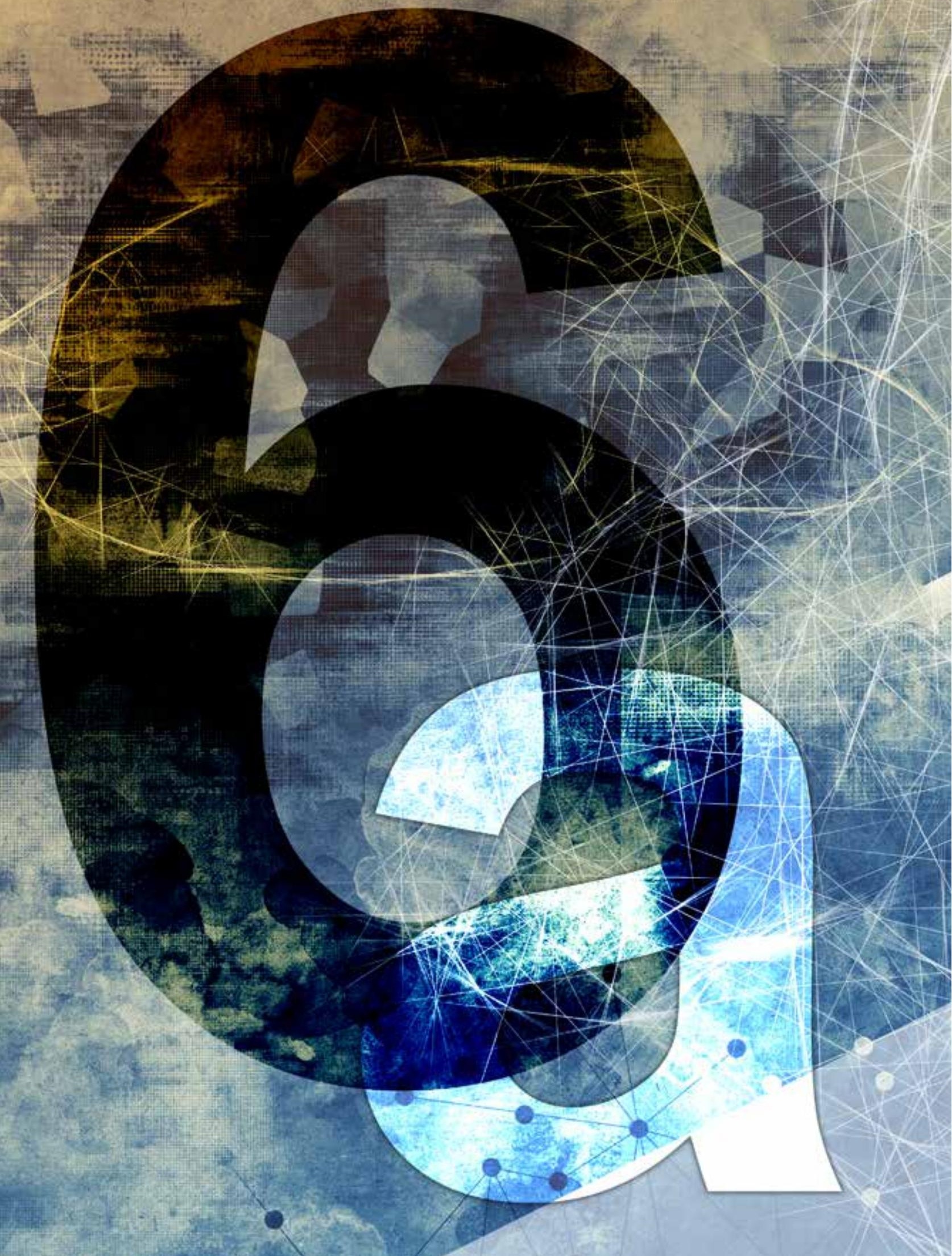


Chapter 6a.

\section{Pipeline for the removal of}

hardware related ambifacts and

background noise for Raman

\section{spectroscopy}

Christian J.F. Bertens, Shuo Zhang, Roel J. Erckens, Frank J.H.M. van den Biggelaar, Tos T.J.M. Bertendschot, Carroll A.B. Webers, Rudy M.M.A. Nuijts, and Marlies Gijs

MethodsX 2020 Apr 21;7:100883

DOI: $10.1016 /$ j.mex.2020.100883 


\section{Abstract}

Raman spectroscopy is a real-time, non-contact, and non-destructive technique able to obtain information about the composition of materials, chemicals, and mixtures. It uses the energy transfer properties of molecules to detect the composition of matter. Raman spectroscopy is mainly used in the chemical field because background fluorescence and instrumental noise affect biological (in vitro and in vivo) measurements. In this method, we describe how hardware related artifacts and fluorescence background can be corrected without affecting signal of the measurement. First, we applied manual correction for cosmic ray spikes, followed by automated correction to reduce fluorescence and hardware related artifacts based on a partial $5^{\text {th }}$ degree polynomial fitting and Tophat correction. Along with this manuscript we provide a MatLabß script for the automated correction of Raman spectra.

- "Polynomial_Tophat_background_subtraction_methods.m" offers an automated method for the removal of hardware related artifacts and fluorescence signals in Raman spectra.

- "Polynomial_Tophat_background_subtraction_methods.m" provides a modifiable MatLab file adjustable for multi-purpose spectroscopy analysis.

- We offer a standardized method for Raman spectra processing suitable for biological and chemical applications for modular confocal Raman spectroscope

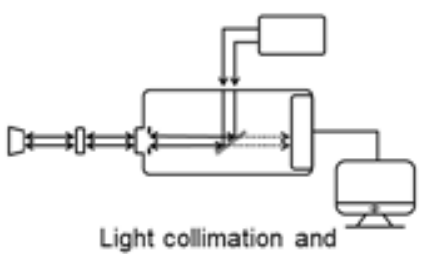

system calibration

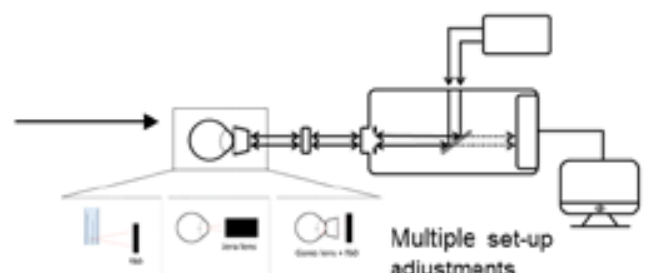

adjustments

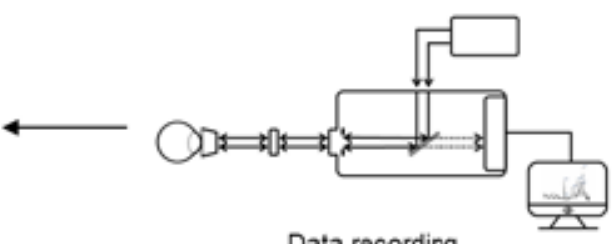

Data recording

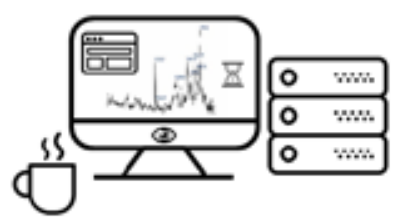

Data processing using:

Polynomial_tophot_bockground_subtraction _methods.m 


\section{6a.1. Introduction}

Raman spectroscopy is a vibrational spectroscopic technique, based on an energy transfer between an illuminated sample and the irradiated light. In contrast with e.g. infrared (IR) spectroscopy, which analyses absorbed and transmitted fractions of the light, Raman spectroscopy makes use of scattered radiation. Although the predominant mode of scattered light is elastic Rayleigh scattering, a small proportion ( 1 to $10^{9}$ or $10^{10}$ ) of the photons is scattered inelastically. These photons shift to a higher or lower energy status resulting in stokes and anti-stokes scattering.[1]

Raman spectra provides both qualitative and quantitative molecular-level information. The basis of the qualitative information is the fingerprint nature of the Raman shift, which is unique to each material. This makes Raman spectroscopy also usable in an aqueous environment [2], and an interesting and suitable technique for ophthalmic purposes. Raman spectroscopy is a non-contact and non-destructive technique with real-time visualisation, which make it also suitable for in vivo application.

Biological samples often emit fluorescence signals that may interfere with Raman signals since the intensity of the fluorescence emission has a much higher yield than Raman signals.[3] Further, hardware related artefacts (instrumental noise) are found in Raman spectra. In order to extract Raman signal from the raw acquired spectrum, it is therefore necessary to pre-process the acquired spectra.[4] As recognized by Byrne et al. no standardized protocols are available for this purpose yet.[5] Hence, we developed a method to deal with multiple source background influences. This paper guides you through the steps taken to optimize Raman spectra and make them ready for analysis as done in the study from Bertens et al.[6] For the full dataset of this project we refer to the supplementary data of Zhang et al.[7]

\section{6a.2. Background of the data processing}

As mentioned earlier, there is no gold standard for the processing of Raman data. Several approaches have been proposed to minimize the influence from background fluorescence.[5] Raman scattering is an instantaneous effect, whereas fluorescence requires time to occur. If one can switch on and off the detector (or a filter) at a high temporal resolution, fluorescence signal could be prevented from interfering with the Raman signal. However, this is expensive, complicated, and commercially not available.[8,9] Therefore, the most accepted method for fluorescence background subtraction is polynomial fitting, for which unfortunately no standardized protocols are available (figure 6a-1-3).[5] Zhao et al. introduced an automated polynomial background subtraction method for biomedical applications, which could subtract the background.[10] Zhang et al. also developed a proper automated method for fluorescence background subtraction named: "automatic pre-processing method for 
Raman imaging data set (APRI)".[11] However, both methods encountered difficulties when handling spectrums containing instrumental noise. In some in vivo experiments, the contribution from instrumental noise is inevitable and cannot be neglected, thus affecting the conventional polynomial methods. Hence, further treatments have been developed to eliminate the instrumental noise. Perez-Pueyo et al. introduced a morphology-based baseline removal method for Raman spectrums.[12] It employs Tophat filtering using basic operations as dilation and erosion to filter the features beyond or below a pre-set threshold, thereby removing the instrumental noise (figure 6a-1-2).

A third influencer affecting Raman spectra are cosmic rays. Cosmic rays create spikes that are randomly generated due to cosmic radiation (figure 6a-1-1). Cosmic rays affected different wavenumbers each time they occur, and can easily be detected by comparing different frames of one measurement. Spikes created by cosmic rays need to be removed before the frames are averaged, else they can be interpreted as peaks. $[4,11]$

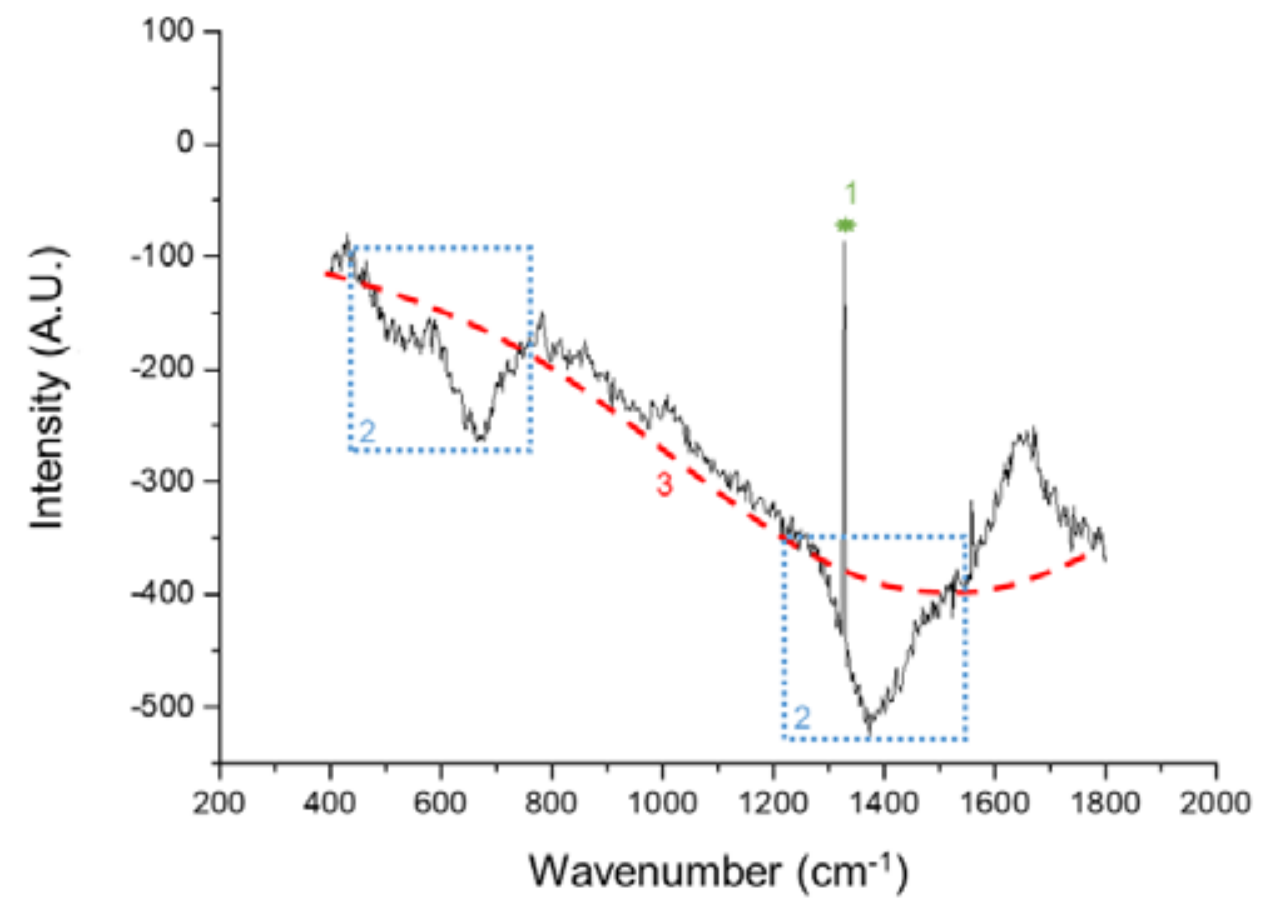

Figure 6a-1. Example of a Raman spectrum. (1, green) Shows a cosmic ray spike, (2, blue) shows instrumental influences on the spectrum, and $(3$, red $)$ shows a $5^{\text {th }}$ degree polynomial fitting for background correction. 


\section{6a.3. Materials and Methods}

\section{6a.3.1. Materials}

- Power conditioner: ONEAC PCm750I, 220-240V, 3.1A, 50/60Hz

- Laser with wavelength $785 \mathrm{~nm}$ : Laser Model SM $785 \mathrm{~nm}$ purchased from Innovative Photonic Solutions; Output Power $50 \mathrm{~mW}$; Bandwidth $9.73 \mathrm{GHz}$

- Laser with wavelength $671 \mathrm{~nm}$ : Laser model: Ignis 671, purchased from Laser quantum; Output Power 100 mW; Bandwidth $30 \mathrm{GHz}$

- Spectrometer: Model HPRM 2500, produced by River Diagnostics International BV. Specifications:

- Spectral region coverage: $350 \mathrm{~cm}^{-1}-1800 \mathrm{~cm}^{-1}$ with the $785 \mathrm{~nm}$ laser and $2500 \mathrm{~cm}^{-1}-4000 \mathrm{~cm}^{-1}$ with the $671 \mathrm{~nm}$ laser

- Spectral resolution: $2 \mathrm{~cm}^{-1}$ throughout the spectral region

- Pinhole size: 25 microns

- Back-illuminated deep-depletion CCD-camera: with 1024 x 128 pixels, air-cooled to $-60^{\circ} \mathrm{C}$. Camera control software included

- Computer: HP Compaq 6200 Pro Microtower with operation system Windows $\AA 7$ Pro OA

- Jena lens: Planachromat LD 25x/0.5 $/ 0$ (2)-A, focus length is $10.1 \mathrm{~mm}$

- Mirror: Beam steering mirror assembly, model G063713000

- Melles Griot Shear-plate

- Fibres: Diamond® FC APC/PM 20853190002 for $850 \mathrm{~nm}$ and FC APC/PM 20871100001 for $630 \mathrm{~nm}$

- GonioLens, Haag-Streit Meridian; CGA1

- Edmund Optics lenses: f60 (60 mm focus point), f80 (80 mm focus point)

\section{6a.3.2. Set-up of the Raman system}

A modular confocal Raman spectroscopic system was used in the study. The Raman system was connected via a power conditioner, to prevent power peaks to disturb the measurements and to protect the system. The Raman system is equipped with a diode-emitting laser of $785 \mathrm{~nm}$ with a continuous power of $26 \mathrm{~mW}$, and a $671 \mathrm{~nm}$ diode- emitting laser with a continuous power of $14 \mathrm{~mW}$. Raman spectra were recorded with a high-performance Raman module model 2500 with a chargecoupled device $(C C D)$ operating at $-60^{\circ} \mathrm{C}$. This module introduces the laser light through a diamond optical fiber, shapes and conditions the beam through a pinhole to the measurement stage (figure 6a-3). The emitting light from the spectrometer is collimated using a converging lens (f80 see figure 6a-3-f). Collimation of the light was checked using the Melles Griot shear-plate. The lens was moved along the laser optic axis towards or away from the exit aperture of the spectrometer until the stripes provide a collimated position (figure 6a-2). 


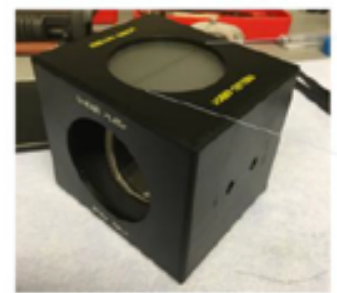

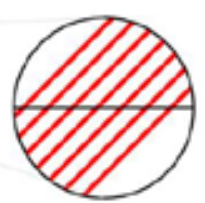

Diverging beam

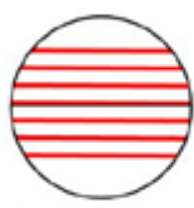

Collimated beam

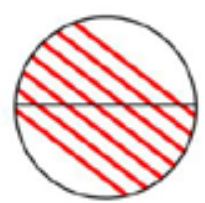

Converging beam

Figure 6a-2. Melles Griot shear plate and the patterns it provides with different types of emitted light.

Three types of sample set-ups were performed:

- Cuvette set-up (figure 6a-4a)

- In front of the sample, a f80 lens was used when the sample was measured in a Brand $\AA$ cuvette.

- Jena lens set-up (figure 6a-4b)

- In front of the sample, a long-working-distance microscope objective lens, Jena lens.

- Gonio lens set-up (figure 6a-4c)

- In front of the sample, first a lens with a f60 lens is placed, followed by a Gonio lens. The Gonio lens was connected to the cornea of an eye (in vivo or ex vivo) using topically applied Methocel囚 $2 \%$.

\section{6a.3.3. Calibration}

When the laser from the Raman system is collimated, the lens used for the measurement is set in place and the system is calibrated by built-in calibration procedure of the spectrometer. Hereafter, the system is further calibrated by the reference spectrum obtained by the provided National Institute of Standards and Technology (NIST)-standard calibration glass (was provided with the spectrometer). The full calibration was done according to the spectrometer manual. All measurements were performed in the dark.

\section{6a.3.4. Positioning}

The location in the sample was determined using the $671 \mathrm{~nm}$ laser, to create a high wave number signal (figure 6a-5a). In the eye, the cornea provides a protein peak $(2800 \mathrm{~cm}-1-3000 \mathrm{~cm}-1)$ followed by a water band $\left(3000 \mathrm{~cm}^{-1}-3800 \mathrm{~cm}^{-1}\right)$. The anterior chamber only has a water band $\left(3000 \mathrm{~cm}^{-1}-3800 \mathrm{~cm}^{-1}\right)$, and the lens has an extra protein peak around $3100 \mathrm{~cm}^{-1}$ besides the protein peak located at 2800 $\mathrm{cm}^{-1}-3000 \mathrm{~cm}^{-1}$ and a water peak at $3000 \mathrm{~cm}^{-1}-3800 \mathrm{~cm}^{-1}$. (figure $6 a-5 b$ ) In a cuvette, when focussed on the cuvette multiple high-intensity signals occur (between 
$\left.2000 \mathrm{~cm}^{-1}-3000 \mathrm{~cm}^{-1}\right)$. When focussed on the fluid in the cuvette a water peak occurs $\left(3000 \mathrm{~cm}^{-1}-3800 \mathrm{~cm}^{-1}\right)$.

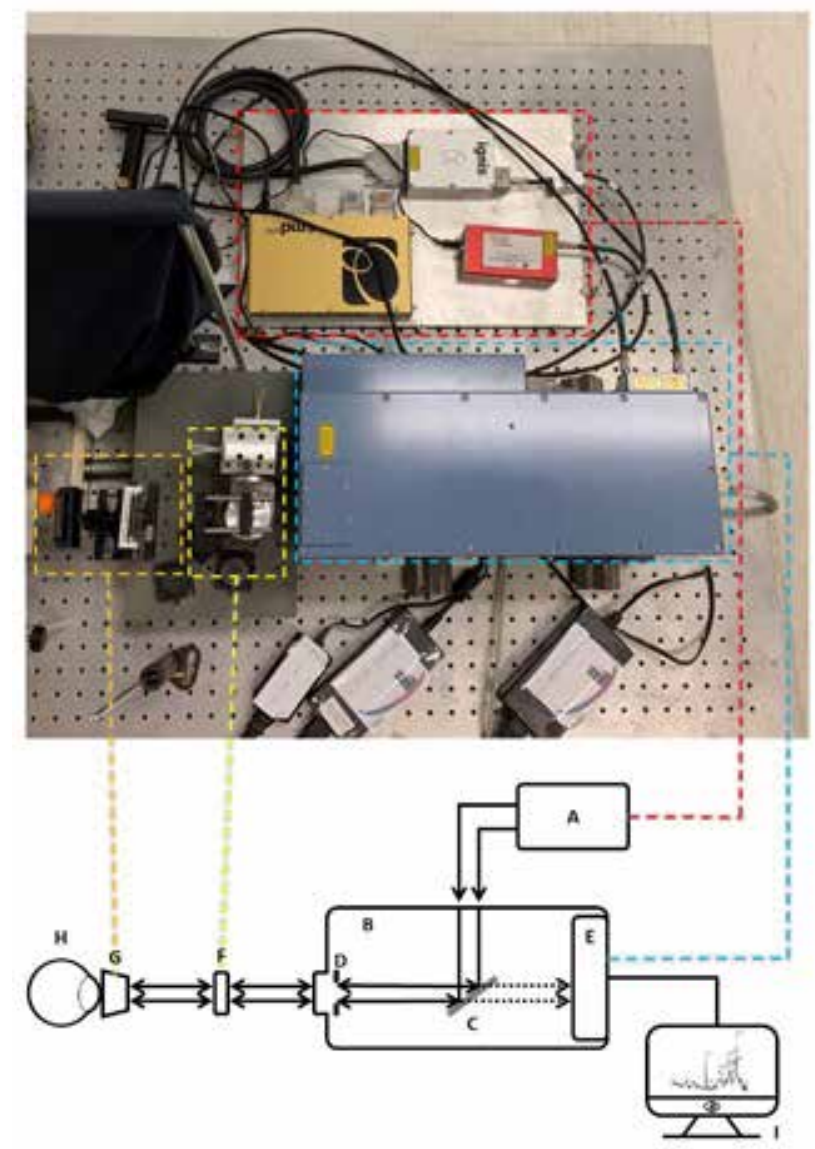

Figure 6a-3. Raman set-up. (A) laser (red dashed region); (B) Raman module (blue dashed region), with (C) filter for Raman scattered light, (D) $25 \mu \mathrm{m}$ pinhole and (E) integrated charge-coupled device (CCD); $(F)$ collimation f80 lens (yellow dashed region); (G) f60 lens with a Gonio (one-mirror) lens, or objective (Jena lens), or a f80 lens (orange dashed region); (H) sample; and (I) computer (didn't show in the photograph). Arrows indicate direction of (backscattered) laser light; dashed arrows indicate direction of Raman-Scattered light.

\section{6a.3.5. Data acquisition}

When the laser was correctly positioned, fingerprint-signal of the material was measured with the $785 \mathrm{~nm}$ laser and exported as '.txt' file further processing. An example of a measurement is provided in figure 6a-6.

\section{6a.4. Data processing}

\section{6a.4.1. Removal of cosmic ray spikes}

All Raman spectra were loaded into OriginPro 9.0.0 (64 bit ed. OriginLab corp. Northampton, US) and were one-by-one checked (manually) for cosmic ray spikes. The wavenumbers affected by cosmic ray spikes were replaced by the values of the same wavenumbers from another frame. When this was done, the files were saved 
and loaded into MatLab@ (Version 2017b, The Mathworks Inc., Natick, MA, US) for further processing.
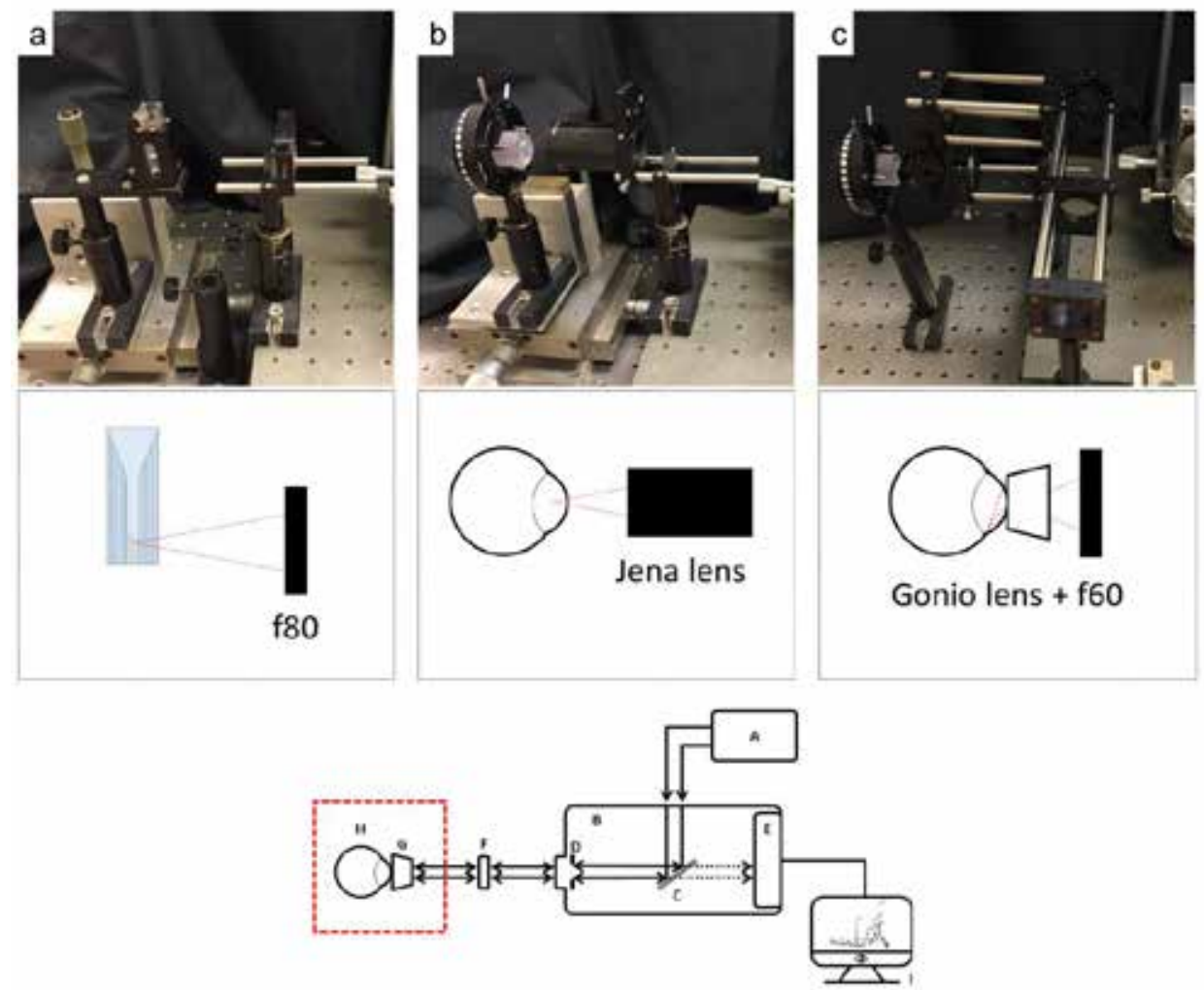

Figure 6a-4. Details of the focus area of the Raman system (red dashed line in the scheme). (a) The setup for cuvettes using a f80 lens, (b) Jena lens for focus in the anterior chamber of an eye, and (c) the Gonio lens in combination with a f60 focus lens for focus in the anterior chamber of an eye. The red dashed triangles show the focus position of the set-up.

\section{6a.4.2. Averaging of the frames, and removal of background noise and instrumental noise}

The following process is programmed in the MatLab@ file ("Polynomial_Tophat_ background_subtraction_methods.m"), provided with the manuscript.

First, frames were averaged to reduce fluctuations. Because the baseline has a strong influence on the polynomial approximation, the polynomial degree must be selected according to the shape of the baseline. In our system, using eyes, a $5^{\text {th }}$ degree polynomial fitting resulted in the most optimal background correction (figure 6a-S1). Therefore, we applied partial $5^{\text {th }}$ degree polynomial fitting with the morphology approach of Perez-Pueyo et al. [12] to remove instrumental noise. First, all spectra were dissected in different zones, $350 \mathrm{~cm}^{-1}$ to $450 \mathrm{~cm}^{-1}, 450 \mathrm{~cm}^{-1}$ to 750 
$\mathrm{cm}^{-1}, 750 \mathrm{~cm}^{-1}$ to $1250 \mathrm{~cm}^{-1}, 1250 \mathrm{~cm}^{-1}$ to $1650 \mathrm{~cm}^{-1}$, and $1650 \mathrm{~cm}^{-1}$ to $1800 \mathrm{~cm}^{-1}$. Zones that only contain fluorescence $\left(400 \mathrm{~cm}^{-1}\right.$ to $450 \mathrm{~cm}^{-1}, 800 \mathrm{~cm}^{-1}$ to $1200 \mathrm{~cm}^{-1}$, and $1600 \mathrm{~cm}^{-1}$ to $1800 \mathrm{~cm}^{-1}$ )(figure $6 a-7$, zone 1,2 , and 3) are used calculate the polynomial function coefficients. The zone containing the water-peak $\left(1550 \mathrm{~cm}^{-1}\right.$ to $1650 \mathrm{~cm}^{-1}$ ) was excluded from the polynomial function fitting calculation. The achieved $5^{\text {th }}$ degree polynomial function was applied on the full spectrum $\left(400 \mathrm{~cm}^{-1}\right.$ to $1700 \mathrm{~cm}^{-1}$ ) to remove the fluorescence background (figure 6a-7). Hereafter, the morphology-based Tophat method from Perez-Pueyo et al. [12] was applied to eliminate instrumental noise. Examples of processed Raman signals are shown in figure 6a-8.

Figure 6a-9 shows the effect of data processing using the MatLab $\odot$ program on a sample without (figure 6a-9a) and with (figure 6a-9b) instrumental noise. In both occasions, a flat baseline is observed, and in figure $9 b$ instrumental noise is reduced without affecting the peaks. A full overview of the corrected data can be found in Bertens et al. [6], and the full data-set is available supplementary to the manuscript from Zhang et al.[7]
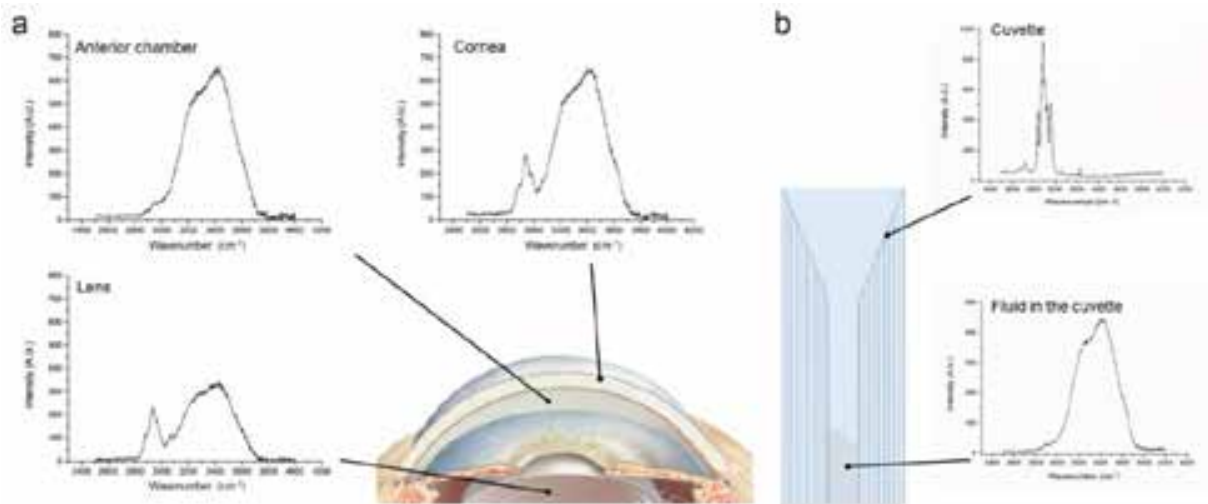

Figure 6a-5. Location determination using high wave number measurement (671 nm Laser). (a) In the eye, and in (b) a cuvette 

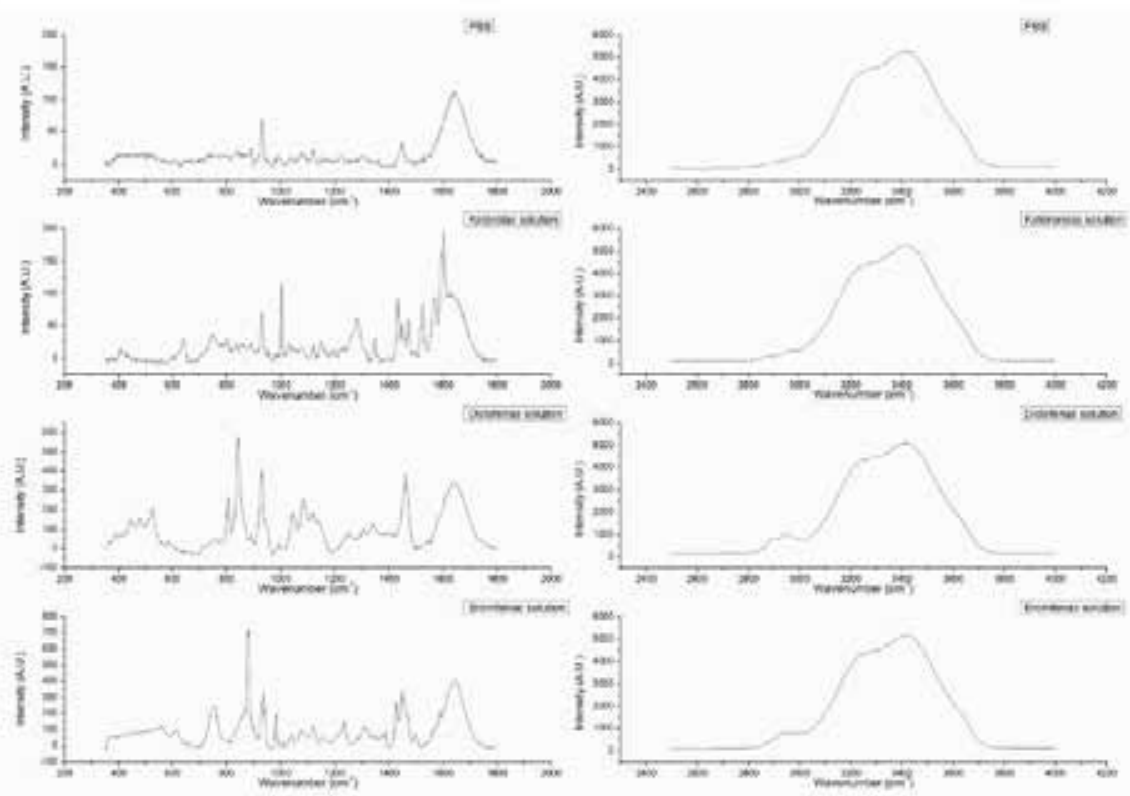<smiles>O=C(c1ccccc1)c1ccc2n1CCC2C(=O)O</smiles>

Ketorolac<smiles>Nc1c(CC(=O)O)cccc1C(=O)c1ccc(Br)cc1</smiles>

Bromfenac<smiles>O=C(O)Cc1ccccc1Nc1c(Cl)cccc1Cl</smiles>

Diclofenac

Figure 6a-6. Raman spectra providing fingerprint signal (left column) and a high wave number signal (right column) of PBS and three different drugs (ketorolac (Acular $\AA$ ), Bromfenac (Yellox®), and Diclofenac $($ Naclof $\AA)$ ) in ophthalmic solution. With corresponding molecular structure.
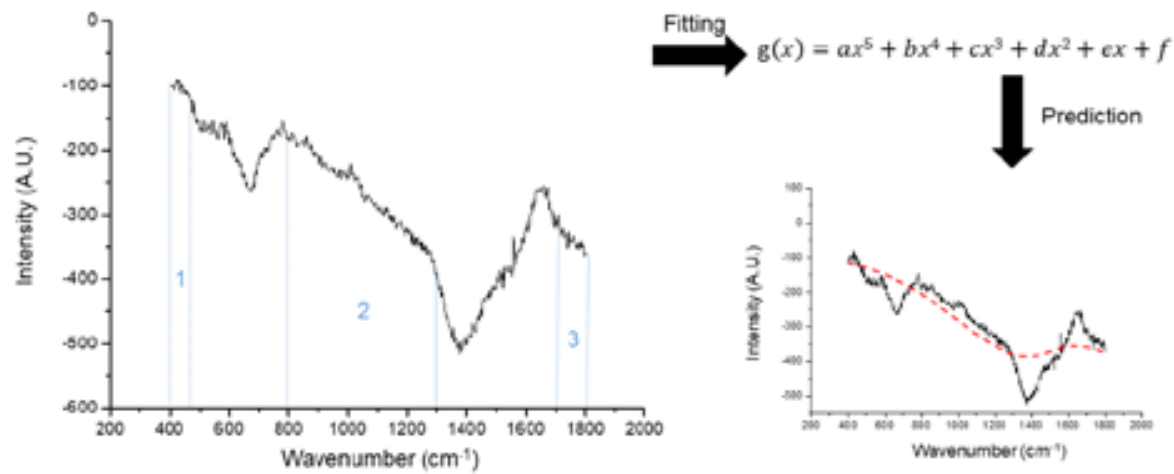

Figure 6a-7. Overview of partial polynomial fitting. The spectrum is divided into different zones $(1,2$, and 3 ), where after, a line was fitted through those zones based on a $5^{\text {th }}$ order polynomial function. The predicted line was withdrawn from the graph. 
a
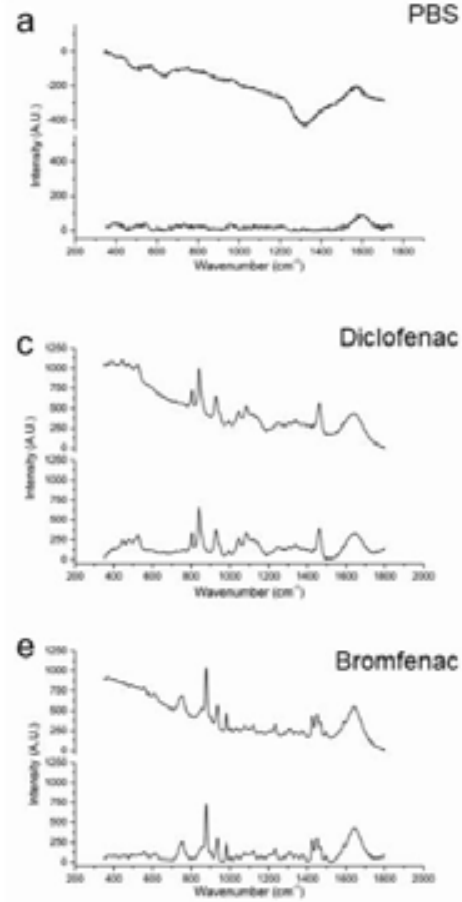

PBS
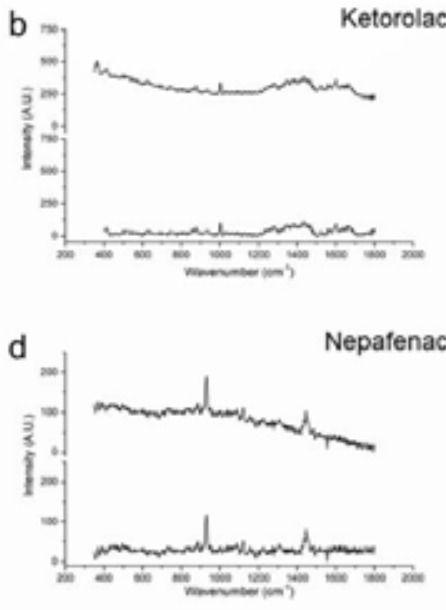

Figure 6a-8. Effect of the processing on the data. (a) PBS in a rabbit eye, (b) ketorolac in a porcine eye, (c) diclofenac in a porcine eye, (d) nepafenac in a porcine eye, and (e) bromfenac in a porcine eye. The upper line shows RAW Raman signal and the lower line represents a processed Raman signal. Exposure time 30 s, average of 3 frames.

a

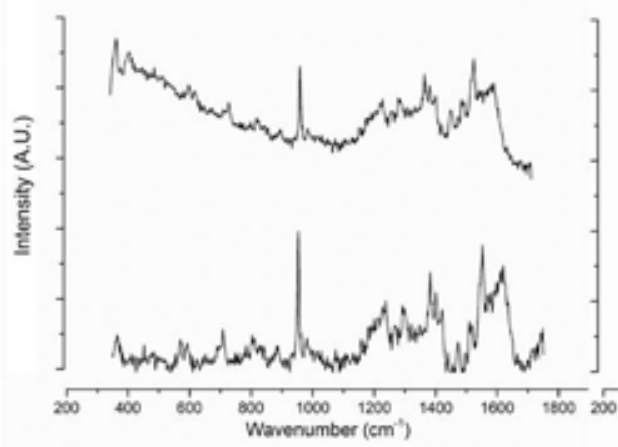

b
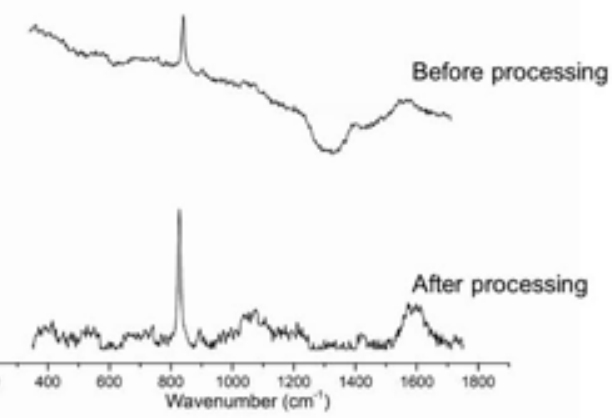

Figure 6a-9. Effect of the processing on instrumental influences. (a) Shows a graph without instrumental influences (PBS) and (b) shows a graph with instrumental influences (rabbit eye). The upper line shows a RAW Raman spectrum and the lower line represents a processed Raman spectrum. Sample (a) is an ex vivo porcine eye, measured with Gonio lens, treated with $1.25 \%$ ketorolac tromethamine (ophthalmic solution), exposure time 60s, 3 frames. Sample (b) is an in vivo measurement of a rabbit eye (non-treated), measured with Gonio lens, exposure time 30s, 2 frames. 


\section{6a.5. References}

1. Long, D.A., Handbook of Raman spectroscopy. From the research laboratory to the process line, in Journal of Raman Spectroscopy, I.R.L.a.H.G.M. Edwards., Editor. 2004, Marcel Dekker: New York and Basel. p. 91-91.

2. Esmonde-White, K.A., Cuellar, M., Uerpmann, C., Lenain, B., and Lewis, I.R., Raman spectroscopy as a process analytical technology for pharmaceutical manufacturing and bioprocessing. Analytical and Bioanalytical Chemistry, 2017. 409(3): p. 637-649.

3. Buckley, K. and Ryder, A.G., Applications of Raman Spectroscopy in Biopharmaceutical Manufacturing: A Short Review. Appl Spectrosc, 2017. 71(6): p. 1085-1116.

4. Hosseini, K., March, W., Jongsma, F.H., Hendrikse, F., and Motamedi, M., Noninvasive detection of ganciclovir in ocular tissue by Raman spectroscopy: implication for monitoring of drug release. J Ocul Pharmacol Ther, 2002. 18(3): p. 277-285.

5. Byrne, H.J., Knief, P., Keating, M.E., and Bonnier, F., Spectral pre and post processing for infrared and Raman spectroscopy of biological tissues and cells. Chem Soc Rev, 2016. 45(7): p. 1865-1878.

6. Bertens, C.J.F., Zhang, S., Erckens, R.J., van den Biggelaar, F., Berendschot, T., Webers, C.A.B., Nuijts, R., and Gijs, M., Confocal Raman spectroscopy: Evaluation of a non-invasive technique for the detection of topically applied ketorolac tromethamine in vitro and in vivo. Int $\mathrm{J}$ Pharm, 2019. 570: p. 118641.

7. Zhang, S., Bertens, C.J.F., Erckens, R.J., van den Biggelaar, F., Berendschot, T., Webers, C.A.B., Nuijts, R., and Gijs, M., In vitro and in vivo datasets of topically applied ketorolac tromethamine in aqueous humor using Raman spectroscopy. Data Brief, 2019. 27: p. 104694.

8. Littleford, R.E., Matousek, P., Towrie, M., Parker, A.W., Dent, G., Lacey, R.J., and Smith, W.E., Raman spectroscopy of street samples of cocaine obtained using Kerr gated fluorescence rejection. Analyst, 2004. 129(6): p. 505-506.

9. Martyshkin, D.V., Ahuja, R.C., Kudriavtsev, A., and Mirov, S.B., Effective suppression of fluorescence light in Raman measurements using ultrafast time gated charge coupled device camera. Review of Scientific Instruments, 2004. 75(3): p. 630-635.

10. Zhao, J., Lui, H., McLean, D.I., and Zeng, H., Automated autofluorescence background subtraction algorithm for biomedical Raman spectroscopy. Appl Spectrosc, 2007. 61(11): p. 1225-1232.

11. Zhang, X., Chen, S., Ling, Z., Zhou, X., Ding, D.Y., Kim, Y.S., and Xu, F., Method for Removing Spectral Contaminants to Improve Analysis of Raman Imaging Data. Sci Rep, 2017. 7: p. 39891.

12. Perez-Pueyo, R., Soneira, M.J., and Ruiz-Moreno, S., Morphology-based automated baseline removal for Raman spectra of artistic pigments. Appl Spectrosc, 2010. 64(6): p. 595-600. 


\section{6a.6. Supplementary material}

Matlab ${ }^{\odot}$ script: "Polynomial_Tophat_background_subtraction_methods.m". This script can be downloaded from the journal's website:

https://doi.org/10.1016/j.mex.2020.100883

a

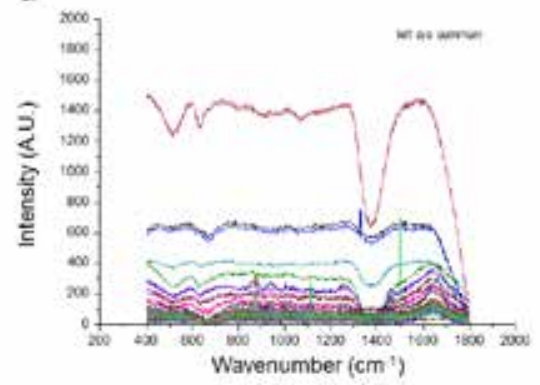

b
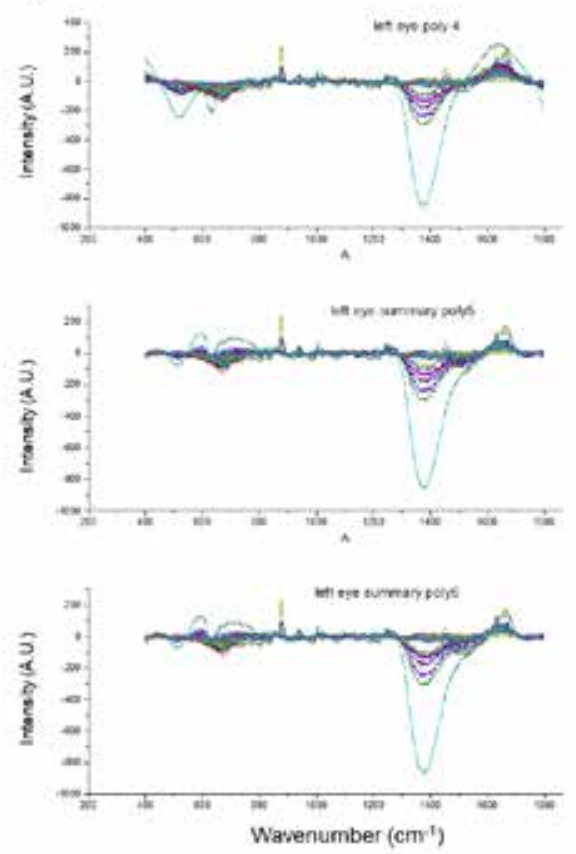

Figure 6a-S1. Optimization of the polynomial fitting. (a) RAW data before polynomial correction. (b) Data corrected using a $4^{\text {th }}$, a $5^{\text {th }}$, and a $6^{\text {th }}$ polynomial fitting function as shown in figure 5 . No difference between the $5^{\text {th }}$ and $6^{\text {th }}$ polynomial fitting was observed; therefore, $5^{\text {th }}$ order polynomial fitting was used during the corrections. 


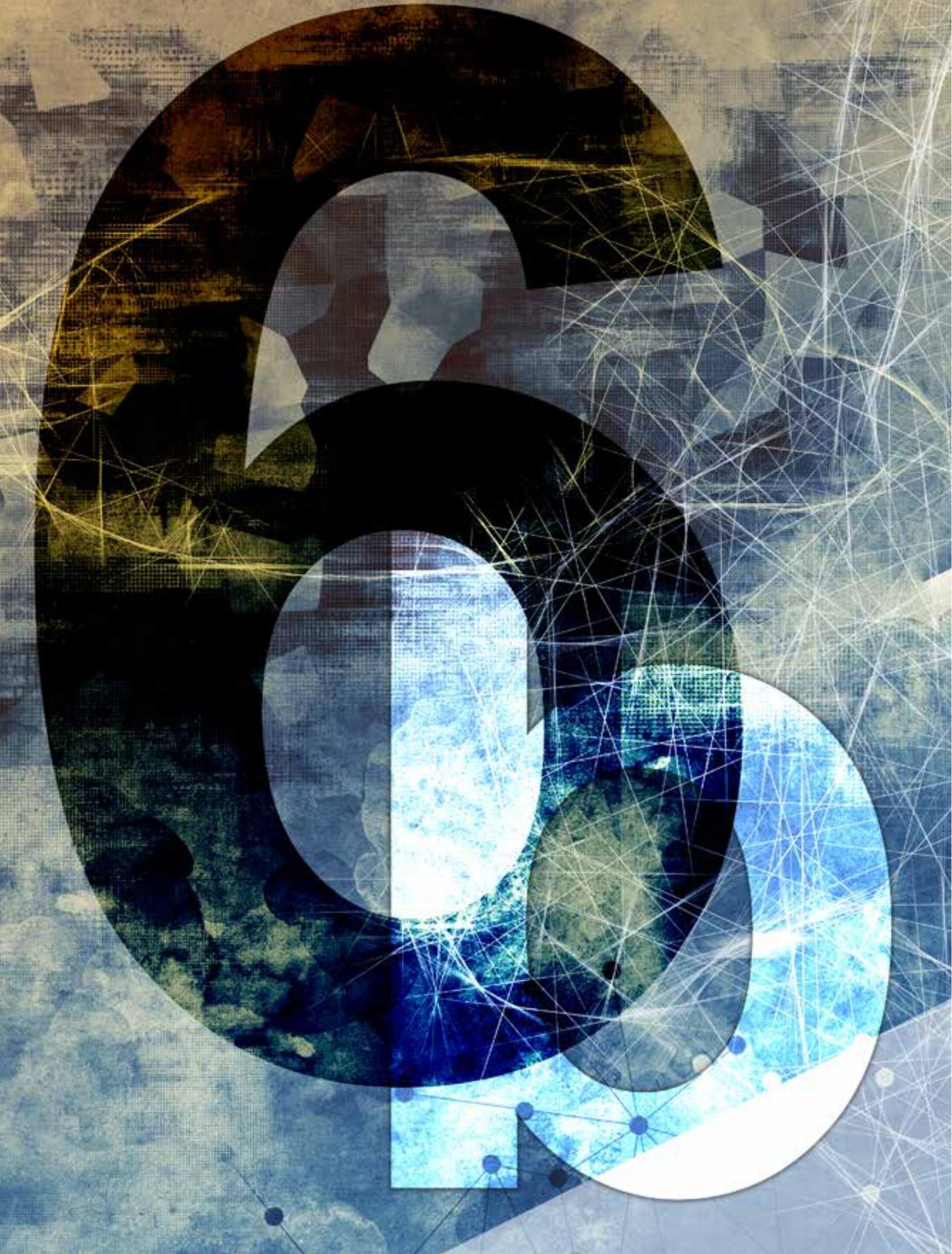


Chapter 6b.

In vintro and in vivo datasets of \{opically applied ketorolac tromethamine in aqueous humor using Raman spectroscopy

Shuo Zhang, Christian J.F. Bertens, Roel J. Erckens, Frank J.H.M. van den Biggelaar, Tos T.J.M. Bertendschot, Carroll A.B. Webers, Rudy M.M.A. Nuijts, and Marlies Gijs

Data in Brief. 2019 Oct 22; 104694.

DOI: $10.1016 / j . d i b .2019 .104694$ 


\section{Abstract}

This article includes datasets acquired by Raman spectroscopy from in vivo and in vitro ocular samples collected from the dataset from Bertens et al., "Confocal Raman spectroscopy: Evaluation of a non-invasive technique for the detection of topically applied ketorolac tromethamine in vitro and in vivo".[1] Detection of ketorolac tromethamine in pig eyes was performed in vitro and rabbit eyes in vivo. Extracted aqueous humor samples from pig and rabbit eyes were measured in vitro using a cuvette. This manuscript shows the spectral Raman data without pre-treatment or analysis from ocular tissues and provides further information towards aqueous humor research via alternative data processing methods. Furthermore, the raw data enclosed may be used for future aqueous humor investigations and pharmaceutical research.

\section{Value of the Data}

- The dataset could be used for further composition analysis of the aqueous humor and for future pharmaceutical research, to increase sensitivity of Raman systems.

- The dataset can be useful for researchers who are interested in the aqueous humor composition, ocular pharmaceutics, Raman spectroscopy, and software engineers.

- Alternative processing methods could be applied to exact other compounds in the aqueous humor or to enhance signals.

- This dataset offers a large cohort of animals measured on both eyes, 5 times. 


\section{6b.1 Data}

The data contains unanalysed Raman spectra obtained from pig eyes (in vitro) (6b.1.1, see supplementary files folder "in vitro pig eyes" and "in vitro cuvettes, aqueous humor from pig eyes"), rabbit eyes (in vivo) (6b.1.2, see supplementary files folder "in vivo rabbit eyes"), and aqueous humor samples (in vitro, see supplementary files folder "in vitro cuvettes, aqueous humor from rabbit eyes", $6 b .1 .3)$. Based on the differences of the samples, three types of set-ups were used on each dataset. For pig eye measurements in vitro, a long-working-distance microscope objective lens (Jena lens alone or a Gonio lens combined with a f60 lens) was utilized (see supplementary files "in vitro pig eyes" folder "jena lens" or "gonio"). For the rabbit eyes measurements in vivo, a Gonio lens combine with a f60 lens was used. For cuvettes measurements, a f80 lens was used when the sample was measured in a Brand ${ }^{\circledR}$ cuvette.[2] For each experimental set-up, the fingerprintwavenumber region (patterns specific for a drug-molecule, ranging from $350 \mathrm{~cm}^{-1}$ to $1800 \mathrm{~cm}^{-1}$ ) and the high-wavenumber region (higher energy shifted, ranging from $2500 \mathrm{~cm}^{-1}$ to $4000 \mathrm{~cm}^{-1}$ ) were included. The fingerprint spectra dataset was used for detection of intraocular ketorolac tromethamine as described in the article of Bertens et al.[1] Several peaks could be identified in the fingerprint region spectrum of a ketorolac tromethamine sample (figure 6b-1a). Only major peaks specific for ketorolac tromethamine were selected. Those peaks are assigned to certain chemical bonds or vibration modes. The assignment of the ketorolac related peaks is presented in Table 6b-1.[1] Due to the spectrometer's spectral resolution $\left(2 \mathrm{~cm}^{-1}\right)$, the peak observed at $1586 \mathrm{~cm}^{-1}$ is assigned to $\mathrm{NH}_{2}$ deformation.[3] The peak of 1524 $\mathrm{cm}^{-1}$ is assigned to in-plane vibrations of the conjugated $-\mathrm{C}=\mathrm{C}$-. The observed peak at $1472 \mathrm{~cm}^{-1}$ is assigned to $\mathrm{C}=\mathrm{N}$ stretching and the peak at $1282 \mathrm{~cm}^{-1}$ is assigned to $\mathrm{CH}_{2}$ wagging vibrations. Because Raman spectrum of the cornea, aqueous humor, and lens show different patterns in the high-wavenumber region, spectra from this region could be used as guide for location determination in the ocular tissue (figure $6 b-1 b) \cdot[4-6]$

\section{6b.1.1 In vitro, dataset}

Pig eyes (enucleated) were immersed in the dark at $4^{\circ} \mathrm{C}$ for 24 hours in vitro in different concentrations of ketorolac solutions $(0.05 \%$ to $5.0 \%)$ before the measurements (see supplementary files folder 'in vitro pig eyes'). For each concentration, three eyes were measured by Raman spectroscopy. An example spectrum obtained from a pig eye is shown in figure $6 b-2$. The location in the eye was determined using the high-wavenumber spectra (figure $6 b-2 b$ ). 


\section{6b.1.2 In vivo dataset}

New Zealand white rabbits received $50 \mu \mathrm{L}$ Acular $^{\circledR}$ three times a day in their right eye. At the same time, they received a drop of buffered saline solution (BSS) in their left eye as a control (see supplementary files folder "in vivo rabbit eyes"). The measurement parameters of the Raman system were optimized using the first four rabbits. Different integration times $(10,15$, or 30 seconds) were measured to acquire the optimum Raman signal. The following measurements were performed using an integration time of 30 seconds. During these measurements, hardware influences were observed. Further optimization of the processing method can be seen in Bertens et al.[2] The difference of the variant integration times can be found in figure $6 b-3$, for example, the spectrum intensity at $400 \mathrm{~cm}^{-1}$ is from 74 A.U. with 10 second integration time (figure 6b-3a), 127 A.U. with 15 second integration time (figure 6b$3 b$ ) and 333 A.U. with 30 second integration time (figure 6b-3c). Rabbits were measured according to the schedule in Table $6 b-2$.
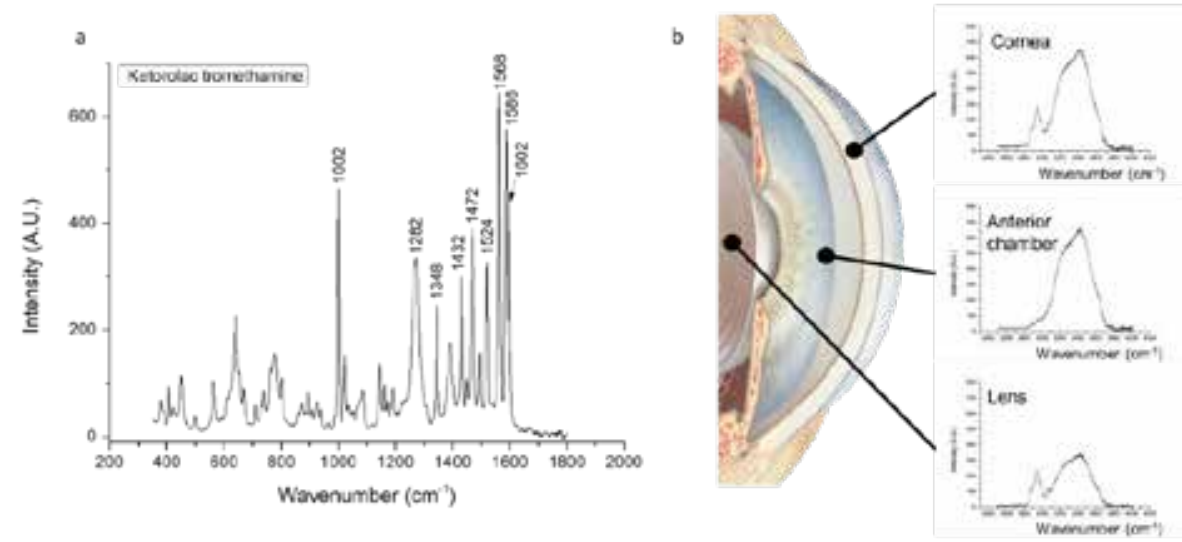

Figure 6b-1. (a) Fingerprint spectra of Ketorolac powder. (b) Determination of the location in the eye using high-wavenumber Raman spectra. Spectra are from pig eyes, 3 frames of 10 seconds averaged measured using a Jena lens. 
Table 6b-1. Main characteristic bands assignment of ketorolac [7]

\begin{tabular}{|c|c|c|}
\hline $\begin{array}{l}\text { Peak location } \\
\text { (wavenumber) }\end{array}$ & Intensity & Peak Assignment \\
\hline $1002 \mathrm{~cm}^{-1}$ & $\begin{array}{l}\text { very } \\
\text { strong }\end{array}$ & $\begin{array}{l}\text { Phenylalanine or a C-C aromatic ring } \\
\text { stretching }\end{array}$ \\
\hline $1282 \mathrm{~cm}^{-1}$ & medium & $\mathrm{CH}_{2}$ wagging vibrations \\
\hline $1348 \mathrm{~cm}^{-1}$ & weak & An unassigned mode \\
\hline $1432 \mathrm{~cm}^{-1}$ & strong & $\mathrm{CH}$ bond $[5]$ \\
\hline $1472 \mathrm{~cm}^{-1}$ & medium & $\mathrm{C}=\mathrm{N}$ stretching \\
\hline $1524 \mathrm{~cm}^{-1}$ & medium & In-plane vibrations of the conjugated $-\mathrm{C}=\mathrm{C}-$ \\
\hline $1568 \mathrm{~cm}^{-1}$ & $\begin{array}{l}\text { very } \\
\text { strong }\end{array}$ & $\mathrm{COO}^{-}$ \\
\hline $1586 \mathrm{~cm}^{-1}$ & strong & $\mathrm{NH}_{2}$ deformation [3] \\
\hline $1602 \mathrm{~cm}^{-1}$ & medium & Phenylalanine or a $\mathrm{C}==\mathrm{C}$ bond. \\
\hline
\end{tabular}
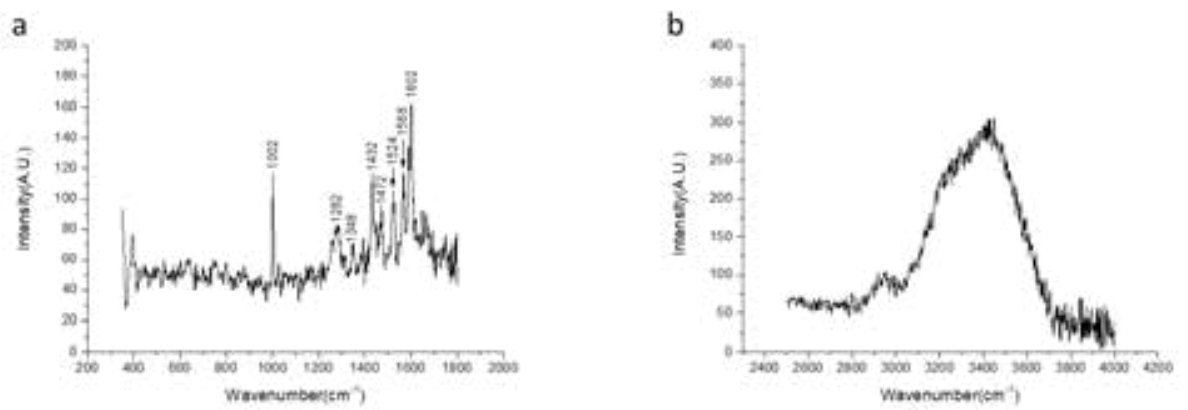

Figure $6 \mathrm{~b}-2$. Raman spectrum of a pig eye soaked in a $5 \%$ ketorolac solution obtained by Jena lens. (a) Fingerprint spectrum, obtained using 60 seconds and averaged for 3 frames. (b) High-wavenumber spectrum, obtained using 60 seconds and averaged for 3 frames. No correction has been applied on the spectra.
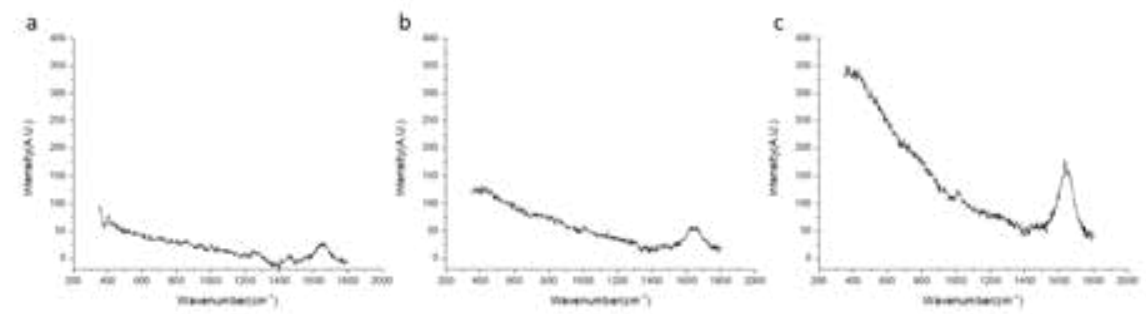

Figure 6b-3. In vivo Raman spectrum of the right eye of a rabbit with different integration times, averaged for 2 frames. (a) Shows the graph for 10 seconds, (b) 15 seconds, and (c) 30 seconds. 
Table 6b-2. In vivo integration time of the Raman measurements of the rabbits

$\begin{array}{lllllll}\text { No. } & \text { Name } & \text { Day 0 } & \text { Day } 7 & \text { Day } 14 & \text { Day 21 } & \text { Day 28 } \\ 1 & \text { PLAC } & x & 10 s & 15 s & 15 s & 30 s \\ 2 & \text { PLBT } & 10 s & x & 15 s & 15 s & 30 s \\ 3 & \text { PKXF } & 10 s & 10 s & 15 s & 15 s & 30 s \\ 4 & \text { PKYJ } & 10 s & 10 s & 15 s & 15 s & 30 s \\ 5 & \text { PNRS } & 30 s & 30 s & 30 s & 30 s & 30 s \\ 6 & \text { PNPH } & 30 s & x & & & \\ 7 & \text { PNPJ } & 30 s & 30 s & 30 s & 30 s & 30 s \\ 8 & \text { PNLJ } & 30 s & 30 s & 30 s & 30 s & 30 s \\ 9 & \text { POLI } & 30 s & x & & & \\ 10 & \text { POBS } & 30 s & 30 s & 30 s & 30 s & 30 s \\ 11 & \text { PPDI } & 30 s & 30 s & 30 s & 30 s & 30 s \\ 12 & \text { POHI } & 30 s & 30 s & 30 s & 30 s & 30 s\end{array}$

Integration time is shown in seconds, ' $x$ ' represents a failed measurement or no data. 2 frames per measurement were used.

\section{6b.1.3 In vitro, cuvettes dataset}

Immediately after intra-ocular Raman measurements (both in vitro and in vivo), 100 $\mu \mathrm{L}$ to $150 \mu \mathrm{L}$ of aqueous humor was drawn from the pig eyes, and $50 \mu \mathrm{L}$ was drawn from the right eye of each rabbit. The aqueous humor samples were frozen on dry ice and stored in a $-80^{\circ} \mathrm{C}$ freezer until use. When used, the location of focus was determined with the high wavenumber spectra, as shown in figure $6 b-4$.

Fingerprint spectra were collected to determine ketorolac concentrations in the aqueous humor. Spectrum examples of pig and rabbit aqueous humor are show in figure $6 b-5 a$ and figure $6 b-5 b$, respectively (see supplementary files folder "in vitro cuvettes"). Further background subtraction needs to be applied for analyses.

\section{6b.2 Experimental design, materials, and methods}

\section{6b.2.1 Raman spectroscopy system}

Two diode lasers were utilized as an excitation light source for Raman spectroscopy: a $26 \mathrm{~mW} 785 \mathrm{~nm}$ laser (Innovative Photonic Solutions SM $785 \mathrm{~nm}$, Monmouth Junction, NJ, US) or a $14 \mathrm{~mW} 671 \mathrm{~nm}$ laser (Laser Quantum Ignis 671 and SMD 6000, Konstanz, DE). A high-performance Raman spectrometer module (model 2500, River Diagnostics ${ }^{\circledR}$, Rotterdam, NL) was utilized for Raman spectra recordings.[8] A $25 \mu \mathrm{m}$ diameter pinhole was integrated within the spectrometer for the confocal Raman spectroscopy detection. An air-cooled charge-coupled device (CCD) camera with operating temperature $-60^{\circ} \mathrm{C}$ was integrated within the 
spectrometer for signal detection. The Raman spectrometer is capable of collecting Raman scattering wavenumber ranges in $350 \mathrm{~cm}^{-1}-1800 \mathrm{~cm}^{-1}$ and $2500 \mathrm{~cm}^{-1}-4000$ $\mathrm{cm}^{-1}$ with $2 \mathrm{~cm}^{-1}$ spectral resolution. A diverged laser beam out of the spectrometer is converted to a collimation beam by a lens with focus length of $80 \mathrm{~mm}$ (f80). Depending on the measurement, the lens setup was adapted. The system was used in single point modus and location in the sample was determined using the high wave numbers (671 $\mathrm{nm}$ laser).

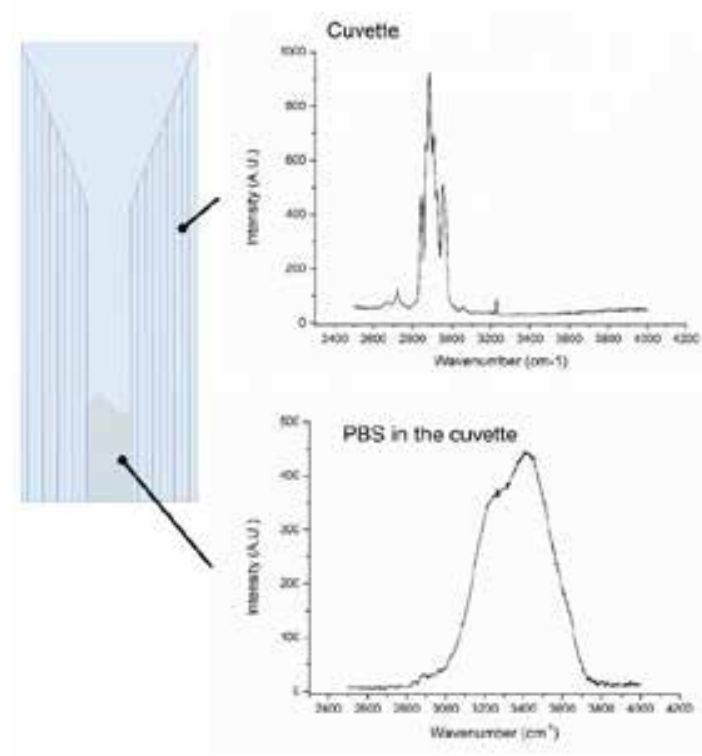

a

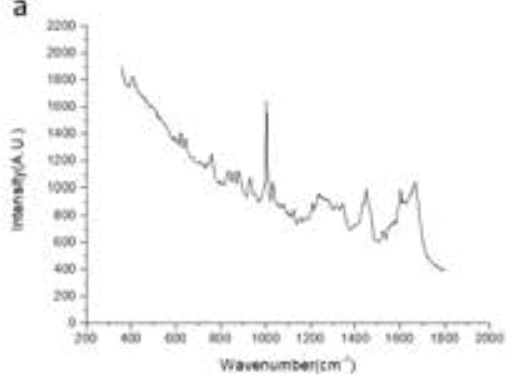

b

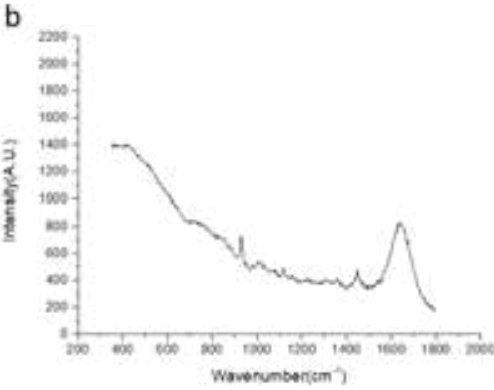

Figure 6b-5. Raman spectrum of aqueous humor samples from, (a) a $0.5 \%$ ketorolac submerged pig eye (3 frames of 60 seconds), and (b) from a rabbit eye (3 frames of 60 seconds on PKXF samples).

\section{6b.2.2 In vitro measurement of enucleated pig eyes}

Fresh domestic pig (Sus Scrofa Domesticus) eyes were obtained from a local abattoir ("Slachthuis Kerkrade Holding", Kerkrade, NL). The enucleated eyes were 
transported to the laboratory on ice and used within 3 hours after enucleation. Before use, the pig eyes were inspected with a stereo microscope (Olympus SZX9, Tokyo, JP). Only eyes with clear corneas without visible corneal damage were used in the experiment. The excess tissues of the eye were removed carefully where after the eyes were washed in phosphate buffered saline (PBS) ( $\mathrm{pH}$ of 7.4). Meanwhile, ketorolac (MSN laboratories, Telangana, IN) was dissolved in PBS creating concentrations of $0.05 \%, 0.1 \%, 0.125 \%, 0.25 \%, 0.5 \%, 1.0 \%, 1.25 \%, 2.5 \%$, and $5.0 \%$. The pig eyes were submerged in $15 \mathrm{~mL}$ of a diluted ketorolac solution. As negative control, PBS was used, and as positive control $0.5 \%$ ketorolac ophthalmic solution (Acular ${ }^{\mathrm{TM}}$, Allergan, Dublin, IR) was used as submerging solution. For each concentration, three eyes were used. Before the Raman measurements, pig eyes were stored in the dark at $4^{\circ} \mathrm{C}$ for 24 hours. Before measurements were taken, the eyes were inserted in a home-designed holder (figure 6b-6).

A long-working-distance microscope objective lens (Jena lens, magnification x 25; numerical aperture $=0.50$; focal length $=10 \mathrm{~mm}$; Carl Zeiss, Jena, DE) was used as focus lens for the Raman system (figure 6b-7a). A f60 lens combined with a Gonio lens (Haag-Streit Meridian, CGA1, Köniz, $\mathrm{CH}$ ) also been used for pig eye measurement (figure 6b-7b). Methoce ${ }^{\circledR} 2 \%$ (OmniVision ,Santa Clara, CA, US) was used to connect the Gonio lens to the cornea. The samples were exposed to 3 frames for 60 seconds. A detailed description can be found in the manuscript from Bertens et al.[1]
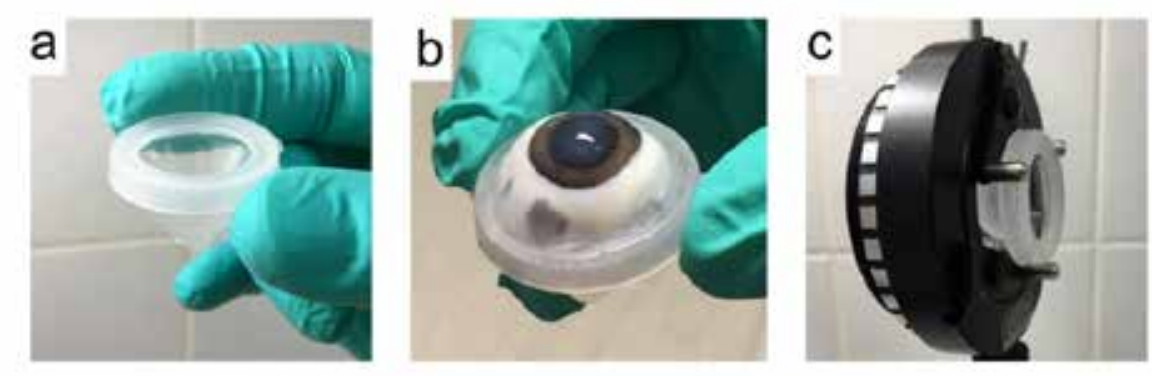

Figure 6b-6. Holder for enucleated eyes. (a) Shows an empty holder, (b) shows a holder with a pig eye, and (c) shows the empty holder on an adjustable lens mount.

\section{6b.2.3 In vivo measurement of the rabbit eyes}

Twelve New Zealand white rabbits (weight ranged from $2.0 \mathrm{~kg}$ to $2.5 \mathrm{~kg}$ upon arrival) were obtained from Envigo (Horst, NL). The rabbits were group housed with 6 animals per cage with males and females separated. The rabbits had ad libitum access to water and food. One week was given to acclimatize before rabbits were used in the experiments. The rabbits were treated with $50 \mu \mathrm{L}$ Acular ${ }^{\mathrm{TM}}$ in the lower conjunctival fornix of their right eye. The contralateral eyes were treated with $50 \mu \mathrm{L}$ 
sterile buffered saline solution (BSS, B. Braun, Melsungen AG, DE manufacturer). Both treatments were performed three times a day. Measurements were taken on day 0 , day 7 , day 14 , day 21 , and day 28 . Four rabbits were used to optimize the system parameters as shown in Table $6 b-2$.

Rabbits were measured using setup as shown in figure $6 b-7 b$. During the examinations, rabbits were anesthetized intramuscularly with ketamine (Alfasan, Woerden, NL) and midazolam (Actavis, Dublin, IR), $50 \mathrm{mg} / \mathrm{kg}$ and $5 \mathrm{mg} / \mathrm{kg}$, respectively. Both eyes of the rabbit were measured by the Raman system. All measurements were performed at random, 1 to 3 hours after receiving the eye drops. Measurement was performed with 30 second exposure times using 2 frames. All animal procedures were conducted according to the ARVO Statement for the Use of Animals in Ophthalmic and Visual Research and the Guidelines of the Central Laboratory Animal Facility of Maastricht University. All protocols were approved by the Central Committee for Animal research and were in accordance with the European Guidelines (2010/63/EU).
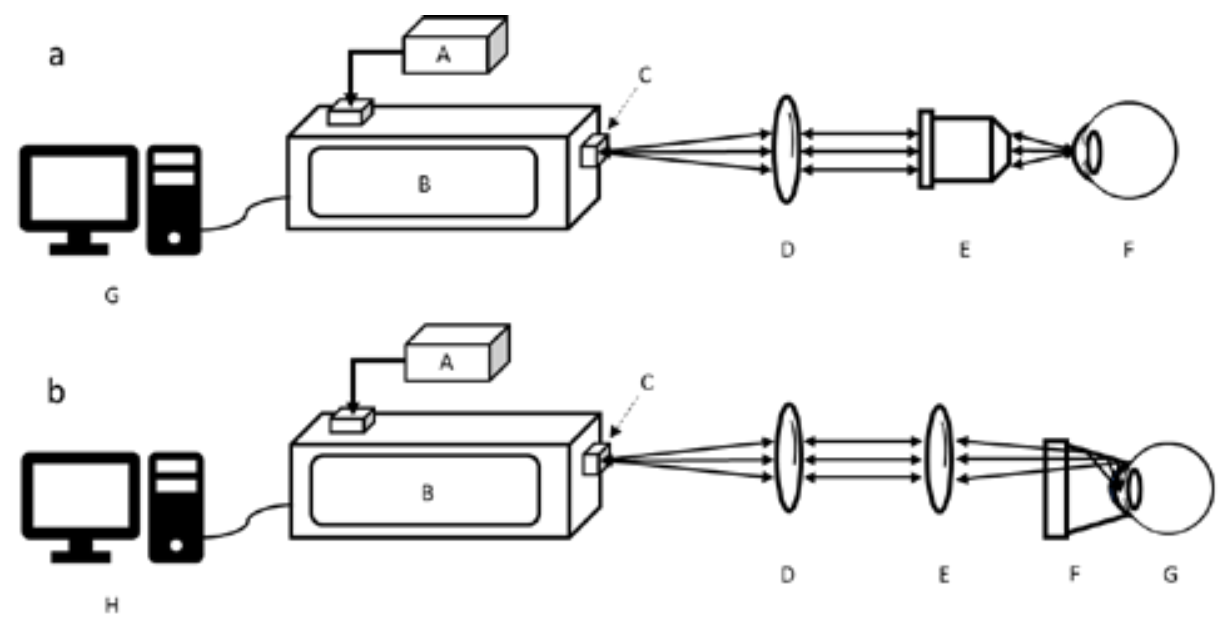

Figure 6b-7. In vitro and in vivo settings of the Raman system.

(a) The set-up is for in vitro pig eye measurements by a Jena lens. (A) laser; (B) Raman module, with (C) $25 \mu \mathrm{m}$ pinhole; (D) collimation f80 lens; (E) objective (Jena lens); (F) pig eye; (G) computer.

(b) The set-up is for in vitro pig eye and in vivo rabbit measurements, a Gonio lens in combination with a f60 focus lens are used for focus in the anterior chamber of the animal eye. (A) laser; (B) Raman module, with (C) $25 \mu \mathrm{m}$ pinhole; (D) collimation f80 lens; (E) f60 lens; (F) a Gonio (one-mirror) lens; (G) pig eye (in vitro) or rabbit eye (in vivo); (H) computer.

Arrows indicate direction of excitation laser light and backscattered Raman light.

\section{6b.2.4 In vitro measurement of the aqueous humor}

For cuvette detection, $50 \mu \mathrm{L}$ to $150 \mu \mathrm{L}$ aqueous humor was obtained from an anterior chamber paracentesis from the eyes using an insulin syringe (BD Micro-Fine ${ }^{\mathrm{TM}}$, 
Becton Dickinson, NJ, US). $50 \mu \mathrm{L}$ was drawn from rabbit eyes after topical sedation (1 drop 0.4\% Oxybuprocaine hydrochloride solution (Bausch \& Lomb Pharma, Brussels, BE)), $100 \mu \mathrm{L}$ to $150 \mu \mathrm{L}$ was drawn from the pig eyes. As a negative control, $100 \mu \mathrm{L}$ aqueous humor was drawn from seven healthy control rabbits within 10 minutes after sacrifice, no topical treatment nor were anaesthetics used.

All aqueous humor samples were frozen on dry ice immediately after sampling and stored in a $-80^{\circ} \mathrm{C}$ freezer until measurements. Samples were measured using a f 80 lens in front of the sample container (figure $6 b-8$ ). The sample was measured for 3 frames in a disposable cuvette (\#7592-00, Sigma-Aldrich, MO, US) with 60 seconds per frame.

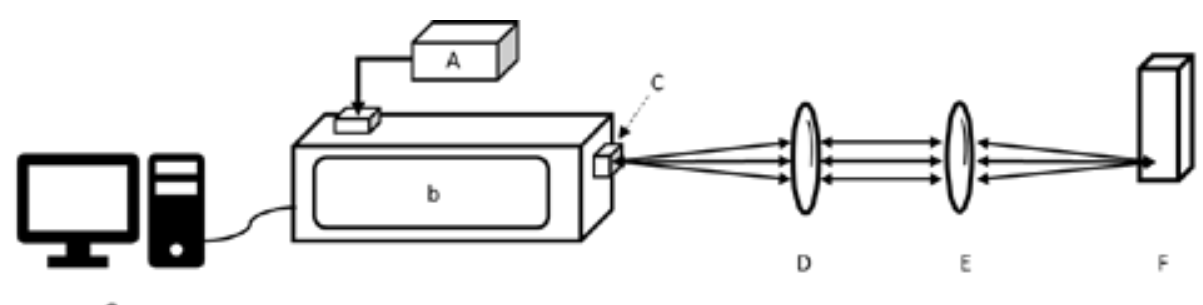

G

Figure 6b-8. In vitro settings of the Raman system with a cuvette. (A) laser; (B) Raman module, with (C) $25 \mu \mathrm{m}$ pinhole; (D) collimation f80 lens; (E) focusing f80 lens (F) samples within cuvette; $(G)$ computer. Arrows indicate direction of excitation laser light and backscattered Raman light

\section{6b.3. References}

1. Bertens, C.J.F., Zhang, S., Erckens, R.J., van den Biggelaar, F., Berendschot, T., Webers, C.A.B., Nuijts, R., and Gijs, M., Confocal Raman spectroscopy: Evaluation of a non-invasive technique for the detection of topically applied ketorolac tromethamine in vitro and in vivo. Int $\mathrm{J}$ Pharm, 2019. 570: p. 118641.

2. Bertens*, C.J.F., Zhang*, S., Erckens, R.J., Biggelaar, F.J.H.M.v.d., Berendschot, T.T.J.M., Webers, C.A.B., Nuijts, R.M.M.A., and Gijs, M., Pipeline for the removal of hardware related artifacts and background noise for Raman spectroscopy. MethodsX, 2019.

3. Treffer, R., Lin, X., Bailo, E., Deckert-Gaudig, T., and Deckert, V., Distinction of nucleobases a tip-enhanced Raman approach. Beilstein J Nanotechnol, 2011. 2: p. 628-637.

4. Erckens, R.J., Jongsma, F.H., Wicksted, J.P., Hendrikse, F., March, W.F., and Motamedi, M., Raman spectroscopy in ophthalmology: from experimental tool to applications in vivo. Lasers Med Sci, 2001. 16(4): p. 236-252.

5. $\quad$ Erckens, R.J., Jongsma, F.H.M., Wicksted, J.P., Hendrikse, F., March, W.F., and Motamedi, M., Drug-induced corneal hydration changes monitored in vivo by non-invasive confocal Raman spectroscopy. Journal of Raman Spectroscopy, 2001. 32(9): p. 733-737.

6. Bot, A.C., Huizinga, A., de Mul, F.F., Vrensen, G.F., and Greve, J., Raman microspectroscopy of fixed rabbit and human lenses and lens slices: new potentialities. Exp Eye Res, 1989. 49(2): p. 161-169.

7. Movasaghi, Z., Rehman, S., and Rehman, I.U., Raman Spectroscopy of Biological Tissues. Applied Spectroscopy Reviews, 2007. 42(5): p. 493-541.

8. Elshout, M., Erckens, R.J., Webers, C.A., Beckers, H.J., Berendschot, T.T., de Brabander, J., Hendrikse, F., and Schouten, J.S., Detection of Raman spectra in ocular drugs for potential in vivo application of Raman spectroscopy. J Ocul Pharmacol Ther, 2011. 27(5): p. 445-451. 
In vitro and in vivo datasets of topically applied ketorolac tromethamine in aqueous humor using Raman spectroscopy 


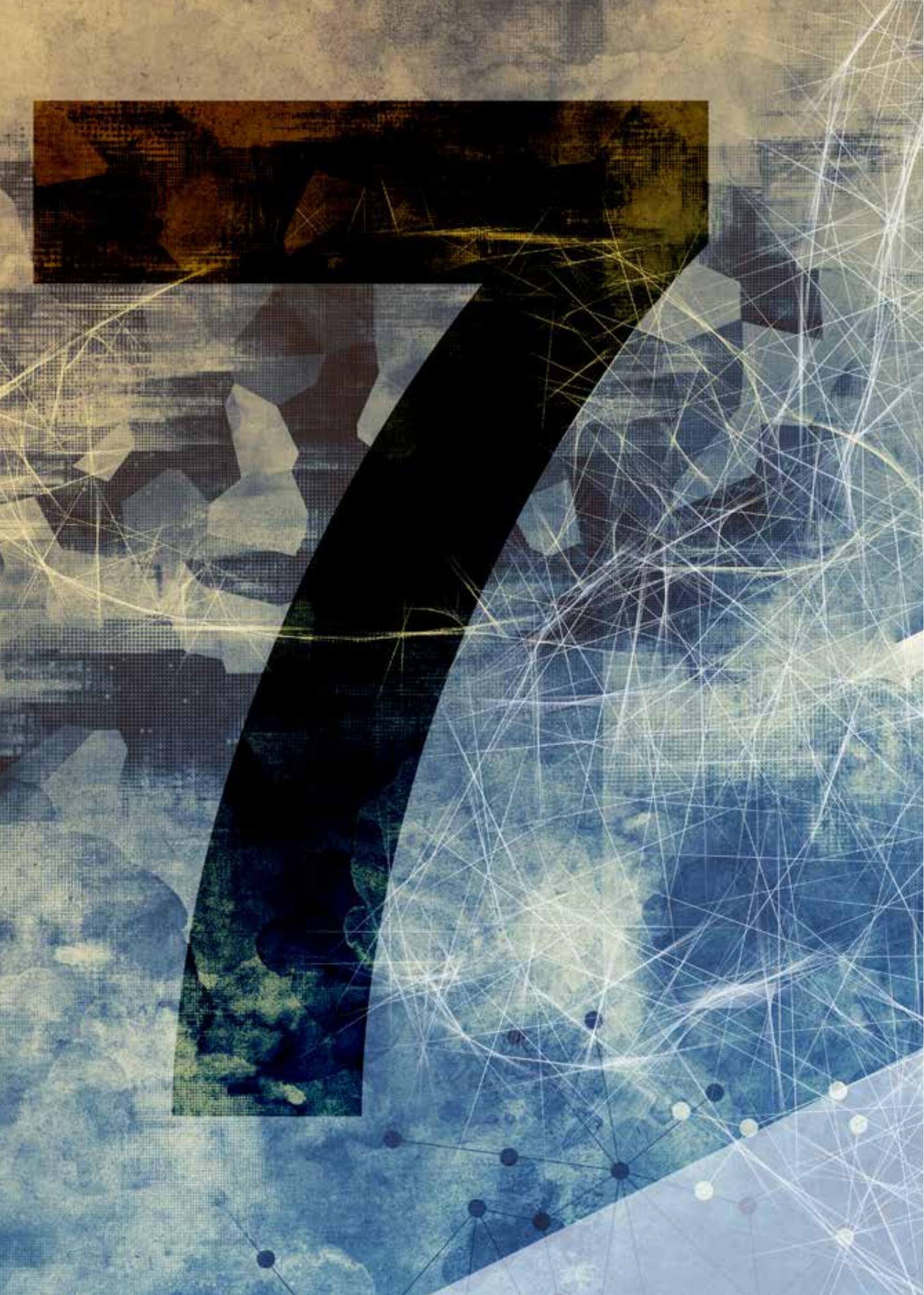





\section{Abstract}

Purpose. To show feasibility of computerized techniques for ocular redness quantification in clinical studies, and to propose an automatic, objective method.

Methods. Software for quantification of redness of the bulbar conjunctiva was developed. It provides an interface for manual and automatic sclera segmentation along with automated alignment of region of interest to enable estimation of changes in redness. The software also includes the redness scoring methods: (i) contrastlimited adaptive histogram equalization (CLAHE) in red-green-blue (RGB) color model, (ii) product of saturation and hue in hue-saturation-value (HSV), and (iii) average of angular sections in HSV. Our validation pipeline compares the scoring outcomes from the perspectives of segmentation reliability, segmentation precision, segmentation automation, and the choice of redness scoring methods.

Results. Ninety-two photographs of eyes before and after provoked redness were evaluated. Redness in manually segmented images was significantly different within human observers (inter-observer, $p=0.04$ ) and two scoring sessions (intra-observer, $p<0.001)$. Automated segmentation showed the smallest variability and, can therefore be seen as a robust segmentation method. The RGB-based scoring method was less sensitive in redness assessment.

Conclusions. Computation of ocular redness depends heavily on sclera segmentation. Manual segmentation appears to be subjective, resulting in systematic errors in intra- and inter-observer settings. At the same time, automatic segmentation seems to be consistent. The scoring methods relying on HSV color space appeared to be more consistent.

Translational relevance. Computerized quantification of ocular redness holds great promise to objectify ocular redness in the standard clinical care and, in particular, in clinical trials. 


\subsection{Introduction}

A wide range of ocular conditions are characterized by bulbar redness including dry eye disease, (allergic) conjunctivitis, blepharitis, corneal abrasion, foreign body, subconjunctival hemorrhage, keratitis, iritis, glaucoma, chemical burn, and scleritis.[1] In addition, ocular redness is often observed in contact lens wearers.[2] Ocular redness is a sign of ocular inflammation and is generally associated with pain or discomfort and often accompanied with vision problems.

Ocular redness is an important diagnostic feature to detect diseases and to monitor disease progression and treatment. In clinical practice, the most common way to grade eye redness relies on the usage of special reference scales. The most known grading scales are the McMonnies/Chapman-Davies scale [2], Efron scale [3], the Institute for Eye Research scale (also known as CCLRU) [4], and the validated bulbar redness scale.[5] Using such techniques, a clinician grades the patient's condition using photographic $[2,4,5]$ or artist-rendered [3] reference images. This method is very simple, and a trained clinician would need about ten seconds in order to accomplish grading. However, these methods also have several major drawbacks. First, the grading is highly subjective since it depends on the knowledge and experience of the clinician. Secondly, due to the limited set of grading states, it cannot provide continuous linear quantitative evaluation, which makes these methods not very sensitive to small changes in ocular redness in early stages of disease. However, this sensitivity is of high importance for early diagnosis and in clinical trials [6], which evaluate the safety of new ophthalmic drugs, drug formulations or drug delivery devices.[7] Furthermore, because of the lack of photographic documentation, grading by this method is not reproducible and does not allow for a second observer. Hence, despite a relatively high number of existing approaches, none of them is regarded as a gold standard.

In the present study, we investigated the reliability of computerized techniques for ocular redness quantification. In particular, we are interested in establishing the reliability of the redness score depending on region of interest (ROI) segmentation and a chosen scoring method. Furthermore, we propose a processing pipeline designed to avoid subjectivity by replacing all human interactions with automated algorithms.

\subsection{Materials and methods}

In order to extract data from ocular photographs, we developed a software tool featuring a graphical user interface (GUI) for sclera selection and segmentation. After image acquisition, we implemented a machine learning method for automatic sclera segmentation, which is independent of image size, eye pose and illumination. Based on the concept of Sárándi et al. [6], a method was developed for the selection 
of the region of interest (ROI). $\mathrm{ROI}$ registration and intersection was performed in corresponding images using feature matching [8], assuring that exactly the same part of the eye is considered for the computation of redness scores over the time. For redness scores, we implemented and compared the approaches of Park et al. [9], Amparo et al. [10], and Sárándi et al.[6] Figure 7-1 illustrates our processing pipeline.

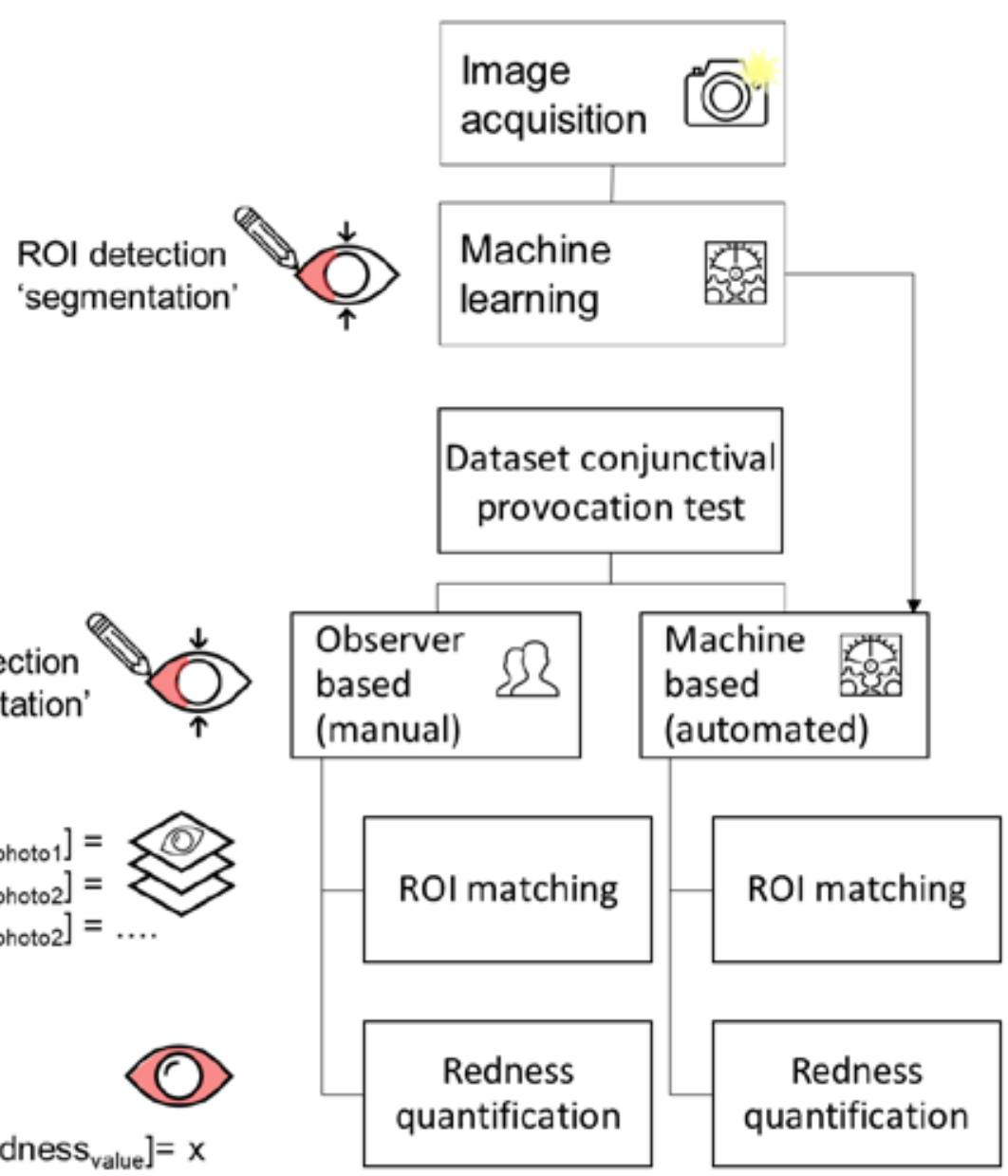

Figure 7-1. Organizational chart of the experiment. ROI, region of interest.

\subsubsection{Image acquisition}

For software development (training of a machine learning classifier) and preliminary testing, a total of 97 photographs of 18 volunteers were taken at the University Eye 
Clinic Maastricht (Maastricht, the Netherlands). The protocol was approved by the local ethics committee and the national authorities. The study procedures were performed in accordance with the tenets of the Declaration of Helsinki. All participants signed written informed consent before inclusion. Three photographs were taken per eye at $6.3 x$ times magnification using a calibrated Haag-Streit BX900 slit-lamp bio-microscope (Haag Streit AG, Bern, Switzerland) in combination with a computer-operated digital camera (Nikon D7100; Nikon, Tokyo, Japan). The volunteers were asked to look left, right, and up. Images were exported as JPG files (2992 x 2000 pixels, $150 \mathrm{dpi}$ ). Background illumination was used on full intensity (100\% open) and grey-filter settings were set to $100 \%$ open. Slit beam illumination was used with a diffusion filter, a width of $15 \mathrm{~mm}$ and $8 \mathrm{~mm}$ height of the beam at a $45^{\circ}$ oblique angle.

For evaluation, the dataset from the conjunctival provocation test [6] was used. The dataset contains 92 images of 23 patients. The images were taken in pairs: before (called 'reference image') and after (called 'response image') the application of an inducing redness allergen. For each patient, the procedure was performed twice in separate visits (visit 1 and visit 2) (figure S7-1). The dataset used was recorded in the controlled environment with the same equipment.

\subsubsection{Automatic ROI detection}

For ROI segmentation, non-parametric models (i.e., random decision forest) were used.[11] For training, we used the open-source machine learning software Weka [12] and the Trainable Weka Segmentation (TWS) toolkit.[13] It utilized a fast (i.e., multithreaded) version of Breiman's random forest algorithm.[14] We initialize with 512 'trees' and eight random features per node. These parameters were derived empirically. Images of eight different subjects were used for training: the subjects feature different eye color and skin tone and level of redness and prominence of vascular structure vary within selected samples. Therefore, two classes of regions were selected manually: sclera and background (figure 7-2). Approximate training time was $10 \mathrm{~s}$ per image on the used hardware (Intel Core i7-2620m processor, 8 GB RAM).

Classification is integrated in our custom software written in Java. Using trained model, grayscale probability maps are created for new images where higher intensities correspond to the regions which most likely belong to the sclera (figure 73a). Simple post-processing involving binary threshold and morphological operators is applied to the probabilistic maps such that the largest area with the highest probability score is identified as the ROI (figure 9-3B). The outer contour of the detected ROI is then processed with Bresenham's line algorithm [15], which smoothens the contour and provides adjustment points, which can be used in the $\mathrm{GUI}$ in order to correct the detected ROI manually if necessary (figure 9-3C). 

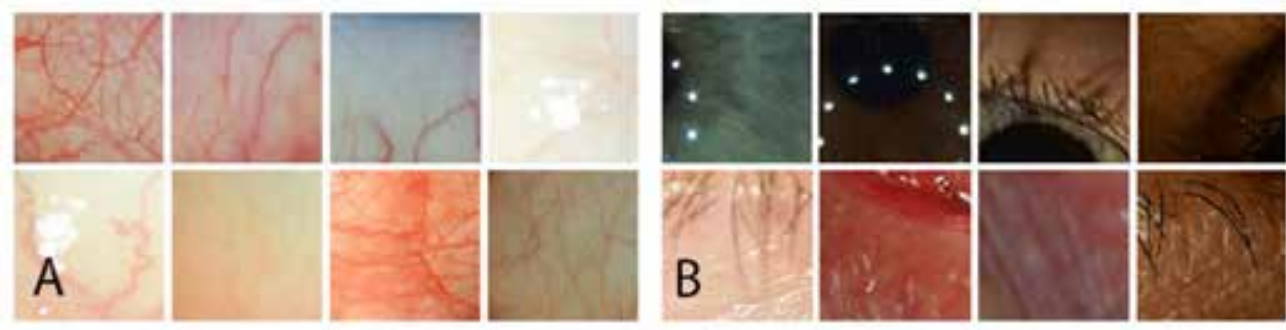

Figure 7-2. Training patches. (A) Example sclera patches. (B) Example non-sclera (background) patches.
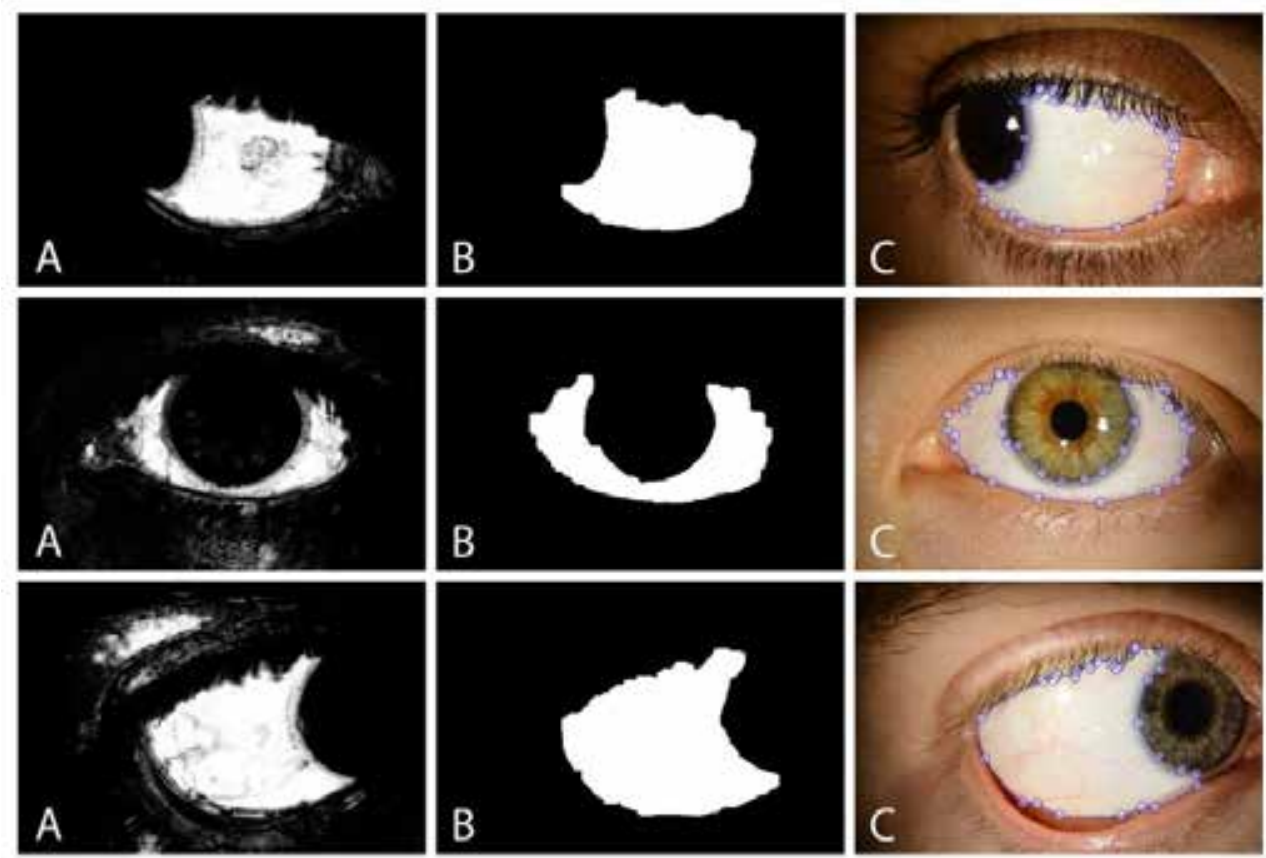

Figure 7-3. Segmentation steps applied to three different subjects: (A) Generated probability map of sclera segmentation: higher intensities correspond to the areas, which most likely belong to the sclera region. (B) $\mathrm{ROI}$ derived out of the probability map using simple thresholding and refinement with morphological operations of erosion and dilation. (C) ROI with adjustment points laid over the original image.

\subsubsection{Manual ROI detection}

Five human observers performed manual segmentation using the GUI interface running the same machine. Four of the human observers performed the segmentation of each image twice. In each manually segmented image pair consisting of the images of the same eye before application of the allergen and after, redness scores were estimated both, before and after applying $\mathrm{ROI}$ matching. 


\subsubsection{ROI matching}

If we want to achieve the most precise comparison between different stages of redness in the same eye, the same parts of the sclera on the photographs need to be measured. It is incorrect to compare redness in two ROIs just after ROI detection because of possible differences in eyelid openness, differences in gaze direction, and also different scales and image resolutions associated with non-standardized acquisition settings. Therefore, we implemented the registration of two or more sequential ROIs to find a common ROI, which shall be used for redness computation. The method is based on detection of landmarks, or points of interest, which are robust to rotation, translation and scale. Scale invariant feature transform (SIFT) points of interest are detected in all ROIs, and point correspondences are estimated by feature similarity.[8] Random sample consensus (RANSAC) is used for robustness refinement.[15] Using these correspondences (figure 7-4A), transformation between the reference and the matched ROIs can be derived and applied to matched ROI. The transformed ROI is laid over the reference ROI, and the intersection of both is used as the common ROI for redness estimation (figure 7$4 B$ ). This is also beneficial for removal of false positives in ROls.
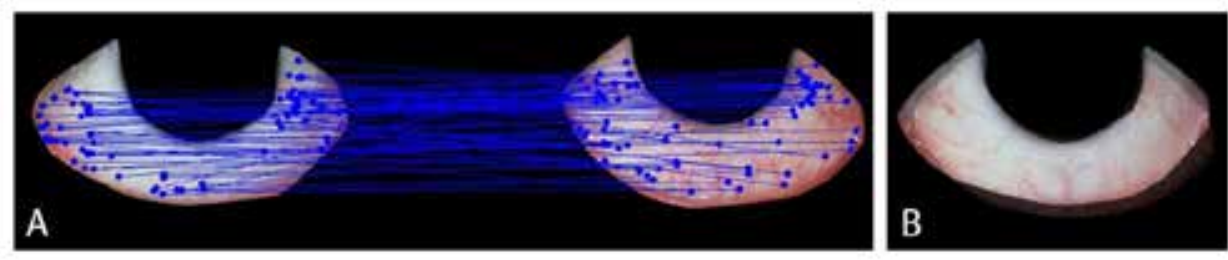

Figure 7-4. (A) Corresponding points of interest are connected with straight lines. Photo on the left was taken before the allergen was applied, and on the right - after. (B) Overlay of registered ROIs: only the overlapping area is considered as ROI for redness computation.

\subsubsection{Redness quantification}

For redness scores, we implemented the approaches of three different studies: Park et al. [9], Amparo et al. [10], and Sárándi et al.[6] (figure 7-5). Park et al. [9] have used the contrast-limited adaptive histogram equalization (CLAHE) for blood vessels enhancement.[16] The vessels are segmented using thresholding and the redness score is calculated as a ratio of number of pixels corresponding to the blood vessels to the total number of pixels in the ROI. Amparo et al.[10] use HSV color space for redness estimation and use the product of saturation and hue mapped to $[0,1]$ interval as the redness score. Sárándi et al.[6] also rely on HSV color space and compute the redness score as an average of maximal values $\max \{0, S, \cos (2 \pi H)\} x$ computed for each pixel in the ROI, where $S$ and $H$ are saturation and hue components of the pixel, respectively. 


\subsubsection{Clinical cases}

To test the final version of the program, three clinical cases of ocular redness were assessed in the University Eye Clinic Maastricht (Maastricht, the Netherlands). Patients signed written informed consent before photos were taken. From both eyes three photos were taken using slit-lamp settings as described in 'Image acquisition'. Patients were asked to glare up, left, and right.
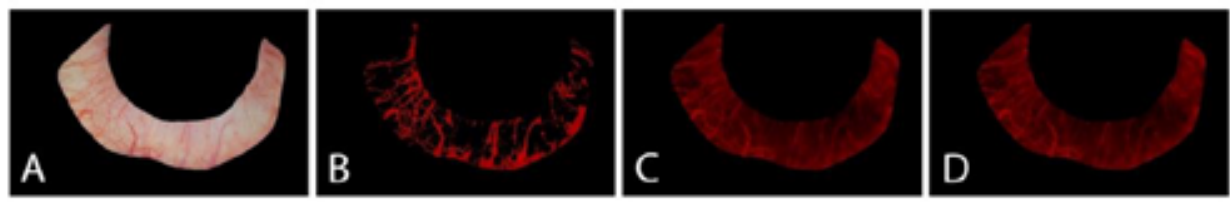

Figure 7-5. (A) ROI selected in the original image. Pixels classified as red using the methods of: (B) Park et al. [9], (C) Amparo et al. [10], (D) Sárándi et al. [6]

\subsubsection{Statistical analysis}

The described system was utilized to determine redness scores computed using three different methods (Park et al. [9], Amparo et al. [10] and Sárándi et al. [6]). First, segmentation reliability, defined as the ability of the observer to produce similar results time after time, also known as intra-observer difference, was evaluated using a test-retest fashion (Bland and Altman plot). To estimate the significance level of difference in redness scores within test and retest segmentations, mean reference and response redness values of both visits were compared using a paired t-test and a general linear model repeated measures test. To exclude the effect of other features, no ROI matching was performed and the score was computed.[6] Second, segmentation precision was defined as inter-observer difference. For that, the mean redness values of the reference recordings in the first visit, using test only (the first segmentation by five human observers), were compared using a general linear model repeated measures test. Again, no ROI matching was performed and the score was computed.

To estimate the robustness of a computer-based method, we evaluated the effect of segmentation automation by comparing the differences between visit 1 and visit 2 of the reference images between values computed with and without ROI matching using analysis of variance (ANOVA). In addition, to prove the assumption that if we include ROI registration, the absolute redness values indicating changes in redness are supposed to be more robust, we computed the scores with and without applying the proposed technique.

We implemented three redness scoring methods and, based on the assumption that a large difference in redness between reference and response shall indicate higher sensitivity, we compared redness differences between reference and response 
values estimated by all three methods (Park et al. [9], Amparo et al. [10] and Sárándi et al. [6]) using automatic segmentations provided by our machine learning method (without ROI matching).

In order to illustrate the clinical applicability by case, we selected three trial subjects from the conjunctival provocation test panel. Based on the subjective assessment on visual differences between the reference image and response image, these subjects were labelled as strong, mild or no responders to the provocation test.

All data is analyzed using SPSS (version 25 IBM, Armonk, NY, US), data is shown as mean \pm standard deviation (SD).

\subsection{Results}

\subsubsection{Segmentation reproducibility}

There was a significant difference $(p<0.001)$ between the test and retest for three out of four human observers (figure 7-6A), meaning that there was a systematic error for the three observers. Further, these systematic errors differed between the observers $(p<0.001)$. Frequency distributions of differences in redness scores between test and retest observations (figure 7-6B) indicate that segmentation by observers 1 and 4 systematically results in larger redness values during the retest ('over-segmentation'), while segmentation by observer 2 systematically provides smaller redness values ('under-segmentation'). Observer 3 is consistent in his manual segmentation. Additionally, observers 2 and 4 display a broad variability in redness values in contrast to observers 1 and 3 . These trends are illustrated by two case examples of 'over-' and 'under-segmentation' and by their mean values of redness difference. Figure 7-7 shows the differences between test and retest versus the mean grading estimate. There is no general relation between the differences and the means, indicating that segmentation reliability is unaffected by the redness score itself. Again, observer 3 shows the best segmentation reliability as a tighter cluster of redness differences around zero can be recognized, while more values falling far from the mean are seen for observers 1,2 , and 4 .

\subsubsection{Segmentation accuracy}

The inter-observer difference, i.e. the difference between multiple human observers for the reference images, was significantly different between the five observers $(p=0.040)$ (figure 7-8A) meaning that manual segmentation is easily affected by subjective factors (figure $7-8 B$ ).

\subsubsection{Segmentation automation}

The overall mean redness difference of the human observers showed an increase by implementing ROI matching, however insignificant (figures 7-9A and 7-10). This 
is illustrated by two case examples segmented by observer 4 that shows an increase in redness difference after implementation of ROI matching (figure 7-9B). With the machine learning approach $\mathrm{ROI}$ matching improved the results as the mean redness difference became smaller, though insignificant as well.
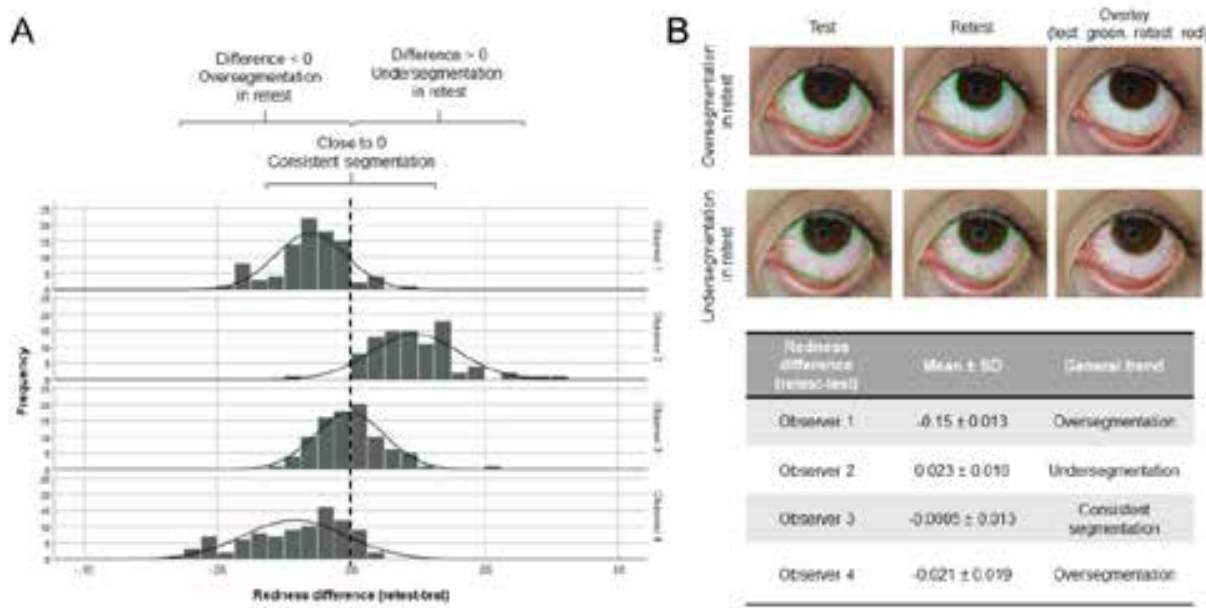

Figure 7-6. (A) Frequency distributions of redness differences between test and retest observations for four human observers. (B) Example of test and retest with an overlay from an over-segmentation and an under-segmentation. The table shows an overview of the general trend from the observers.

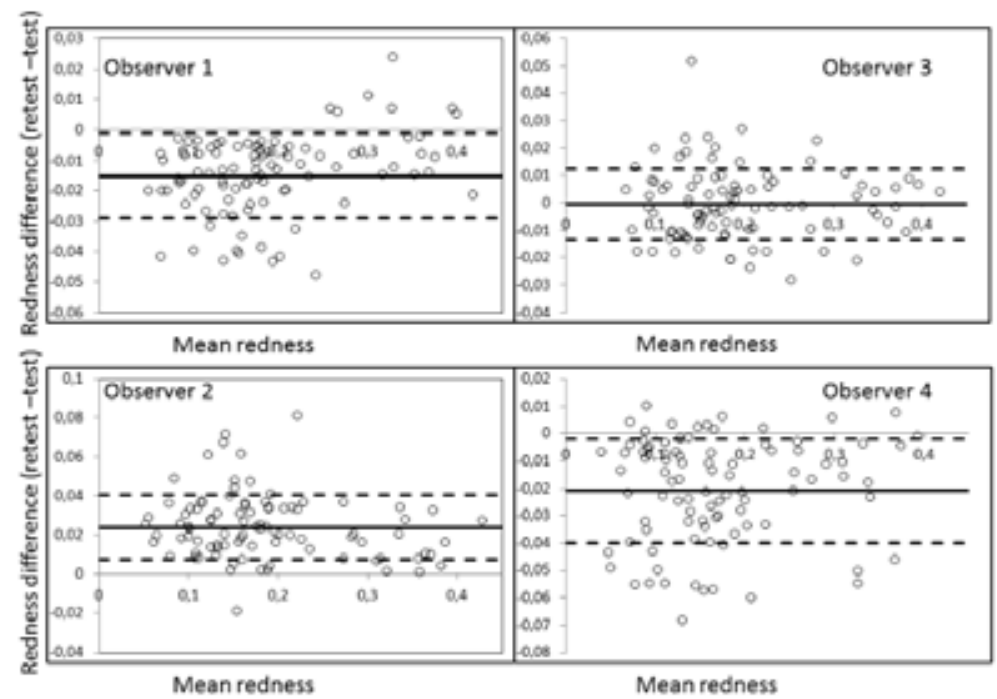

Figure 7-7. Redness difference versus mean redness of test and retest redness values for four human observers. The thick solid line represent the mean value of test-retest discrepancies and the dotted lines represent the mean \pm standard deviation. 


\subsubsection{Redness scoring method}

Figure 7-11 shows that the redness values calculated by the method of Park et al. [9] largely overlap and, thus, is insufficiently able to detect differences in redness. In contrast, little overlap can be observed at the methods by Amparo et al. [10] and Sárándi et al.[6] The sensitivities of these two methods are similar. Three case examples illustrate that the method of Park et al. [9] is insensitive to detect differences in redness for the strong and mild responder, while the sensitivities of Amparo et al. [10] and Sárándi et al. [6] are comparable (figure 7-12).

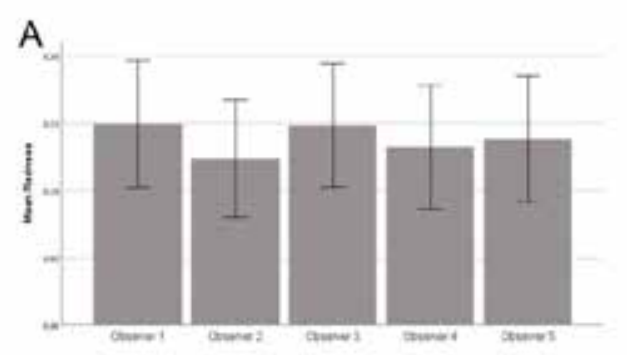

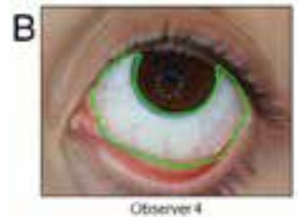

Otrevere 4
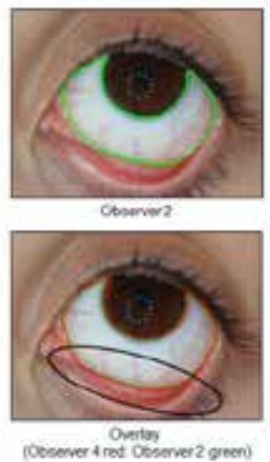

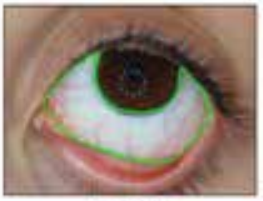

obiene?
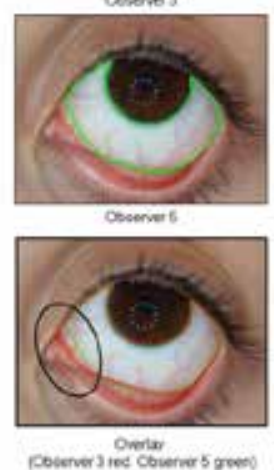

Figure 7-8. (A) Mean redness values ( \pm standard deviation) of the reference recordings in the first visit, using test only, without ROI matching, computed using the method of Sárándi et al. [6] for five observers.

(B) Differences between observers related to the conjunctival border (left column) and the semilunar conjunctival fold (right column).

\subsubsection{Clinical application by case}

Our automated tool generated nominal values of redness difference between the reference (before) and response (after) images (figure 7-13). Although the subjective assessment in these simplistic examples is straightforward, one can appreciate the sensitivity of our automated tool, with up to nine-fold differences in redness difference between two cases of the same participant.

When no follow-up visit is available, redness can be scored using the contralateral eye as shown in figure 7-14A. Three clinical cases are tested using the methods by Park et al. [9], Amparo et al. [10] and Sárándi et al.[6] (figure 7-14B). In all methods the affected eye provides a higher redness value compared to the contralateral eye. The values generated by the methods of Amparo et al. [10] and Sárándi et al. [6] are almost two times higher in intensity compared to the values generated by the method 
of Park et al.[9]

\subsection{Discussion and conclusion}

Ocular redness is an observable clinical response of the ocular surface in pathological conditions. To some extent, the degree of redness may reflect the severity of the disease. In this context, quantification of ocular redness can be of use in both clinical and research settings. Examples of conditions which are often associated with ocular redness are dry eyes disease, contact lens complications and allergic conjunctivitis. In clinical practice, sensitive quantification of ocular redness would allow to stage the (subclinical) disease, to monitor progression of the disease and to control and regulate treatment efficacy.

A

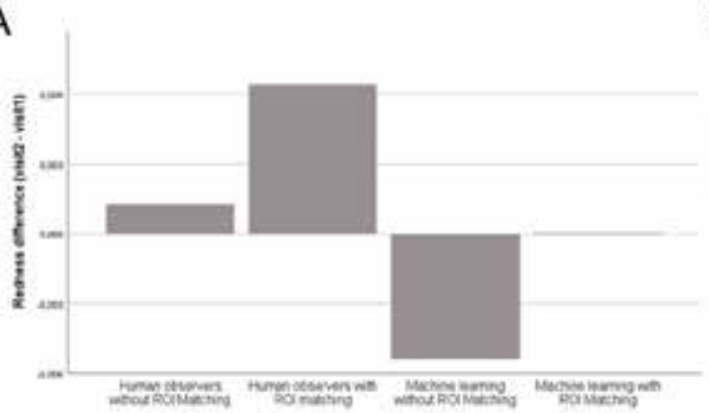

B

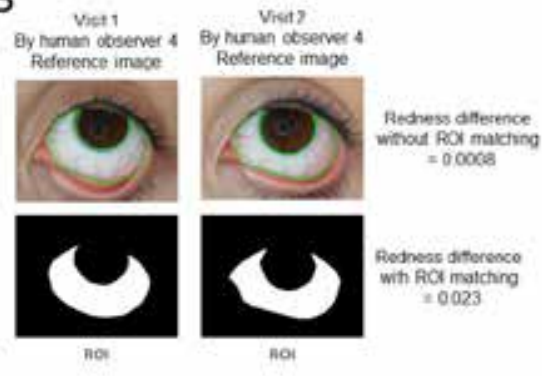

Figure 7-9. (A) Mean redness differences between visit 1 and visit 2 of the reference images for all human observers and the machine-learning approach, both with and without ROI-matching. (B) Example of Redness difference with or without ROI matching.

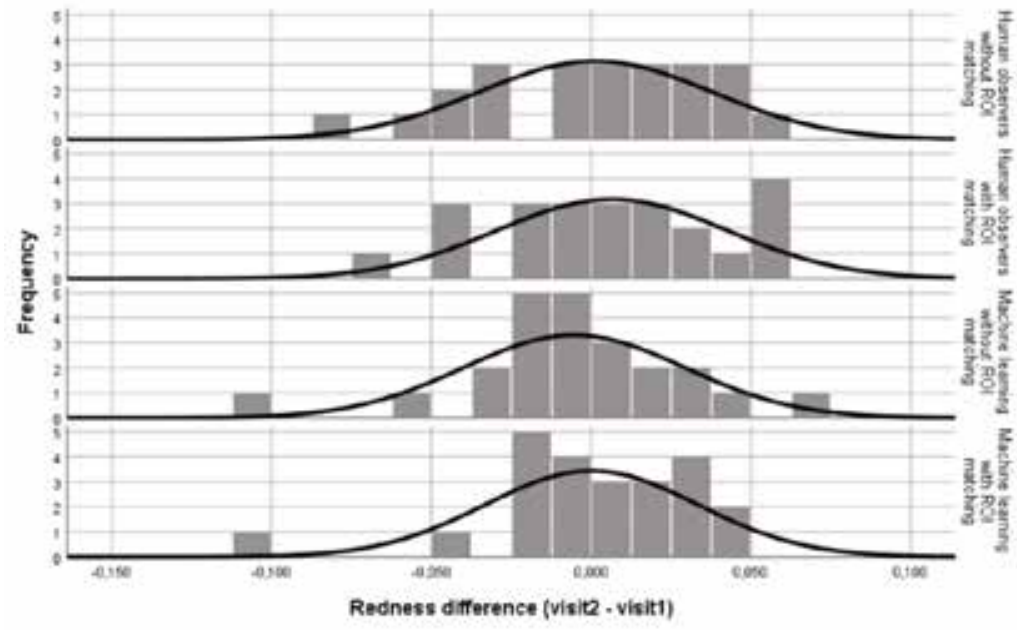

Figure 7-10. Frequency distribution of the redness differences between visit 1 and visit 2 for all human observers and the machine-learning approach, both without and with ROI-matching. 


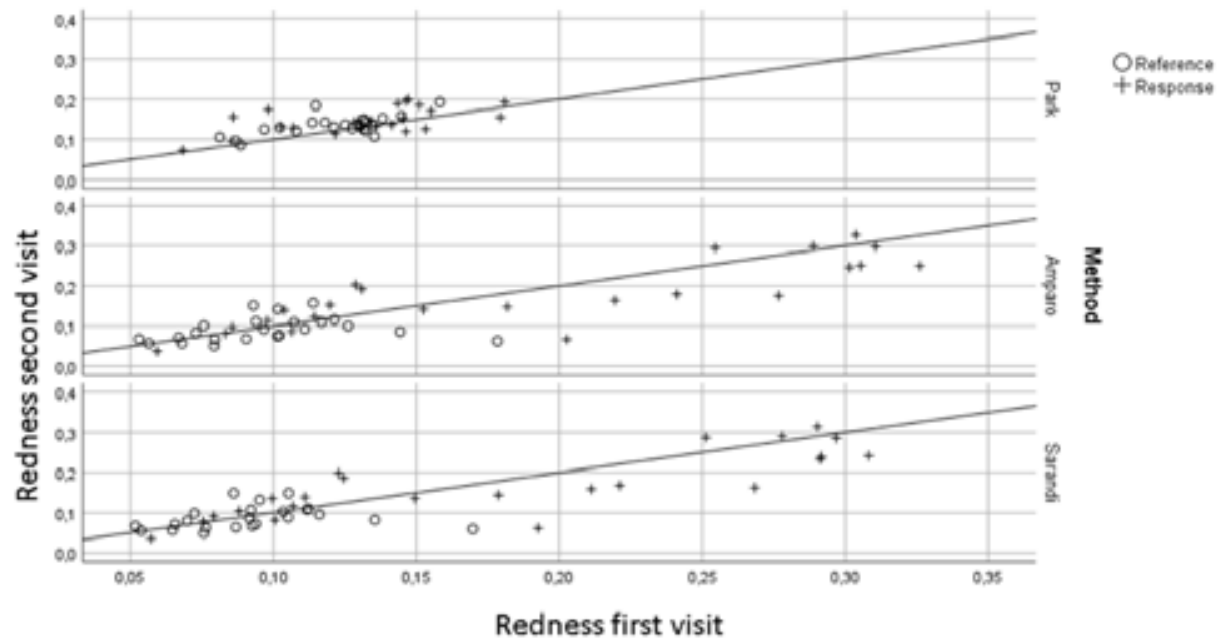

Figure 7-11. Comparison of redness scores for the machine-learning approach of three different redness scoring methods without ROI matching. The solid line shows the equality.
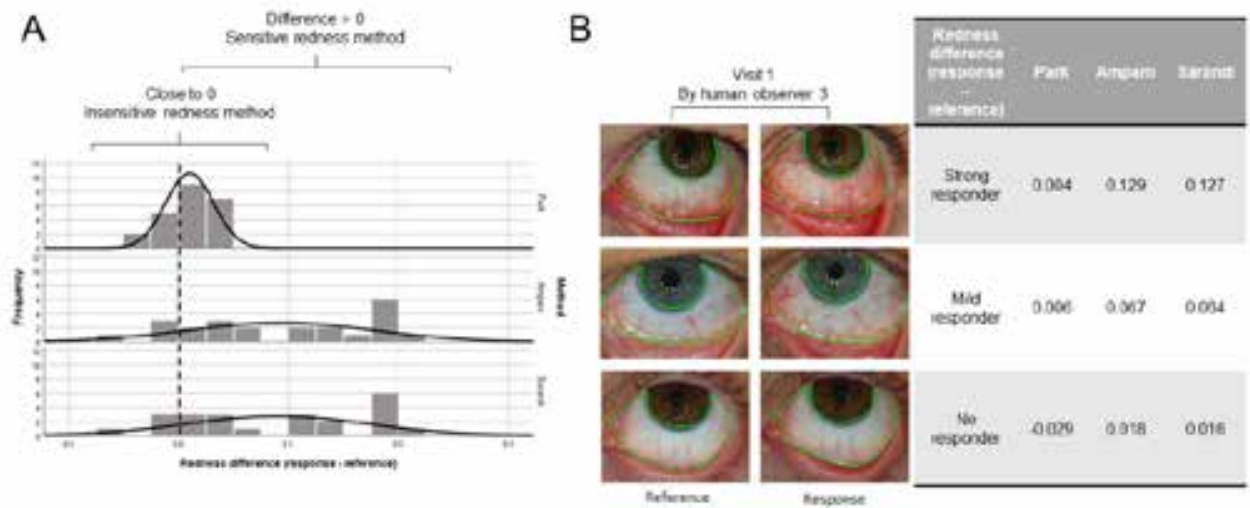

Figure 7-12. (A) Frequency distribution of the redness differences between the reference and response through three different redness scoring methods. (B) Illustrated example between a strong responder, mild responder, and a no responder and the values provided through the three different redness scoring methods.

Another application for computerized quantification of ocular redness would be in a setting of multicenter clinical trial to investigate the safety of new topical drugs or devices with regards to undesired side-effects such as eye itching, reddening, or tearing. Self-assessment questionnaires are usually filled in by study subjects in order to evaluate the level of discomfort, while redness and changes in its level are assessed by clinicians using the reference scales like the Efron scale or VBR. We believe that using an automated tool would increase the objectivity of such a study due to elimination of inter- and intra-observer variability. 


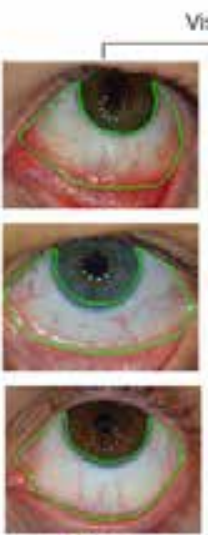

Reference
Visit 1
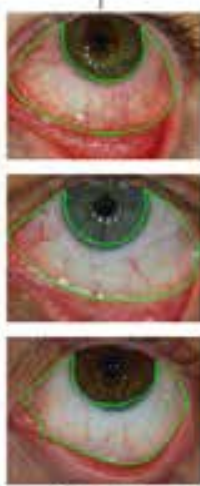

Response
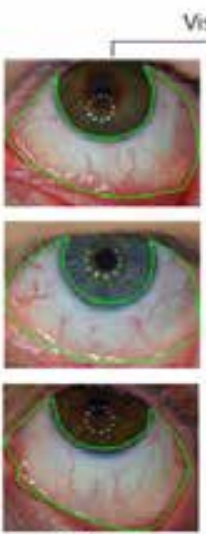

Reference

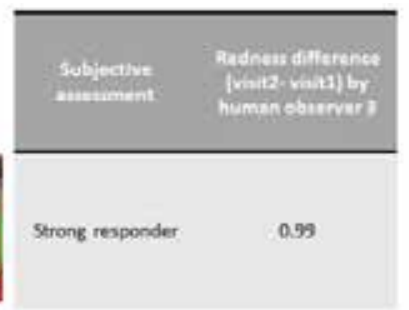

Mild responder $\quad 0.75$

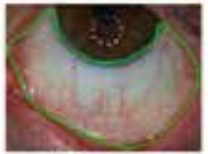

Response

Figure 7-13. Clinical application of the automated software by case examples in a conjunctival provocation test.
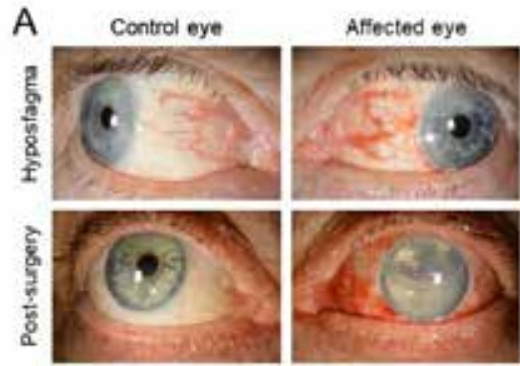

B
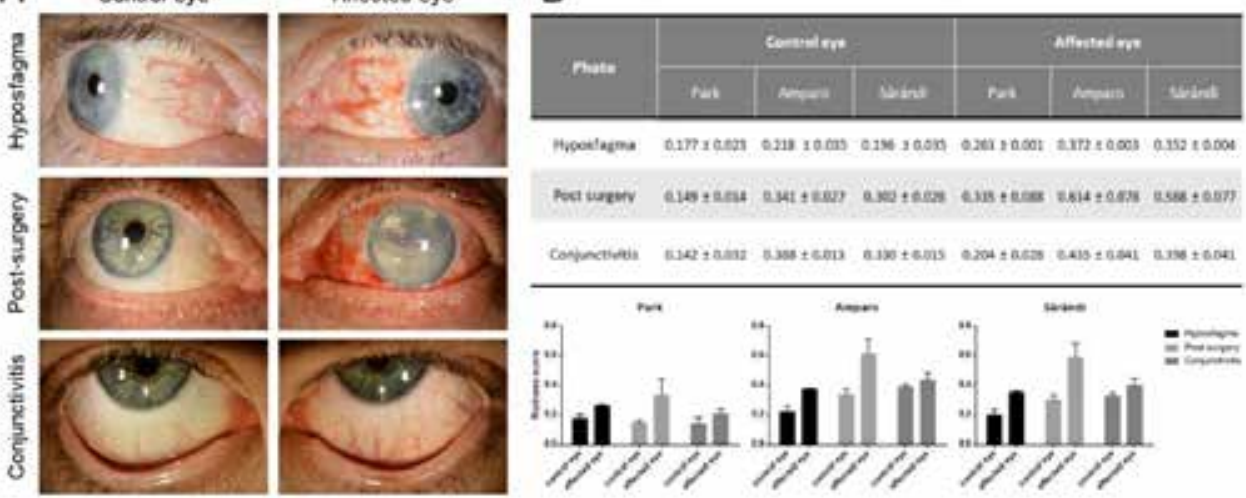

Figure 7-14. Three cases of ocular redness from the clinic. (A) A hyposfagma, post-surgical redness, and a mild form of conjunctivitis. (B) The table shows the numeric redness values of three pictures, averaged \pm SD with visualization as bar graphs below the table.

At the end of the last century, several researchers tried to objectivize ocular redness grading using photographic documentation. In 1990, Kjærgaard et al. presented an experimental pipeline, in which five physicians used a descriptive scale in order to evaluate changes in ocular redness stimulated by the conjunctival provocation test.[17] The final redness values were derived using statistics. The authors claimed a better sensitivity of their method as compared to traditional clinical observations. However, their method still is subjective, requires more resources (man-power) and does not support absolute measurements. 
A further step towards objective quantification of ocular redness was the application of image processing to the photographic images. Such methods rely on machinebased quantification of integral redness of the scleral region [6,10,18-25], blood vessels dilation [9,18,20-23,26-30], and degree of vascular branching [31,32], or combination of these features. Integral redness is usually quantified as a ratio of pixels classified as red to the selected $\mathrm{ROI}[18,19,22,23]$ or as a result of arithmetical operations on color channels in different color models.[6,10,20,21,24,25] Blood vessels are usually segmented using edge detection $[9,21-23,25,26]$, thresholding with a prior enhancement $[9,18,20,28-30]$ or clustering [9] and are described in terms of percentage of vessel coverage $[9,18,20,21,23,25,27,29]$, vessel width $[20,27,30]$, relative redness of vessels [20], and number of vessel segments $[20,30]$. Vascular branching is described using fractal analysis.[31,32]

Diseases and conditions may affect different regions of sclera [30]; it is beneficial to include in the ROI as much of sclera as possible. Fieguth and Simpson [21] postulated that automatic detection of sclera shall be straightforward, because its color is distinct from its surroundings. However, simple color thresholding fails in most of our images. The presence of shadows, light reflections or excessively dilated blood vessels make it hard to distinguish between the sclera and surrounding regions. In contrast to the approaches using manual interaction for ROI detection $[9,10,20,21,25,31]$ or color-based segmentation [6], we therefore use texture information for automated sclera detection.

Sárándi et al. [6] proposed a fully-automated scleral segmentation involving circular Hough transform [33] for iris subtraction and a combination of edge detection and thresholding in YUV color space for sclera localization. Their method works well if the sclera is evenly illuminated and highly distinguishable from the eyelid, but shadows or light reflections on the eyelid or the surrounding skin make the detection error prone. Furthermore, a high concentration of red blood vessels in the sclera often yields a segmentation failure.

It is still worth mentioning that according to visual inspection there are outliers in our segmentation results which may undermine the stability of the general segmentation score. Erroneous ROI detections can be caused by a low quality of a photograph (non-sharp focus, uneven light, reflections) or by a similarity in textures. Blurred edges lead to loss of texture, which makes the detection of ROI and blood vessels not straightforward. The best way to deal with this problem is to control acquisition settings i.e. choosing the smallest aperture. In addition, we provided a customary tool for manual correction of the detected ROI, which still allows usage of images of lesser quality.

Another interesting observation was made with the respect to the provocation test: as it can be seen in figure 7-10 for the response case, the redness in the second visit is lower than the redness in the first visit. We believe that this indicates that the 
provocation is better tolerated by the study subjects upon the second visit. When we used clinical cases the methods from Amparo et al. [10] and Sárándi et al. provided a higher redness value for red eyes compared to the method of Park et al.[9] However, in all cases the methods showed higher signal for the affected eye. This indicates that using the contralateral eye as reference could be a proper solution when no follow-up visits are planned.

Almost all of the existing methods depend on a particular acquisition setup: all images shall be recorded with the same camera and illumination settings. However, this is not always possible, especially when comparing and analyzing a large amount of photographs taken in different laboratories (multi-center studies) or over various periods of time. Amparo et al. [10] introduced semi-automatic white balance correction using the Von Kries approach.[34] However, to our knowledge, full color normalization was not used before for ocular redness assessment. We will investigate this in the future.

In recent years, deep convolutional neural networks (CNN) [35] have gained their popularity in tasks of semantic image segmentation. Such techniques are able to classify the regions not only on a pixel level, but also on the object's shape as contextual information. Since the visible part of human sclera has a distinctive shape, we believe that it is possible to train such a classifier, which would enable recognition of human sclera with a considerably higher accuracy. We are planning to address this in our future work.

In summary, our study demonstrates that interactive user-guided segmentation leads to inconsistency in ocular redness scores driven by both intra-observer and inter-observer variability. As an approach to this problem automatic segmentation can be used. In the current study, we trained a simple random decision forest classifier, which in combination with an automatic ROI matching provided consistent results. Furthermore, our study has shown that the HSV color space resembling human color perception is better suited for redness scoring as it does not depend on illumination and hand-crafted parameters. The outcomes of our proof of concept study are helpful for performing clinical trials targeted to assess ocular redness quantification over time.

\subsection{References}

1. Cronau, H., Kankanala, R.R., and Mauger, T., Diagnosis and management of red eye in primary care. Am Fam Physician, 2010. 81(2): p. 137-144.

2. McMonnies, C.W. and Chapman-Davies, A., Assessment of conjunctival hyperemia in contact lens wearers. Am J Optom Physiol Op, 1987. 64(4): p. 246-250.

3. Efron, N., Clinical application of grading scales for contact lens complications. Optician, 1997. 213(5604): p. 26-34.

4. Institute for Eye Research. IER grading scales, http://www.ier.org.aul, (accessed 18 Jan 2018), online. 
5. Schulze, M.M., Jones, D.A., and Simpson, T.L., The development of validated bulbar redness grading scales. Optom Vis Sci, 2007. 84(10): p. 976-983.

6. Sárándi, I., Claßen, D.P., Astvatsatourov, A., Pfaar, O., Klimek, L., Mösges , R., and Deserno, T.M., Quantitative conjunctival provocation test for controlled clinical trials. Meth Inf Med, 2014. 53(4): p. 238-244.

7. Bertens, C.J.F., Gijs, M., van den Biggelaar, F., and Nuijts, R., Topical drug delivery devices: A review. Exp Eye Res, 2018. 168: p. 149-160.

8. Lowe, D.G., Distinctive image features from scale-invariant keypoints. Int J Comp Vis, 2004. 60(2): p. 91-110.

9. $\quad$ Park, I.K., Chun, Y.S., Kim, K.G., Yang, H.K., and Hwang, J.M., New clinical grading scales and objective measurement for conjunctival injection. Invest Ophthalmol Vis Sci, 2013. 54(8): p. 5249-5257.

10. Amparo, F., Wang, H., Emami-Naeini, P., Karimian, P., and Dana, R., The ocular redness index: a novel automated method for measuring ocular injection. Invest Ophthalmol Vis Sci, 2013. 54(7): p. 4821-4826.

11. Ho, T.K., Random decision forests, in Document Analysis and Recognition. 1995, IEEE: Montreal, Que., Canada. p. 278-282.

12. Witten, I.H., Frank, E., Hall, M.A., and Pal, C.J., Data Mining: Practical Machine Learning Tools and Techniques. 4 ed. Data Management Systems. 2016, Cambridge, MA, USA: Morgan Kaufmann.

13. Arganda-Carreras, I., Kaynig, V., Rueden, C., Eliceiri, K.W., Schindelin, J., Cardona, A., and Sebastian Seung, H., Trainable Weka Segmentation: a machine learning tool for microscopy pixel classification. Bioinform, 2017. 33(15): p. 2424-2426.

14. Breiman, L., Random forests. Mach Learn, 2001. 45(1): p. 5-32.

15. Fischler, M.A. and Bolles, R.C., Random sample consensus: a paradigm for model fitting with applications to image analysis and automated cartography. Comm ACM, 1981. 24(6): p. 381395.

16. Zuiderveld, K., Contrast limited adaptive histogram equalization, in Graphics gems IV, P. Heckbert, Editor. 1994, Academic Press Professional, Inc.: San Diego, CA, USA. p. 474-485.

17. Kjærgaard, S.K., Pedersen, O.F., Taudorf, E., and Mølhave, L., Assessment of changes in eye redness by a photographic method and the relation to sensory eye irritation. Int Arch Occup Env Health, 1990. 62(2): p. 133-137.

18. Willingham, F.F., Cohen, K.L., Coggins, J.M., Tripoli, N.K., Ogle, J.W., and Goldstein, G.M., Automatic quantitative measurement of ocular hyperemia. Curr Eye Res, 1995. 14(12): p. 1101-1108.

19. Horak, F., Bergera, U., Menapace, R., and Schuster, N., Quantification of conjunctival vascular reaction by digital imaging. J Allerg Clin Immun, 1996. 98(3): p. 495-500.

20. Papas, E.B., Key factors in the subjective and objective assessment of conjunctival erythema. Invest Ophthalmol Vis Sci, 2000. 41(3): p. 687-691.

21. Fieguth, P. and Simpson, T., Automated measurement of bulbar redness. Invest Ophthalmol Vis Sci, 2002. 43(2): p. 340-347.

22. Wolffsohn, J.S. and C, P., Clinical monitoring of ocular physiology using digital image analysis. Cont Lens Anterior Eye, 2003. 26(1): p. 27-35.

23. Peterson, R.C. and Wolffsohn, J.S., Sensitivity and reliability of objective image analysis compared to subjective grading of bulbar hyperaemia. Brit J Ophtalmol, 2007. 91(11): p. 14641466.

24. Rodriguez, J.D., Johnston, P.R., Ousler GW, I.I.I., Smith, L.M., and Abelson, M.B., Automated grading system for evaluation of ocular redness associated with dry eye. Clin Ophthalmol, 2013. 7: p. 1197-1204.

25. Ferrari, G., Rabiolo, A., Bignami, F., Sizzano, F., Palini, A., Villa, C., and Rama, P., Quantifying ocular surface inflammation and correlating it with inflammatory cell infiltration in vivo: a novel method. Invest Ophthalmol Vis Sci, 2015. 56(12): p. 7067-7075.

26. Villumsen, J., Ringquist, J., and Alm, A., Image analysis of conjunctival hyperemi. Acta Ophthalm, 1991. 69(4): p. 536-539.

27. Guillon, M. and Shah, D., Objective measurement of contact lens-induced conjunctival redness. Optom Vis Sci, 1996. 73(9): p. 595-605.

28. Owen, C.G., Fitzke, F.W., and Woodward, E.G., A new computer assisted objective method for quantifying vascular changes of the bulbar conjunctivae. Ophthalm Physiol Optics, 1996. 16(5): p. $430-437$. 
29. Dogan, S., Astvatsatourov, A., Deserno, T.M., Bock, F., Shah-Hosseini, K., Michels, A., and Mösges, R., Objectifying the conjunctival provocation test: photography-based rating and digital analysis. Int Arch Allerg Immun, 2014. 163(1): p. 59-68.

30. Zhao, W.J., Duan, F., Li, Z.T., Yang, H.J., Huang, Q., and Wu, K.L., Evaluation of regional bulbar redness using an image-based objective method. Int J Ophtalmol, 2014. 7(1): p. 71-76.

31. Schulze, M.M., Hutchings, N., and Simpson, T.L., The use of fractal analysis and photometry to estimate the accuracy of bulbar redness grading scales. Invest Ophthalmol Vis Sci, 2008. 49(4): p. 1398-1406.

32. Yoneda, T., Sumi, T., Takahashi, A., Hoshikawa, Y., Kobayashi, M., and Fukushima, A., Automated hyperemia analysis software: reliability and reproducibility in healthy subjects. Jap J Ophthalmol, 2012. 56(1): p. 1-7.

33. Lehmann, T.M., Kaup, A., Effert, R., and Meyer-Ebrecht, D., Automatic strabometry by Houghtransformation and covarience-filtering, in ICIP. 1994, IEEE: Los Alamitos, CA, USA. p. 421425.

34. Chong, H.Y., Gortler, S.J., and Zickler, T., The von Kries hypothesis and a basis for color constancy, in ICCV. 2007, IEEE: Rio de Janeiro, Brazil. p. 1-8.

35. LeCun, Y., Bengio, Y., and Hinton, G., Deep learning. Nature, 2015. 521(7553): p. 436. 


\subsection{Supplementary figures}

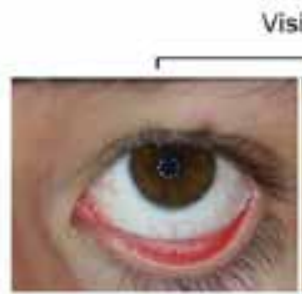

Reference image
Visit 1

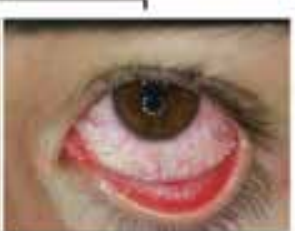

Response image

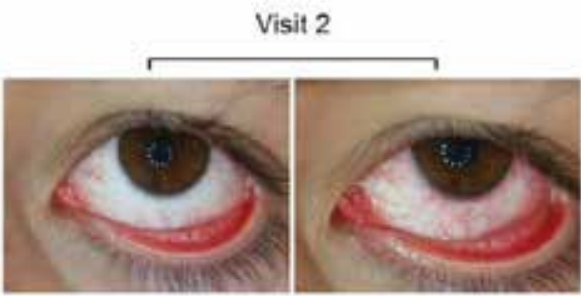

Reference image
Response image

Figure S7-1. Illustration of the collected images per patient. 


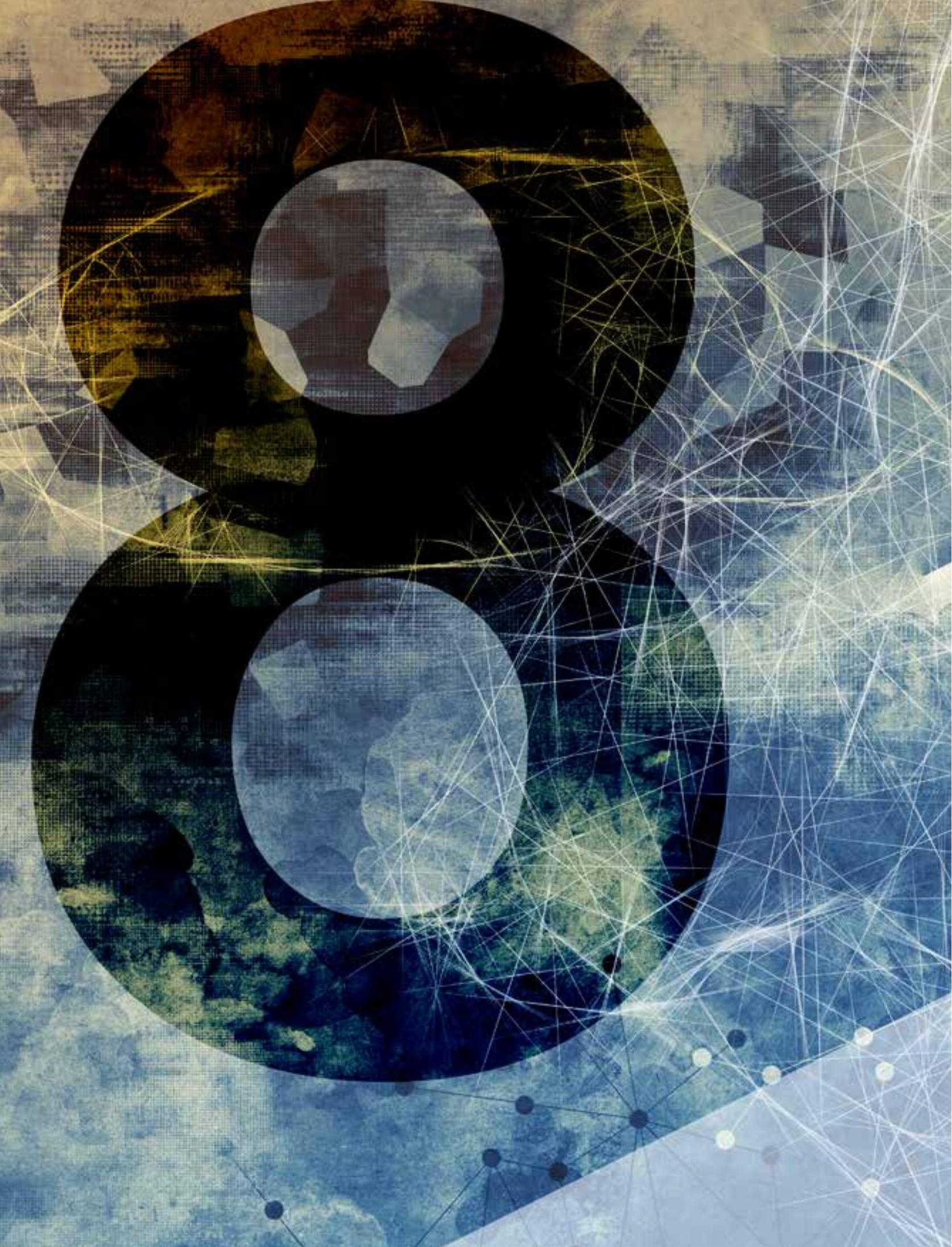




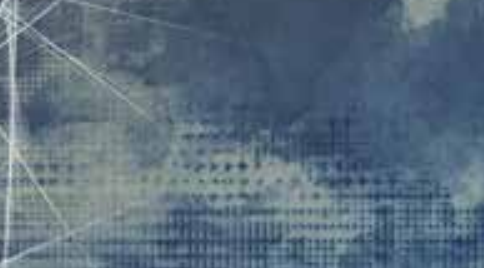

chapter 8.

\section{General discussion}

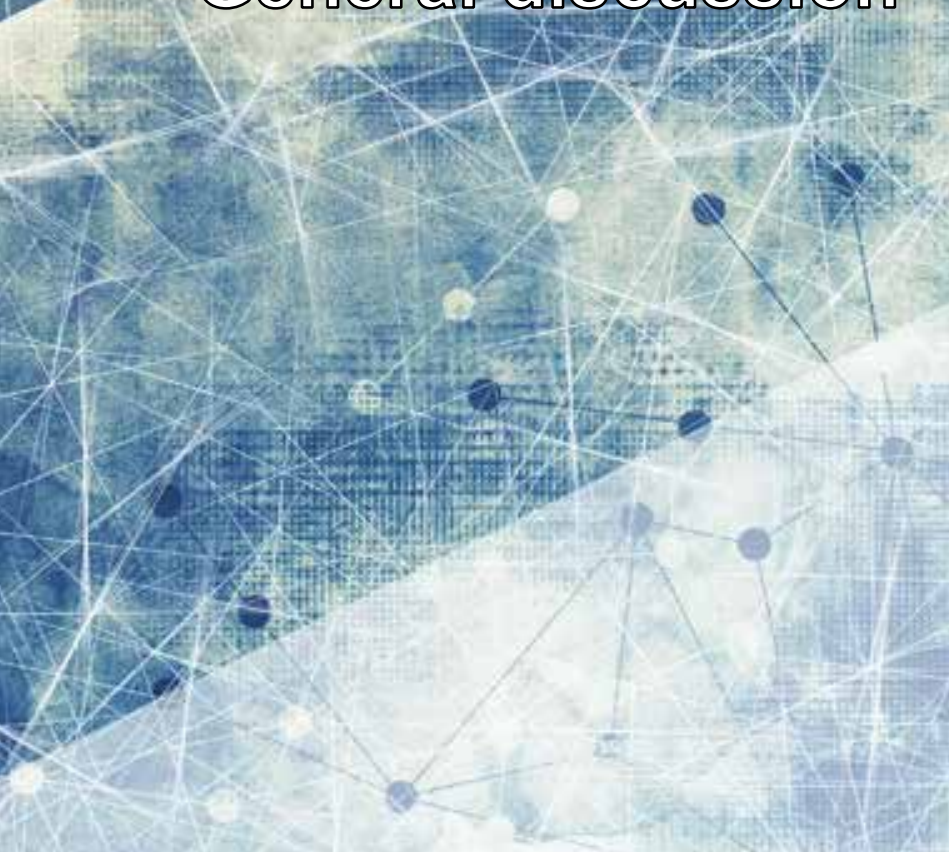

\section{teit}


Eye drops are currently the most prescribed drug delivery products for treatment and prevention of ocular diseases.[1] However, the use of eye drops has several drawbacks. Besides systemic side effects [2] and local toxicity due to preservatives $[3,4]$, the main disadvantages of eye drops are low drug bioavailability [5-7] and poor patient compliance.[8-10] Hence, there is need for new, innovative methods to mitigate these disadvantages.

The primary aim of this thesis was to provide a clear understanding of the mechanical, functional and clinical properties of the ocular coil. The ocular coil is a coiled and coated stainless steel wire filled with drug (ketorolac) releasing microspheres. The microspheres are kept in place by two dome-shaped caps, which also soften the extremities of the ocular coil. The ocular coil is placed in the inferior conjunctival fornix, where it releases its drug gradually to the tear film. Thereupon, the drug diffuses to the anterior chamber and anterior tissues.

Further on in this thesis, innovative techniques in ocular drug detection and objective scoring of ocular redness in relation to the ocular coil have been investigated. This chapter discusses the possible implications of the main results obtained in this Ph.D. research, critical considerations and future perspectives of ocular drug delivery.

\subsection{The ocular coil as drug delivery device}

In order to improve the way of delivering drugs to the eye, the Ocular Coil Drug Delivery Comfort (OCDC) project was launched. Within this project, the ocular coil (previously described as the OphthaCoil [11-15]) was redesigned and prepared for clinical testing. Earlier studies showed that the OphthaCoil that was filled with drugcoated metal filaments, was too rigid.[13] Hence, the filaments were changed into a more flexible drug eluting matrix of microspheres. The design and in vitro characteristics of the new ocular coil are described in detail in Chapter 3.

In Chapter 4 the pharmacokinetics of the drug-loaded ocular coil were evaluated in New Zealand White rabbits. The ocular coil showed high release of ketorolac during the first days that gradually lowered till day 28 . The efficacy of the ocular coil was evaluated after inducing a surgical ocular trauma in the rabbit's eye. The results show that the ocular coil is as effective as eye drops in suppressing an induced inflammation.

Remarkably, also in the untreated control group, inflammatory markers decreased quickly to baseline levels until day four after induction of the ocular trauma. This raises questions regarding the treatment duration that is actually needed. Currently, eye drops are prescribed for 28 days in order to suppress acute inflammation and to prevent cystoid macular edema (CME) after cataract surgery.[16] Further research is needed to demonstrate whether a shorter treatment, for example only during the first 48 hours postoperatively, is sufficiently effective.[17] 
In Chapter 5, a placebo-microsphere filled version of the ocular coil was evaluated in a single-center intervention study. Two designs of the ocular coil (a straight and a curved version) were tested in two cohorts of healthy human subjects for 28 days. Although both ocular coils were considered highly comfortable and satisfactory safe, retention time of the ocular coils was lower than expected. Dislocation and loss of the ocular coil was observed in the vast majority of subjects.

Design changes to enhance retention time, such as to increase the roughness or stickiness of the surface, to create overhanging caps holding the ocular coil in place during movements or to design a circular ocular coil, are very likely to affect the comfort of the ocular coil.

While 28 days of wear of the ocular coil is currently not achievable, the use of the ocular coil for short-term application, for example during the preoperative setting, might be better suited. In this case, the ocular coil could be filled with a NSAID (e.g., ketorolac) in combination with a mydriatic agent (e.g., phenylephrine) to reduce the acute inflammatory response while providing stable mydriasis during surgery. It has been shown that delivering ketorolac three days before surgery resulted in lower pain levels during and after the surgery.[18] However, postoperative treatment was still advised since drug concentrations at the end of the surgery were too low to be effective.[18,19]

The ocular coil can be loaded with any type of drug. The initial choice for ketorolac was based on its effective cyclooxygenase (COX) inhibition, generic availability and freedom to operate. Ketorolac is a non-selective COX-1 and COX-2 inhibitor, and is known to be the most potent COX-1 inhibitor, whereas amfenac (nepafenac is converted to amfenac), followed by bromfenac, are the most potent COX-2 inhibitors.[20] While monotherapy of ketorolac, nepafenac or bromfenac after cataract surgery is equally effective in the prevention of CME [21,22], a large prospective randomized control trial (the ESCRS PREMED study) headed by the University Eye Clinic Maastricht found that combination therapy of bromfenac and dexamethasone resulted in the lowest incidence of CME.[23] The potential of drug delivery devices such as the ocular coil to delivery multiple drugs simultaneously holds great promise.

Use of the ocular coil in veterinary medicine is also imaginable because administration of topical drugs to animals is often difficult and demanding. The retention issue may be less problematic in animals because in our animal study, due to the anatomical differences between a rabbit eye and a human eye, we fixated the ocular coil into the conjunctival fornix using a single stitch. This one-time stitching may still outweigh the burden of frequent administration of topical drugs to animals. 


\subsection{Raman spectroscopy: the relevance of non-invasive in vivo ocular drug detection}

Besides innovations in ocular drug delivery, also ocular drug detection is in need for an update of the technology. Currently, in vivo ocular drug detection requires invasive sampling and multiple sample processing steps. We investigated the use of non-invasive Raman spectroscopy for the detection of drugs in the anterior chamber. Raman spectroscopy is able to track a molecule in vivo in real-time. In Chapter 6 we describe the detection of ketorolac using Raman spectroscopy. While Raman spectroscopy performed comparable to high-performance liquid chromatography (HPLC) for in vitro samples, in vivo Raman spectroscopy was not sensitive enough to detect a clinically relevant dose of ketorolac in the anterior chamber. More research should be conducted to increase the sensitivity of Raman spectroscopy for non-invasive in vivo drug detection.

Raman signal processing proved to be challenging and complex. Currently we are unable to remove hardware-related as well as background noise from in vivo raw signals. Chapter 6 a describes the development of a MATLAB ${ }^{\circledR}$ tool to enhance the intensity of a Raman signal by lowering hardware-related artifacts and correcting for background fluorescence. This tool, together with the open-access nature of our dataset (Chapter 6b), will help us and other researchers towards the future realization of in vivo Raman spectroscopy.

Another future application of Raman spectroscopy within ophthalmology is for the detection of inflammatory markers in aqueous humor. Currently, floating cells and flare are used to indicate anterior chamber inflammation. So far, the detection of an anti-inflammatory response in cells by Raman spectroscopy has been demonstrated.[24] Raman spectroscopy has also been used to analyze an ex vivo corneal scrape of a patient and identified the presence of besifloxacin in the epithelial layers of the cornea.[25] Another Raman spectroscopy study showed biochemical changes in the tear fluid of a contact lens wearer, wearing two different types of contact lenses (hydrogel and si-hydrogel).[26] Besides ophthalmology, Raman spectroscopy has applications in other fields, e.g. for the detection of modified skin cells in the context of cancer diagnosis $[27,28]$, or for trans-dermal detection of blood glucose levels.[29]

\subsection{Deep-learning artificial intelligence to objectify ocular redness}

For the safety evaluation of the ocular coil, ocular redness (or hyperaemia) was one of the key parameters. Scoring of hyperaemia uses a five points image-based scale that was introduced in 1978 by Davies et al. [30] and updated in 2001 by Efron et 
al.[31] As this scoring method suffers from low sensitivity and high subjectivity, our aim was to develop a software to objectively quantify hyperaemia. Using deeplearning artificial intelligence $(\mathrm{Al})$, we developed an automatic segmentation tool to outline the sclera as region of interest (Chapter 7 ). It was noticed that unequal illumination of the eye (due to the light source of the slit-lamp and the round shape of the eye) complexified the eventual calculation of ocular redness. Further steps are needed to select the best illumination equipment and to standardize camera settings. Other future examples of deep-learning Al-based analysis of ocular images are the scoring of the severity of a hypopyon, a corneal ulcer, corneal neovascularization or corneal edema. The use of such a tool, that can objectively quantify an outcome, is highly preferred in clinical studies, particularly in large multi-center trials. Moreover, when provided as an application on a smartphone or tablet, it can be used during digital consulting of patients.

\subsection{References}

1. Urti, A., Challenges and obstacles of ocular pharmacokinetics and drug delivery. Adv Drug Deliv Rev, 2006. 58(11): p. 1131-1135.

2. Farkouh, A., Frigo, P., and Czejka, M., Systemic side effects of eye drops: a pharmacokinetic perspective. Clin Ophthalmol, 2016. 10: p. 2433-2441.

3. Kaszli, F.A. and Krieglstein, G.K., Tear film deficiencies pharmacology of eye drops and toxicity. Curr Opin Ophthalmol, 1996. 7(4): p. 12-16.

4. Palmer, R.M. and Kaufman, H.E., Tear film, pharmacology of eye drops, and toxicity. Curr Opin Ophthalmol, 1995. 6(4): p. 11-16.

5. Ramsay, E., Del Amo, E.M., Toropainen, E., Tengvall-Unadike, U., Ranta, V.P., Urtti, A., and Ruponen, M., Corneal and conjunctival drug permeability: Systematic comparison and pharmacokinetic impact in the eye. Eur J Pharm Sci, 2018. 119: p. 83-89.

6. Del Amo, E.M., Rimpela, A.K., Heikkinen, E., Kari, O.K., Ramsay, E., Lajunen, T., Schmitt, M., Pelkonen, L., Bhattacharya, M., Richardson, D., Subrizi, A., Turunen, T., Reinisalo, M., Itkonen, J., Toropainen, E., Casteleijn, M., Kidron, H., Antopolsky, M., Vellonen, K.S., Ruponen, M., and Urtti, A., Pharmacokinetic aspects of retinal drug delivery. Prog Retin Eye Res, 2017. 57: p. 134-185.

7. Pelkonen, L., Tengvall-Unadike, U., Ruponen, M., Kidron, H., Del Amo, E.M., Reinisalo, M., and Urtti, A., Melanin binding study of clinical drugs with cassette dosing and rapid equilibrium dialysis inserts. Eur J Pharm Sci, 2017. 109: p. 162-168.

8. Olthoff, C.M., Hoevenaars, J.G., van den Borne, B.W., Webers, C.A., and Schouten, J.S., Prevalence and determinants of non-adherence to topical hypotensive treatment in Dutch glaucoma patients. Graefes Arch Clin Exp Ophthalmol, 2009. 247(2): p. 235-243.

9. An, J.A., Kasner, O., Samek, D.A., and Levesque, V., Evaluation of eyedrop administration by inexperienced patients after cataract surgery. J Cataract Refract Surg, 2014. 40(11): p. 18571861.

10. Bertens, C.J.F., Gijs, M., van den Biggelaar, F., and Nuijts, R., Topical drug delivery devices: A review. Exp Eye Res, 2018. 168: p. 149-160.

11. Pijls, R.T., Sonderkamp, T., Daube, G.W., Krebber, R., Hanssen, H.H., Nuijts, R.M., and Koole, L.H., Studies on a new device for drug delivery to the eye. Eur J Pharm Biopharm, 2005. 59(2): p. 283-288.

12. Pijls, R.T., Lindemann, S., Nuijts, R.M.M.A., Daube, G.W., and Koole, L.H., Pradofloxacin release from the OphthaCoil: a new device for sustained delivery of drugs to the eye. Journal of Drug Delivery Science and Technology, 2007. 17(1): p. 87-91.

13. Pijls, R.T., Hanssen, H.H., Nuijts, R.M., and Koole, L.H., Flexible coils with a drug-releasing hydrophilic coating: a new platform for controlled delivery of drugs to the eye? Biomed Mater Eng, 2004. 14(4): p. 383-393. 
14. Pijls, R.T., Hanssen, H.H., Nuijts, R.M., Daube, G.W., and Koole, L.H., In vivo tolerance and kinetics of a novel ocular drug delivery device. J Control Release, 2006. 116(2): p. e47-49.

15. Pijls, R.T., Cruysberg, L.P., Nuijts, R.M., Dias, A.A., and Koole, L.H., Capacity and tolerance of a new device for ocular drug delivery. Int J Pharm, 2007. 341(1-2): p. 152-161.

16. Yilmaz, T., Cordero-Coma, M., and Gallagher, M.J., Ketorolac therapy for the prevention of acute pseudophakic cystoid macular edema: a systematic review. Eye (Lond), 2012. 26(2): p. 252-258.

17. Waterbury, L.D., Galindo, D., Villanueva, L., Nguyen, C., Patel, M., Borbridge, L., Attar, M., Schiffman, R.M., and Hollander, D.A., Ocular penetration and anti-inflammatory activity of ketorolac $0.45 \%$ and bromfenac $0.09 \%$ against lipopolysaccharide-induced inflammation. $\mathrm{J}$ Ocul Pharmacol Ther, 2011. 27(2): p. 173-178.

18. Donnenfeld, E.D., Perry, H.D., Wittpenn, J.R., Solomon, R., Nattis, A., and Chou, T., Preoperative ketorolac tromethamine $0.4 \%$ in phacoemulsification outcomes: pharmacokineticresponse curve. J Cataract Refract Surg, 2006. 32(9): p. 1474-1482.

19. Katsev, D.A., Katsev, C.C., Pinnow, J., and Lockhart, C.M., Intracameral ketorolac concentration at the beginning and end of cataract surgery following preoperative topical ketorolac administration. Clin Ophthalmol, 2017. 11: p. 1897-1901.

20. Walters, T., Raizman, M., Ernest, P., Gayton, J., and Lehmann, R., In vivo pharmacokinetics and in vitro pharmacodynamics of nepafenac, amfenac, ketorolac, and bromfenac. J Cataract Refract Surg, 2007. 33(9): p. 1539-1545.

21. Jung, J.W., Chung, B.H., Kim, E.K., Seo, K.Y., and Kim, T.I., The Effects of Two Non-Steroidal Anti-Inflammatory Drugs, Bromfenac $0.1 \%$ and Ketorolac 0.45\%, on Cataract Surgery. Yonsei Med J, 2015. 56(6): p. 1671-1677.

22. Almeida, D.R., Khan, Z., Xing, L., Bakar, S.N., Rahim, K., Urton, T., and El-Defrawy, S.R., Prophylactic nepafenac and ketorolac versus placebo in preventing postoperative macular edema after uneventful phacoemulsification. J Cataract Refract Surg, 2012. 38(9): p. 15371543.

23. Wielders, L.H.P., Schouten, J., Winkens, B., van den Biggelaar, F., Veldhuizen, C.A., Findl, O., Murta, J.C.N., Goslings, W.R.O., Tassignon, M.J., Joosse, M.V., Henry, Y.P., Rulo, A.H.F., Guell, J.L., Amon, M., Kohnen, T., Nuijts, R., and Group, E.P.S., European multicenter trial of the prevention of cystoid macular edema after cataract surgery in nondiabetics: ESCRS PREMED study report 1. J Cataract Refract Surg, 2018. 44(4): p. 429-439.

24. da Silva, E.C.O., dos Santos, F.M., Ribeiro, A.R.B., de Souza, S.T., Barreto, E., and Fonseca, E.J.d.S., Drug-induced anti-inflammatory response in A549 cells, as detected by Raman spectroscopy: a comparative analysis of the actions of dexamethasone and p-coumaric acid. Analyst, 2019. 144(5): p. 1622-1631.

25. Palileo, C. and McDermott, M., Confirmation of Besifloxacin Corneal Precipitation After Cataract Surgery by Raman Spectroscopy. Ophthalmology, 2019. 126(11): p. 1548.

26. Capaccio, A., Sasso, A., and Rusciano, G., Raman Analysis of Tear Fluid Alteration Following Contact Lense Use. Sensors (Basel), 2019. 19(15).

27. Zhao, J., Zeng, H., Kalia, S., and Lui, H., Using Raman Spectroscopy to Detect and Diagnose Skin Cancer In Vivo. Dermatol Clin, 2017. 35(4): p. 495-504.

28. Zhang, X., Yu, F., Li, J., Song, D., Li, H., Wang, K., He, Q., and Wang, S., Investigation on the Cancer Invasion and Metastasis of Skin Squamous Cell Carcinoma by Raman Spectroscopy. Molecules, 2019. 24(11).

29. Shih, W.C., Bechtel, K.L., and Rebec, M.V., Noninvasive glucose sensing by transcutaneous Raman spectroscopy. J Biomed Opt, 2015. 20(5): p. 051036.

30. Ruben, M., Soft Contact Lenses: Clinical and Applied Technology. 1978: John Wiley \& Sons.

31. Efron, N., Morgan, P.B., and Katsara, S.S., Validation of grading scales for contact lens complications. Ophthalmic Physiol Opt, 2001. 21(1): p. 17-29. 
General discussion 


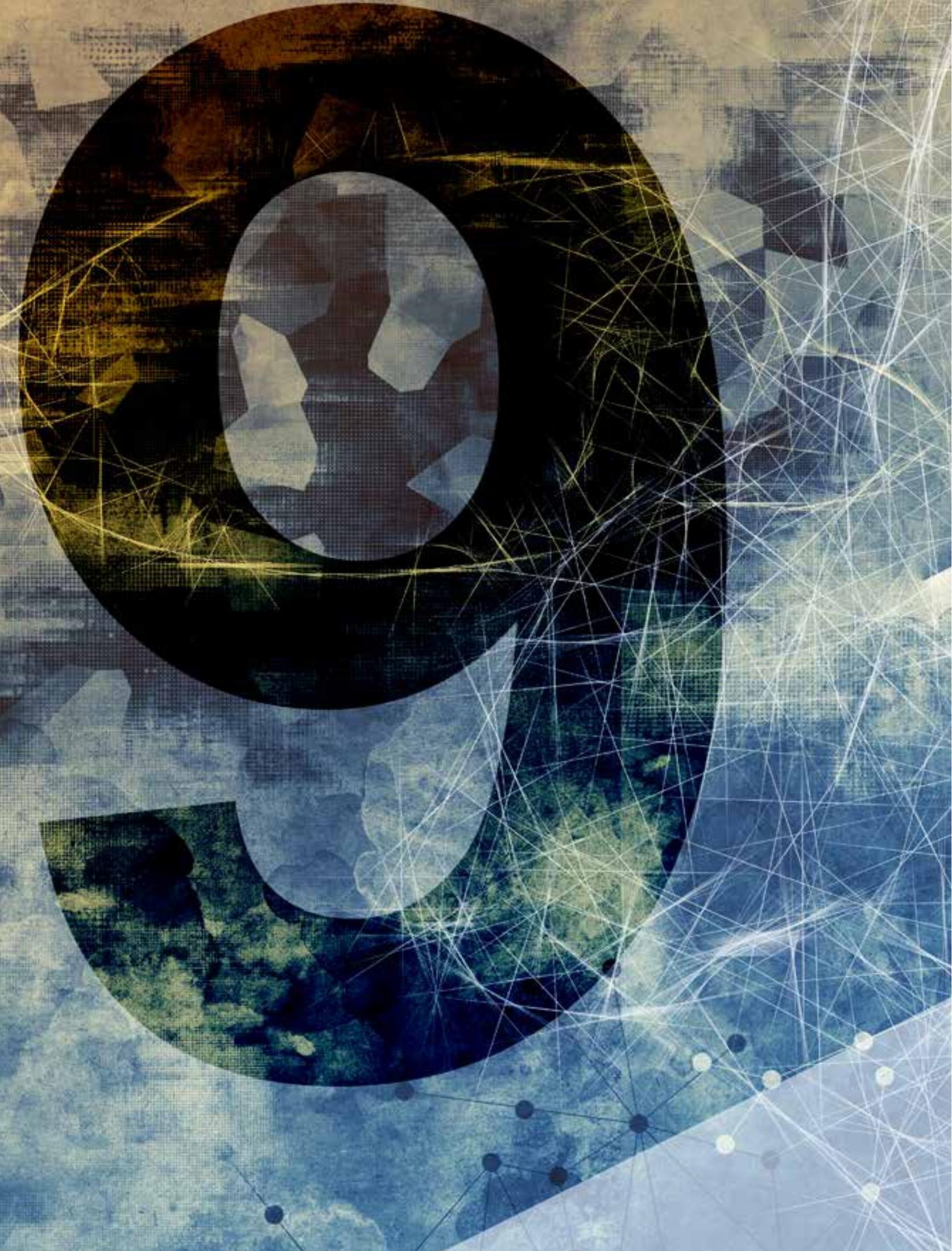




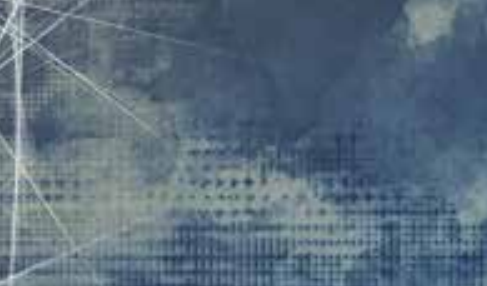

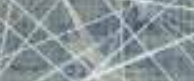

Pe.

Chapter 9.

\section{Impaet}

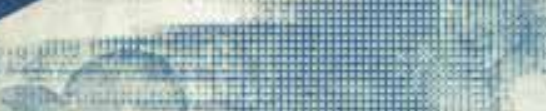


Aging of the population leads to an increase in age-related visual impairment and blindness.[1] According to the report by the World Health Organization (WHO), 2.2 billion people are suffering from vision impairment globally. One billion of the visually impaired patients could have been prevented or has yet to be addressed.[2] This includes moderate or severe distance vision impairment or blindness due to unaddressed refractive error (123.7 million), cataract (65.2 million), glaucoma (6.9 million), corneal opacities (4.2 million), diabetic retinopathy (3 million), and trachoma ( 2 million), as well as near vision impairment caused by unaddressed presbyopia (826 million).[1]

\subsection{Clinical relevance of the ocular coil}

Current patterns of topical ophthalmic drug delivery fail because low drug absorption due to short residence time on the ocular surface and high pre-corneal drug loss. This requires the need for frequent drug administration, which then again is causing low patient compliance.[3] To improve drug delivery, other routes than eye drops are used (Chapter 2). Implants or direct injections (e.g. subconjunctival, subtenon, intracameral, intravitreal) into the targeted site can be used but are invasive and only achieve suboptimal drug levels. For example, intravitreal injections of anti-VEGF for age-related macular degeneration need to be repeated regularly and have poor patient tolerance (pain and fear), significant risks (e.g. endophthalmitis), and increased costs (loss of working hours) and manpower requirements.

The ocular coil is a non-invasive drug delivery device. Ideally, it is put in place once by the physician and remains there for a prolonged period of time. As such, it would replace the need for self-administration of eye drops. This would be beneficial for e.g. aged persons that have difficulty to self-apply eye drops or patients with corneal ulcers who have to apply medication as often as once an hour. In both cases, eye drop administration is currently performed by a healthcare worker and comes with healthcare costs. The associated healthcare costs are significant.

Patient non-compliance with eye drops is prevalent, both for short-term use (e.g. after cataract surgery) as well as for chronic disease requiring lifelong use of eye drops (e.g. glaucoma). Reasons for non-compliance are forgetfulness, incorrect instilment, fear, and physical or cognitive limitations of aged persons. Consequences of poor compliance after cataract surgery are e.g. endophthalmitis (antibiotic eye drops) and cystoid macular edema (CME) (anti-inflammatory eye drops). Both complications can permanently impact vision. By bypassing patient compliance, the ocular coil may be able to reduce the incidence of those complications. In the end, this strategy may also be less expensive than the treatment of those complications. 


\subsection{The ocular drug delivery device market}

In the treatment and prevention of ocular diseases, eye drops and ointments are often the first line of defense. Therefore, ophthalmic drug delivery represents a significant economic value. The global ophthalmic drugs market size was valued at $\$ 30.3$ billion in 2018 and is expected to grow to $\$ 43.1$ billion 2026 with a compound annual growth rate of $4.5 \%$.[4] The US was the largest market for ophthalmic drugs, accounting for $40 \%$ of the global market. Five major EU countries (UK, DE, IT, FR, and ES) formed $18 \%$ of the global ophthalmic market.[5] Implementation of a new successful drug delivery method may have a significant impact on the field. Nevertheless, it is challenging for new drug delivery devices to enter the market. Tight rules and regulations of the Food and Drug Administration (FDA) and European Union's Public Health Division and European Medicine Agency (EMA) make it time consuming and expensive to bring a drug delivery device to the market. Hence, drug delivery devices can hardly compete with the low costs of eye drops. The advantage of drug delivery devices should considerably outweigh the total costs of the devices. One successful example is the ocular drug insert for preoperative pupil dilation (Mydriasert, TheaPharma), that is being used by $30 \%$ of Dutch cataract surgeons.[6] Benefits of this insert include the significantly decreased nurse time, number of gestures, and equal pupil dilation as compared to eye drops.[7] A recent costeffectiveness study of different mydriatics before cataract surgery showed that intracameral injection is more expensive $(€ 17)$ than eye drops $(€ 5)$ or a mydriatic insert $(€ 7)$ and results in the smallest pupil dilation. Due to the price, implementation could be considered when there is limited availability of nurses or physical space for perioperative care.[8]

Another drug delivery device that made it to the market is Dextenza ${ }^{\mathrm{TM}}$ (Ocular Therapeutix Inc. (Bedford, MA, US), a $0.4 \mathrm{mg}$ dexamethasone loaded punctum plug that was FDA approved in November 2018.[9] Dextenza ${ }^{\mathrm{TM}}$ is injected at the end of cataract surgery and is the first punctum insert that can provide sustained release up to 30 days. Postsurgical treatment with Dextenza ${ }^{\mathrm{TM}}$ costs about $€ 409$ (\$450) and it takes about $25 \%$ of the postsurgical market since its release. A similar device is Dexycu $^{\circledR}$ (EyePoint Pharmaceuticals, Watertown, MA, US).[10] Dexycu ${ }^{\circledR}$ is a 517 $\mu \mathrm{g}$ intracameral dexamethasone insert to be injected at the end of cataract surgery. The price of Dexycu ${ }^{\circledR}$ treatment is about $€ 541$ (\$595) and has proven to be more effective than 30 days eye drop therapy.[11]

Another potential alternative to postoperative eye drops is Omidria ${ }^{\circledR}$ (Omeros pharmaceutics). Omidria ${ }^{\circledR}$ is a drug combination of ketorolac and phenylephrine included in the irrigation fluid that is used during surgery. Thus far, results show less pain during and after the surgery $[12,13]$ and a lower incidence in post-operative CME.[13] The price for one bottle of Omidria $^{\circledR}$ is about $€ 470$ ( $\left.£ 400\right)$ [personal 
communication]. However, the use of drugs for preoperative mydriasis is still needed and postoperative anti-inflammatory treatment is also advised.

\subsection{Raman spectroscopy for (pre)clinical use}

Tracking drugs through ocular tissue is a must in the field of ocular pharmacokinetics. It offers information on the achievable intraocular drug concentrations of a new drug delivery device. Raman spectroscopy is a non-invasive technique that is able to detect drugs in vivo. It is able to penetrate through ocular tissue and to provide detailed information on molecules and structures inside the eye.

Raman spectroscopy can be of significant value for drug related studies. Current methods include harvesting ocular tissues or fluids during surgery, but only provides information at a single point in time. Kinetic or real-time information can only be obtained from large cohorts of laboratory animals (e.g. rabbits, dogs, pigs, and monkeys).[14] This technique could therefore lower the number of animals used for pharmacokinetic experiments.

One example of clinical use of Raman spectroscopy could be used to identify the causative micro-organism in a patient with endophthalmitis. Currently, it takes a few days before this information is available (due to bacterial culture time) and the start of the correct treatment (e.g. specific antibiotic or antiviral) is delayed. Immediate identification on-site may increase the success-rate of the treatment. Other potential examples of clinical use of Raman spectroscopy include detection of inflammatory cytokine for corneal dystrophies [15] or insulin [16] for diabetes.

\subsection{Quantification of ocular redness}

The degree of ocular redness serves as an important diagnostic feature for the diagnosis and monitoring of ocular diseases. Furthermore, it is an indicator of the safety level of a new ocular drug. Objective scoring of ocular redness remains however difficult. In this context, a tool to quantify ocular redness can be of use in both clinical and research settings. In the future, it can be further optimized to determine other features, such as e.g. the severity of a hypopion, limbal redness and corneal neovascularization.

A (pilot) portable version of the tool has been developed for smartphones. This offers clinicians and researchers the possibility to monitor ocular redness of a specific patient over time. For example, after surgery it could be used to track recovery of the ocular tissue and the surgeon could adapt accordingly its treatment strategy to it. This tool may bring ophthalmology closer to personalized medicine. 


\subsection{References}

1.

Bourne, R.R.A., Flaxman, S.R., Braithwaite, T., Cicinelli, M.V., Das, A., Jonas, J.B., Keeffe, J., Kempen, J.H., Leasher, J., Limburg, H., Naidoo, K., Pesudovs, K., Resnikoff, S., Silvester, A., Stevens, G.A., Tahhan, N., Wong, T.Y., Taylor, H.R., and Vision Loss Expert, G., Magnitude, temporal trends, and projections of the global prevalence of blindness and distance and near vision impairment: a systematic review and meta-analysis. Lancet Glob Health, 2017. 5(9): p. e888-e897.

2. WHO. Blindness and vision impairment, https://www.who.int/news-room/factsheets/detail/blindness-and-visual-impairment, (accessed 28 Nov 2019), online.

3. Bertens, C.J.F., Gijs, M., van den Biggelaar, F., and Nuijts, R., Topical drug delivery devices: A review. Exp Eye Res, 2018. 168: p. 149-160.

4. Grand View Research. Ophthalmic Drugs Market Worth \$43.1 Billion By 2026 | CAGR 4.5\%, https://www.grandviewresearch.com/press-release/global-ophthalmic-therapeutics-drugmarket, (accessed 28 Nov 2019), online.

5. Mordor Intelligence. EUROPE OPHTHALMOLOGY DRUG AND DEVICE MARKET GROWTH, TRENDS, AND FORECAST (2019 - 2024), https://mordorintelligence.com/industryreports/european-opthalmic-drugs-devices-market-industry, (accessed 28 Nov 2019), online.

6. Behndig, A., Cochener, B., Guell, J.L., Kodjikian, L., Mencucci, R., Nuijts, R.M., Pleyer, U., Rosen, P., Szaflik, J.P., and Tassignon, M.J., Endophthalmitis prophylaxis in cataract surgery: overview of current practice patterns in 9 European countries. J Cataract Refract Surg, 2013. 39(9): p. 1421-1431.

7. Caruba, T., Couffon-Partant, C., Oliary, J., Tadayoni, R., Limelette, N., and Gaudric, A., [Efficacy and efficiency of preoperative mydriasis: drops versus ocular insert]. J Fr Ophtalmol, 2006. 29(7): p. 789-795.

8. Simons, R.W.P., Rondas, L.U.E., van den Biggelaar, F., Berendschot, T., Visser, N., de Crom, R., and Nuijts, R., Cost analysis of mydriasis strategies in cataract surgery care in the Netherlands. J Cataract Refract Surg, 2021.

9. Drugs.com. Dextenza Approval History, https://www.drugs.com/history/dextenza.html, (accessed 30 Mar 2020) Oline.

10. Nasdaq. Ocular Therapeutix: A Diamond In The Rough As Dextenza Is Finally Launching With The Right Attributes For Success, https://www.nasdaq.com/articles/ocular-therapeutix\%3A-adiamond-in-the-rough-as-dextenza-is-finally-launching-with-the-right, (accessed $30 \mathrm{Mar} 2020$ ), online.

11. Donnenfeld, E. and Holland, E., Dexamethasone Intracameral Drug-Delivery Suspension for Inflammation Associated with Cataract Surgery: A Randomized, Placebo-Controlled, Phase III Trial. Ophthalmology, 2018. 125(6): p. 799-806.

12. Walter, K., Delwadia, N., and Coben, J., Continuous intracameral phenylephrine-ketorolac irrigation for miosis prevention in femtosecond laser-assisted cataract surgery: Reduction in surgical time and iris manipulation. J Cataract Refract Surg, 2019. 45(4): p. 465-469.

13. Visco, D.M. and Bedi, R., Effect of intracameral phenylephrine $1.0 \%$-ketorolac $0.3 \%$ on postoperative cystoid macular edema, iritis, pain, and photophobia after cataract surgery. J Cataract Refract Surg, 2020. 46(6): p. 867-872.

14. Schoenwald, R.D., Ocular drug delivery. Pharmacokinetic considerations. Clin Pharmacokinet, 1990. 18(4): p. 255-269.

15. Shetty, R., Naidu, J.R., Nair, A.P., Vaidya, T.A., D'Souza, S., Matalia, H., Deshpande, V., Sethu, S., Ghosh, A., and Chakrabarty, K., Distinct ocular surface soluble factor profile in human corneal dystrophies. Ocul Surf, 2020. 18(2): p. 237-248.

16. Rocha, E.M., Cunha, D.A., Carneiro, E.M., Boschero, A.C., Saad, M.r.J.A., and Velloso, L.c.A., Identification of Insulin in the Tear Film and Insulin Receptor and IGF-I Receptor on the Human Ocular Surface. Investigative Ophthalmology \& Visual Science, 2002. 43(4): p. 963-967. 


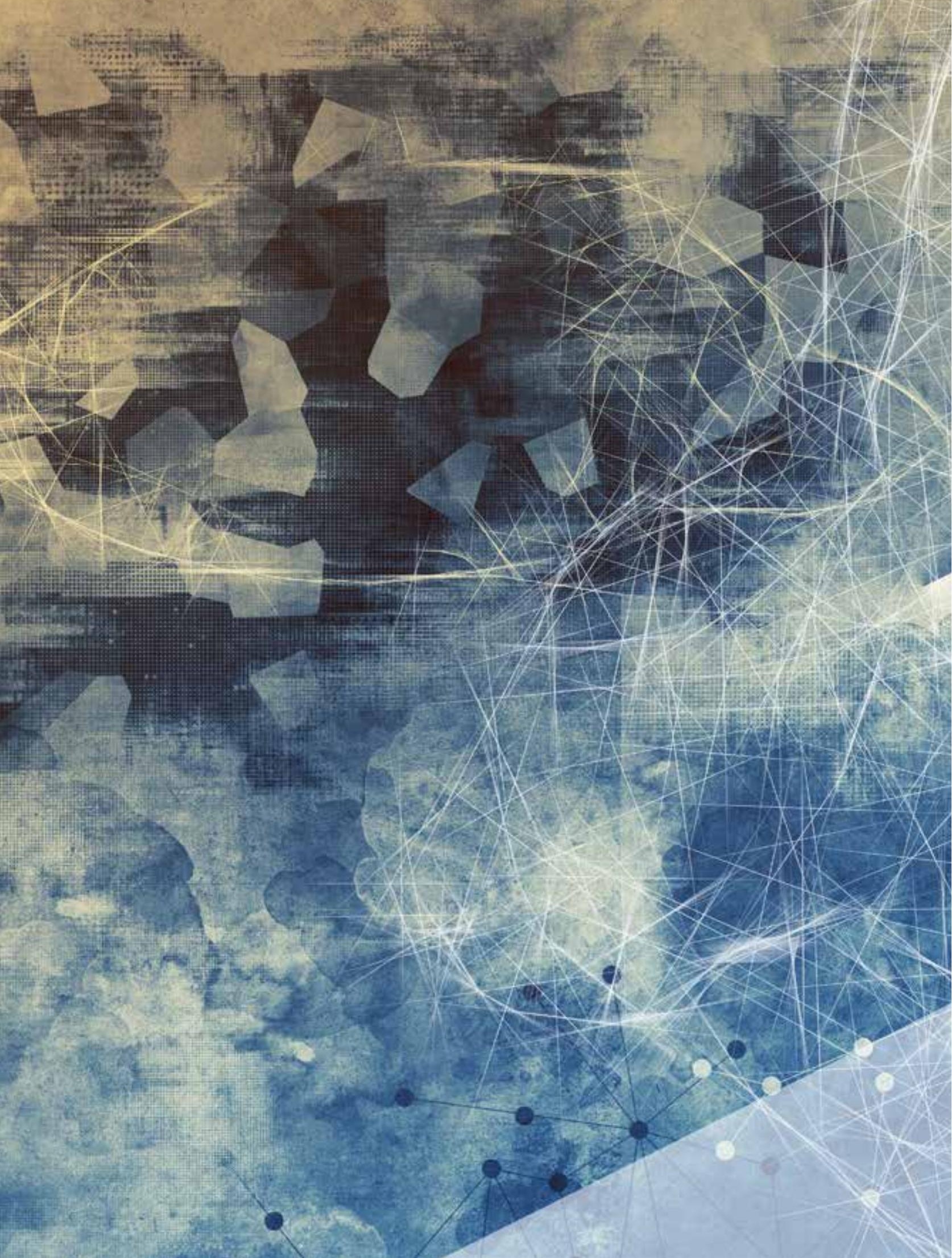


For treatment and prevention of ocular diseases, ocular drugs are most commonly applied as eye drops. Although eye drops are widely used, the use of eye drops can result in local (e.g. toxicity) or systemic (e.g. allergy) side effects. However, the main disadvantages of eye drops are its low drug bioavailability and the limited number of patients that adhere to the prescribed regime (called patient compliance). In general, less than $5 \%$ of the applied drug will reach the target side. Therefore, eye drops need to be applied multiple times a day, but this lowers its patient compliance. Other reasons for low patient compliance are the fact that patients forget to instill the eye drops $(27 \%)$, they do not have eye drops available at the moment of instillment $(20 \%)$, or they are not able to instill the eye drop themselves $(16 \%)$.

To overcome the disadvantages of eye drops, drug delivery devices have been designed. An overview of the state of the art drug delivery devices for ophthalmic uses has been provided in Chapter 2. These devices can deliver drugs to the eye via two different routes, the corneal drug delivery route or the scleral drug delivery route. The devices in this chapter are organized by their shape. There are oval- and ring- shaped devices (Ocusert ${ }^{\circledR}$ and Helios ${ }^{T M}$ ), rod-shaped devices (Mydriasert ${ }^{\circledR}$, Ocufit $\mathrm{SR}^{\circledR}$ and the OphthaCoil), punctum plugs (Evolute ${ }^{\circledR}$ and Dextenza ${ }^{\circledR}$ ), and contact lenses and corneal shields. A complete overview is provided in table 2-2 and figure 2-2. Many of these devices have proven to deliver drugs more efficiently than to eye drops.

The aim of this thesis was to investigate an innovative method for ocular drug delivery. This was embedded within the Chemelot Institute for Science and Technology (InSciTe) Ocular Coil Drug delivery and Comfort (OCDC) project. Within this project drug delivery via an ocular coil was tested. The ocular coil is a coiled and coated stainless steel wire filled with microspheres. The microspheres are kept in place by two dome-shaped caps, which also soften the extremities of the ocular coil. The ocular coil is placed in the inferior conjunctival fornix, where it releases its drug gradually to the tear film. Chapter 3 provides an overview of the design and the drug release mechanism of this device. Moreover, the effect of different thicknesses in combination with the coil diameter was tested to determine the optimal flexibility and coil filling capacity. Furthermore, a filling method was developed and escape of microspheres from the ocular coil was tested in different conditions. When the ocular coil was filled with microspheres with a diameter of $150 \pm 10 \mu \mathrm{m}$, no escape was observed when overstretching the ocular coil up till twice its length. To determine the curvature of the ocular coil in the inferior conjunctival fornix, a CT-scan was made. In parallel, drug (ketorolac) encapsulated poly-methyl methacrylate (PMMA) microspheres were developed. Ketorolac is a non-steroidal anti-inflammatory drug (NSAID), which is often prescribed after cataract surgery. Furthermore, PMMA is a 
polymer that is frequently used in ophthalmic products e.g. intraocular lenses and contact lenses. In vitro drug release studies showed high release of ketorolac $(50 \%$ of the original loading) in the first three days followed by sustained release of ketorolac up to 28 days (till $70 \%$ of the loaded drug).

The efficacy of the ocular coil was tested in New Zealand White rabbits (see Chapter 4). To investigate the pharmacokinetics of the ocular coil, the concentration ketorolac was determined in tears, aqueous humor and in plasma. In tears, the concentration ketorolac was higher at 4 and 24 hours in the ocular coil group, compared to the eye drop group. On days 4, 7 and 28 , the concentration ketorolac in the ocular coil group was equal to the concentration of the eye drop group. In aqueous humor the ketorolac concentration was only higher after 4 hours in the ocular coil group. On days 4,7 , and 28 the ketorolac concentration in the eye drop group exceeded that of the ocular coil group. This trend was also observed with the ketorolac concentration in plasma.

Furthermore, the potential to inhibit inflammation by the ocular coil was investigated. At first, a large sample (150-175 $\mu \mathrm{L})$ aqueous humor was drawn from the anterior chamber, to mimic a surgically induced anterior chamber trauma. Hereafter, either the ketorolac loaded ocular coil was inserted in the inferior conjunctival fornix, or ketorolac containing eye drops were applied three times a day, or no treatment was offered (control group). The total protein concentration was determined, as was the concentration of inflammatory markers such as prostaglandin $E_{2}$ (PGE2), tumor necrosis factor- $\alpha$ (TNF- $\alpha$ ), interleukin (IL)- 6 and IL-1 $\beta$, in tears, in aqueous humor and in plasma. Elevated $\mathrm{PGE}_{2}$ and IL-6 concentrations in aqueous humor in the control group confirmed that 4, 8 and 24 hours after inducing trauma inflammation was provoked. Treatment with the ocular coil or eye drops showed to hamper this inflammatory cascade. The ocular coil provided a stronger inhibition of $\mathrm{PGE}_{2}$ and IL6 compared to eye drops. Results of this study indicate that sustained release of drugs is as effective as interval-treatment with eye drops.

In a clinical study, the safety and comfort of the ocular coil (placebo version) was evaluated (see Chapter 5). Two different shapes of ocular coil were tested, a straight version and a curved version. In total, 21 subjects wore the straight ocular coil and 21 subjects wore the curved ocular coil. The retention time indicated the number of days that a subject could wear the ocular coil before it was removed or prematurely lost. The median retention time of the straight ocular coil was 5 days and 12 days for the curved ocular coils. In the end, only four subjects achieved to wear the straight ocular coil for the intended duration (28 days), and six subjects the curved ocular coil. Loss of the ocular coil was mostly observed when the subjects rubbed their eyes or while sleeping. Safety of the ocular coil was monitored using the Efron's grading 
scale. This scale scores conjunctival hyperaemia, limbal hyperaemia and corneal neovascularization. While wearing the ocular coil, only minor differences in conjunctival and limbal hyperaemia were observed. Moreover, no increase in corneal neovascularization was observed. A major drawback of the ocular coil was dislocation towards the nasal side of the conjunctival fornix. The curved ocular coil even migrated to the superior conjunctival fornix in three subjects.

To determine comfort of the ocular coil, a questionnaire was presented to the subjects. The questionnaire contained questions such as "I feel the presence of the ocular coil in my eye" and "presence of the ocular coil in my eye is uncomfortable". Most subjects was not aware of the presence of the ocular coil in the conjunctival fornix, nor was presence uncomfortable. Furthermore, we asked the subjects to score comfort of the ocular coil from 0 (very painful) to 100 (excellent comfort). An average comfort score of 88 was obtained for the straight and 93 for the curved ocular coil. Hence, we concluded that the ocular coil is safe and comfortable but the current retention time is too low to guarantee 28 days of sustained drug release.

A second topic of this thesis is use of Raman spectroscopy for the determination of drug concentrations in the eye. Raman spectroscopy is able to identify molecules based on energy changes in reflective light. This makes it possible to gain insight into molecules within a tissue without damaging it. In Chapter 6, the ketorolac concentration in the eye was quantified using Raman spectroscopy. First, the ketorolac concentration was investigated in post-mortem porcine eyes that were soaked in different concentrations ketorolac solution. Using three different focus lenses (f60 lens with a one-mirror gonioscopic lens, $25 x$ microscope objective (Jena lens) and a f80 lens) the most optimum conditions were selected for in vivo drug detection (full methods and calibration of the system is discussed in Chapter 6a). Furthermore, a MATLAB tool was developed to optimize the Raman signal and to remove background noise. While the Jena lens was able to detect drugs in a cuvette, this lens was unable to quantify drugs in vivo. Thereafter, New Zealand White rabbits received ketorolac containing eye drops three times a day. To assure no laser damage was done to the eye, the drug concentration were measured using the gonioscopic lens. However, Raman spectroscopy was not sensitive enough to quantify intraocular drug concentrations. The complete dataset is published in Chapter $\mathbf{6 b}$. With this data, other scientists can optimize their Raman spectroscope set-up and results. Furthermore, it can also be used to develop and validate new data analysis software.

The final part of this thesis focuses on the objectification of ocular hyperaemia by using a deep-learning software tool (see Chapter 7). Ocular hyperaemia is currently graded based on reference figures or drawings e.g. Efron's grading scale. Although 
this method is easy and fast, large variations between observers have been observed. Furthermore, a grading scale often has 4 or 5 scores which are not able to detect minor changes. Hence, automated quantification of ocular hyperaemia was investigated. First, the algorithm was trained by using machine learning to select the correct area of the sclera (segmentation). The results indicated that the automated program was better in segmenting the sclera than manually. The second part calculated ocular hyperaemia based on previously published algorithms. The algorithm of Amparo et al. and Sárándi et al. were most suitable for this.

All results from this thesis are discussed in more detail in Chapter 8. 


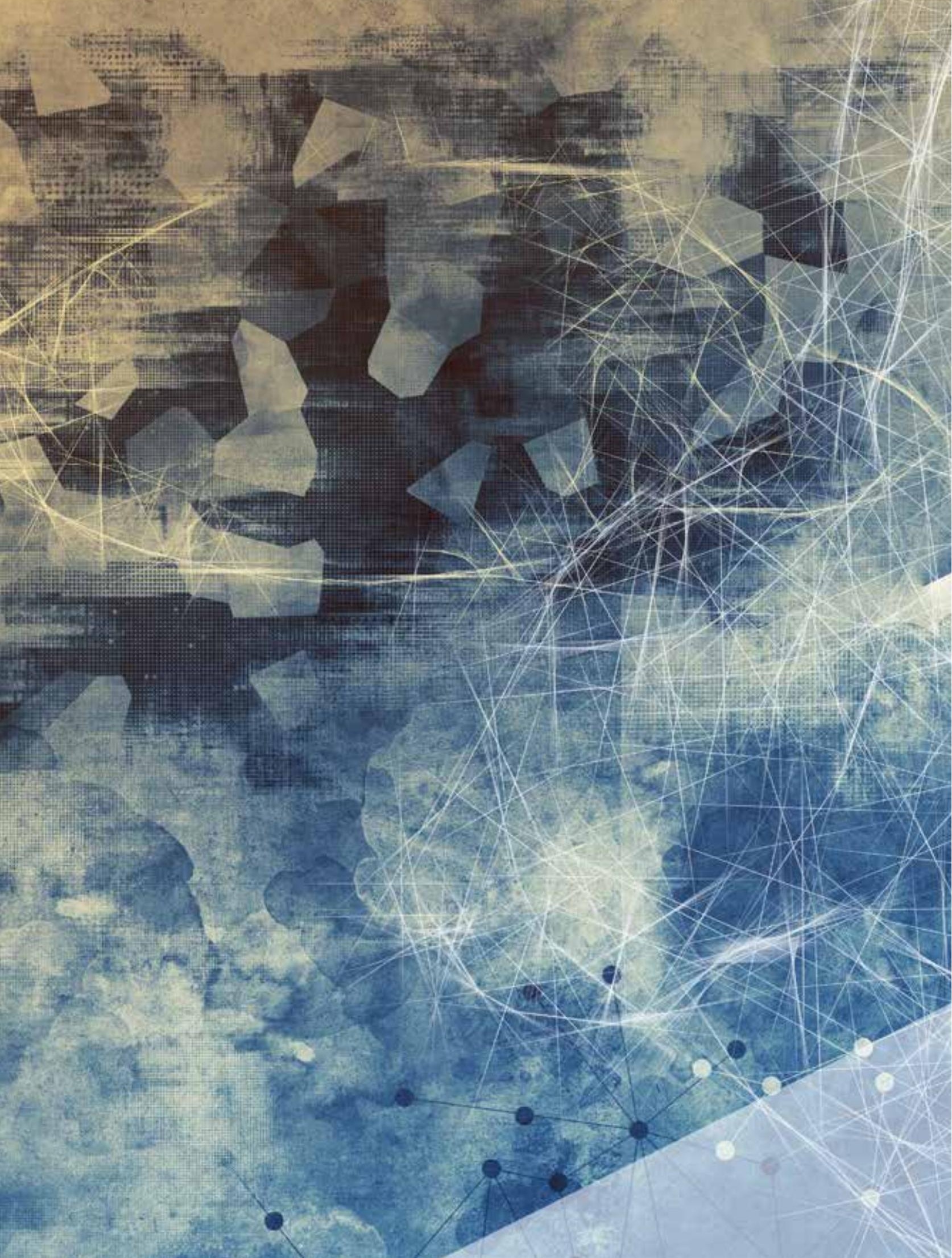


Oogdruppels zijn de meest voorgeschreven vorm van medicatie voor oogheelkundige aandoeningen. Desalniettemin kan het gebruik van oogdruppels resulteren in zowel systemische (b.v. allergie) als lokale (b.v. toxiciteit) bijwerkingen. Echter de grootste nadelen van oogdruppels zijn de lage opname van het medicijn door het oog en het geringe aantal patiënten dat therapietrouw is. Minder dan $5 \%$ van de daadwerkelijk toegediende medicijndosis komt in het oog terecht. Dit komt onder andere doordat tranen het medicijn snel wegspoelen. Daarom dienen oogdruppels vaak meerdere malen per dag toegediend te worden. Het resultaat hiervan is dat de therapietrouw van patiënten vermindert. Dit komt voornamelijk door het vergeten van het druppelmoment (27\%), de oogdruppels niet bij de hand hebben op het druppelmoment (20\%) en niet in staat zijn om zelf de oogdruppel toe te dienen $(16 \%)$.

Om deze nadelen van oogdruppels tegemoet te komen, kunnen medicijnafgifte apparaten (drug delivery devices) gebruikt worden. Een overzicht van een aantal medicijnafgifte apparaten voor het oog is beschreven in hoofdstuk 2. Deze apparaten kunnen het medicijn aan het oog geven via twee routes $\mathrm{nl}$. via het hoornvlies (de corneale route) of via de witte oogrok (de sclerale route). De apparaten hebben we gegroepeerd naar vorm. Zo zijn er ovaal- en ringvormige apparaatjes (de Ocusert ${ }^{\circledR}$ en de Helios $^{\mathrm{TM}}$ ) en staafvormige apparaten (de Mydriasert ${ }^{\circledR}$, Ocufit $\mathrm{SR}^{\circledR}$ en de voorloper van de oculaire coil: de OphthaCoil). Tevens is er gekeken naar punctumpluggen (Evolute ${ }^{\circledR}$ en Dextenza ${ }^{\circledR}$ ), contactlenzen en een kleine groep met andere apparaten. Een volledig overzicht kan gevonden worden in tabel 2-2 en figuur 2-2. Vele van deze medicijnafgifte apparaten bewezen een betere medicijnafgifte te bewerkstelligen dan oogdruppels.

Het doel van dit proefschrift was om een nieuwe methode voor medicijntoediening aan het oog te onderzoeken. Dit onderzoek kaderde in het Chemelot InSciTe OCDC (Ocular Coil Drug delivery and Comfort) project. In dit project werd medicijnafgifte via een oculaire coil getest. De oculaire coil is een staafje dat gemaakt is uit een gecoate, gewikkelde metaaldraad. Het midden van de coil is gevuld met kleine medicijnbolletjes (microsferen). In hoofdstuk 3 wordt verder ingegaan op het design en de mechanische eigenschappen van de oculaire coil. Er werd gekeken naar het effect van verschillende draaddiktes en diameters van de oculaire coil op zowel de flexibiliteit als de vulcapaciteit. De combinatie van beide parameters resulteerde in een flexibele oculaire coil die net genoeg vulling kon bevatten. Daarnaast is een vulmethode ontwikkeld en is kans dat microsferen uit de oculaire coil ontsnappen bestudeerd. Wanneer microsferen met een grootte van $150 \pm 10 \mu \mathrm{m}$ diameter gebruikt, konden ze niet uit de oculaire coil ontsnappen, zelfs niet wanneer de oculaire coil tot dubbel zo lang uitgetrokken werd. Om een beeld te krijgen van de 
daadwerkelijke buiging van de oculaire coil in de inferieure fornix (de binnenste holte van het onderste ooglid) werd een CT-scan gemaakt. Tegelijkertijd werden er medicijnbolletjes met ketorolac gemaakt uit polymethyl methacrylate (PMMA). Ketorolac is een niet-steroïde ontstekingsremmer die wordt gebruikt na een cataractoperatie. PMMA is een polymeer dat binnen de oogheelkunde ook gebruikt wordt in o.a. contactlenzen en intra-oculaire lenzen. Medicijnafgifte via de ketorolacgeladen microsferen in de oculaire coil was hoog tijdens eerste drie dagen, waarna de microsferen geleidelijk minder medicijn afgaven. Over een tijdsperiode van 28 dagen gaf de oculaire coil ongeveer $70 \%$ van de totale hoeveelheid geladen medicijn vrij.

In hoofdstuk 4 is gekeken naar het gedrag van de oculaire coil in proefdieren (Nieuw-Zeeland Witte konijnen). Om de farmacokinetiek te bestuderen werd de concentratie ketorolac bepaald in tranen, kamerwater en plasma. In tranen was de concentratie ketorolac hoger bij 4 uur en 24 uur in de oculaire coil groep dan in de oogdruppelgroep. Op 4 dagen, 7 dagen en 28 dagen was de concentratie ketorolac gelijk in de oculaire coil groep en de oogdruppelgroep. In kamerwater was alleen op 4 uur de concentratie ketorolac hoger in de oculaire coil groep. Op 4 dagen, 7 dagen en 28 dagen was de concentratie in de oculaire coil groep lager dan die van de oogdruppelgroep. In plasma was de concentratie ook enkel om 4 uur hoger in de oculaire coil groep dan in de oogdruppelgroep.

In de effectiviteitsstudie onderzochten we hoe goed de oculaire coil een ontstekingsreactie kan remmen. Bij aanvang van de studie werd een grote hoeveelheid (150-175 $\mu \mathrm{L}$ ) kamerwater afgenomen om een chirurgisch geïnduceerde ontstekingsreactie op te wekken. Daarna werd de ketorolac-geladen oculaire coil geplaatst, werden oogdruppels gegeven of werd er geen behandeling toegepast (controlegroep). Vervolgens is gekeken naar de totale eiwitconcentratie en de hoeveelheid ontstekingsfactoren zoals prostaglandine $\mathrm{E}_{2}\left(\mathrm{PGE}_{2}\right)$, tumor necrose factor $\alpha$ (TNF- $\alpha$ ), interleukine (IL)- 6 en IL-1 $\beta$ in tranen, kamerwater en in plasma. De $\mathrm{PGE}_{2}$ - en de IL-6-concentratie in het kamerwater lieten duidelijk zien dat er op 4 uur, 8 uur en 24 uur na het induceren van het trauma, verhoogde ontstekingswaardes meetbaar waren in de controlegroep. Met behandeling door middel van de oculaire coil en oogdruppels alleen werd een lichte verhoogde concentratie gemeten bij 24 uur. Zowel bij PGE 2 en IL-6 blokkeert het medicijn uit de oculaire coil de productie van cytokines beter dan oogdruppels. Deze resultaten tonen dat een continue afgifte van een lagere dosis medicijn uiteindelijk net zo effectief is als een intervalbehandeling met oogdruppels.

In een klinische studie werd de veiligheid en verdraagzaamheid (comfort) van een oculaire coil zonder medicijn (placebo) geëvalueerd. In deze studie zijn twee 
verschillende designs van de oculaire coil getest, een rechte en een gebogen versie. De volledige studie is uitgewerkt in hoofdstuk 5. In totaal hebben 21 proefpersoenen een rechte oculaire coil en 21 proefpersonen de gebogen coil gedragen. De retentietijd is het aantal dagen dat een proefpersoon de oculaire coil heeft gedragen tot dat de coil er spontaan uit viel of verwijderd werd. De mediaan van de retentietijd voor de rechte oculaire coil lag op 5 dagen, waarbij deze voor de gebogen oculaire coil op 12 dagen lag. Uiteindelijk hebben slechts vier proefpersonen de rechte oculaire coil voor 28 dagen in kunnen houden en zes proefpersonen de gebogen oculaire coil. Het vaakst viel de oculaire coil uit het oog tijdens oog wrijven, slapen of andere redenen. De veiligheid van de oculaire coil werd in kaart gebracht met de Efron's scoringsschaal. Deze schaal bepaalt de ernst van o.a. conjunctivale roodheid, limbale roodheid en het optreden van nieuwe bloedvaten (neovascularisatie). Tijdens het dragen van de oculaire coil werden slechts kleine verschillen in conjunctivale en limbale roodheid geobserveerd. Daarnaast was er ook geen sprake van toenemende neovascularisatie in beide groepen. Een van de ongewenste bijwerkingen van beide oculaire coils was dat deze niet op hun plaats bleven liggen, maar de neiging hadden om te migreren richting de neus. De gebogen oculaire coil migreerde tevens naar de superieure conjunctivale fornix bij drie proefpersonen. Om het comfort van beide oculaire coils te bepalen werd een vragenlijst voorgelegd aan de proefpersonen. In de vragenlijst werd o.a. gevraagd of men de coil in het oog voelt zitten en of het dragen van de coil in het oog pijnlijk, vervelend of jeukend was. De meerderheid van de proefpersonen voelden de oculaire coil niet in het oog zitten en ondervonden geen oncomfortabel gevoel. Ook vroegen we om het comfort van de oculaire coil een score te geven van 0 (erg pijnlijk) tot 100 (excellent comfort). De gemiddelde comfortscore was 88 voor de rechte coil en 93 voor de gebogen coil. Uit deze studie concludeerden we dat de oculaire coil erg veilig en comfortabel is, maar dat de huidige retentietijd te laag is om medicijnafgifte voor 28 dagen te garanderen.

Een ander onderwerp van dit proefschrift was het gebruik van Ramanspectroscopie om de concentratie medicijn (dat vrijkomt uit de oculaire coil of oogdruppels) rechtstreeks in het oog te bepalen. Ramanspectroscopie kan moleculen identificeren aan de hand van de energie in teruggekaatst licht. Hiermee is het mogelijk inzicht te krijgen in de moleculaire inhoud van een dieperliggend weefsel zonder het van buitenaf te beschadigen. In hoofdstuk $\mathbf{6}$ is gekeken is naar kwantificatie van ketorolac in het oog door middel van Ramanspectroscopie. Eerst bestudeerden we de concentratie ketorolac in varkensogen nadat ze ondergedompeld werden in een ketorolac vloeistof. Hierbij is gebruikgemaakt van drie verschillende focuslenzen: $f 60$ lens met een gonioscopie contactglas (één-spiegel), 25 x objectief (Jena lens) en een f80 lens met een cuvet houder. De volledige methodologie en kalibratie van het 
Ramansystem wordt besproken in hoofdstuk $6 \mathbf{a}$. Daarbij is tevens een MATLAB tool ontwikkeld om het Ramansignaal te zuiveren van storende achtergrondsignalen. Terwijl de f80 lens nauwkeurig de hoeveelheid medicijn in een buisje (cuvet) kon meten, konden we met deze lens geen medicijn in het oog detecteren. Daarna pasten we Ramanspectroscopie toe om ketorolac te detecteren in Nieuw-Zeeland Witte konijnen die drie keer per dag gedruppeld werden met ketorolac. Ook hier was het niet mogelijk om ketorolac in het oog te meten met de gonioscopie contactlens. Het blijkt dus dat op dit moment Ramanspectroscopie nog niet gevoelig genoeg is om medicijnen in het oog te kwantificeren. Om transparantie te geven in de behaalde resultaten van het onderzoek, is de volledige dataset gepubliceerd in hoofdstuk 6b. Dit maakt het mogelijk om in de toekomst verschillende Raman onderzoeken met elkaar te vergelijken en geeft de mogelijkheid nieuwe software te testen en te valideren.

Als laatste onderdeel van dit proefschrift is er gekeken om het graderen van oculaire roodheid objectiever te maken door middel van een 'deep-learning' software tool (hoofdstuk 7). Momenteel wordt oculaire roodheid gegradeerd op basis van een scoringskaart met referentiefoto's of getekende afbeeldingen. Deze methode is erg eenvoudig en snel, maar blijkt ook erg subjectief. Daarnaast bevat deze scoringskaart vaak slechts 4 tot 5 mogelijkheden (van 'normaal' tot 'zeer ernstig') waardoor subtiele veranderingen moeilijker te detecteren zijn. Om deze reden hebben we in deze studie de geautomatiseerde kwantificatie van oculaire roodheid onderzocht. In de eerste stap selecteerde het computerprogramma een specifiek gebied van de sclera (segmentatie). Tijdens de studie werd gebruikgemaakt van 'machine learning'. Om de software de segmentatie te leren werd een trainingdataset bestaande uit ruim 100 foto's gebruikt. Uit de resultaten bleek dat de getrainde software beter in staat was om de sclera te segmenteren dan dat onderzoekers dat manueel deden. In de tweede stap werd de roodheid daadwerkelijk berekend. Hieruit bleek dat de algoritmes van Amparo et al. en Sárándi et al. hiervoor het meest geschikt waren.

Alle resultaten uit dit proefschrift worden tot slot in hoofdstuk $\mathbf{8}$ in context geplaatst en met een kritisch oog bekeken. 


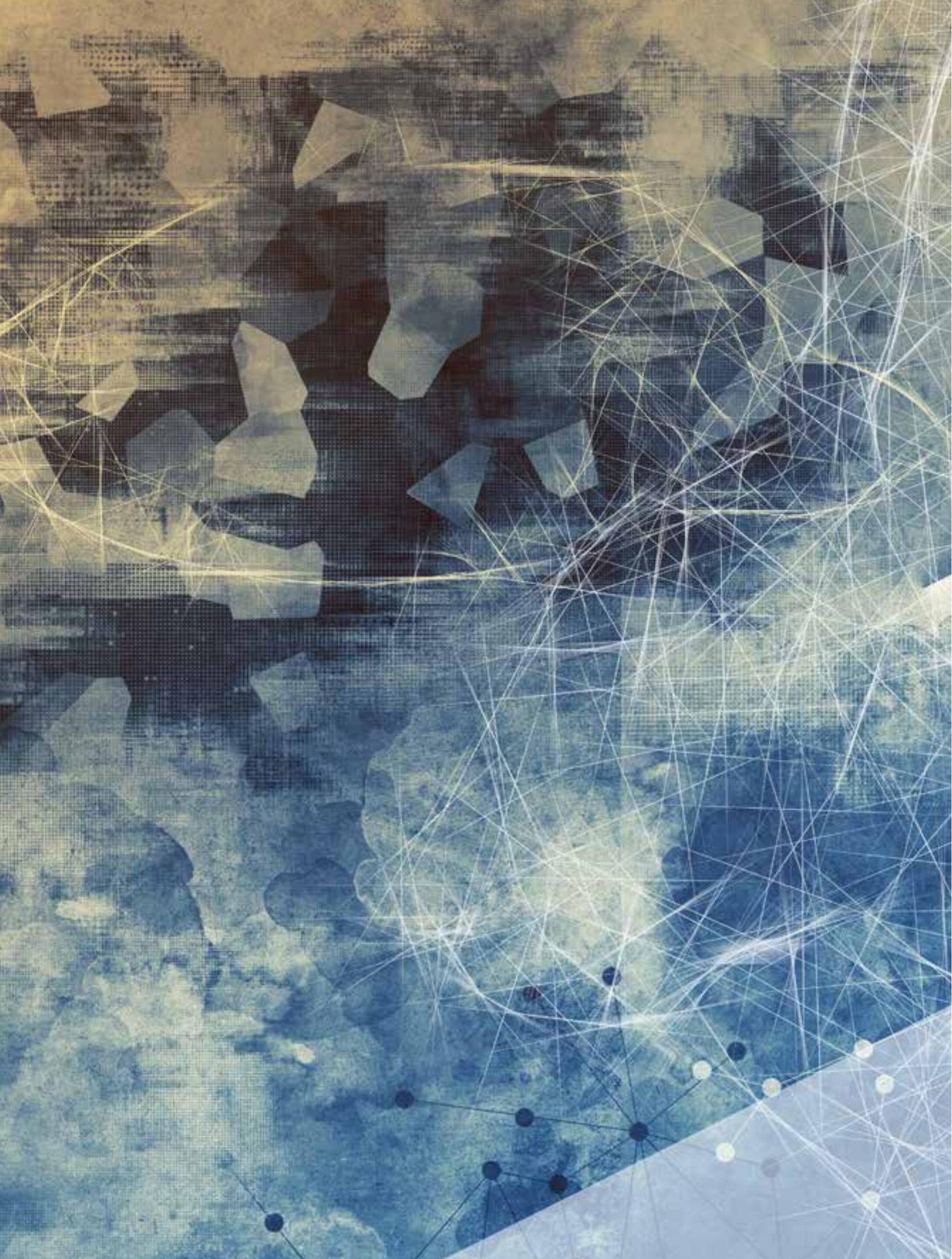




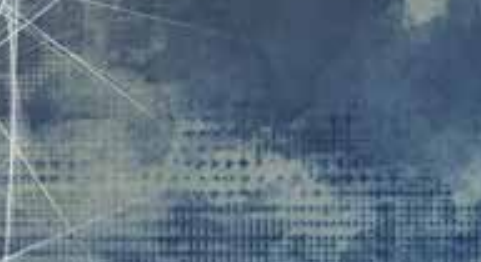

Dankwoord/.Acknowledgements

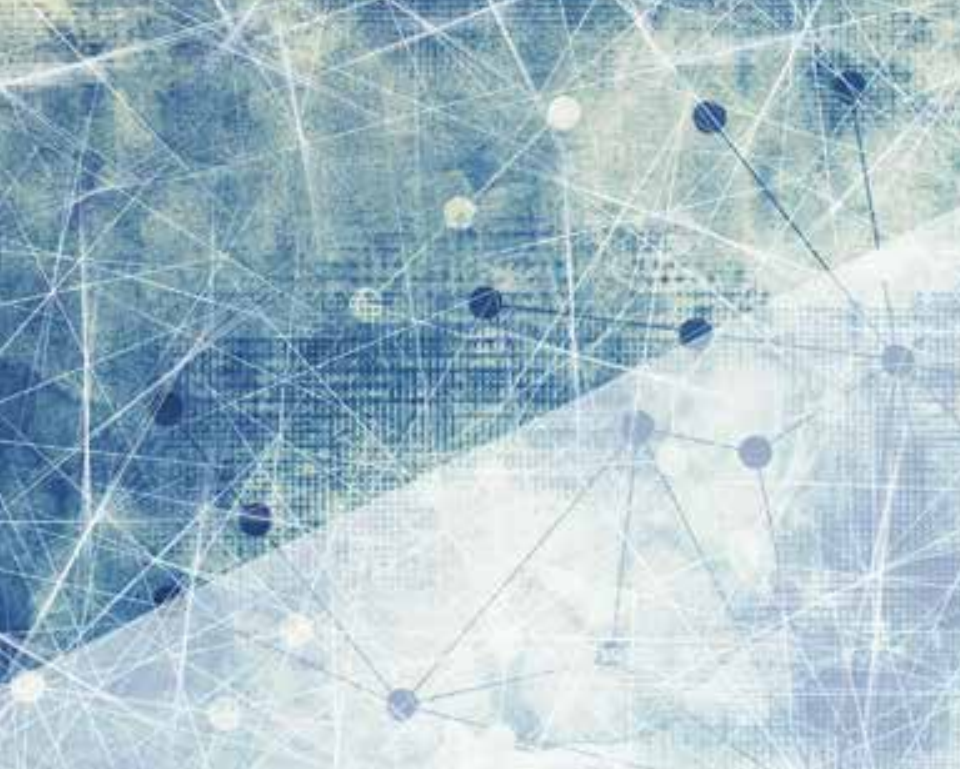

Christian J.F. Bertens 
$\mathrm{Na}$ jaren hard werken is het lastig de juiste woorden op papier te zetten om een woord van dank uit te spreken naar de mensen die mij geholpen hebben. Ik wil niemand tekort doen, maar ik wil ook zeker geen extra hoofdstuk toevoegen aan het proefschrift. Het is dan ook onmogelijk iedereen die betrokken is geweest persoonlijk te bedanken. Toch wil ik een aantal mensen bij naam noemen.

Allereerst mijn promotieteam; net als dit proefschrift heeft het promotieteam drie rotsvaste pijlers die een solide fundering vormden. Allereerst, professor Nuijts, zo heb ik je niet vaak genoemd dus zal ik nu ook maar gewoon Rudy zeggen. Ik vond het een privilege om onder jou te mogen promoveren. Je zult kopzorgen om me gehad hebben, maar door jouw strenge doch rechtvaardige toedoen hoop ik dat je op een geslaagde tijd terug kunt kijken. Het project had heel wat ups en downs maar ik durf te stellen dat ik een betere onderzoeker ben geworden door jouw hulp.

Marlies, wat hierboven staat geldt ook voor jou. Wanneer ik hulp nodig had of gewoon even wilde praten was jij er voor mij. Het kwam meer dan eens voor dat spullen aan de kant geschoven werden om het een en ander uit te leggen of om een nieuwe strategie uit te zetten. Ook voor het controleren van manuscripten, samenvattingen en presentaties heb ik veel aan je tips en opmerkingen gehad, al was het de eerste keren wel schrikken toen ik de hoeveelheid opmerkingen zag. Ik probeer in de toekomst 'CONSEQUENT' te zijn met het gebruik van woorden en kortere zinnen. Jij veel succes met je tenure track, ik heb er vertrouwen in dat dit helemaal goed gaat komen en dat ik je over enkele jaren professor Gijs mag noemen.

Frank, ook zonder jouw hulp was dit proefschrift er niet geweest. Vanaf dag één was het duidelijk dat ik voor veel bij jou terecht kon. Jouw deur stond altijd open, 'zo niet, was het klink naar beneden en duwen'. Ook in de weekenden was je bereikbaar en kon ik op je rekenen. Daarnaast kon ik altijd op je aan als er een biertje gedronken moest worden om te "netwerken".

The OCDC team, starting with Aylvin. Working with you was a mind blowing experience (in a positive way). When we had a technical meeting, we created an agenda to cover important subjects that needed to be discussed. After the second item you took over and started brainstorming on the whiteboard, driving me crazy! । came to realize that during a structured meeting you do not learn, you do not think outside the box, you do not dive into the science as much as you do when brainstorming and I sincerely miss those meetings. I hope our paths will cross again in the future and I wish you all the best with Eyegle bv. and all other projects you are working on.

All the other participants from the OCDC project, professor Tuinier, Chiara, Marty, Dilek, and Ivo. Although some collaborations were not as long as intended at the 
beginning, it was great working with you in this multidisciplinary team. Discussing one drug delivery device from all aspects: technical, biological and even commercial. I learned a lot!

The students, Sofie and Maud, I hope you were able to broaden your view and that you learned a lot during your stay at the OCDC project.

The backbone of the OCDC project and Chemelot InSciTe: Emiel, Danielle, Kurt, Filip, Odile, Yvo, Henry, Johan, Karin, Dyanne, Ingrid, Raymond, Bianca, Annabel and Kelly. The lab supervisor Chris and of course Céleste, Rowena, Youri, Jessica and Leah, thank you for all your help in the lab with the HPLC (drama machine) and with all QA/RA stuff.

I would also like to thank the support from EpFlex gmbh, especially Bernhard and Timo. Without you, the OCDC project would not have come as far as it did. It was nice to experience the workflow in your beautiful factory.

Rohit, Arkasubhra, and Swami, thank you for the hospitality and the warm welcome at the GROW research laboratory. My dearest regards, also to Tanuja and Arché for their help in the GROW lab.

I would also like to thank our collaborators from the Peter L. Reichertz Institute for Medical Informatics at the University of Braunschweig: Professor Deserno, Ekaterina, and Malte.

Binnen oogheelkunde wil ik mijn collega's ook graag bedanken. Het begon allemaal in de hoek, ellenboog tegen ellenboog met Michiel en Rob, tegenover Valentijn en Suryan. Verder nog Eline, Laura, Palwasha, Soraya, Nathalia, Ilona, Hellen, Lindsay, Lotte, Claudette, Magali, Wouter, Pascal, Ralph, Jarno, Liugi, Rana, Jurriaan, Wenting, Floortje, Marjolein en Anja. Dank ook voor de leuke borrels en gezellige etentjes met de onderzoekers. And of course not to forget, my Chinese friends, Shujin, Shuo and Shuhe. I loved eating hotpot with you and making stamppot for you. You taught me a lot of the Chinese culture; know that my door is always open for you.

Ook Theo, Tos en Roel wil ik danken voor alle uurtjes die er in het adviseren zijn gaan zitten! Fleur, dank voor de uurtjes die je geïnvesteerd hebt in het leren hechten. Veronique en Ellen, jullie bedankt voor het inplannen van alle afspraken en het rondsturen van de verzoekjes van mij.

Carroll, om de eerste vraag die je me tijdens de sollicitatie stelde alsnog te beantwoorden: de brouwmeester stopt alles in een flesje om een heerlijk bier te 
hebben, citroen of limoen is dus niet per se nodig. Maar mocht je het lekker vinden, gewoon blijven doen.

Ook alle andere medewerkers van de afdeling, heel erg bedankt voor de hulp en de fijne tijd!

Which brings me to the Neuroscience department, MHeNs. We had some good times, eating kebab during the lunches and drinking a few beers at the 'borrels'. Thanks to Glenn, Perla, Roos, Roel, Lonne, Dean, Ellis, Thomas, Martijn, Maarten, Chris, Renzo, Clara, Philippos, Sylvana, Jeroen, Jackson, Daan, Alix, Jana, Anne, Aryo, Marina, Mark, Roy, Milaine, Marion, Gusta, Artemis, Katerin, Margot, Roman, Maarten, Koen, Pim, Nick, Fred, Melinda, Sandra, Anouk and probably many, many others I forgot to list!

Jos, thanks for your honest opinion and the valuable discussions we had. In the future I hope to participate once more in the psychopharmacology course in Greece, however, now as a presenter.

Lars, Nynke en Thijs, wat hebben we goede momenten gehad met heerlijke whisky's.

Sylvia, dank voor de gezellige gesprekken en het delen van je inzichten, deze waren waardevol.

Ook de ruggengraat van het MHeNs laboratorium mag natuurlijk niet ontbreken, daarom bij deze mijn dank voor de technische ondersteuning: Hellen, Denise, Sandra, Barbie, Rachelle, Marjan en Marcella.

Voor de hulp tijdens de proefdierexperimenten ook het CPV en ondersteunend personeel heel erg bedankt: Petra, Huub, Richard, Saskia, Inger, Rachelle en Sanne.

Mijn tijd bij de Brightlands Innovation Factory is iets waar ik erg positief naar terugkijk. Leon, dank voor het accepteren van het OCDC-project, maar ook Pascal, Hugo, Nico, Kathy en Daniëlle. Ook mijn Venture Experts wil ik graag bedanken; Tom, Peter en Hans, door jullie inzichten is de entrepreneurial mindset getriggerd.

Tot slot wil ik ook nog mijn familie en vrienden bedanken. Pa en ma, bedankt voor jullie interesse in mijn proefschrift en het op de voet volgen van de totstandkoming van dit traject. Ik heb over de hele wereld gezeten tijdens mijn studies en jullie hebben mij altijd gevolgd en gesteund. Ook in tijden waarin het moeilijk was, stonden jullie voor mij klaar. Zonder jullie steun had ik dit proefschrift niet kunnen voltooien. Opa en Maria, hier is het boekje dan! Jullie hebben er vaak om gevraagd en eindelijk 
is het af. Bedankt voor al het vertrouwen in mij door de jaren heen. Het was niet altijd duidelijk waar ik mee bezig was, maar ik hoop dat nu de stukjes op hun plaats vallen. Marlissa en Yoran, Michelle en Ramon, ook jullie heel erg bedankt voor de steun tijdens dit meer-jaren-project. Huizen (ver)bouwen vergt toch meer inspanning dan aanvankelijk gedacht, maar met de hulp van familie en vrienden wordt een huis (of bouwval) toch een thuis!

Pim en Philip, na meer dan 25 jaar vriendschap nog altijd geïnteresseerd zijn in wat ik te melden heb is een wonder. Ik denk met plezier terug aan de avonden op de bank met een flesje bier en ik kijk positief naar de toekomst, waarin onze vriendschap centraal staat! Sander en Willem, ik weet dat ik meer tijd moet maken zodat we elkaar weer wat vaker kunnen zien. Als ik moest ontspannen kon ik altijd bij jullie terecht voor een goed glas bier en een barbecue, deze traditie blijven we houden! Tot slot mijn verloofde, Aline, zonder jou zou ik waarschijnlijk bezweken zijn aan de werkdruk. Jij wist op de juiste momenten mijn laptop weg te nemen en al mijn klachten over het promotietraject te weerleggen of relativeren. Achter iedere man staat een sterke vrouw, ook hier is dat helemaal waar. Ik kijk uit naar een mooie toekomst in ons fijne huisje!

Voor iedereen die ik toch nog vergeten ben:

\section{Dè ge bedankt zét dé witte!}




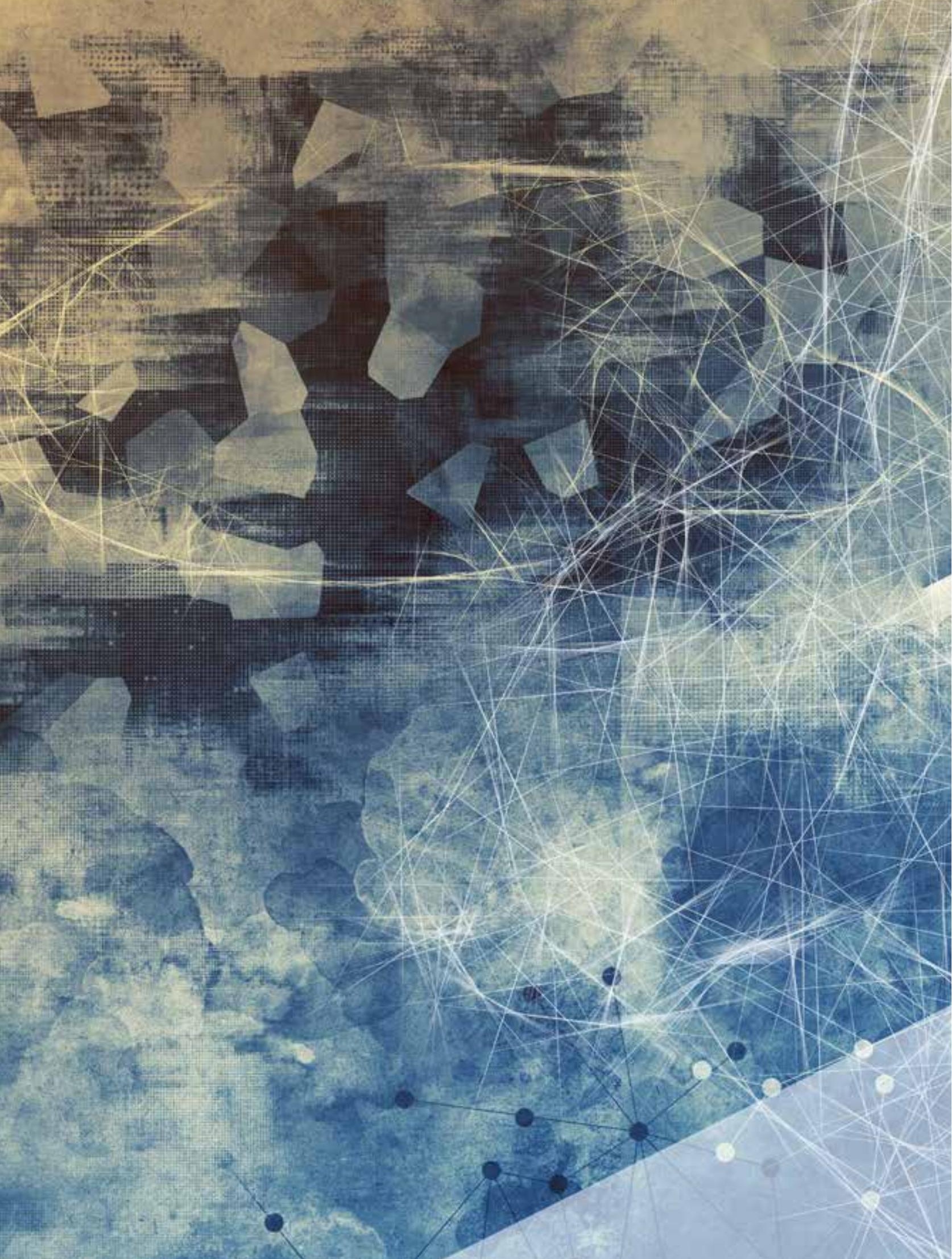


Appendix I 
Christian Johannes Fransicus Bertens was born the $4^{\text {th }}$ of April 1990 in Udenhout, the Netherlands. He graduated from senior general secondary education (HAVO) at 2 College Durendael in Oisterwijk in 2007. In 2007 he started a practical bachelor in Applied Sciences, at the Fontys University of Applied Sciences, and graduated in 2012. After graduating he decided to study Biomedical Sciences and enrolled in 2013 into the master Biomedical Sciences at Maastricht University, after obtaining a pre-master degree in Pathobiology from the Radboud University in Nijmegen. In 2014 Christian was selected for an Erasmus Mundus Joint Master Degree program. For this program he went to Toho University (Funabashi-shi, Chiba, Tokyo, JP). In 2015 he graduated from both universities in the field of Neuroscience.

In 2015 he started a PhD research project at the University Eye Clinic Maastricht under supervision of professor Rudy Nuijts. This research led to the current thesis and was a multidisciplinary collaboration between Maastricht University, Maastricht University Medical Center +, Eindhoven University of Technology, and Eyegle bv., within the framework of the Chemelot Institute for Science and Technology (InSciTe). Since January 2020 Christian is working on another project, also within the framework of InSciTe. In this project a new medical device for the treatment of glaucoma is developed in collaboration with Eindhoven University of Technology and InnFocus, Inc. a Santen Company (Miami, US). 


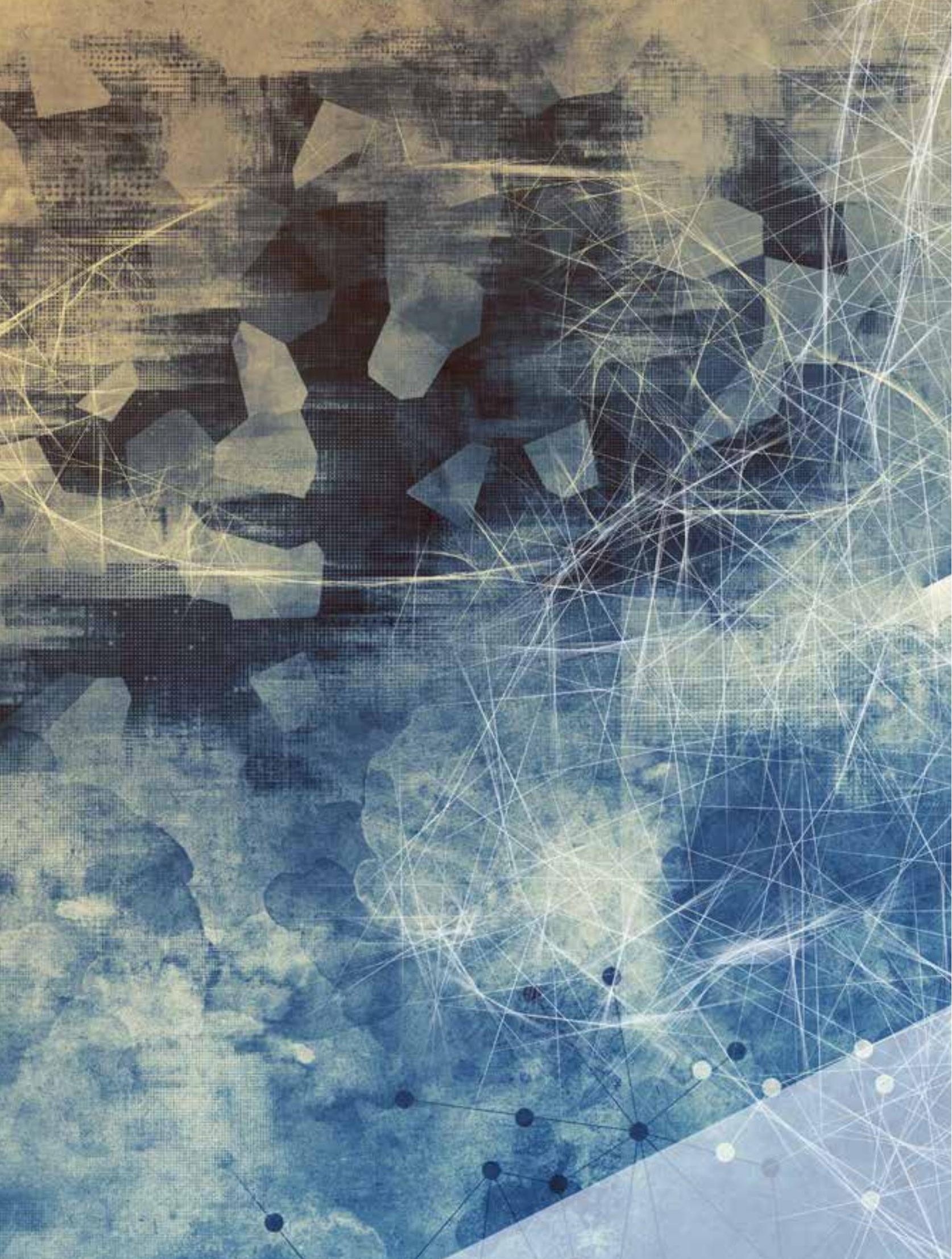




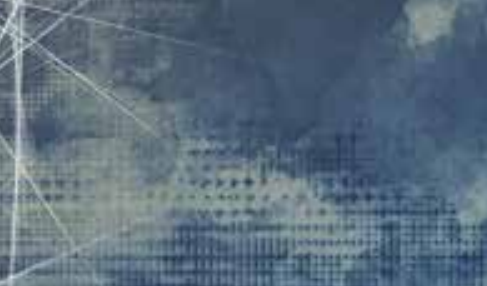

$8 \times 2$

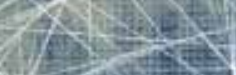

Appondix: 0 .

\section{List of publications}
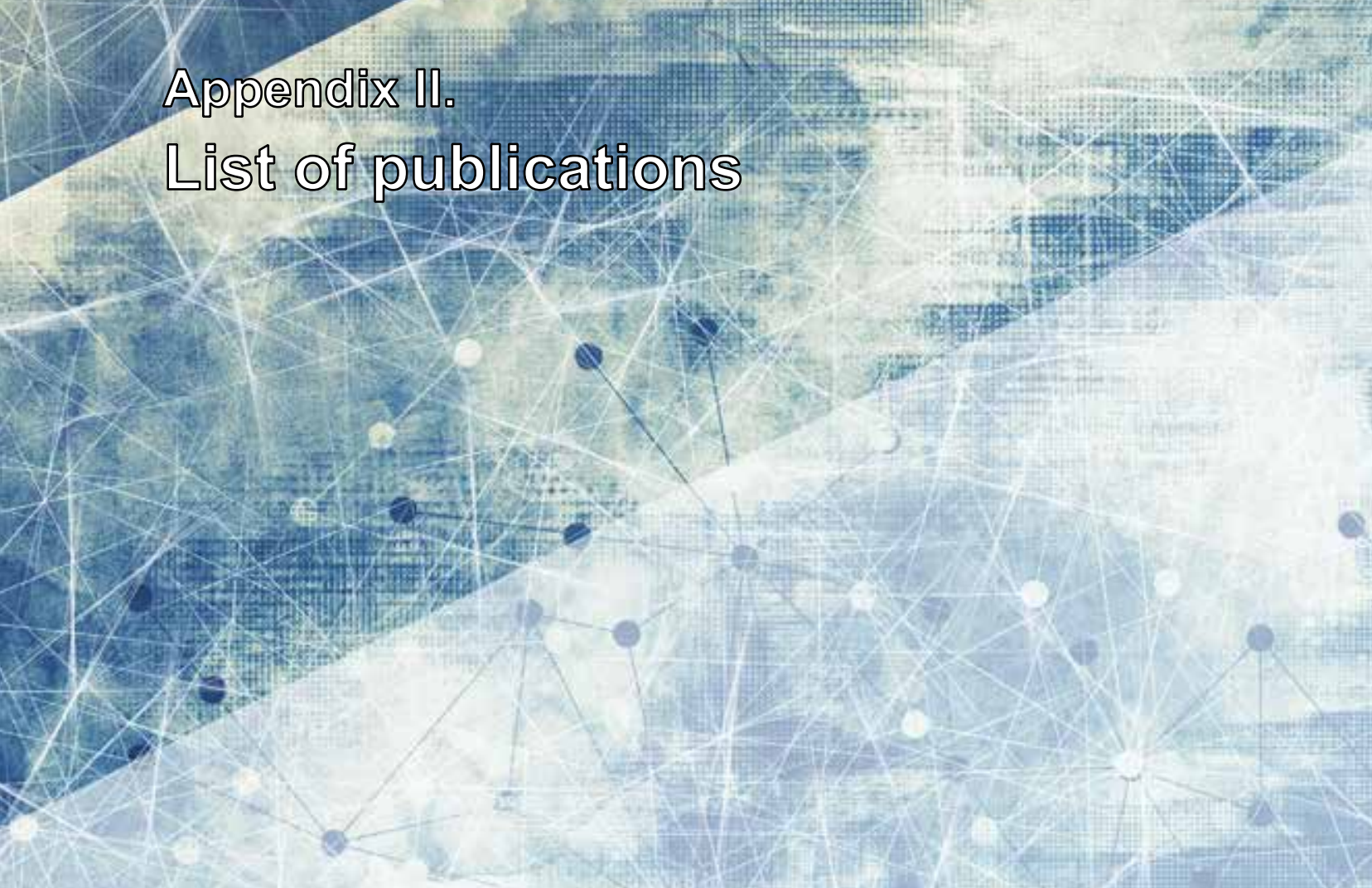


\section{Manuscripts}

2021 Bertens, C.J.F., van Mechelen, R., Berentschot, T.T.J.M., Gijs, M., Wolters, J.E.J., Gorgels, T.G.M.F., Nuijts, R.M.M.A., Beckers, H.J.M. Reproducibility of different tonometers to measure intraocular pressure: an animal study; Nature scientific reports (in preparation)

2021 Homma, H., Tanaka, H., Jin, M., Jin, X., Huang, Y., Yoshioka Y., Bertens, C.J.F., Tsumaki, K., Kondo, K., Shiwaku, H., Tagawa, K., Akatsu, H., Atsuta, N., Katsuno, M., Furukawa, K., Ishiki, A., Waragai, M., Otomo, G., Iwata, A., Yokota, T., Inoue, H., Arai, H., Sobue, G., Sone, M., Fujita, K., Okazawa, H., DNA damage in embryonic neural stem cell determines FTLDs' fate via early-stage neuronal necrosis. Science Translational Medicine (submitted May 2021)

2021 Bertens, C.J.F., Gijs, M., Dias, A.A., van den Biggelaar, F.J.H.M., Ghosh, A., Sethu, S., and Nuijts, R.M.M.A., Pharmacokinetics and efficacy of a ketorolac-loaded ocular coil in New Zealand white rabbits. Drug Delivery, 2021. 28(1): p. 400-407.

2020 Jauer, M.-L., Goel, S., Sharma, Y., Deserno, T.M., Gijs, M., Berendshot, T.T., Bertens, C.J., and Nuijts, R.M. STAPLE performance assessed on crowdsourced sclera segmentations. in Medical Imaging 2020: Imaging Informatics for Healthcare, Research, and Applications. 2020. International Society for Optics and Photonics.

2020 Bertens, C.J., Zhang, S., Erckens, R.J., van den Biggelaar, F.J., Berendschot, T.T., Webers, C.A., Nuijts, R.M., and Gijs, M., Pipeline for the removal of hardware related artifacts and background noise for Raman spectroscopy. MethodsX, 2020. 7: p. 100883.

2020 Bertens, C.J., Martino, C., van Osch, M.C., Lataster, A., Dias, A., van den Biggelaar, F.J., Tuinier, R., Nuijts, R.M., and Gijs, M., Design of the ocular coil, a new device for non-invasive drug delivery. European Journal of Pharmaceutics and Biopharmaceutics, 2020. 150: p. 120-130.

2020 Bertens, C.J., Dunker, S.L., Dias, A., van den Biggelaar, F.J., Nuijts, R.M., and Gijs, M., Safety and comfort of an innovative drug delivery device in healthy subjects. Translational vision science \& technology, 2020. 9(13): p. 35-35.

2019 Zhang, S., Bertens, C.J., Erckens, R.J., van den Biggelaar, F.J., Berendschot, T.T., Webers, C.A., Nuijts, R.M., and Gijs, M., In vitro and in vivo datasets of topically applied ketorolac tromethamine in aqueous humor using Raman spectroscopy. Data in brief, 2019. 27: p. 104694.

2019 Sirazitdinova, E., Gijs, M., Bertens, C.J., Berendschot, T.T., Nuijts, R.M., and Deserno, T.M., Validation of computerized quantification of ocular redness. Translational vision science \& technology, 2019. 8(6): p. 31-31.

2019 Bertens, C.J., Zhang, S., Erckens, R.J., van den Biggelaar, F.J., Berendschot, T.T., Webers, C.A., Nuijts, R.M., and Gijs, M., Confocal Raman spectroscopy: Evaluation of a non-invasive technique for the detection of topically applied ketorolac tromethamine in vitro and in vivo. International journal of pharmaceutics, 2019. 570: p. 118641.

2018 Bertens, C.J., Gijs, M., van den Biggelaar, F.J., and Nuijts, R.M., Topical drug delivery devices: a review. Experimental eye research, 2018. 168: $p$. 149-160. 
2014 Boross, P., van Montfoort, N., Stapels, D.A., Van Der Poel, C.E., Bertens, C., Meeldijk, J., Jansen, J.M., Verbeek, J.S., Ossendorp, F., and Wubbolts, R., FcRy-Chain ITAM Signaling Is Critically Required for Cross-Presentation of Soluble Antibody-Antigen Complexes by Dendritic Cells. The Journal of Immunology, 2014. 193(11): p. 5506-5514.

\section{Abstracts}

2020 Bertens, C., Gijs, M., Dias, A., van den Biggelaar, F., and Nuijt The ocular coil as drug delivery device, in vivo studies on safety and comfort, drug release and efficacy, ESCRS $38^{\text {th }}$ ed. Cataract Surgery: Complications \& Management; Amsterdam 2020.

2020 Gijs, M., Bertens, C., van den Biggelaar, F., Dias, A., and Nuijts, R. Safety and comfort of a non-invasive ocular drug delivery device. Acta Ophthalmologica, Volume 99, Issue S265, January 2021

2020 Bertens, C., Sirazitdinova, E., Gijs, M., Berendschot, T., Deserno, T., van den Biggelaar, F., and Nuijts, R. Validation of computerized technology to quantify ocular redness. Acta Ophthalmologica, Volume 99, Issue S265, January 2021

2019 Bertens, C., Gijs, M., van Uden, S., Dias, A., van den Biggelaar, F., and Nuijts, R.: Safety and drug-release characteristics of the ocular coil: a new non-invasive drug-delivery device to prevent intraocular inflammation after cataract surgery, ESCRS $37^{\text {th }}$ ed. Cataract Surgery: Complications \& Management; Paris 2019.

2019 Bertens, C., Gijs, M., van Uden, S., van den Biggelaar, F., and Nuijts, R. Preliminary studies of a non-invasive ocular drug delivery device. Acta Ophthalmologica, Volume 97, Issue S263, December 2019

\section{Book Chapters}

2021 Bertens, C.J.F., Gijs, M., Wolters, J.E.J., Beckers, H.J.M., Nuijts, R.M.M.A., Combination drug-device approaches in ophthalmology, Combination drug delivery system as an effective therapy against various diseases - Elsevier Science and Technology (submitted Nov 2020)

\section{Patents}

2018 Bertens, C.J.F., Gijs, M., Nuijts, R.M.M.A. APPARATUS FOR HANDLING A ROD SHAPED ELEMENT TO BE INSERTED INTO OR REMOVED FROM THE EYE OF A PATIENT (WO/2019/121173) (PCT/EP2018/084391) 
